\title{
Aircraft parameter identification for application within a fault- tolerant flight control system
}

Kerri B. Phillips

West Virginia University

Follow this and additional works at: https://researchrepository.wvu.edu/etd

\section{Recommended Citation}

Phillips, Kerri B., "Aircraft parameter identification for application within a fault-tolerant flight control system" (2011). Graduate Theses, Dissertations, and Problem Reports. 3049.

https://researchrepository.wvu.edu/etd/3049

This Dissertation is protected by copyright and/or related rights. It has been brought to you by the The Research Repository @ WVU with permission from the rights-holder(s). You are free to use this Dissertation in any way that is permitted by the copyright and related rights legislation that applies to your use. For other uses you must obtain permission from the rights-holder(s) directly, unless additional rights are indicated by a Creative Commons license in the record and/ or on the work itself. This Dissertation has been accepted for inclusion in WVU Graduate Theses, Dissertations, and Problem Reports collection by an authorized administrator of The Research Repository @ WVU.

For more information, please contact researchrepository@mail.wvu.edu. 


\title{
AIRCRAFT PARAMETER IDENTIFICATION FOR APPLICATION WITHIN A FAULT- TOLERANT FLIGHT CONTROL SYSTEM
}

\author{
by \\ Kerri B. Phillips \\ Dissertation submitted to \\ College of Engineering and Mineral Resources \\ at West Virginia University \\ in partial fulfillment of the requirements \\ for the degree of
}

Doctor of Philosophy

in

Aerospace Engineering

Approved by

Dr. Marcello Napolitano, Committee Chairperson

Dr. Bojan Cukic

Dr. Wade Huebsch

Dr. Gary Morris

Dr. Mario Perhinschi

Department of Mechanical and Aerospace Engineering

Morgantown, West Virginia

2011

Keywords: Parameter Estimation, System Identification, Aircraft, UAV, DATCOM Copyright 2011, Kerri B. Phillips 


\title{
Abstract \\ AIRCRAFT PARAMETER IDENTIFICATION FOR APPLICATION WITHIN A FAULT-TOLERANT FLIGHT \\ CONTROL SYSTEM
}

\author{
by Kerri B. Phillips
}

A parameter identification study was conducted to identify a detailed aircraft mathematical model for application within a fault-tolerant flight control system that aims to detect, identify, and accommodate for sensor and actuator failures. Specifically, a mathematical model was identified under nominal conditions for two aircraft platforms, and a model was developed for one platform under actuator failure conditions. These models are to be used in flight control law design and to account for actuator failures on the primary control surfaces for one of the research platforms. In order to accurately model the aircraft behavior following a control surface failure, the effects of an individual surface on the aircraft dynamics was estimated. Since an individual control surface deflection - for example in the event of a locked actuator - causes a coupling between the longitudinal and lateral-directional dynamics, additional terms were identified in the state space and stability and control derivative mathematical models. These models were derived from measured flight data acquired from pilot and automated computer-injected maneuvers under both nominal and failure conditions. From this analysis, the stability and control derivatives were extracted to determine the aerodynamic forces and moments on each aircraft. These aerodynamics were next introduced into a simulation environment to validate the accuracy of the identified mathematical models. A Data Compendium (DATCOM) - based analysis was conducted in order to provide a means of comparison of the models obtained through the parameter identification study and to provide constraints on parameter optimization. Finally, a confidence interval analysis was conducted to determine the reliability of the estimated values. Several simulation studies were conducted to validate the accuracy of the models for each research platform, focusing on both nominal and primary control surface failure conditions where applicable. The model outputs were compared to the measured flight data from the two respective research platforms to validate the accuracy of the estimated parameters. 


\section{Acknowledgements}

I would first like to thank my parents, Jon and Darla Phillips, for their love and support throughout my graduate work. Thank you to the rest of my family who has also supported me throughout this journey.

I would like to thank my committee chairman and research advisor, Dr. Marcello Napolitano, for his guidance and support throughout this research effort. I will be forever grateful for the opportunity to conduct research within the Flight Controls Research Lab and the education I have gained here.

I would like to acknowledge and thank my committee members, Dr. Bojan Cukic, Dr. Wade Huebsch, Dr. Gary Morris, and Dr. Mario Perhinschi for reviewing and contributing their thoughts to this research effort.

I would like to thank Dr. Giampiero Campa, who has provided me excellent guidance throughout the research project all the way from the coast of California. Thanks for sticking with me. I would also like to thank Dr. Srikanth Gururajan for his support, guidance, and mentorship. Thank you for believing in me even when I doubted myself. I would also like to extend a thank you to Dr. Yu Gu and Dr. Brad Seanor for all of their guidance with this research project.

I would like to thank the flight test members who have had a significant impact on the success of this project. Thank you to the three pilots who have flown one or both of the research platforms used in the project: Mike Eden, Mike Spencer, and Dave Ellis. Thank you to the flight testing team, past and present, who have worked diligently on construction, repairs, and instrumentation of the aircraft: Sebastian Sanchez, Jason Gross, Matt Rhudy, Zach Merceruio, Marc Gramlich, Daniele Tancredi, and Frank Barchesky. A special thanks goes to Sergio Tamayo for being a great friend and an excellent support system throughout this research effort.

I would like to thank Dr. John Kuhlman and Dr. Wade Huebsch for their support and guidance, not only during my graduate studies, but throughout my entire college career. You both truly have been role models for me. Thank you to the West Virginia Space Grant Consortium for all that they have done for me. The opportunities I had through your programs changed my life - I will never be able to say "thank you" enough. Dr. Majid Jaraiedi, Ms. Candy Cordwell, and Ms. Amy Diznoff, you all are wonderful. Please continue helping students achieve their dreams.

I would finally like to thank the West Virginia University College of Engineering and Mineral Resources and the Department of Mechanical and Aerospace Engineering for making the past 8 years the best of my life. I have learned so much throughout my studies, and I will forever be indebted to you. 
Table of Contents

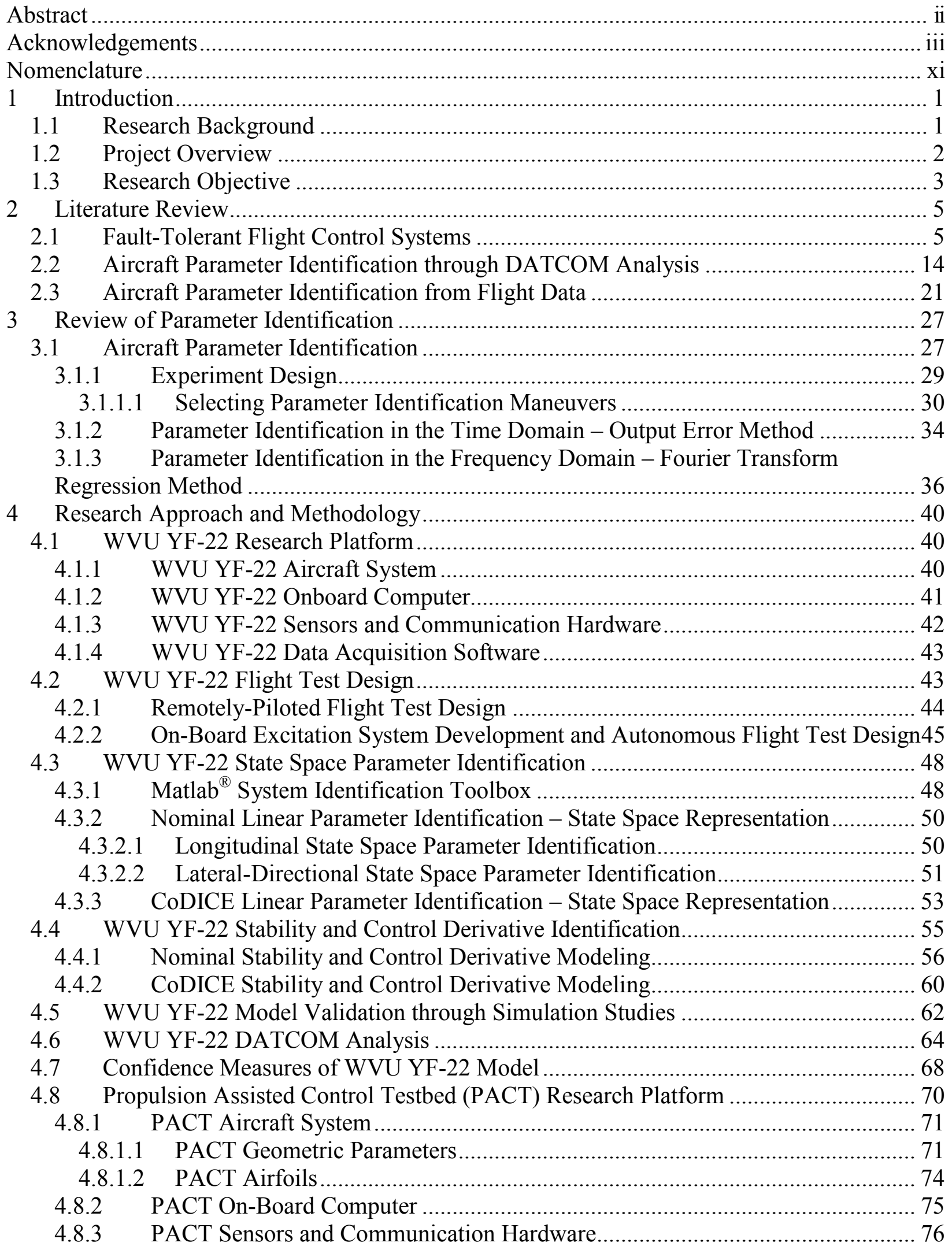




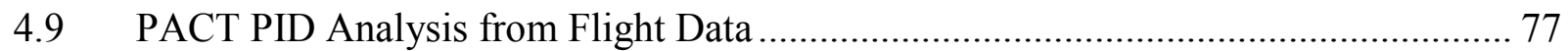

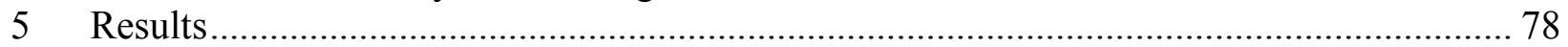

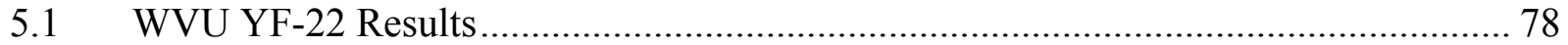

5.1.1 WVU YF-22 Linear State Space Model Identification Results............................ 78

5.1.1.1 Nominal Modeling Results ....................................................................... 78

5.1.1.2 CoDICE Modeling Results ......................................................................... 88

5.1.2 WVU YF-22 Nominal Stability and Control Derivative Identification Results ... 94

5.1.3 WVU YF-22 Application of DATCOM ........................................................ 98

5.1.4 WVU YF-22 CoDICE Stability and Control Derivative Identification Results. 103

5.1.5 WVU YF-22 Simulation Studies ................................................................ 107

5.1.6 WVU YF-22 Confidence Interval Analysis................................................ 112

5.2 WVU PACT Results ......................................................................................... 114

5.2.1 WVU PACT Linear State Space Model Identification Results ........................... 114

5.2.2 WVU PACT Stability and Control Derivative Identification........................... 120

5.2.3 WVU PACT DATCOM Analysis .................................................................. 121

5.2.4 WVU PACT Simulation Studies ............................................................ 124

5.2.5 WVU PACT Confidence Interval Analysis ................................................... 126

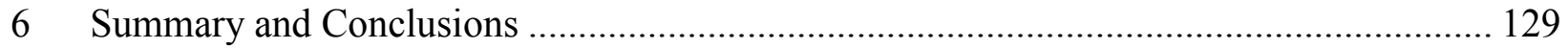

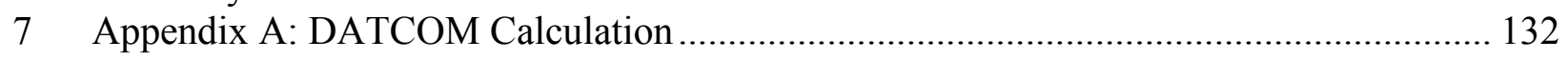

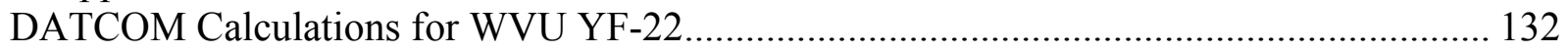

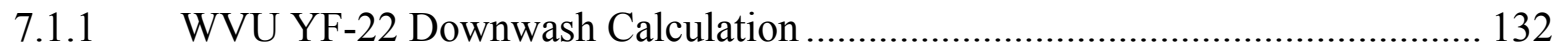

7.1.2 WVU YF-22 Stability Derivatives (DATCOM) ........................................... 134

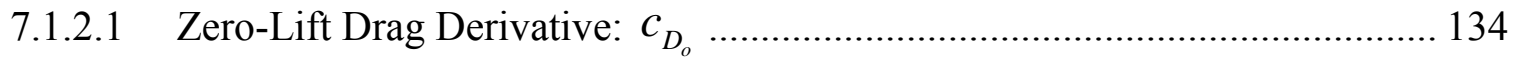

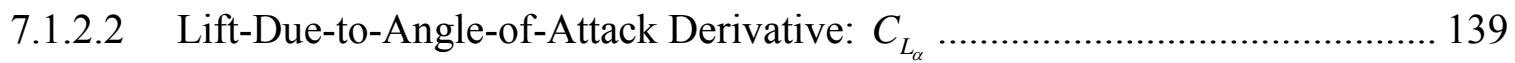

7.1.2.3 Pitching-Moment-Due-to-Angle-of-Attack Derivative: $C_{m_{\alpha}} \ldots \ldots \ldots \ldots \ldots \ldots \ldots \ldots . . . . .141$

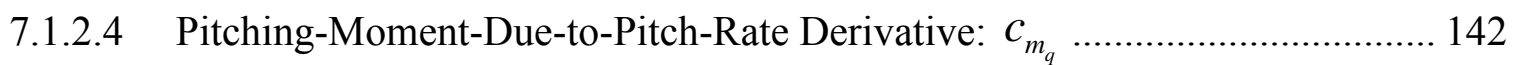

7.1.2.5 Rolling-Moment-Due-to-Sideslip Derivative: ............................................... 143

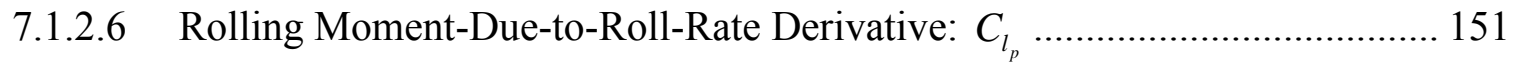

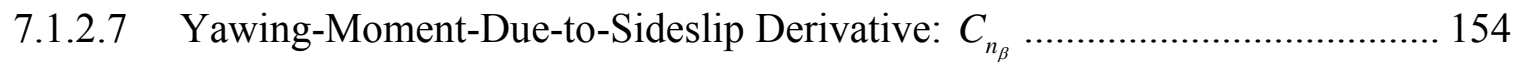

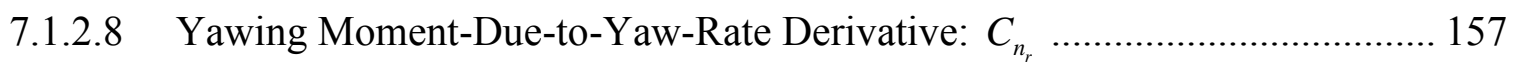

7.1.3 WVU YF-22 Control Derivatives (DATCOM) ……………………………......... 161

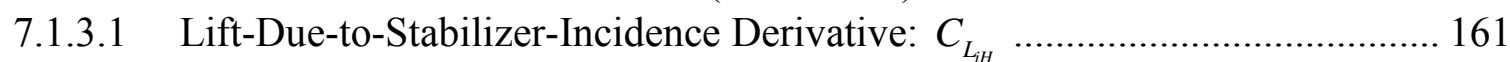

7.1.3.2 Pitching-Moment-Due-to-Stabilizer-Incidence Derivative: ${ }^{C_{m_{i H}}} \ldots \ldots \ldots \ldots \ldots \ldots \ldots . . . . . . .161$

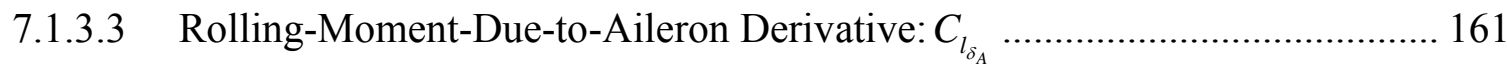

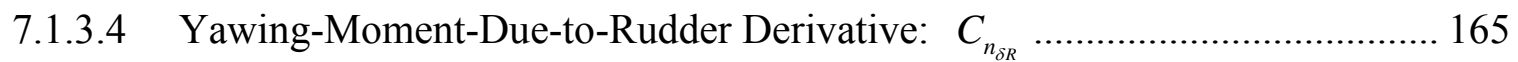

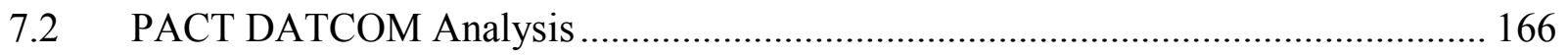

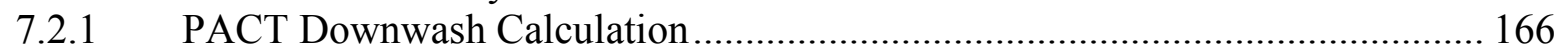

7.2.2 PACT Stability Derivatives (DATCOM) ………............................................... 168

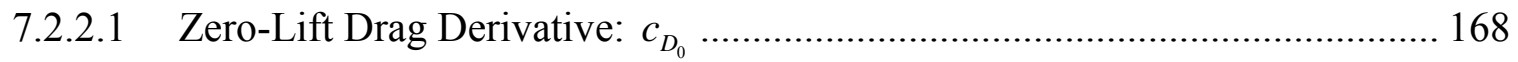

7.2.2.2 Drag-Due-to-Angle-of-Attack Derivative: ${ }^{c_{D_{\alpha}}}$.......................................... 170 


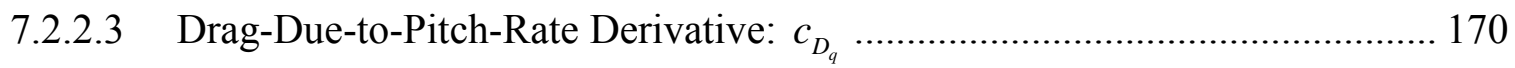

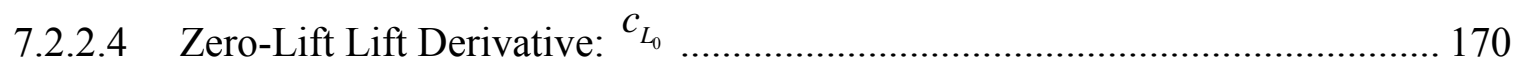

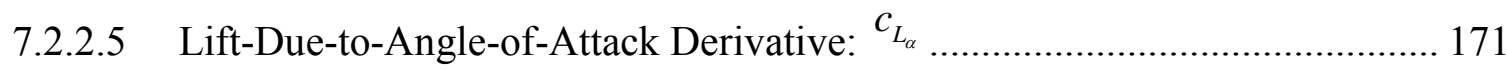

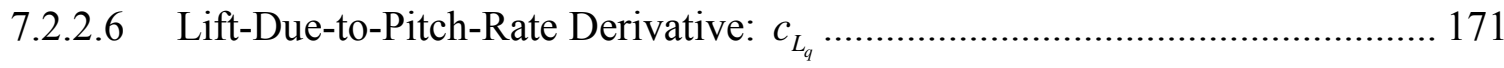

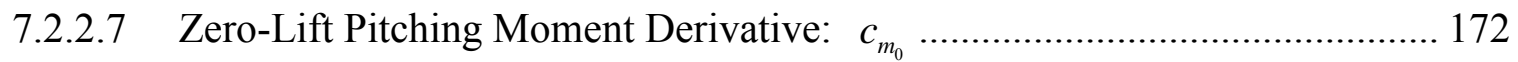

7.2.2.8 Pitching-Moment-Due-to-Angle-of-Attack Derivative: $c_{m_{\alpha}} \ldots \ldots \ldots \ldots \ldots \ldots \ldots \ldots . . . . . . . .172$

7.2.2.9 Pitching-Moment-Due-to-Pitch-Rate Derivative: $c_{m_{q}}$................................ 173

7.2.2.10 Side-Force-Due-to-Sideslip Derivative: $c_{Y_{\beta}}$.......................................... 174

7.2.2.11 Side-Force-Due-to-Roll-Rate Derivative: ${ }^{c_{Y_{p}}}$.......................................... 177

7.2.2.12 Side-Force-Due-to-Yaw-Rate Derivative: ${ }^{c_{Y_{p}}}$.......................................... 177

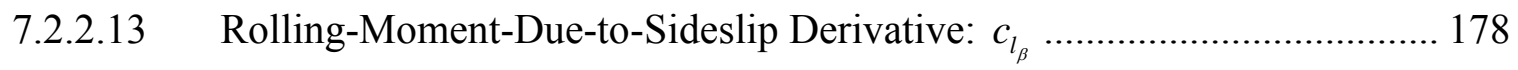

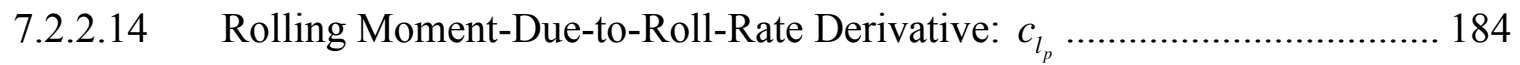

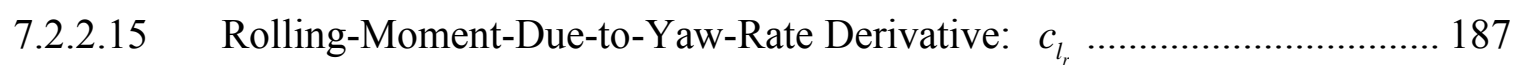

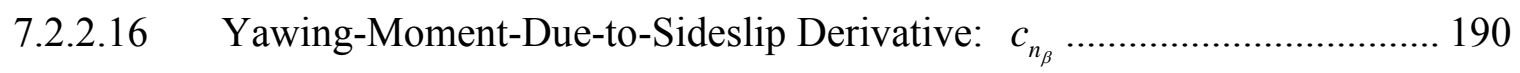

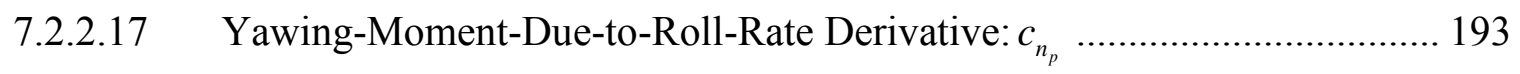

7.2.2.18 Yawing Moment-Due-to-Yaw-Rate Derivative: $c_{n_{r}}$................................ 196

7.2.3 PACT Control Derivatives (DATCOM) ………............................................ 198

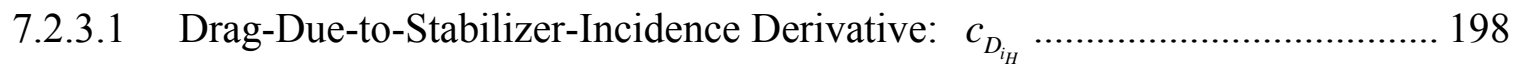

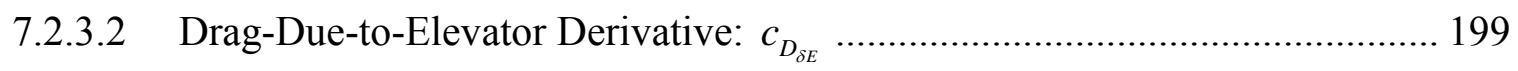

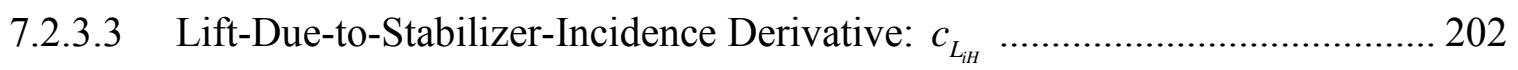

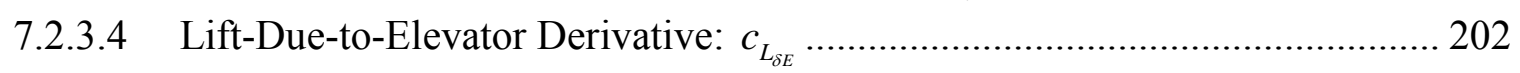

7.2.3.5 Pitching-Moment-Due-to-Stabilizer-Incidence Derivative: ${ }^{c_{i_{H}}} \quad \ldots \ldots \ldots \ldots \ldots \ldots . . . . . . .202$

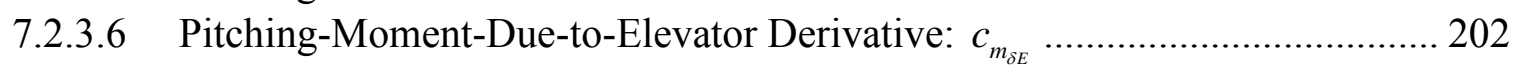

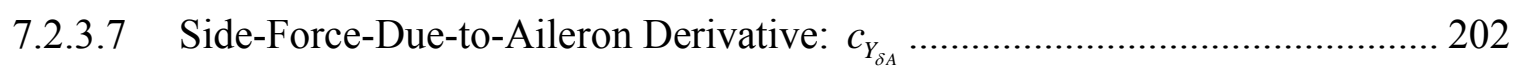

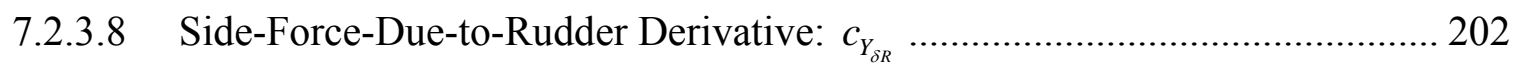

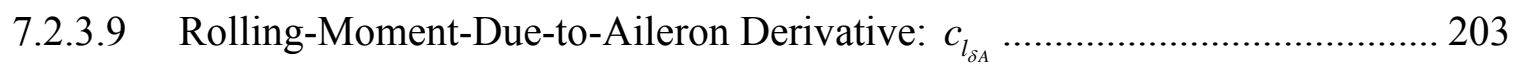

7.2.3.10 Rolling-Moment-Due-to-Rudder Derivative: $c_{l_{\delta R}}$................................... 206

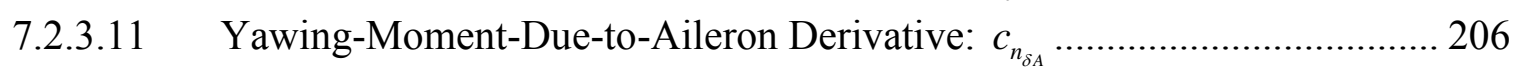

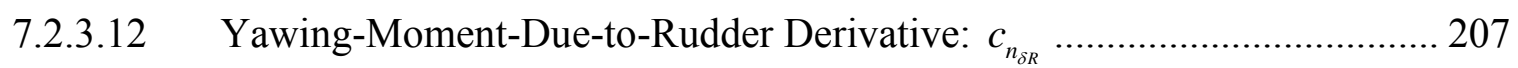

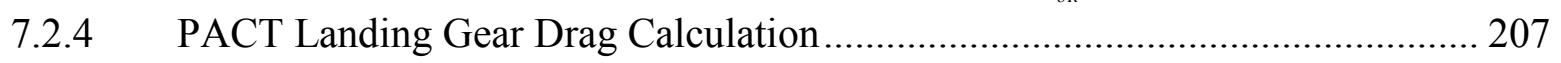

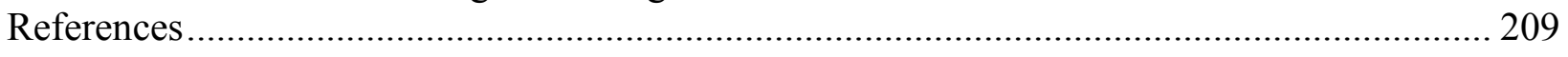




\section{List of Figures}

Figure 1: MPC Fault-Tolerant Controller [Reproduced from Reference 14] ............................... 9 Figure 2: General Block Diagram for Fault-Tolerant Flight Control System [Reproduced from

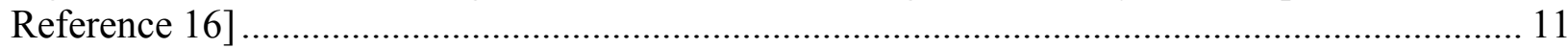

Figure 3: Self-Designing Controller Architecture [Reproduced from Reference 26] .................. 13 Figure 4: 1/5 Scale Decathlon R/C Aircraft and Digital DATCOM Representation [Reproduced from Reference 47] 16 Figure 5: Generic MAV Model for use with Missile DATCOM [Reproduced from Reference 48]

Figure 6: StablEyes Small UAV Prototype [Reproduced from Reference 50] ……………….... 18

Figure 7: Rascal 110 Representation as Input to Digital DATCOM [Reproduced from Reference

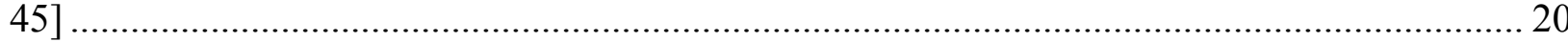

Figure 8: Rascal 110 Representation as Input to Digital DATCOM (Top View) [Reproduced

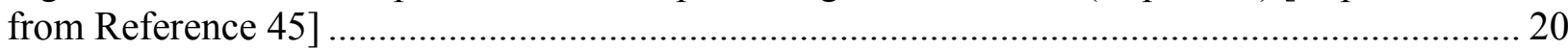

Figure 9: F-18 HARV Sideslip Derivative (Dihedral) as Function of Angle of Attack

[Reproduced from Reference 58]........................................................................................ 23

Figure 10: Dynamic System Block Diagram Representation ...................................................... 27

Figure 11: Parameter Identification Loop [Reproduced from Reference 66] ............................... 29

Figure 12: Typical Symmetric Doublet Maneuver [Reproduced from Reference ] ....................... 32

Figure 13: Output-Error Method [Reproduced from Reference 51] ........................................... 36

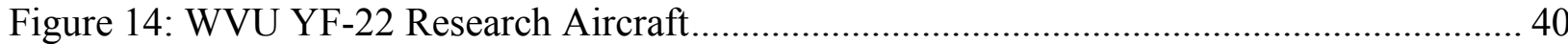

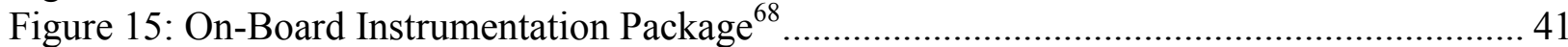

Figure 16: Virtual Leader (Blue), Simulated Follower (Red)................................................... 45

Figure 17: Projected Virtual Leader Flight Path for PID Study (in meters) ................................. 46

Figure 18: Overview of Aircraft Parameter Identification Process for WVU YF-22.................. 48

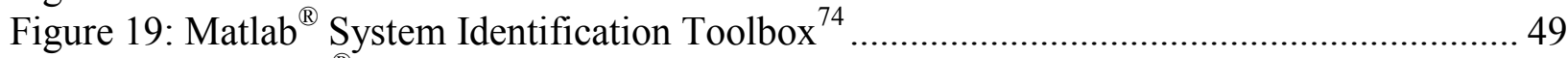

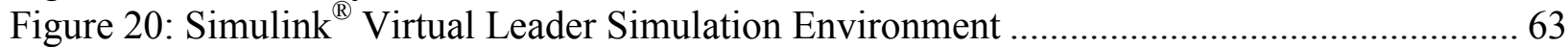

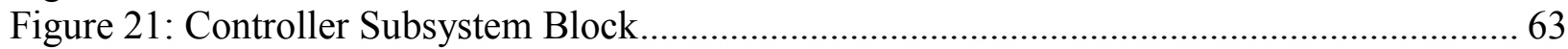

Figure 22: DATCOM Double-Delta Cranked Wing Approximation of WVU YF-22 .................. 66

Figure 23: Estimates Segregated by Input Type [Reproduced from Reference 88] ....................... 69

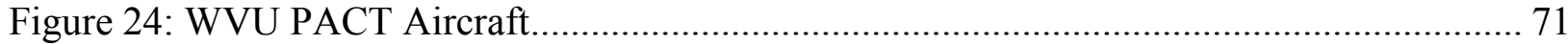

Figure 25: PACT Total Wing, Horizontal Tail, and Vertical Tail Spans .................................... 72

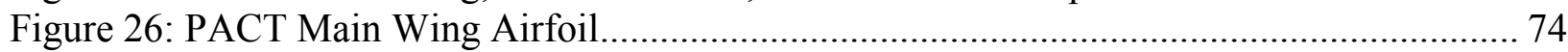

Figure 27: PACT Horizontal and Vertical Tail Airfoil............................................................. 74

Figure 28: ARIA System [Reproduced from Reference 92] ……………………................... 75

Figure 29: Flight Data Segment used for Longitudinal Linear Model Identification.................... 79

Figure 30: Flight Data Segment used for Lateral-Directional Model Identification ..................... 79

Figure 31: Virtual Leader Flight Path over WVU Jackson's Mill ............................................... 79

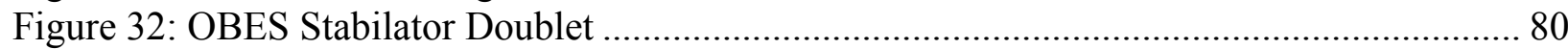

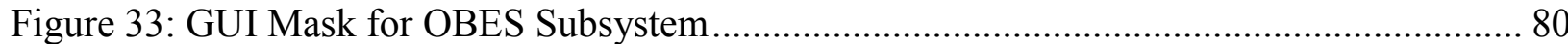

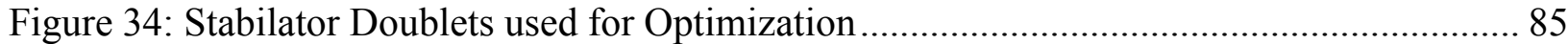

Figure 35: Rudder and Aileron Doublets used for Optimization................................................ 85

Figure 36: Measured versus Optimized Longitudinal Simulated Output ....................................... 86

Figure 37: Measured versus Optimized Lateral-Directional Simulated Output ............................ 87

Figure 38: Cross-Coupled Input Matrix Term Identification Process .......................................... 91

Figure 39: Individual Stabilator Effects on Lateral-Directional States ....................................... 92 
Figure 40: Individual Aileron Effects on Longitudinal States............................................ 92

Figure 41: Output Error Optimization of Stability and Control Derivatives ............................ 96

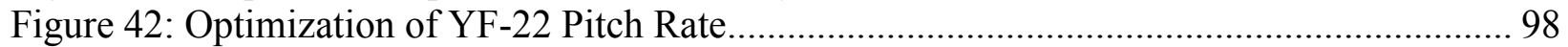

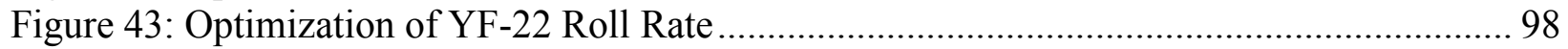

Figure 44: Optimization of Pitch Rate with 50\% DATCOM Constraints ............................... 100

Figure 45: Optimization of Roll Rate with 50\% DATCOM Constraints ................................ 100

Figure 46: Stabilator Doublet Angle of Attack ................................................................... 102

Figure 47: Stabilator Doublet Pitch Rate Response........................................................... 102

Figure 48: Rudder/Aileron Doublet Roll Rate Response .................................................. 102

Figure 49: Rudder/Aileron Doublet Yaw Rate Response.................................................. 102

Figure 50: CoDICE Stability and Control Derivative Representation.................................... 103

Figure 51: Individual Stabilator Doublet Roll Rate Response.............................................. 104

Figure 52: Individual Aileron Doublet Pitch Rate Response.................................................. 104

Figure 53: Individual Stabilator Doublet Roll Rate Response............................................... 105

Figure 54: Individual Aileron Doublet Pitch Rate Response............................................... 105

Figure 55: Stabilator Doublet Angle of Attack .................................................................. 107

Figure 56: Stabilator Doublet Pitch Rate Response.......................................................... 107

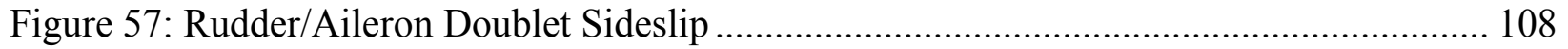

Figure 58: Rudder/Aileron Doublet Yaw Rate .................................................................. 108

Figure 59: Rudder/Aileron Doublet Roll Rate................................................................. 108

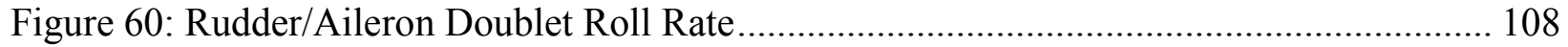

Figure 61: Rudder/Aileron Doublet Sideslip .................................................................... 109

Figure 62: Rudder/Aileron Doublet Yaw Rate ................................................................. 109

Figure 63: Pitch and Roll Rate Response to Single OBES Stabilator Doublet ........................ 109

Figure 64: Pitch and Roll Rate Response to Single Manual Stabilator Doublet ...................... 109

Figure 65: Pitch and Roll Rate Response to Single OBES Aileron Doublet........................... 110

Figure 66: Pitch and Roll Rate Response to Single Manual Aileron Doublet.......................... 110

Figure 67: Angle of Attack Response to Single Manual Aileron Doublet ............................... 110

Figure 68: Pitch and Roll Rate Response during Highly Banked Turns ................................ 110

Figure 69: Simulation Results for Virtual Leader Tracking with OBES Maneuvers ................ 112

Figure 70: Flight Data Segment used for Longitudinal Linear Model Identification................. 115

Figure 71: Flight Data Segment used for Lateral-Directional Model Identification ................. 115

Figure 72: Measured Elevator Deflections used for Optimization ........................................ 119

Figure 73: Measured Rudder and Aileron (Right) Deflections used for Optimization ............. 119

Figure 74: Optimization Results for Angle of Attack and Pitch Rate ................................... 119

Figure 75: Optimization Results for Sideslip, Roll Rate, and Yaw Rate............................... 119

Figure 76: SolidWorks ${ }^{\circledR}$ CAD Drawing of PACT Aircraft .................................................. 120

Figure 77: PACT Optimization Result for Angle of Attack during Elevator Doublet .............. 123

Figure 78: PACT Optimization Result for Pitch Rate during Elevator Doublet ...................... 123

Figure 79: PACT Optimization Result for Roll Rate during Aileron and Rudder Doublets ...... 124

Figure 80: PACT Optimization Result for Yaw Rate during Aileron and Rudder Doublets ..... 124

Figure 81: Simulation Results for Angle of Attack with Elevator Doublet............................ 125

Figure 82: Simulation Results for Pitch Rate with Elevator Doublet ................................... 125

Figure 83: Simulation Results for Roll Rate with Elevator Doublet .................................... 125

Figure 84: Simulation Results for Yaw Rate with Aileron Doublet .................................... 125

Figure 85: Factor $K_{A}$ used for Calculating Downwash [Reproduced from Reference 42] ........ 132 
Figure 86: Factor $K_{\lambda}$ used for Calculating Downwash [Reproduced from Reference 42]......... 133

Figure 87: Factor $K_{H}$ used for Calculating Downwash [Reproduced from Reference 42] ........ 133

Figure 88: Spanwise (Left) and Longitudinal (Right) Variations in Dynamic Pressure Ratio

[Reproduced from Reference 42].

Figure 89: Lifting-Surface Correlation Factor for Subsonic Minimum Drag [Reproduced from

Reference 41].... 136

Figure 90: Wing-Body Interference Correlation Factor - Subsonic Speeds [Reproduced from

Reference 41].... 137

Figure 91: Body Cross-Section Geometries [Reproduced from Reference41] ........................ 138

Figure 92: Wing Lift-Curve Slope Correction Factor [Reproduced from Reference 41].......... 139

Figure 93: Lift Ratios KW(B) and KB(W) - Slender Body Theory - Fixed Incidence - All

Speeds [Reproduced from Reference 41] ............................................................................. 140

Figure 94: Aircraft Geometric Parameters [Reproduced from Reference 41] .......................... 142

Figure 95: Fuselage Longitudinal Geometric Parameters [Reproduced from Reference 41] .... 143

Figure 96: Wing Sweep Contribution to $C_{l_{\beta}}$ [Reproduced from Reference 41] ...................... 145

Figure 97: Compressibility Correction Factor to Sweep Contribution to Wing $C_{l_{\beta}}$ [Reproduced

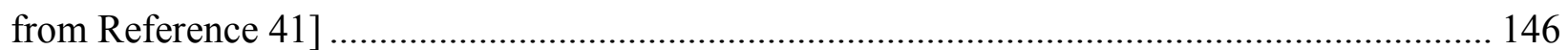

Figure 98: Fuselage Correction Factor [Reproduced from Reference 41] ................................ 147

Figure 99: Aspect Ratio Contribution to Wing $C_{l_{\beta}}$ [Reproduced from Reference 41] .............. 147

Figure 100: Charts for Estimating the Sideslip Derivative $\left(\Delta C_{Y_{\beta}}\right)_{V(W B H)}$ for Twin Vertical Tails

[Reproduced from Reference 41] ................................................................................. 150

Figure 101: Roll-Damping Parameter at Zero Lift $(\lambda=0.25)$ [Reproduced from Reference 41]

Figure 102: Drag-Due-To-Lift Roll-Damping Parameter [Reproduced from Reference 41]..... 153

Figure 103: Empirical Factor $\mathrm{K}_{\mathrm{N}}$ Related to Sideslip Derivative $C_{n_{\beta}}$ for Body + Wing-Body

Interference [Reproduced from Reference 41] .................................................................. 155

Figure 104: Effect of Fuselage Reynolds number on Wing-Body $C_{n_{\beta}}$ [Reproduced from

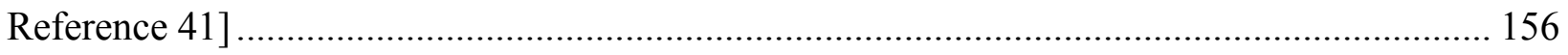

Figure 105: Low-Speed Drag-Due-To-Lift Yaw-Damping Parameter [Reproduced from

Reference 41] ......................................................................................................... 159

Figure 106: Low-Speed Profile-Drag Yaw-Damping Parameter [Reproduced from Reference 41]

Figure 107: Basic Layout used for Estimating $K_{b}$ [Reproduced from Reference 41] ............... 162

Figure 108: Aileron Rolling Moment Parameter for $\lambda=0.5$ [Reproduced from Reference 41] 163

Figure 109: Correction Factor for Plain Flap or Aileron Lift [Reproduced from Reference 41] 164

Figure 110: Lift Effectiveness of a Plain Flap or Aileron [Reproduced from Reference 41] ... 165

Figure 111: Factors for Computing Downwash [Reproduced from Reference 42].................. 167

Figure 112: Wing-Fuselage Interference Correlation Factor [Reproduced from Reference 41] 168

Figure 113: Lifting Surface Correlation Factor for Subsonic Minimum Drag [Reproduced from

Reference 41] ........................................................................................................... 169

Figure 114: Turbulent Mean Skin-Friction Coefficient of Insulated Flat Plate [Reproduced from

Reference 41] ................................................................................................................. 169

Figure 115: Definition of Required PACT Geometric Parameters........................................ 172 
Figure 116: Correction Constant for Wing Contribution to Pitch Damping [Reproduced from

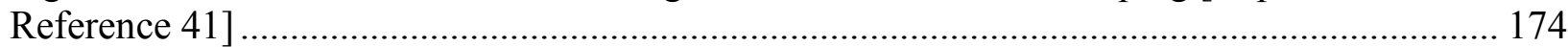

Figure 117: Wing-Body Interference Factor [Reproduced from Reference 42]....................... 175 Figure 118: Empirical Factor for Estimating Side-Force due to Sideslip of a Single Vertical Tail

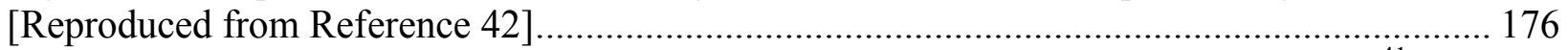

Figure 119: Definition of Wing-Fuselage Parameters for Low and High Wing Aircraft ${ }^{41}$........ 177

Figure 120: PACT Geometry for Locating Vertical Tail........................................................ 177

Figure 121: Wing Sweep Contribution to Rolling Moment due to Sideslip [Reproduced from

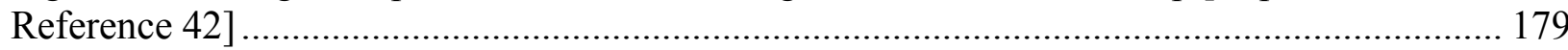

Figure 122: Compressibility Correction Factor to Wing Sweep [Reproduced from Reference 42]

Figure 123: Fuselage and Wing Geometric Parameters ......................................................... 181

Figure 124: Fuselage Correction Factor [Reproduced from Reference 42] .............................. 182

Figure 125: Aspect Ratio Contribution to Rolling Moment due to Sideslip [Reproduced from

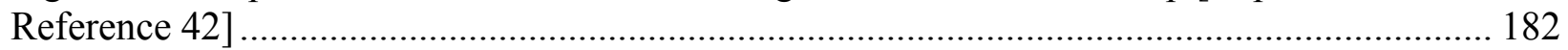

Figure 126: Wing Geometric Dihedral Contribution to Rolling Moment due to Sideslip

[Reproduced from Reference 42].................................................................................... 183

Figure 127: Compressibility Correction to Wing Dihedral Effect [Reproduced from Reference

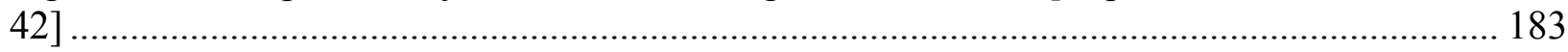

Figure 128: Effect of Wing Twist on Rolling Moment due to Sideslip [Reproduced from

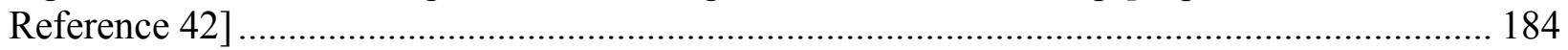

Figure 129: Roll Damping Parameter at Zero Lift [Reproduced from Reference 42]................ 185

Figure 130: Drag-due-to-Lift Roll-Damping Parameter [Reproduced from Reference 42] ....... 186

Figure 131: Wing Rolling Moment due to Yaw Rate Derivative (Lifting Effect) [Reproduced

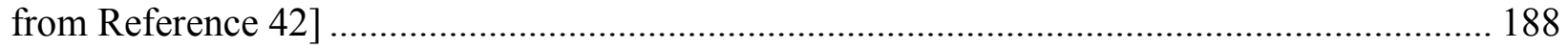

Figure 132: Effect of Wing Twist [Reproduced from Reference 42] ........................................ 189

Figure 133: Effect of Symmetric Flap Deflection on $c_{l_{r}}$ [Reproduced from Reference 42] ....... 189

Figure 134: Empirical Factor $K_{N}$ Accounting for Wing-Body Interference with Directional

Stability [Reproduced from Reference 42] ...

Figure 135: Effect of Fuselage Reynold's Number on Wing-Body Directional Stability

[Reproduced from Reference 42].

Figure 136: Effect of Wing Twist on Yawing-Moment-due-to-Roll-Rate Derivative

[Reproduced from Reference 42].

Figure 137: Effect of Symmetrical Flap Deflection on Yawing-Moment-due-to-Roll-Rate

Derivative [Reproduced from Reference 42]

Figure 138: Low-Speed Drag-due-to-Lift Yaw-Damping Parameter [Reproduced from Reference

42]

Figure 139: Low-Speed Profile Drag Yaw-Damping Parameter [Reproduced from Reference 42]

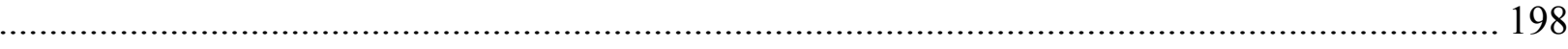

Figure 140: Correction Factor for Plain Flap Lift [Reproduced from Reference 42] ................. 200

Figure 141: Lift Effectiveness of a Plain Flap [Reproduced from Reference 42] ..................... 200

Figure 142: Correction Factor for Nonlinear Lift Behavior of Plain Flaps [Reproduced from

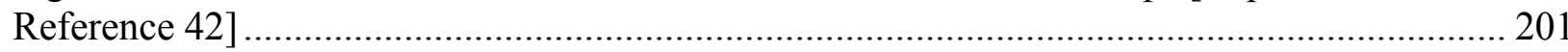

Figure 143: Aspect Ratio and Flap-Chord Ratio Effect on Three-Dimensional Flap Effectiveness

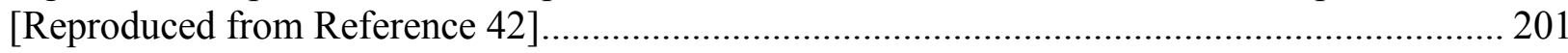


Figure 144: Effect of Symmetrical Flap Deflection on Yawing-Moment-due-to-Roll-Rate

Derivative [Reproduced from Reference 42] .................................................................. 204

Figure 145: Correction Factor for Plain Flap Lift [Reproduced from Reference 42] ................ 205

Figure 146: Lift Effectiveness of a Plain Flap [Reproduced from Reference 42] ................... 206

Figure 147: Correlation Constant for Yawing Moment due to Aileron Deflection [Reproduced

from Reference 42] ............................................................................................. 207

Figure 148: Nose Gear Geometric Parameters Necessary to Calculate the Drag Coefficient.... 208

\section{Nomenclature}

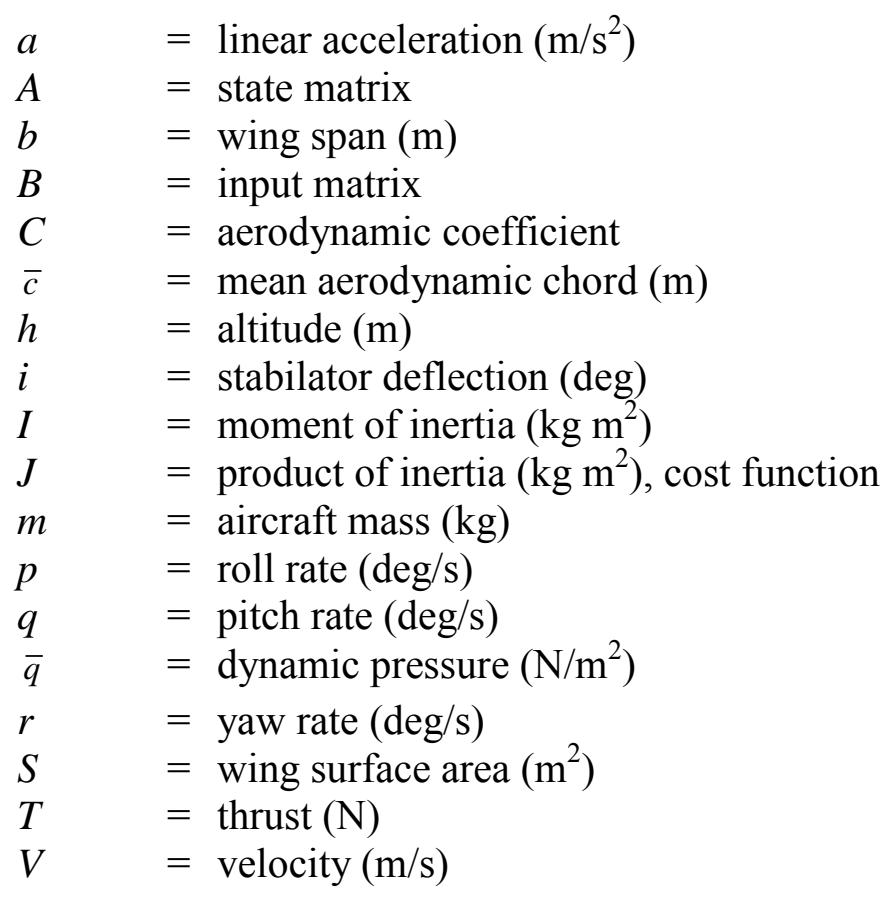

\section{Greek Letters}

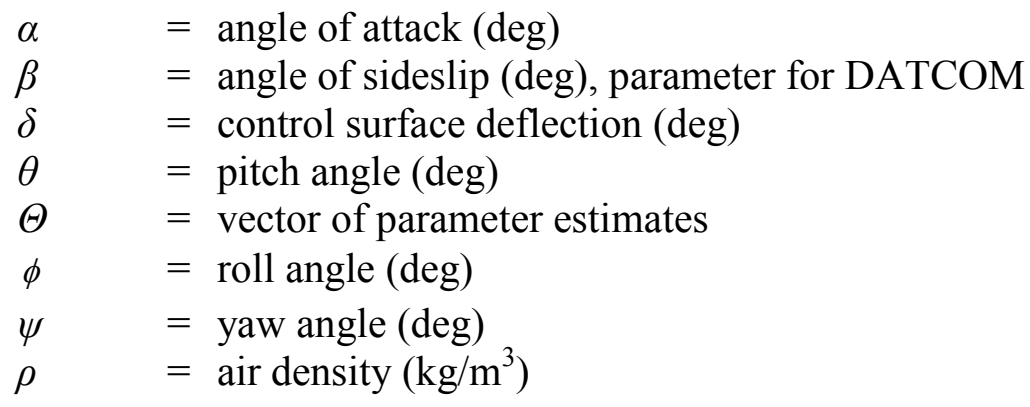

\section{Subscripts}

$\begin{array}{ll}a & =\text { aileron } \\ D & =\text { drag } \\ e & =\text { elevator } \\ H & =\text { stabilator } \\ l & =\text { rolling moment } \\ L & =\text { lift, left }\end{array}$




$\begin{array}{ll}m & =\text { pitching moment } \\ n & =\text { yawing moment } \\ r & =\text { rudder } \\ R & =\text { right } \\ x x & =\text { about the } \mathrm{x} \text {-axis (body) } \\ x z & =\text { about the } \mathrm{x} \text { and } \mathrm{z} \text { axes (body) } \\ Y & =\text { side force } \\ y y & =\text { about the } \mathrm{y} \text {-axis (body) } \\ z z & =\text { about the } \mathrm{z} \text {-axis (body) }\end{array}$

$$
\begin{aligned}
& \text { Acronyms } \\
& A L G=\text { Longitudinal state matrix } \\
& A L T=\text { Lateral-directional state matrix } \\
& B L G=\text { Longitudinal input matrix } \\
& B L T=\text { Lateral-directional input matrix } \\
& E C U=\text { Engine control unit } \\
& F C S=\text { Flight control system } \\
& F T R=\text { Fourier Transform Regression } \\
& G U I=\text { Graphical user interface } \\
& I M U=\text { Inertial measurement unit } \\
& N R L S=\text { Normalized Recursive Least Squares } \\
& O B C=\text { Onboard computer } \\
& O B E S=\text { Onboard excitation system } \\
& P C B=\text { Printed Circuit Board } \\
& P D F=\text { Power density function } \\
& P I D=\text { Parameter identification } \\
& P W M=\text { Pulse width modulation } \\
& R T A I=\text { Real Time Application Interface } \\
& R T W=\text { Real-Time Workshop } \\
& U A V=\text { Unmanned Aerial Vehicle } \\
& V L=\text { Virtual leader } \\
& W V U=\text { West Virginia University }
\end{aligned}
$$




\section{Introduction}

\subsection{Research Background}

The use of unmanned aircraft for validation and verification of flight control laws has become an appealing option among researchers due to the high cost and risks associated with flight testing programs of manned aircraft. Unmanned aerial vehicles (UAVs) have been extensively used for the development of nonlinear control schemes for "conventional" aircraft guidance, navigation, and control systems. However, the use of UAVs is even more appropriate for the evaluation of control schemes for non-nominal conditions, such as those associated with failures to components of the flight control system.

The failure of primary control surfaces has been recognized as one of the main causes of accidents in military and civilian aviation, contributing to $25 \%$ of commercial aircraft accidents in the past 60 years ${ }^{1}$. Both military and civilian transport aircraft are required to have control surface actuator redundancy, including back-up control authority and redundancy in power supplies. Despite these redundancies accidents involving primary control surface failures have occurred, indicating the need for fault-tolerant flight control system development. Examples of accidents involving primary control surface failures include USAir Flight 427 and United Airlines Flight 585, both caused by a faulty servo valve locking the rudder at its blowdown $\operatorname{limit}^{2,3}$, and United Airlines Flight 232, which had a catastrophic right engine failure causing the debris to rupture the hydraulic lines associated with the right elevator ${ }^{4}$. In the case of USAir Flight 427, the locked rudder caused the Boeing 737 to crash within 28 seconds of the failure from an altitude of 5,000 $\mathrm{ft}$. Given the limited altitude and the low airspeed, the pilots had virtually no time for detecting and isolating the failure and, therefore, for making any attempt for a recovery maneuver. During the accident investigation, Boeing test pilots involved in both flight and simulator testing revealed that a "successful recovery required immediate flight crew recognition of the upset event and subsequent prompt control wheel inputs to the full authority of the airplane's roll control limits and pitch flight control inputs to maintain a speed above the crossover airspeed"2. An interesting point is that during the accident investigation for Flight 427 , investigators noted a crucial similarity between the reaction of the pilots on Flight 585 (March 1991) and Flight 427 (September 1994): neither pair of pilots had any clue as to the cause of the upset event ${ }^{5}$. 
With $31 \%$ of all commercial aircraft accidents in the past decade being attributed to some type of mechanical failure ${ }^{5}$, the need for fault-tolerant flight control systems is evident. In providing such an application the development of an improved mathematical model through a more comprehensive modeling effort is required for improved understanding of the aircraft dynamics at post failure conditions.

In providing such an application, the development of an improved mathematical model through a more comprehensive modeling effort is required for improved understanding of the aircraft dynamics during post failure conditions. Specifically, a failure involving a locked actuator does not affect the aerodynamic characteristics of the control surface; however, under failure conditions the aircraft mathematical model must include the contribution of each left and right surface ${ }^{6}$, since individual control surface deflections affect both the longitudinal and lateraldirectional dynamic responses of the aircraft. For example, individual left or right stabilator excitation effects must be included in the determination of the lateral-directional aerodynamic derivatives since roll and yaw responses will develop, in addition to a pitching moment following a stabilator failure. As a result, additional stability and control derivatives are introduced based on the modeling of the individual left and right control surface inputs. The coupling of the longitudinal and lateral-directional dynamics can be represented by separating the corresponding terms in the aerodynamic modeling equations into left and right control surface components and including their individual effects. Thus, the deflections of each individual control surface must be accounted for in the modeling of the longitudinal and lateraldirectional aerodynamic forces and moments.

\subsection{Project Overview}

The Flight Control Research Lab (FCRL) at WVU was awarded a three year NASA EPSCoR Grant to develop and test a fault-tolerant flight control system to handle both sensor and actuator failures. Within this effort, actuator and sensor failure detection, identification, and accommodation (AFDIA/SFDIA) schemes will be developed to maintain safe flight for an unmanned aerial vehicle platform under specific failure scenarios. A portion of this effort will focus on accommodating for sensor failures with the development of a new avionics platform and sensor fusion algorithms.

For the AFDIA scheme development, an accurate linear mathematical model of the aircraft is required. Additionally, the stability and control derivatives representing the aircraft is necessary 
for simulation studies prior to flight testing of the new control laws. A detailed modeling effort was conducted using experimental flight data for parameter estimation. Flight tests were designed to incorporate nominal flight as well as flight with actuator failures (simulating locked control surfaces), so the dynamic behavior of the aircraft could be modeled under these conditions. Simulation studies were conducted to validate the identified models, and USAF DATCOM was utilized as a tool for comparison and optimization. A confidence analysis was implemented to provide a measure of reliability of the estimated parameters of the linear state space model used for control law design.

Once the aircraft dynamics are modeled under actuator failure conditions and validated through simulation studies, the linear mathematical model will be used in the development of a set of fault-tolerant flight control laws. Specifically, a Linear Quadratic Tracking controller will be designed to obtain feedback gains for an inner-loop controller using the derived nominal aircraft model. An adaptive algorithm will be developed using the linear model derived under actuator failure conditions to handle control surface failures in flight using other available surfaces and differential thrust.

\subsection{Research Objective}

The objective of this research was to identify models of the WVU YF-22 and Propulsion Assisted Control Test bed (PACT) aircraft from flight data that accurately represent their dynamic behavior under nominal conditions and following a primary control surface failure for application within a fault-tolerant flight control system.

Specifically, a parameter identification study was conducted on two research platforms to model the nominal dynamic behavior of the aircraft. On the WVU YF-22 platform, the dynamic behavior of the aircraft was modeled under both nominal and primary control surface failure conditions. In this study, several different parameter identification methods were utilized to arrive at optimized models for control law design and simulator development. To obtain the

nominal linear models, the Matlab ${ }^{\circledR}$ System Identification Toolbox was used to obtain baseline state space parameter estimates that were used as an initial point in an output-error optimization process. These optimized state space parameters were utilized for control law design. For the identification of the state space parameters under primary control surface failure conditions, the Fourier Transform Regression method was implemented. To identify the stability and control derivatives, the output-error optimization method was again implemented. With both aircraft 
platforms, the optimization procedure was enhanced using USAF DATCOM estimates. DATCOM was implemented by applying several assumptions based on aircraft geometry and flight Mach number to estimate some of the parameters for each platform. These estimates were used to provide bounds on individual parameters during stability and control derivative optimization to improve the results. Utilizing these different parameter identification methods together as well as implementing DATCOM to improve the optimization of the stability and control derivatives provided successful identification of parameters used for control law design and simulator development. 


\section{Literature Review}

The following sections review prior work relevant to the proposed research investigation on aircraft parameter identification for application within a fault-tolerant flight control system. Specifically, topics that are summarized include fault-tolerant flight control systems, aircraft parameter identification using DATCOM, parameter identification from flight data, and confidence interval analysis techniques. Each of these sections detail important studies utilizing techniques that were applied or adapted for the development of a detailed mathematical model of the WVU YF-22 and PACT aircraft.

\subsection{Fault-Tolerant Flight Control Systems}

Fault-tolerant flight control has applications in both manned and unmanned aircraft: it can be designed to assist a pilot in the occurrence of a structural, actuator, or sensor failure or the algorithms may be specifically crafted to apply to unmanned aircraft. Fault-tolerance may be defined as "a system's ability to maintain its functionality, even in the presence of faults", Additionally when applied to aircraft fault-tolerance may be stated as ${ }^{7}$ :

"The fail-safe design concept and techniques are used to ensure that, if any single element in a system or sub-system fails in any flight, such failure should not prevent the continuity of safe flight and landing, or significantly reduce the capabilities of the airplane. Thus, the application of the fail-safe design concept enables minimal occurrence and/or effects of failures, and provides protection against catastrophic failure conditions."

There are numerous methods that have been used in the development of fault-tolerant control laws for aircraft, and specifically within the fault detection and identification (FDI) research area. "Fault detection" is used to describe "the problem of making a binary decision either that something has gone wrong or that everything is fine" ${ }^{, 8}$. Methods such as the Integrated Multiple Model (IMM) Kalman Filter Approach, Artificial Intelligence techniques (i.e. neural networks and fuzzy logic), and adaptive control algorithms have all been utilized in some form to detect and accommodate for sensor and actuator failures on an aircraft. It may be noted that in order for these tools to be useful for fault detection that they should have the ability to detect the failure before the effects become severe or render the aircraft unrecoverable ${ }^{9}$. Difficulties in achieving this degree of reliability arise due to uncertainties in the system model and measurement noises 
or disturbances. Additionally, there is the possibility of false alarms or misdiagnosed fault detection that must be considered when designing such a flight control system ${ }^{9}$.

Methods for detecting and isolating a fault may be through hardware redundancy such as secondary actuator systems (although this is cost ineffective and leads to additional weight) or analytical redundancy, which requires a mathematical model of the aircraft. For analytical redundancy, quantitative models (state-space models) may be identified through parameter estimation techniques. For the quantitative model, a priori knowledge of the system is required, specifically under both nominal and fault conditions. With this approach, it should be known how the model parameters change for a given fault. A qualitative approach may be applied and used with artificial intelligence methods, where the model is used to predict the behavior of the aircraft in nominal conditions and under specific faults. The detection and isolation of the fault will then be established by comparing the predicted and actual behavior of the aircraft ${ }^{9}$.

The two basic approaches to fault-tolerant control are passive and active approaches. In the passive approach, robust control techniques may be applied to maintain a closed-loop response insensitive to certain failures. This technique is often restrictive as it depends on the robustness of the nominal closed-loop system and on the certain types of faults that it may account for. The active approach allows for the control system to be actively re-designed according to an estimation of the fault performed by the fault detection and isolation filter. This estimation may be handled by either an automatic on-line controller redesign, which has an on-line automatic controller that calculates new parameters upon the detection of a failure (adaptive control), or by selecting a new pre-computed control laws based on the failure that has been detected and its severity (hybrid control switching) ${ }^{9}$.

One study conducted through the NAWC-Aircraft Division Activity at Patuxent River, Maryland focused on a novel approach to sensor and actuator failure detection and identification and fault-tolerant control based on the Interacting Multiple Model (IMM) Kalman Filter approach. The IMM algorithm has similar structure to that of Multiple Model Adaptive Estimation (MMAE). In MMAE, the Kalman filter residuals are utilized to develop likelihood functions for different modes. These modes are then utilized as adaptive weighting factors to determine the mode probability ${ }^{10}$. With the IMM the initial conditions of each Kalman filter is mixed at each time step - essentially creating a dynamic interaction between each filter ${ }^{10,11}$. The 
robustness and simplicity of the IMM filtering structure has made it a desirable low-cost solution for real-time systems ${ }^{12}$.

In the study conducted at NAWC, only sensor and actuator failures were considered, as structural failures would require a different approach and was left for future consideration. The problem was formulated as seeking the optimal state estimate for a system whose dynamics may be represented by Equation $2.1^{13}$.

$$
\begin{aligned}
& x_{k+1}=A_{k}^{j} x_{k}+B_{k}^{j} u_{k}+w_{k}^{j} \\
& z_{k}=C_{k}^{j} x_{k}+D_{k}^{j} u_{k}^{j}+v_{k}^{j}
\end{aligned}
$$

With this formulation, there may be models $M^{j}$, where $M$ represents the model. The failures are then represented by a shift from one model to another, and the failures may be recovered by the system, such as a secondary sensor or unlocked actuator. The IMM filter is a technique that allows for rapid tracking of changes in a dynamic system, which makes it highly desirable for fault tolerant flight control law design for aircraft systems. With a state-space model representation of the aircraft parameters, the failure may be represented as a switch from a nominal model to a failure model. Since there will be several different types of possible failures considered for most systems, a separate model will be used as a representation of each failure type. Each state space model will be composed of a corresponding set of $A, B, C, D$, and covariance matrices. Specifically, an actuator failure corresponds to a change in the $B$ or $D$ and/or the covariance of the process noise $w$. With this IMM algorithm formulation, a priori knowledge is required about the UAV actuators and is used to develop a transition probability matrix. For this study, the actuator failure model implemented corresponded to a "floating" or zero-momentum failure where the surface snaps from the commanded position to a zeromomentum position and remains at this position. As an example, if a failure of the second actuator is to be modeled by a matrix $B_{a c t}$ with a null $2^{\text {nd }}$ column as represented by Equation $2.2^{13}$.

$$
B_{a c t}^{2 F}=\left[\begin{array}{ccccc}
b_{11} & 0 & b_{13} & \ldots & b_{1 m} \\
b_{21} & 0 & b_{23} & \ldots & b_{2 m} \\
\vdots & 0 & \vdots & \ldots & \vdots \\
b_{n 1} & 0 & b_{n 3} & \ldots & b_{n m}
\end{array}\right]
$$

This methodology was applied to a simulation study conducted for the Bell Helicopter Eagle-Eye tilt rotor UAV. The measured states considered for this study were the angular rates, Euler 
angles, ground speed components, altitude, inertial vertical velocity and acceleration, linear acceleration along the body axis, and true airspeed. For the actuator FDI, there were seven actuator inputs considered and derived on-line through mixing equations from the primary control inputs. These actuator inputs included the collective positions for the left and right rotors, the longitudinal cyclic positions for the left and right rotors, left and right flaperon deflections, and elevator deflection; therefore, there were seven actuator failure models utilized for this study. Each single failure was properly detected and identified, with the aircraft able to stabilize in all conditions. During simultaneous failure conditions (sensor and actuator failures), both failures were successfully detected and correctly identified, and the maneuver was successfully completed $^{13}$.

A case study conducted by the Control Group in the Department of Engineering at the University of Cambridge argued how Model Predictive Control (MPC) could be used to provide an effective fault-tolerant flight control system. Specifically, the researchers applied a MPCbased controller on a detailed nonlinear simulator model representative of the fatal crash of El Al Flight 1862, a Boeing 747-200F which lost two engines during takeoff from Schiphol Airport in Amsterdam ${ }^{14}$.

During the development and execution of this simulation study, some important basic assumptions were used. Statistics showed that in the period between 1990 and 1999, of the 421 fatal accidents involving large jet aircraft, not a single one was caused by loss of a pilot. From this statistic, the researchers assumed that the pilot would be available to fly the aircraft. The goal was then to develop a fault-tolerant flight control system that would be used with a pilot in the loop and have the controller maintain "pilot fly-ability". A second assumption was that there was a readily available fault detection and identification component available for use with the controller. Figure 1 outlines the basic fault-tolerant controller design used for this study ${ }^{14}$. 


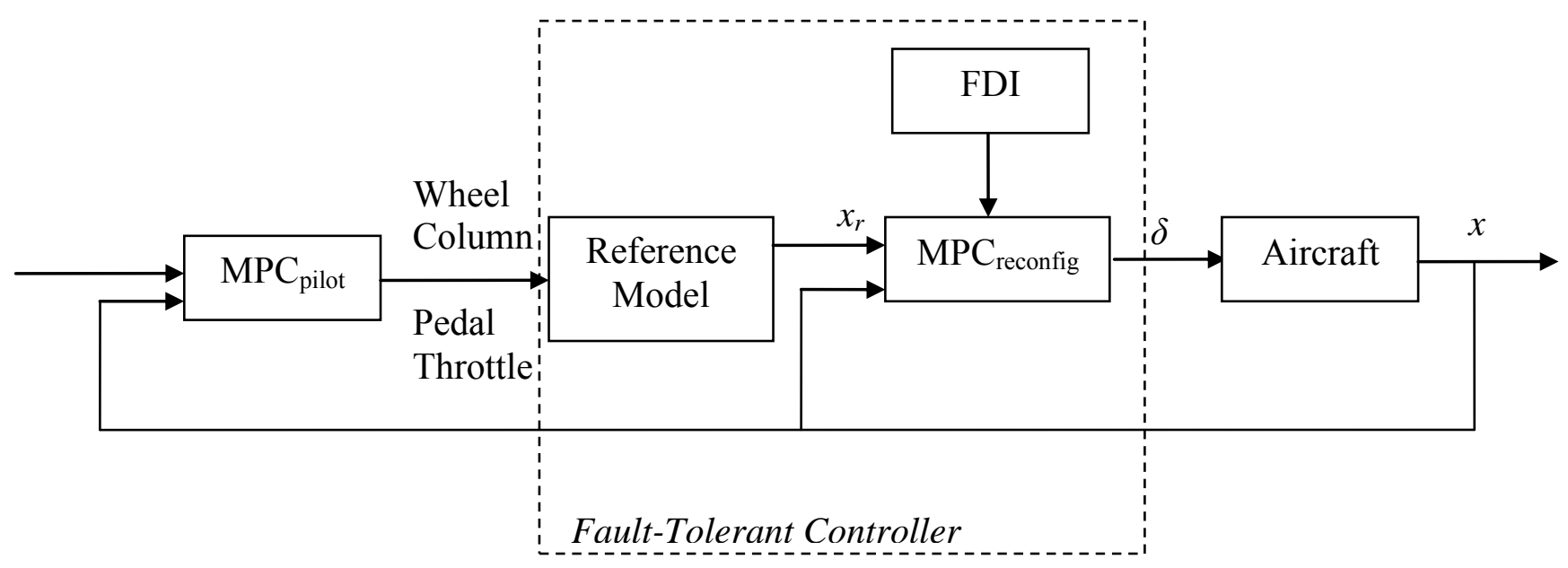

Figure 1: MPC Fault-Tolerant Controller [Reproduced from Reference 14]

The fault-tolerant controller consists of three components: the FDI, the reference model, and the

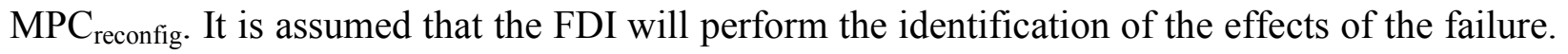
The reference model uses the pilot commands to generate a trajectory for the state vector of the aircraft, and the MPC controller which tracks the reference trajectory using the FDI output to update its internal model constraints. The MPC controller will select an input sequence that minimizes the error between the predicted future trajectory (determined by the reference model under assumption of constant pilot inputs) and the aircraft predicted trajectory (determined from FDI model). This form of model matching then was applied to the simulation of the incident of El Al Flight 1862. The $\mathrm{MPC}_{\text {pilot }}$ block is a representation of the human pilot for the simulation study $^{14}$.

El Al Flight 1862 took off from Schiphol Airport on October 4, 1992 and suffered a twoengine separation on its right wing. The pilots continued to control and fly the aircraft for 15 minutes following the failure before crashing into a building. This particular case study was considered due to the fact that the pilots were able to control this aircraft during this failure despite the large amount of damage to the aircraft. The right wing leading edge was seriously damaged, with the leading edge flaps partially removed. The right outboard aileron was unavailable for control, and six of the ten spoilers on the right wing were missing. Additionally, the effectiveness of the right inboard aileron was significantly reduced due to the air flow disturbance caused by the damaged leading edge and pylon loss ${ }^{14}$. 
American Airlines Flight 96 took off from Detroit Metropolitan Wayne County Airport on June 12, 1972 and suffered a cargo door failure due to an improper latching of door mechanism. A quick decompression of the cargo hold caused the cabin floor to partially collapse and jam some of the cables used to control the hydraulic actuators in the tail. The rudder jammed to its full right position and the tail-mounted \#2 engine of the McDonnell Douglas DC-10 shut down due to the severing of the control cables. Since the hydraulic lines were not severed, the pilots were able to utilize the ailerons, right elevator, and horizontal stabilizer ${ }^{15}$. The pilots were able to safely land the aircraft back in Detroit after correctly identifying the failures and utilizing the other available surfaces and engines to control the aircraft.

For the fault-tolerant MPC design, the inner loop shown in Figure 1 was designed to run at $10 \mathrm{~Hz}$, which is faster than the quickest mode on the Boeing 747. The $\mathrm{MPC}_{\text {pilot }}$ representing the pilot in the simulator was set to run at $1 \mathrm{~Hz}$. The inner loop was tuned to track the velocity, angle of attack, sideslip angle, and the angular orientation of the reference model. The control surfaces were constrained in magnitude and rate by physical limitations under the failure conditions. The simulations were conducted for both the nominal and failure nonlinear models using the same controller. The controller was designed to coordinate between the longitudinal and lateraldirectional dynamics due to the asymmetric nature of this failure. Although the simulation proved to be successful in that the aircraft was able to be brought to a safe landing on the runway, the authors do not make specific claims to have solved an entire class of fault-tolerant control law design issues. The MPC controller was tuned specifically for this failure, and was a very cumbersome process. The authors also maintain that since the tuning process was lengthy, it was not something that could be conducted quickly on-line. This specific failure allowed for sufficient time for tuning, but this application would be limited to less severe failures. The authors argue that they demonstrated the MPC as a plausible "implementation architecture"; however, unless a set of controller parameters are found to be generic for a larger array of fault conditions, than this will remain a restricted solution ${ }^{14}$.

A study at West Virginia University looked at the application of a restructurable flight control systems as a class of fault-tolerant control laws. The goal was to develop a fault-tolerant flight control system with application to unmanned aerial vehicles to perform sensor and actuator failure detection, identification, and accommodation (SFDIA/AFDIA). When observing actuator failures, locked actuators and damaged surfaces were considered. A coupling of the longitudinal 
and lateral-directional dynamics resulted from these actuator failures, and had to be considered when developing a model to use within the flight controller ${ }^{16}$.

For the restructurable flight control system, it was assumed that the failures would still leave the aircraft controllable, that is, the failures could be accommodated for using other available control surfaces or thrust vectoring. For restructurable control systems, the control laws may be reconfigured on-line, which will require increased computational power for the onboard computer. Figure 2 shows the basic block diagram of the fault-tolerant flight control system designed for this simulation study ${ }^{16}$.

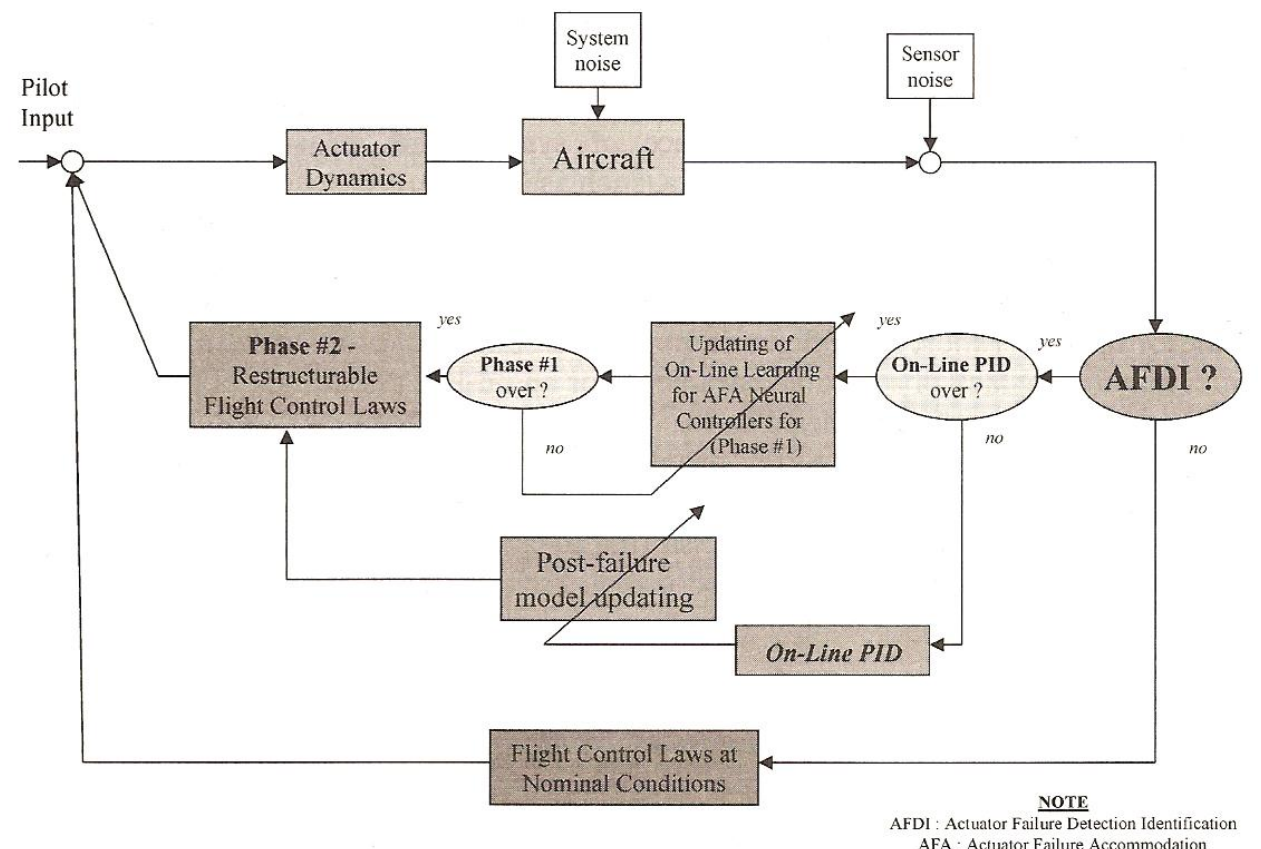

Figure 2: General Block Diagram for Fault-Tolerant Flight Control System [Reproduced from Reference 16]

For the development of these restucturable flight control laws, an on-line parameter estimation technique in the frequency domain was used to quickly identify and adapt the aircraft model as the actuator failures developed.

Another study conducted at West Virginia University aimed to develop a modeling and simulation environment for the NASA Intelligent Flight Control System F-15 aircraft. In this study, a simulation environment was developed to test flight control laws designed to handle control surface blockages and surface damage to the F-15 aircraft. An aircraft model with simulation capabilities for actuator failures was used with an optimal controller designed with both feedforward and feedback approaches ${ }^{6}$. Addtionally, this model had the capability for online parameter estimation using the Fourier Transform Regression method ${ }^{17}$. 
The flight control laws were designed using the Stochastic Optimal Feedforward and Feeback Technique (SOFFT) ${ }^{17,18}$, a set of control laws developed to achieve not only desirable handling qualities under nominal conditions but good post-failure flight conditions as well. For this study, two versions of the SOFFT controller were implemented: a decoupled version that consists of separate longitudinal and lateral-directional controllers and a version with a single controller to improve the aircraft handling at post-failure conditions. Within the SOFFT controller, the feedback and feedforward components were treated independently, with each designed to minimize a separate cost function. The feedforward control law was designed to have a "plant model" follow the dynamics of a "command model" by using a tracking technique. The feedforward calculated the control matrices by zeroing the derivative of the calculated error between the two models - a dynamic inversion approach - while The feeback part utilized an algorithm designed to minimize the integral of a quadratic cost function ${ }^{6}$.

Although the base goal of this study was to show that the modeling approach for failures on primary control surfaces could be conducted efficiently on-line and utilized within a faulttolerant flight control system, this study emphasized how vital it is for an accurate reference model to work in conjunction with a flight control system ${ }^{19,20,21,22}$. The simulation environment provided a tool to analyze the performance of the fault-tolerant flight control laws prior to being tested onboard the NASA F-15 manned aircraft.

The Self-Designing Controller (SDC) ${ }^{23,24,25,26}$ was a milestone in reconfigurable flight control where an on-line controller was utilized to avoid the necessity of having a priori assumptions regarding the nature of certain potential failures. The removal of the a priori control mode knowledge requirement reduced the development cost of the flight control platform. The SDC was designed under the goal of improving system performance under abrupt or gradual system changes. In 1996 this indirect-adaptive approach to reconfigurable flight control achieved "improved, appropriately decoupled responses during arbitrary effector or airframe impairment scenarios, and successful SDC flight tests culminating with a smooth landing of the VISTA/F-16 aircraft in cross-wind conditions with a (simulated) missing primary control surface (left horizontal tail) ${ }^{26}$. The SDC essentially utilized model-following receding-horizon optimal control paired with on-line parameter identification. The on-line parameter identification algorithm provided accurate estimates of time-varying parameters, under periods of both high and low excitation. Least-squares parameter identification was used with spatial and temporal 
constraints for the stability and control derivative estimation required to solve the optimal control problem $^{24}$. The SDC computed a time-varying model of the aircraft dynamics under the current conditions and with the on-line identification technique was able to track the parameters and be robust to these low-excitation conditions ${ }^{26}$. Figure 3 shows the general layout of the SDC.

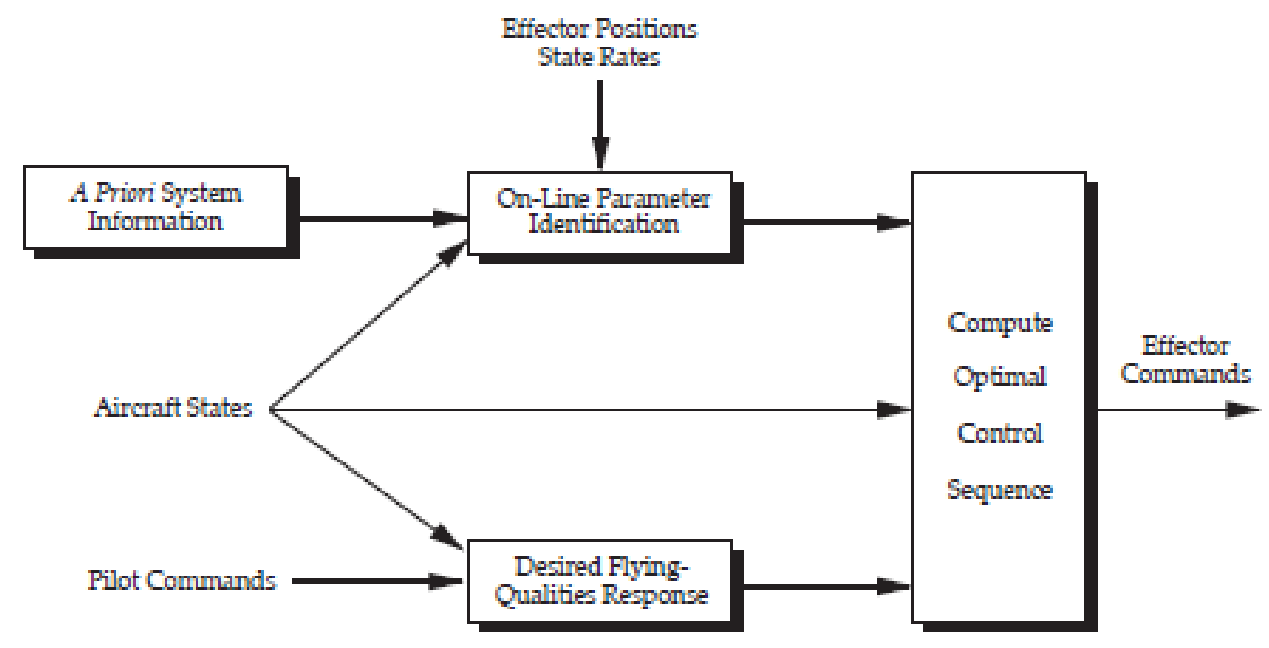

Figure 3: Self-Designing Controller Architecture [Reproduced from Reference 26]

Following the success of the SDC, another program focused on the development of the reconfigurable control systems enjoyed success in demonstrating adaptive capabilities.

The Reconfigurable Control for a Tailless Fighter Aircraft (RESTORE) $\operatorname{program}^{27,28,29,30,31,32,33}$ aimed to develop a reconfigurable/adaptive control law design to control an aircraft with little to no vertical tail effectors. Initial simulation studies utilized "algorithms for adaptive control, on-line real-time parameter estimation, and on-line constrained optimization for on-line control allocation, axis prioritization, and command limiting ${ }^{27, "}$. For the nonlinear simulation analysis, a reconfigurable control law based on "dynamic inversion in an explicit model following framework" utilized a neural network to adaptively control the error in the plant inversion ${ }^{27}$. The plant error was caused by failures, damage to the aircraft, and/or modeling uncertainties and the on-line control allocation was used to generate commands being sent to individual control surfaces to achieve the desired angular accelerations and optimize performance. The dynamic inversion was selected due to its common use for aircraft flight control design and for the ability to accommodate for nonlinear dynamics through gain 
scheduling and directly tune flying qualities based on the desired dynamics ${ }^{27}$. Through this simulation study, the reconfigurable flight control system yielded stable responses and strong command following performance following a rapid adaptation under critical failure and damage conditions $^{27}$.

The Air Force Research Lab (AFRL) was contracted by the Boeing Company to flight test reconfigurable/damage adaptive flight control laws on the X-36 Agility Research Test Aircraft $^{34,35}$. The RESTORE software was used to demonstrate adaptability of a neural network to compensate for control-surface actuator failures and/or damage without utilizing parameter identification. The removal of the parameter identification step eliminated the delay required during the learning process to produce new gains based on the newly-estimated model. Instead, stability margins were calculated in real time through the use of chirp signals sent to the actuators and utilizing the Fast Fourier Transform (FFT) on gyro telemetry data ${ }^{34,35}$.

Other studies in fault-tolerant flight control system development methods have been conducted by researchers. A team of researchers at the University of Missouri-Rolla have designed and implemented a reconfigurable flight control system on a 30\% scale model of a Cessna 150 and analyzed the aircraft performance ${ }^{36}$. At Georgia Institute of Technology, researchers have utilized several UAV platforms for a multitude of efforts, including the guidance, navigation, and control of rapid response unmanned rotorcraft envisioned for military and civilian reconnaissance missions ${ }^{37}$. Researchers at Massachusetts Institute of Technology have utilized UAV platforms for health management of a group of aircraft with application to persistent aircraft surveillance ${ }^{38}$. The Stanford University Dragonfly UAV Program has used two fixed-wing aircraft as a testbed for experimentally validating "hybrid control of single vehicles and real time danger zone computation and avoidance for two automated vehicles flying in parallel $^{\text {,39 }}$. NASA has utilized the AirSTAR UAV platform for research within the Integrated Resilient Aircraft Controls (IRAC) project, which is part of the NASA Aviation Safety Program. This extensive effort focuses on developing adaptive control algorithms for transport-sized aircraft experiencing adverse flight conditions ${ }^{40}$.

\subsection{Aircraft Parameter Identification through DATCOM Analysis}

A number of techniques within design approaches to fault-tolerant flight control systems rely on system and/or parameter identification. Wind tunnel and, in more recent years, CFD analysis have been recognized to provide the best estimates for all the aerodynamic coefficients. 
However, in addition to other drawbacks, both of them involve substantial costs and are not recommended earlier in the design stage. An appealing compromise between the very basic "empirical" approach and the accuracy of the comprehensive but expensive wind tunnel and CFD analysis is represented by the direct use of DATCOM or DATCOM-related methods. DATCOM $^{41}$, which abbreviates DATa COMpendium, is an extensive publication which correlates massive wind tunnel results mainly obtained by the US Air Force and NASA (previously called NACA) in analyzing virtually all possible aircraft configuration at subsonic, transonic, and supersonic speeds. DATCOM has represented an extremely valuable tool used in the early design phases of military and civilian aircraft with wind tunnel analysis (later followed by CFD analysis) for final validation and verification of the aerodynamic coefficients. DATCOM results have also been summarized in by Jan Roskam ${ }^{42}$. In more recent years some of the aerodynamic tools provided in DATCOM have been packaged within user-friendly codes which calculate the aircraft aerodynamic coefficients - and therefore the aerodynamic forces and moments - for a given specified aircraft geometry. The most relevant examples of these sophisticated but yet user-friendly computer codes are the "Advanced Aircraft Analysis" (AAA) - developed by DARCorporation ${ }^{43}$ - and "SimGen" - developed by Bihrle Applied Research ${ }^{44}$. Both codes provide essentially all the tools necessary for the complete aircraft design. In 1979, DATCOM was rewritten in FORTRAN IV computer language and appropriately named the USAF Stability and Control Digital DATCOM ${ }^{45}$.

DATCOM was originally developed with an application to manned aircraft, typically of a larger scale. The application of DATCOM to small unmanned aerial vehicles has recently become an interest among researchers who utilize these platforms for flight testing-related activities and flight simulations. Specifically, many of these researches must derive an accurate mathematical model of the UAV platforms for flight simulation and control law development ${ }^{46}$. Recently, DATCOM has been adapted into a digital version - appropriately named Digital DATCOM - where the user may input the aircraft geometric parameters, flight condition, and mass properties to obtain the aerodynamic coefficients.

Researchers at Georgia Institute of Technology ${ }^{47}$ utilized Digital DATCOM to input parameters for a $1 / 5$ scale Decathalon, shown in Figure 4. The research team implemented the geometric properties, general flight conditions, mass properties, and inertial properties obtained from a torsional pendulum setup into the program. 

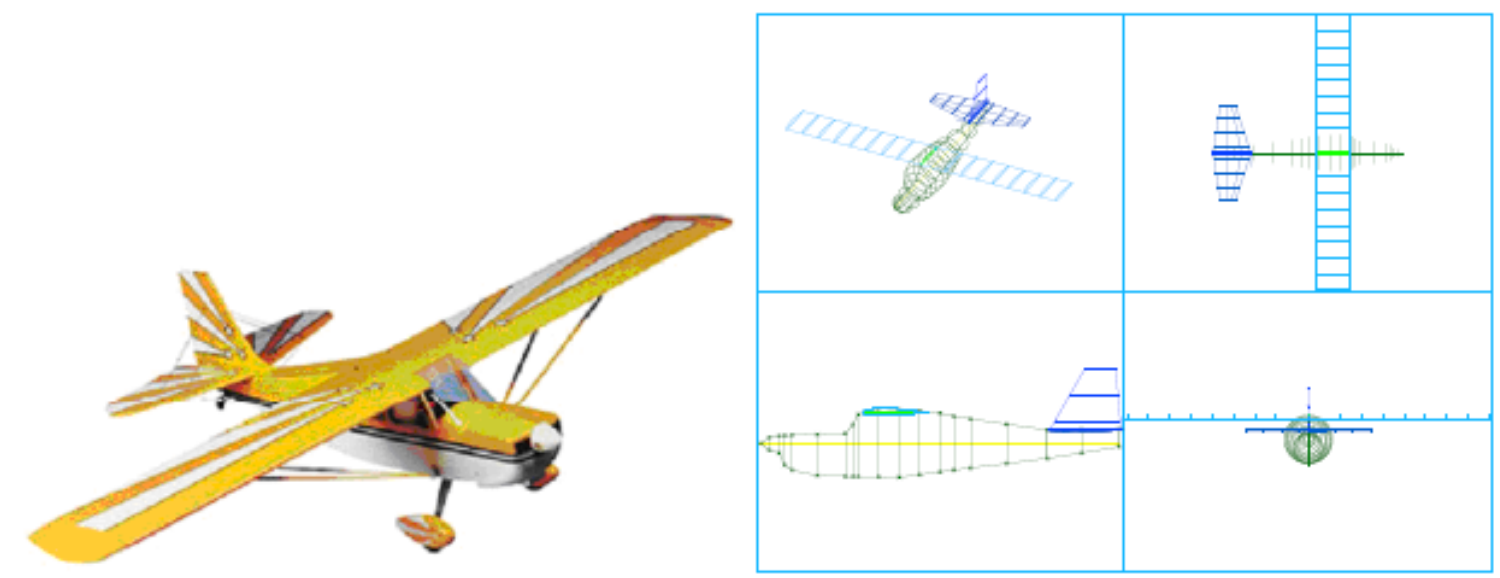

Figure 4: 1/5 Scale Decathlon R/C Aircraft and Digital DATCOM Representation [Reproduced from Reference 47]

Digital DATCOM was used to calculate the static and dynamic stability derivatives and the control derivatives for the aileron and elevators. The control derivatives for the rudders and the stability derivatives associated with the yawing moment were not calculated using DATCOM for this study. Flight tests with the Decathlon were used to collect data for parameter identification. During the flight tests, doublet maneuvers were injected by the $\mathrm{R} / \mathrm{C}$ pilot on the control surfaces to excite the aircraft dynamics with the assumption that the longitudinal and lateral-directional dynamics were uncoupled. A comparison between the stability and control derivatives obtained through PID and DATCOM was conducted with errors between the derivatives ranging from $4.5 \%$ for $c_{Y_{\beta}}$ to $16.8 \%$ for $c_{l_{\delta A}} 47$.

Orr et $\mathrm{al}^{48}$. sought to develop and evaluate control algorithms through simulation software for micro-aerial vehicles (MAV) in addition to small UAVs. An accurate model of the MAV had to be implemented into the simulation environment for control algorithm evaluation, so Missile DATCOM $^{49}$ was utilized to estimate the aerodynamic coefficients of the aircraft. Digital Missile DATCOM, which uses the component build-up method was originally developed for identifying the aerodynamic properties of tactical missiles, but its accuracy has been proven on several air and underwater vehicles ${ }^{48}$.

A generic MAV design was used for estimation via Missile DATCOM. The fuselage, wing, and tail effects were calculated independently and applied in conjunction with appropriate downwash effects to describe the MAV characteristics. The aircraft geometric and inertial properties as well as cruise velocity were implemented in this study. The average Reynolds 
number considered was 127,000 at sea level, and the MAV was given a maximum mass of 400 grams. These values were selected to relate the MAV to the small UAV regime for DATCOM calculations ${ }^{48}$. The generic model designed for use with DATCOM is shown in Figure 5.

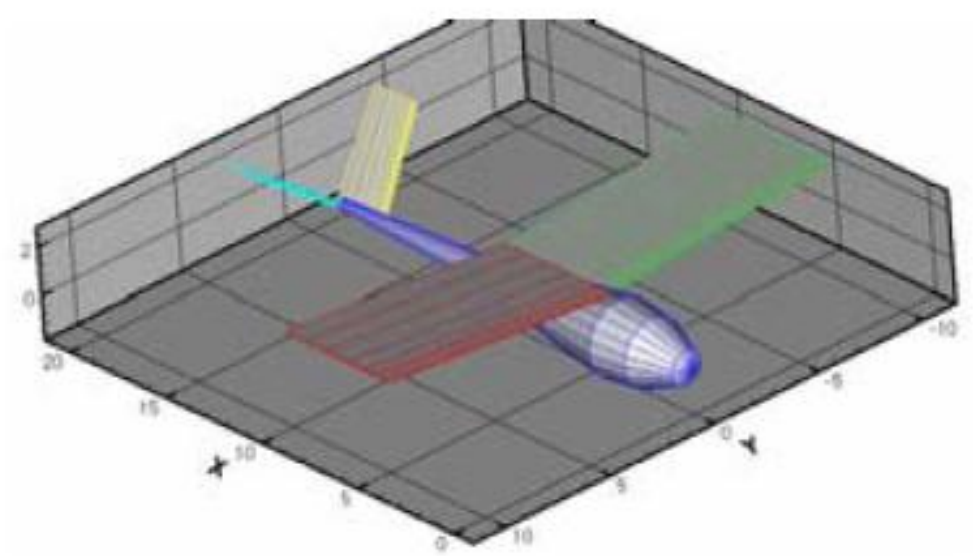

Figure 5: Generic MAV Model for use with Missile DATCOM [Reproduced from Reference 48]

This simplified model included straight, unswept planform wings with constant chord, no twist, and no control surfaces, and the fuselage is comprised of basic cylindrical sections. The V-tail includes two "ruddervators" - the sole control effectors of the aircraft. For the DATCOM estimation of the wings, a NACA-65A001 airfoil was used due to issues matching data with significantly thin, highly cambered airfoil sections. Additionally, Orr et al. wrote a Matlab ${ }^{\circledR}$ function to modify the DATCOM data sets to more accurately reproduce the observed behavior of the MAV model ${ }^{48}$.

The model obtained from Missile DATCOM was compared to results obtained from HASC flight data as well as wind tunnel data from flexible-winged aircraft model ${ }^{48}$, with $c_{l_{\beta}}$ showing the greatest discrepancy between the data sets at $50 \%$ and $83.3 \%$ error. For the final simulation model, Orr et al. relied solely upon the Missile DATCOM results, since the wind tunnel tests were not directly related to this aircraft configuration.

A study at Brigham Young University ${ }^{50}$ incorporated DATCOM to predict the natural frequencies, damping ratios, and time constants of the dynamic modes (excluding spiral mode) for three small UAVs - the Zagi 400, StablEyes, and the Procerus prototypes. Figure 6 shows the StablEyes prototype ${ }^{50}$. 


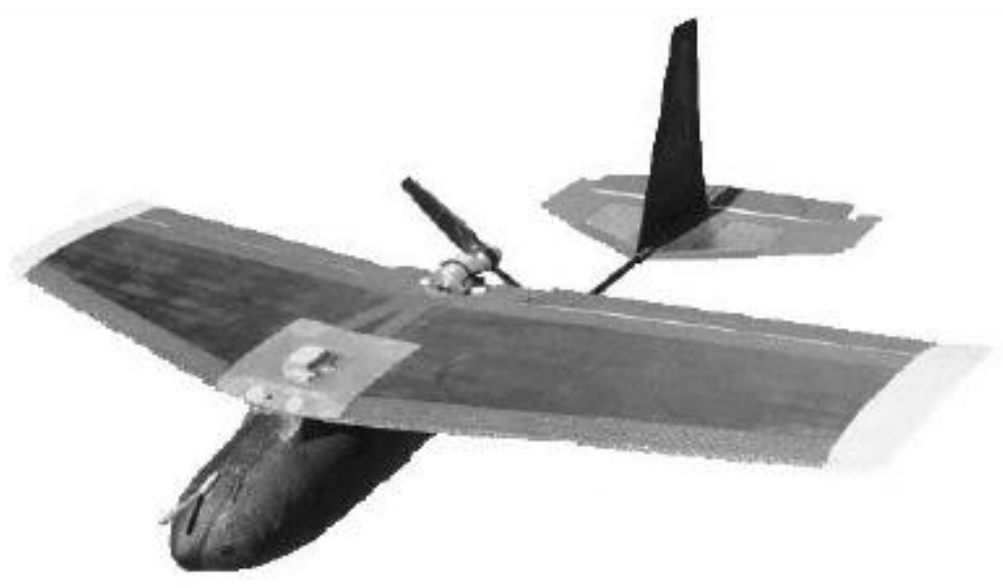

Figure 6: StablEyes Small UAV Prototype [Reproduced from Reference 50]

An Excel program - Dynamic Modes Predictor - was developed as an interface in the design process, to assist in applying the DATCOM methods to the small UAVs. Specifically, the geometric and inertial properties of the aircraft were implemented into the Dynamic Modes Predictor, which prompts the user to utilize a series of charts and graphs from Roskam I-VI ${ }^{42}$. The calculated values in the Dynamic Modes Predictor prompts the user to refer to specific charts listed in DATCOM. The spreadsheet provides the page and figure numbers of the charts and graphs to which the program is referring. After the values obtained from Roskam I-VI and DATCOM were implemented into the spreadsheet, the non-dimensional stability derivatives were generated. The mass, inertial, and velocity information implemented by the user was then used with the non-dimensional stability derivatives to determine their dimensional counterparts. The dynamic modes were predicted using the dimensional stability derivatives for the three UAV prototypes. To provide a measure of truth, the values for a full-scale Cessna 182 were implemented into the program and compared to the actual dynamic modes of the aircraft. Table 1 provides the results obtained by DAR Corporation using the Advanced Aircraft Analysis (AAA) Program $^{42}$ versus the results obtained from the Dynamic Modes Predictor ${ }^{50}$. 
Table 1: Comparison of Program Outputs for a Cessna 182 from Advanced Aircraft Analysis and the Dynamic Modes Predictor [Reproduced from Reference 50]

\begin{tabular}{|c|c|c|c|}
\hline Cessna 182 & AAA & $\begin{array}{c}\text { Dynamic Modes } \\
\text { Predictor }\end{array}$ & Percent Error \\
\hline$\omega_{\mathrm{n}, \mathrm{sp}}(\mathrm{rad} / \mathrm{s})$ & 5.27 & 6.39 & 21.3 \\
\hline$\zeta_{\mathrm{sp}}$ & 0.844 & 0.860 & 1.9 \\
\hline$\omega_{\mathrm{n}, \mathrm{ph}}(\mathrm{rad} / \mathrm{s})$ & 0.171 & 0.211 & 23.4 \\
\hline$\zeta_{\mathrm{ph}}$ & 0.129 & 0.075 & 41.9 \\
\hline$\omega_{\mathrm{n}, \mathrm{d}}(\mathrm{rad} / \mathrm{s})$ & 3.24 & 2.04 & 37.0 \\
\hline$\zeta_{\mathrm{d}}$ & 0.207 & 0.303 & 46.4 \\
\hline $\mathrm{T}_{\mathrm{s}}(\mathrm{s})$ & 55.9 & 1.83 & 96.7 \\
\hline $\mathrm{T}_{\mathrm{r}}(\mathrm{s})$ & 0.077 & 0.079 & 2.6 \\
\hline
\end{tabular}

Table1 highlights the percent error between the two programs. The short period damping and the rolling time constant were predicted accurately, with $1.9 \%$ and $2.6 \%$ error, respectively. However, the short period natural frequency and phugoid, Dutch roll, and spiral properties were not predicted with the same level of accuracy ${ }^{50}$.

In another study, Captain Nidel M. Jodeh of the USAF sought to develop a small UAV research platform, and one of the initial steps in the project was to derive an accurate model of the UAV for implementation in a simulation environment. The aircraft selected for this project was a radio controlled SIG Rascal 110, which was equipped with a Piccolo II Autopilot controller. Digital DATCOM was employed to conduct the necessary modeling for the aircraft for the simulation environment ${ }^{45,46}$.

For this application, a straight-tapered or non-straight-tapered wing in a mid-wing configuration was assumed. The inputs into Digital DATCOM included the airfoil, geometric, and inertial properties as well as the flight condition. Conservative assumptions were made in implementing the airfoil properties into Digital DATCOM because the Rascal 110 has an E193 airfoil and the program only accepts NACA airfoils. Additionally, the fixed landing gears were not directly accounted for through Digital DATCOM but incorporated into the fuselage ${ }^{45}$. Figures 7 and 8 show the Rascal 110 and its geometric representation as an input into Digital DATCOM. 

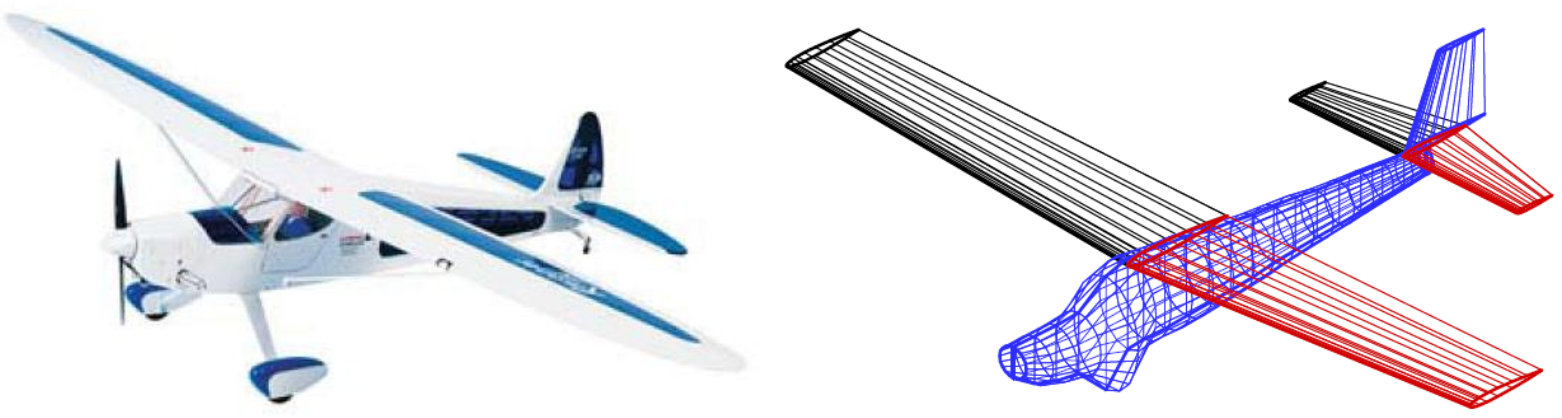

Figure 7: Rascal 110 Representation as Input to Digital DATCOM [Reproduced from Reference 45]
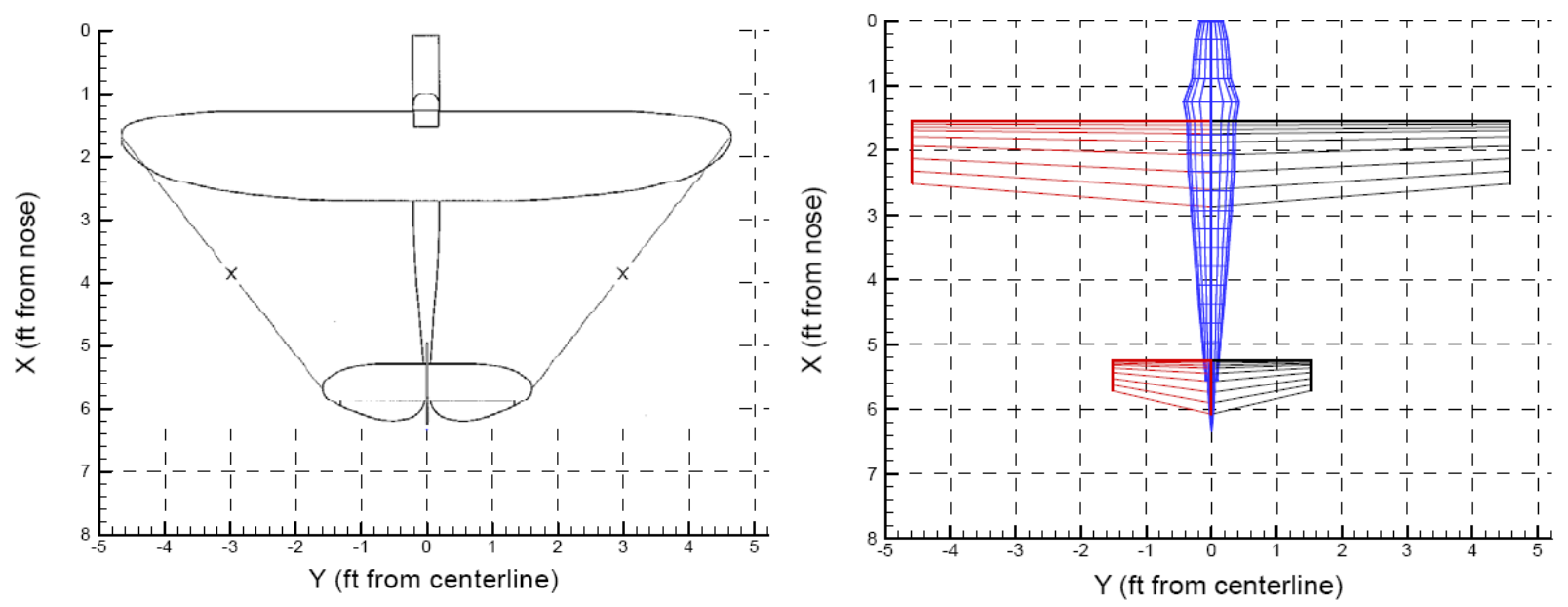

Figure 8: Rascal 110 Representation as Input to Digital DATCOM (Top View) [Reproduced from Reference 45]

The resulting stability and control derivatives from Digital DATCOM were used in the simulation environment for this study. Although several significant assumptions were made in implementing this aircraft into Digital DATCOM, the author believed them to be a good estimate of the aircraft properties. The author suggests performing flight tests in the future to validate the results obtained through the DATCOM modeling study.

A common issue with the efforts focusing on using DATCOM as a method for estimating the aerodynamic models of small UAVs is the inconsistency between the Reynolds numbers. The "conventional" subsonic aircraft methods employed in DATCOM do not directly translate to smaller-scale UAVs, posing the possibility of significant error. Additionally, many of the charts utilized in DATCOM for subsonic aircraft have data for Mach numbers above 0.2, a speed at which most of these UAVs are not reaching. 


\subsection{Aircraft Parameter Identification from Flight Data}

Although DATCOM and "Empirical" modeling techniques and wind tunnel tests are often used to identify the aerodynamic characteristics of aircraft, flight tests of the aircraft prototype are almost always carried out to verify these predictions through parameter identification. System identification is the determination of a dynamic system, often described in state space, given the inputs and the state outputs ${ }^{51}$. System identification is primarily focused on the determination of a mathematical model of the system, which is not unique. When referring to aircraft system identification, given the inputs and outputs, the aircraft dynamic system must be identified. Specifically, aircraft system identification is the modeling of the functional dependence of aerodynamic forces and moments on aircraft motion and control variables ${ }^{51}$. In many cases, the aircraft may be considered a rigid body so its motion can be modeled on the basis of Newtonian physics. With this assumption, system identification may be utilized to quantify the aerodynamic and thrust forces and moments acting on the aircraft ${ }^{51,52}$. When only estimating a state space representation or stability and control derivatives, this is often referred to as parameter identification.

Countless parameter identification ${ }^{53,54,55,56,57}$ studies from flight test data have been conducted on both manned and unmanned aircraft. The following paragraphs discuss some of these studies that utilized parameter identification techniques from flight data. More information describing aircraft parameter identification techniques can be found in Section 3 - Review of Parameter Identification.

In the mid 1960s, parameter identification in the time domain began being explored as an option for predicting and evaluating aircraft behavior at NASA Dryden Flight Research Center ${ }^{58}$. In the early 1960s there was a need to flight test the rocket-powered X-15 and the early liftingbody program to extract the aerodynamic stability and control derivatives from the measured flight data to expand their flight envelope. As the digital computer age began, major improvements were made to the parameter estimation process, specifically in the accuracy and efficiency of the curve-fitting of the time histories to predict the stability and control derivatives. Optimization techniques were developed using FORTRAN code that utilized the modified maximum-likelihood estimation (MMLE). As more and more flight-test programs were emerging in the late 1960s and early 1970s, this code was validated on a wide variety of aircraft in different flight regimes. Full parameter estimations were conducted at high angles of attack 
(above 40 degrees) using a 3/8 ${ }^{\text {th }}$ scale remotely-piloted F-15 model. The Gossamer Albatross, a human-powered vehicle, was analyzed in a flight regime with velocities around $15 \mathrm{~km}$ per hour, with the stability and control derivatives successfully identified. Parameter estimation analysis has been successfully conducted for vehicles flying in the transonic and supersonic regions, with speeds up to Mach 6. The F-8 Supercritical Wing program was used to determine lift and drag data using puss-over/pull-up maneuvers. Although Dryden briefly explored conducting PID in the frequency domain, the focus remained on time-domain maximum-likelihood efforts ${ }^{58}$.

Some major programs that have enlisted Dryden Flight Research Center to conduct parameter estimation to support flight testing include the X-29A, F-18 High Angle of Attack Research Vehicle (HARV) ${ }^{59}$, the SR- $71^{60}$, and the Space Shuttle Orbiter. The F-18 HARV was used for testing with the High Angle of Attack Technology Program (HATP), which began in the mid 1980s. The HARV program was divided into two phases: Phase I spanned over two years where high-AOA aerodynamics and handling behaviors were evaluated up to 55 degrees, and Phase II focused on hardware and software modifications to the HARV and spanned over 4 years. Within this Phase I, the stability and control derivatives were evaluated based on windtunnel data and preliminary flight tests by the US Navy. Initial PID analysis of the HARV revealed two primary issues in the analysis: the first was related to the aerodynamics due to unsteady separated and vortical flows when the aircraft performed over 20 degrees AOA, and the second issue was related to maneuvers being conducted with the control system engaged. With the first issue, the aircraft would exhibit uncommanded motions with varying frequency and amplitude. These motions were eventually accounted for by considering state noise in the modeling and assuming it for all PID maneuvers. The second issue made derivative extraction inaccurate due to the linearly dependent motions of the controls when flying above 25 degrees AOA. The feedback control system cause high correlations between the response variables and resulting control motions, which were complicated further because of their dependence on angle of $\operatorname{attack}^{58}$.

Within Phase II, a multi-axis thrust-vectoring control system (TVCS) and a specialized research flight control system (RFCS) were added to the HARV. The PID maneuvers (25 longitudinal and 26 lateral-directional) conducted during the test flights in Phase II were analyzed using the pEst program with state noise, which accounted for the uncommanded motions at high AOA. Maneuvers in Phase II were no conducted by a pilot as in Phase I, but by 
an onboard excitation system (OBES) while the aircraft was under the command of the RFCS. Specifically, the OBES contained preprogrammed maneuvers that were used for flutter envelope clearance, control power research, and aerodynamic and control law PID. The OBES would command individual control surface inputs through the RFCS, which eliminated control surface correlation issues. Additionally, the OBES allowed independent thrust-vectoring vane deflections (single-axis deflections), which used all vanes to excite responses in the pitch or yaw axis. The PID results for the longitudinal and lateral directional stability and control derivatives from Phase II were significantly improved from the Phase I derivatives. This was shown through tighter clustering of estimates and smaller uncertainty levels as established through Cramer-Rao bounds. The improvement in the estimates was attributed to the utilization of the OBES in producing repeatable maneuvers in the different flight regimes ${ }^{58}$. Figure 9 shows the F-18 HARV sideslip derivative as a function of AOA. The solid line represents the actual flight data, and the dashed line represents the prediction from PID.

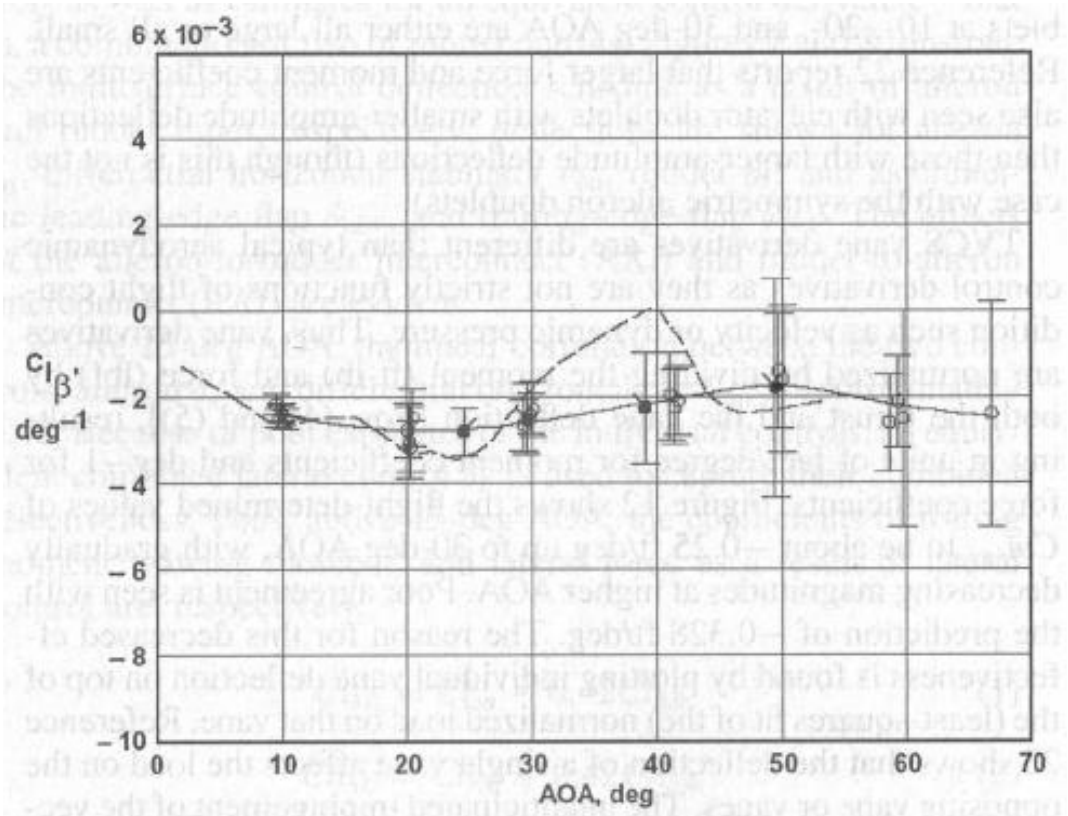

Figure 9: F-18 HARV Sideslip Derivative (Dihedral) as Function of Angle of Attack [Reproduced from Reference 58]

Although acceptable results were obtained for most of the stability and control derivatives for angles of attack less than 50 degrees, improvements in estimation could be made by collecting data at smaller AOA intervals (10 degree intervals were used in this study) because significant changes occur in aircraft behavior with 2 to 3 degree changes in AOA. Additionally, the input maneuvers could have been varied, i.e. doublet amplitude and frequency could have been 
adjusted in this study to observe the effects ${ }^{58}$. Overall, this project was a successful parameter identification study for an aircraft at high angles of attack utilizing the maximum-likelihood method, Cramer-Rao bounds, and an onboard excitation system.

Several other parameter identification studies ${ }^{61,62,63}$ have been conducted utilizing flight data collected from the HARV flight tests. In one study, researchers at WVU conducted a comparative study between wind tunnel estimates, the Maximum Likelihood estimation method, and results obtained using a frequency-based PID technique. Both longitudinal and lateraldirectional dynamics were analyzed for maneuvers performed at angles of attack of 20 degrees and 30 degrees. The Fourier Transform Regression technique was implemented using the Discrete Fourier Transform (DFT) and the Maximum Likelihood approach was coupled with the Newton-Raphson technique for its analysis. The frequency-based technique has potential for online applications due to its low processing requirements, so its results were compared to wind tunnel and Maximum Likelihood estimations for the stability and control derivatives. Many of the longitudinal and lateral-directional derivatives showed little error between the estimations, with the exception of $c_{z_{q}}$ which had a $327.6 \%$ standard error between the Maximum Likelihood and Fourier Transform Regression methods at an angle of attack of 20 degrees $^{61}$.

While there has been a lot of work with parameter identification and evaluating the accuracy of different methods, there has also been work on accounting for atmospheric turbulence and its effects on the accuracy of PID. A study at West Virginia University proposed a methodology for flight data reduction to account for non-homogenous atmospheric conditions ${ }^{64}$. The study was specifically applied to allow comparison between model-following adaptive control laws and implemented within a simulation environment and on flight data obtained by the WVU YF-22.

For this application an algorithm was developed for reduction of the flight data to account for varying atmospheric conditions. Essentially, the standard deviation of the measured angular rates during flight segments where there was no significant pilot input was represented as ${ }^{64}$ :

$$
\sigma\left(\hat{x}_{A(t)}\right)=\sqrt{\frac{\sum_{i}^{n}\left(\hat{x}_{A i}-\overline{\hat{x}}\right)^{2}}{(n-1)}}
$$


where $n$ indicates the number of samples. The subscript $A$ denotes that the controller was turned off and the nonlinear dynamic inversion control laws were activated. The same equation was repeated for a second flight test where the nonlinear dynamic inversion control laws augmented with neural networks was implemented with a subscript indication $B$. From these two estimations, the ratio of the standard deviations was given by:

$$
K_{t 1}=\frac{\sigma\left(\hat{x}_{B}(t)\right)}{\sigma\left(\hat{x}_{A}(t)\right)}
$$

It was shown that the following statement was true:

$$
\sigma\left(K_{t 1} \cdot \hat{x}_{A}(t)\right)=\sigma\left(\hat{x}_{B}(t)\right)
$$

The processed data was expressed as the following:

$$
K_{t 1} \cdot \hat{x}_{A}(t)=\hat{x}_{A}(t)+\Delta \hat{x}_{t}(t)
$$

where:

$$
\Delta \hat{x}_{t}(t)=\left(K_{t 1}-1\right) \hat{x}_{A}(t)
$$

Through the addition problem expressed in Equation 2.6, a similarity conversion is achieved relating the atmospheric turbulence between tests $A$ and $B$. Corrections were also applied for the measurements of $x_{A}(t)$ which accounted for the differences in atmospheric turbulence conditions:

$$
x_{A}^{(T C)}(s)=T F_{H Q}(s) \frac{T F_{A S}(s)}{1+T F_{A S}(s)} \delta_{s t A}(s)+\frac{T F_{\text {Sens }}(s)}{1+T F_{A S}(s)} \Delta x_{t A}^{(T C)}(s)
$$

where the following must be true:

$$
\sigma\left(\Delta x_{t A}^{(T C)}(t)\right)=\sigma\left(\Delta x_{t B}(t)\right)
$$

$\Delta x_{t A}^{(T C)}(t)$ may be evaluated using:

$$
\Delta x_{t A}^{(T C)}(t)=\Delta x_{t A}(t)+K_{t 2} \cdot \Delta \hat{x}_{t}(t)
$$

Equation 2.10 introduces a correction factor $K_{t 2}$ that must be determined so that the following is true:

$$
\sigma\left(K_{t 2} \Delta \hat{x}_{t}+\Delta x_{t A}\right)=\sigma\left(\hat{x}_{B}\right)
$$

where $\Delta x_{t A}(t)$ and $\Delta \hat{x}_{t}(t)$ are uncorrelated so: 


$$
K_{t 2}^{2} \cdot \sigma^{2}\left(\Delta \hat{x}_{t}\right)+\sigma^{2}\left(\Delta x_{t A}\right)=\sigma^{2}\left(\hat{x}_{B}\right)
$$

Assuming $\sigma\left(\Delta x_{t A}\right)=\sigma\left(\hat{x}_{A}\right)$ and if $\sigma\left(\hat{x}_{B}\right)>\sigma\left(\hat{x}_{A}\right)$, then the following expression for the correction factor may be used:

$$
K_{t 2}=\frac{\sigma\left(\hat{x}_{B}\right)+\sigma\left(\hat{x}_{A}\right)}{\sigma\left(\hat{x}_{B}\right)-\sigma\left(\hat{x}_{A}\right)}
$$

Using Equation 2.10, Equation 2.8 can be rewritten as:

$$
x_{A}^{(T C)}(s)=T F_{H Q}(s) \frac{T F_{A S}(s)}{1+T F_{A S}(s)} \delta_{s t A}(s)+\frac{T F_{\text {Sens }}(s)}{1+T F_{A S}(s)} \Delta x_{t A}(s)+K_{t 2} \frac{T F_{\text {Sens }}(s)}{1+T F_{A S}(s)} \Delta \hat{x}_{t}(s)
$$

It follows that the corrected measurement to account for differences in the atmospheric turbulence was expressed as:

$$
x_{A}^{(T C)}(t)=x_{A}(t)+K_{t 2} \cdot \Delta \hat{x}_{t}(t)
$$

This correction algorithm was implemented in simulator tests for the WVU YF-22 as well as on segments of flight data under the two different controller conditions. This data reduction algorithm addressed the issues with inconsistent flight test conditions due to differences in atmospheric turbulence and provided a performance comparison of the two different flight control laws. The corrections were implemented and were shown to provide identical standard deviations of the perturbations attributed to the atmospheric turbulence. This application was successfully integrated with data collected on the WVU YF-22 for the assessment of the augmented neural network effects on the nonlinear dynamic inversion-based flight control laws ${ }^{64}$. 


\section{Review of Parameter Identification}

The following subsections of Chapter 3 review the basics of aircraft parameter identification, including the definition of parameter identification and how it applies to aircraft. Within these sections, general experiment design with the goal of aircraft parameter identification is discussed, specifically indentifying typical maneuvers used for this process. Parameter identification methodologies in both the time and frequency domains are also reviewed.

\subsection{Aircraft Parameter Identification}

When developing a mathematical model of a system, the primary process being undertaken is called "parameter estimation," which refers to quantifying the model by applying a numerical, usually statistical, procedure ${ }^{65}$. Figure 10 shows a general dynamic system overview in block diagram format.

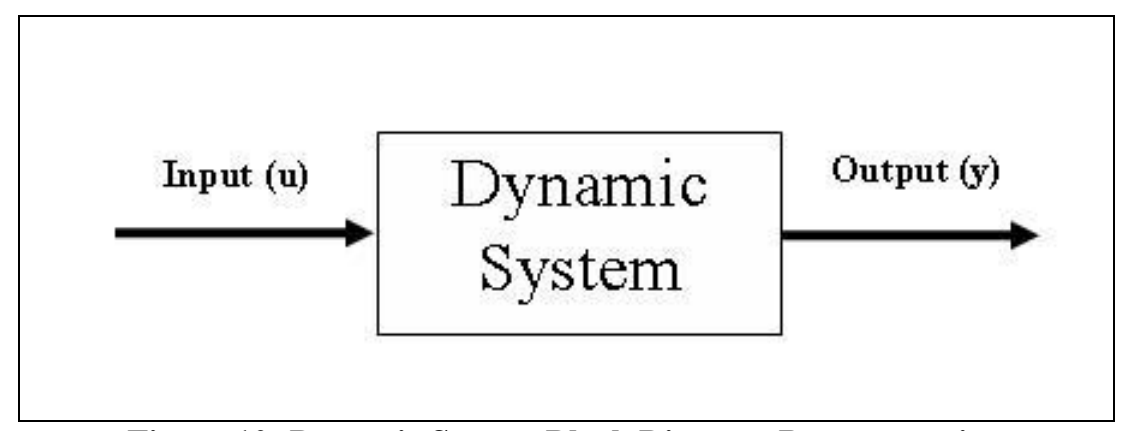

Figure 10: Dynamic System Block Diagram Representation

Three general problems arise in dynamic system theory ${ }^{51,65}$ :

1. The simulation problem: Given the inputs and the system, find the outputs.

2. The control problem: Given the system and the outputs, find the inputs.

3. The identification problem: Given the inputs and the outputs, find the system.

In this research project, the identification problem is addressed: inputs were provided to excite the aircraft dynamics, and the outputs representing the various states were recorded. These inputs and outputs were used to identify a model representing the system behavior. Although this model is not the "true" parameter model, it provides an accurate mathematical representation of the aircraft dynamic behavior.

Aircraft parameter identification may be conducted off-line or used in real-time applications. For off-line applications, a set of flight data is used to estimate the unknown parameters. In real- 
time applications, as the aircraft is flying, an on-line process is estimating the aircraft mathematical model based on its flight behavior. Off-line parameter identification may be accomplished using either time or frequency domain methods; however, in most real-time applications frequency domain methods are most often employed due to their lower processing power requirements ${ }^{51}$.

When designing a flight test experiment for parameter identification, it is important that the choice of the input signals is such that the data will be maximally informative. Additionally, once data has been collected and used for model identification, it is essential that the researcher is able to review the sets of derived models and select a "suitable one". This often requires $a$ priori knowledge of the system or engineering insight guided by experience. Determining the "best" model is driven by the quality of its performance when attempting to reproduce the measured data. Finally, the model must be validated through a series of procedures to assess how the model performs when compared to actual recorded data and how confident one can be in the results $^{66}$. Figure 11 shows a basic overview of the parameter identification process ${ }^{66}$. 


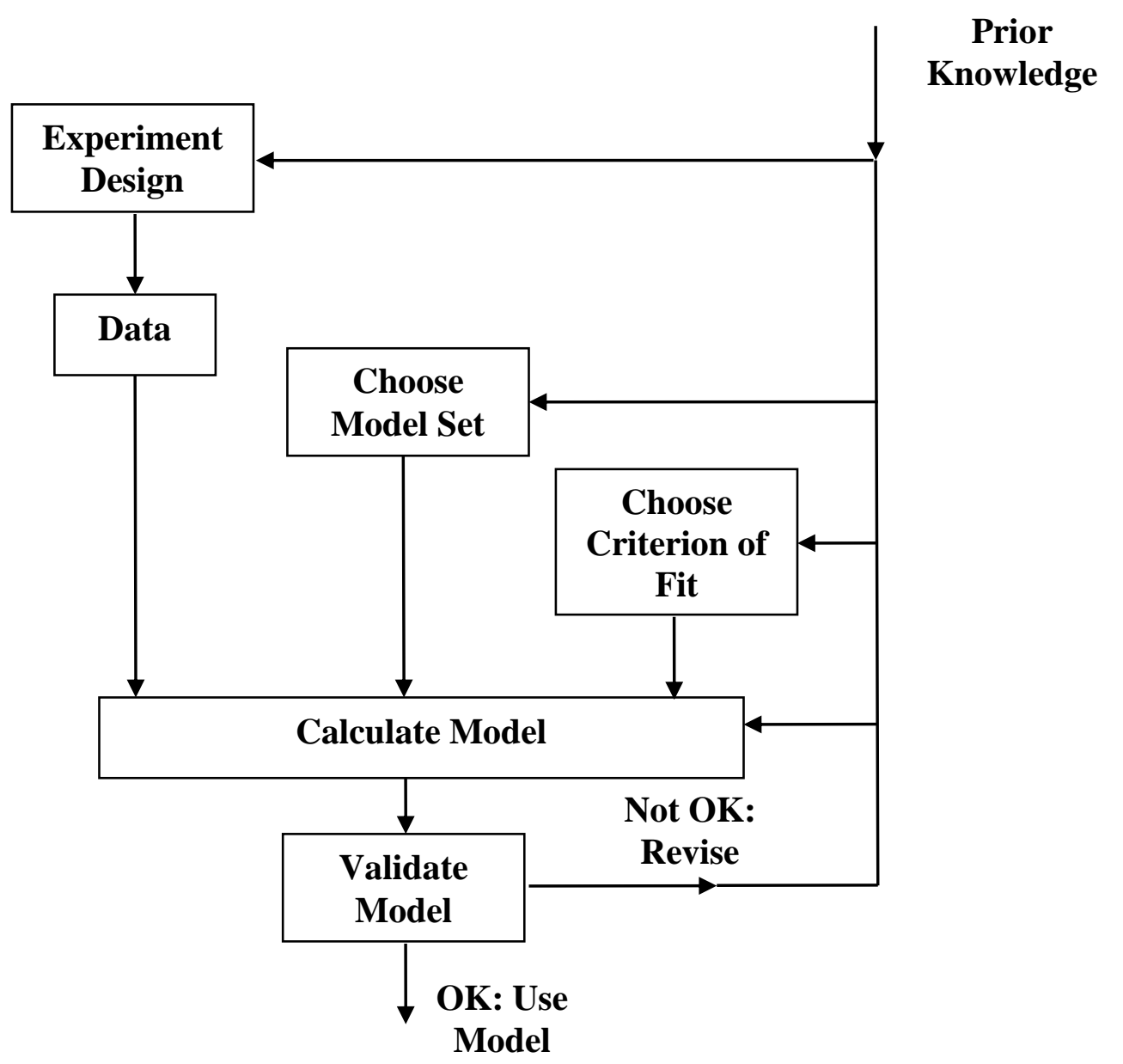

Figure 11: Parameter Identification Loop [Reproduced from Reference 66]

\subsubsection{Experiment Design}

Proper experiment design is a key element of any parameter estimation study because if sufficient and accurate data is not collected the system cannot be modeled. The quality of the data is the limiting factor in regards to both scope and accuracy for model development from PID. When applied to aircraft parameter identification, it is essential that the instrumentation and data acquisition system are appropriately selected, and the necessary flight conditions and maneuvers are implemented ${ }^{51}$. While attempting to maximize the amount of information in the collected data, there are several constraints that must be considered ${ }^{51}$ :

1. Limits on input/output amplitudes to ensure estimation can be conducted under linear conditions;

2. Limited resolution or range of sensors or data acquisition system; 
3. Consistent acquisition rate among all sensors;

4. Time constraints for each maneuver and entire flight test;

5. Limitations on control surface deflection rates and positions.

The quality of the measured flight data has a direct bearing on the accuracy of the estimated parameters. The following measured data channels are commonly used for complete aerodynamic model identification: control surface deflections, angular rates, linear accelerations, attitude angles, air data, and engine parameters. It is essential that the control surface deflections are measured accurately since they are the inputs to the aircraft model being identified. This data is typically collected through the use of potentiometers mounted directly to the control surface hinges ${ }^{65}$ or through measuring pilot input. Inertial measurement units (IMUs) usually provide angular rates as well as linear acceleration information for the aircraft and special attention must be paid to ensure that it is aligned with the aircraft body axes. If the IMU must be aligned off of the body axes, a correction factor must account for this discrepancy when handling the data. Standard air data probes often include frictionless veins to measure the aircraft angle of attack and sideslip angle in addition to a static pressure measurement. The attitude angles are not considered to be vital components when conducting a parameter identification study since the aerodynamic forces and moments are not dependent upon these angles and they are not utilized in least square algorithms ${ }^{65}$. If their measurement is desired for other research purposes, magnetometers and mechanical gyroscopes are two viable sensors to measure these states.

\subsubsection{Selecting Parameter Identification Maneuvers}

When designing an experiment for parameter identification (PID), the input maneuvers should be selected as to excite the appropriate states to be measured. Specifically, PID maneuvers for aircraft are designed to excite the longitudinal and lateral-directional dynamic responses through specially designed surface movements. These inputs determine the operating point of the system and which modes will be excited during the flight ${ }^{66}$. These maneuvers can vary depending on the needs of the researcher and the aircraft system, though some of the most common maneuvers include: doublets, multisteps, multiple-input, and frequency sweep maneuvers ${ }^{51,65}$. The general objective in exciting the aircraft modes properly is to specify the maneuver time length, the control surface to be moved, and the form of the input. There are typically two approaches that may be taken when selecting the type of maneuver to use to excite the aircraft dynamics. The first assumes no a priori knowledge of the system, so the input is 
designed to excite the system over a broad frequency range, i.e. using frequency sweeps. The second approach assumes $a$ priori knowledge of the dynamic behavior of the system so the input is designed accordingly. Typical inputs for this method include doublets at or near the natural frequency of the dynamic modes of the aircraft ${ }^{51}$.

As listed previously, there are limitations on the maneuvers performed for PID. Specifically, there are limits on maneuver amplitude and frequency that must be taken into consideration when designing an experiment, and specific aircraft response variables, such as angle of attack and angular rates, may need to remain within certain bounds. A serious limitation factor is also the amount of time available for an experiment, including the available window for performing a specified maneuver. For example, it is often difficult to perform maneuvers at a sustained high angle of attack, so the amount of time available to perform the maneuver and collect data is limited. Additionally, large output amplitudes over a longer duration would provide the most accurate parameter estimates; however, the amount of time necessary to perform that maneuver may not be available to the researcher. Inputs with a low-frequency could cause the aircraft to exit the flight condition desired for the maneuver, so this is another constraint that must be taken into careful consideration in the experiment design. Consideration must also be taken into the control surface actuator rate limits, which may interfere with the design of square-wave inputs if a sharp-edged maneuver is desired ${ }^{51}$.

The following paragraphs summarize selected PID maneuvers and the typical goals for their implementation, reviewing some of the issues and benefits associated with each maneuver. The maneuver types discussed are of both single and double input designs and include: impulse, doublet (single and multi-input), multistep, and frequency sweep.

Impulse maneuvers are considered the simplest inputs used for PID. The impulse consists of a spike, also known as a stick rap, used to excite the aircraft dynamics. The impulse may be a double-sided input to help return the aircraft to its initial flight condition. Since an impulse typically has low input energy (low amplitude) when observing its power spectrum, it is often only used for prediction cases where there is no a priori information on the aircraft ${ }^{51}$.

Doublet inputs are two-sided pulses, often with each pulse being symmetric in duration and amplitude as shown in Figure 12. When selecting the duration of the pulses the dominant frequency of the input should be at or close to the natural frequency of the system. The amplitude of the doublet should be selected so that the amplitude of the aircraft dynamic response is large 
enough to provide a good signal-to-noise ratio. The amplitude should not be too large to exceed a limit where the model parameters can no longer be considered constant, i.e. not to exit a given flight condition during the maneuver ${ }^{51}$.

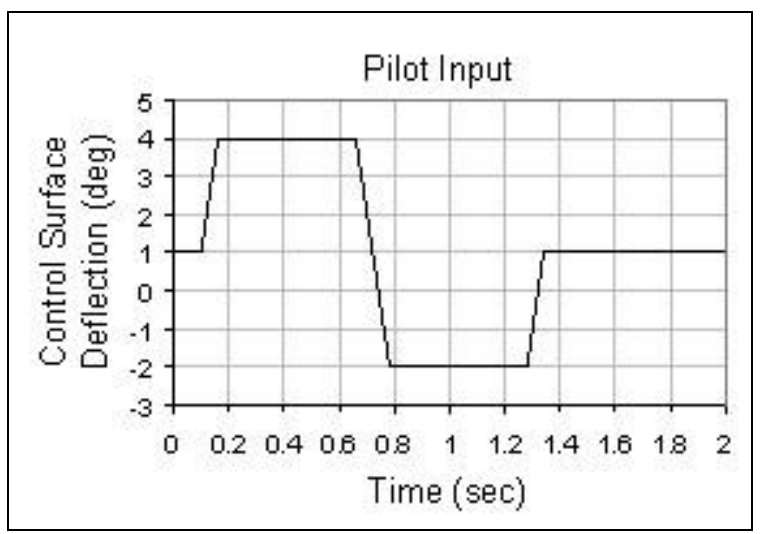

Figure 12: Typical Symmetric Doublet Maneuver [Reproduced from Reference 67]

The square-wave form provides an advantage over a single-frequency sinusoid input for parameter identification purposes in that the input spectrum is broadened. This is helpful in the event that a poor a priori estimate was established for the frequency of the dynamic mode being excited. The primary downfall of the doublet maneuver when used for parameter identification is that it excites a small frequency band, so when possible it is desirable to use a multistep or frequency sweep maneuver for more comprehensive coverage of the frequency spectrum ${ }^{51}$.

When modeling the lateral-directional dynamics, it is often necessary to use a multiple-input design. This typically involves rudder and aileron inputs to excite the yawing and rolling dynamics of the aircraft. When addressing multiple input designs, three additional aspects must be considered: relative effectiveness, coordination, and correlation. With relative effectiveness, the size of the control surface to be moved is taken into consideration. A larger control surface may require smaller movements to adequately excite the dynamics when compared to smaller control surfaces on the aircraft. This consideration is often addressed using a priori information from previous flight test experience with similar aircraft or wind-tunnel analysis. With coordination, the multiple inputs must be coordinated to maximize the data information collected, and to improve the maneuver through advantages of dynamic coupling in the aircraft motion. The most prevalent example of this type of maneuver is when a rudder/aileron combination doublet is performed to excite the Dutch roll and roll responses of the aircraft. Applying the rudder first is essential in this maneuver to collect sufficient data, as the Dutch roll is a slower mode than the roll. When applying the aileron following the rudder doublet, the roll 
mode is excited and additional excitation of the Dutch roll is performed. It is equally as important to manage the timing between the rudder and aileron doublets to collect useful data: the spacing between the doublets should be near the period of the Dutch roll. Finally, correlation refers to the similarity of the waveforms for multiple inputs. It is desirable to use input waveforms that are completely decorrelated to obtain accurate estimates of control effectiveness $^{51}$.

A multistep input is often a sequence of doublet maneuvers on a control surface with varying durations (widths) so to excite a larger frequency range. Common multistep maneuvers are the 32-1-1 and, conversely, the 1-1-2-3 maneuver. These types of inputs consist of alternating pulses with widths in the ratio of 3-2-1-1 or 1-1-2-3, respectively. It is desired that the width of the "2" pulse corresponds to half of the period of the natural frequency of the excited dynamic mode. With this design, the " 3 " and "1" pulses on either side of the "2" pulse "bracket" that frequency, creating a wideband input. It may often be desirable to use the 1-1-2-3 in practice because implementing the 3-2-1-1 can be difficult with the longest pulse at the start of the maneuver. This may cause the aircraft to deviate from the preferred flight condition. Another option is to perform a 2-1-1 input, which utilizes a slightly shorter multistep maneuver. In this case, the pulse widths are selected to "bracket" the expected natural frequency of the excited dynamic mode. Equation 3.1 has been shown to work well when determining the width of a pulse for the 2-1-1 maneuver ${ }^{51}$.

$$
1 \text { pulse width }=\frac{0.7}{2 f_{n}}
$$

A frequency sweep is a common maneuver utilized for PID when there is little a priori knowledge about the dynamic system. The goal of this type of input is to apply a continuous sinusoid input with a frequency increasing over time to cover a broad frequency band of interest. Since it is necessary for the frequency sweep to contain several complete cycles of a large number of frequencies across a desired band, a longer duration may be required to perform this maneuver. For example, to excite a band between 0.1 and $10 \mathrm{rad} / \mathrm{sec}$, each frequency sweep could take between 60 - 90 seconds. It is not essential for the frequency sweep maneuver to have constant amplitude, to have an exact frequency progression, or to be exactly repeatable. There are several issues involved with implementing the frequency sweep. These include the length of time required for successful, complete implementation and the fact that implementing low 
frequency content first may cause the aircraft to diverge from the desired flight condition. Additionally, a frequency sweep should only be implemented on one surface pair at a time, so multiple input maneuvers are not useful for modeling multiple input dynamics. Despite these downfalls, if there is sufficient time to implement a frequency sweep in a flight test, there will be comprehensive coverage of the desired frequency band and the accuracy of the identified model improved $^{51}$.

\subsubsection{Parameter Identification in the Time Domain - Output Error Method}

A common practice in PID is to determine model parameter estimates by maximizing a likelihood function (minimizing the weighted least-squares difference between measured and modeled outputs). To practically apply the maximum likelihood method, an assumption of no process noise is often made so that the states may be deterministically computed by direct numerical integration. This is the basis for the output-error method, which allows for parameter estimation of a deterministic linear dynamic system described by Equations $3.2-3.5^{51,65}$.

$$
\begin{aligned}
& \dot{x}(t)=A x(t)+B u(t) \quad x(0)=x_{0} \\
& y(t)=C x(t)+D u(t) \\
& z(i)=y(i)+v(i) \quad i=1,2, \ldots, N \\
& \operatorname{Cov}[v(i)]=E\left[v(i) v^{T}(j)\right]=R \delta_{i j}
\end{aligned}
$$

where $v$ has a normal distribution, $N(O, R)$. For this linear dynamic system, the unknown parameters are elements of the $A, B, C, D$, and $R$ matrices. Equation 3.6 shows the residuals or output errors, and Equation 3.7 shows the representation of the negative log-likelihood function ${ }^{51}$.

$$
\begin{gathered}
v(i)=z(i)-y(i)=z(i)-C x(i)-D u(i) \quad i=1,2, \ldots, N \\
-\ln L\left(Z_{N} \theta\right)=\frac{1}{2} \sum_{i=1}^{N} v^{T}(i) R^{-1} v(i)+\frac{N}{2} \ln |R|+\frac{N n_{0}}{2} \ln (2 \pi)
\end{gathered}
$$

where $Z_{N}$ is the likelihood function for a sequence of measurements $[z(1), z(2), \ldots, z(\mathrm{~N})]$. If the right-hand side of Equation 3.7 is differentiated with respect to $R$ and the result is set equal to zero, the result is the form shown in Equation 3.8. Typically only the diagonal elements of $R$ are estimated from this equation. 


$$
\hat{R}=\frac{1}{N} \sum_{i=1}^{N} v(i) v^{T}(i)
$$

For a given $\hat{R}$ the negative log-likelihood cost function becomes the form as shown in Equation 3.9. The negative log-likelihood cost function is minimized by calculating $\hat{R}$ from Equation 3.8 for a fixed $\theta$. The value for $R$ is then fixed to the value computed for $\hat{R}$, and the cost function in Equation 3.9 in minimized with respect to $\theta$.

$$
J(\theta)=\frac{1}{2} \sum_{i=1}^{N} v(i) \hat{R}^{-1} v^{T}(i)=\frac{1}{2} \sum_{i=1}^{N}[z(i)-y(i)] \hat{R}^{-1}[z(i)-y(i)]^{T}
$$

The last two terms in Equation 3.7 were dropped since they do not depend on the unknown model parameters. The goal of the output-error method is to minimize the negative loglikelihood cost function after solving for $\hat{R}$ using Equation 3.8. The optimization is better conditioned when it is completed with $\theta$ and $R$ adjusted alternately, and the steps are repeated until the convergence criteria are satisfied. The Newton-Raphson method may be used for the optimization of the cost function and the gradient of this function is obtained via Equation $3.10^{51}$.

$$
\frac{\partial J(\theta)}{\partial \theta_{j}}=\sum_{i=1}^{N} \frac{\partial v^{T}(i)}{\partial \theta_{j}} \hat{R}^{-1} v(i)=-\sum_{i=1}^{N} \frac{\partial y^{T}(i)}{\partial \theta_{j}} \hat{R}^{-1} v(i)
$$

Equation 3.11 represents the elements of the second-order gradient matrix. The second order partial derivative term is neglected, which results in the Gauss-Newton optimization algorithm. The second order gradient matrix is used to estimate the parameter vector change, as represented in Equation 3.12.

$$
\begin{aligned}
\frac{\partial^{2} J(\theta)}{\partial \theta_{j} \partial \theta_{k}} & =\sum_{i=1}^{N} \frac{\partial y^{T}(i)}{\partial \theta_{j}} \hat{R}^{-1} \frac{\partial y(i)}{\partial \theta_{k}}-\sum_{i=1}^{N} \frac{\partial^{2} y(i)}{\partial \theta_{j} \partial \theta_{k}} \hat{R}^{-1} v(i) \quad j, k=1,2, \ldots, n_{p} \\
\Delta \hat{\theta} & =\left[\sum_{i=1}^{N} \frac{\partial y^{T}(i)}{\partial \theta} \hat{R}^{-1} \frac{\partial y(i)}{\partial \theta}\right]_{\theta=\theta_{0}}^{-1}\left[\sum_{i=1}^{N} \frac{\partial y^{T}(i)}{\partial \theta} \hat{R}^{-1} v(i)\right]_{\theta=\theta_{0}}
\end{aligned}
$$

The output sensitivities quantify the changes in the outputs due to alterations in the parameters, and they are linearly independent and nonzero for a reasonable $\Delta \theta^{51}$.

Accurate values for the unknown parameters may be obtained using the output-error method. Using the second order gradient of the cost function and assuming a given constant $R$, the Fisher information matrix may be simplified to establish the maximum likelihood estimator. This parameter covariance matrix of the maximum likelihood estimator represents the Cramer-Rao 
inequality, which indicates the lower bound for parameter covariance matrix. The Cramer-Rao bounds are a standard means of establishing confidence intervals for parameter estimates in aircraft parameter identification ${ }^{51}$. Figure 13 shows the basic block diagram for the output-error method.

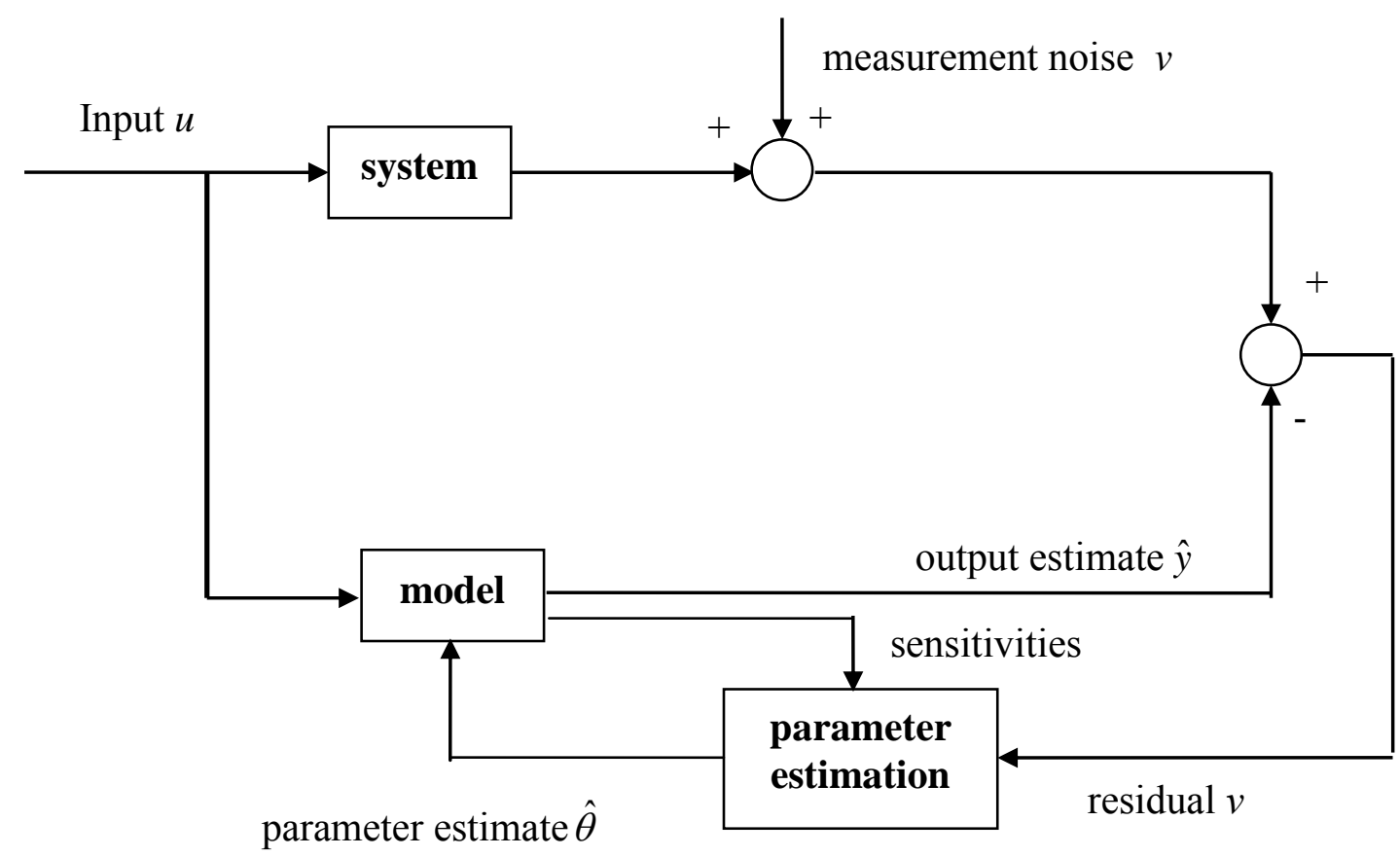

Figure 13: Output-Error Method [Reproduced from Reference 51]

\subsubsection{Parameter Identification in the Frequency Domain - Fourier Transform Regression Method}

One common method used for parameter identification in the frequency domain is from a relatively new class of identification methods and is known as the Fourier Transform Regression (FTR) method. This method is based on the equation error approach, and it is considered "promising and advantageous" when compared to other recursive algorithms ${ }^{65}$. The FTR method utilizes the discrete Fourier transform of the data collected in the time domain using the finite Fourier integral and Euler approximation. Equation 3.13 shows the Fourier transform of an arbitrary signal $x(t)^{65}$.

$$
\tilde{x}(\omega)=\int_{0}^{T} x(t) e^{-j \omega t} d t
$$

With the application of a simple Euler approximation Equation 3.13 becomes: 


$$
\tilde{x}(\omega) \approx \Delta t \sum_{k=0}^{N-1} x_{k} e^{-j \omega k \Delta t}
$$

where $k$ represents the discrete time index, $\Delta t$ represents the sampling interval, and $N$ represents the number of data points. The first-order Euler approximation of the finite Fourier transform of Equation 3.13 is represented by Equation 3.15, which results in the approximate solution shown in Equation 3.16. Equation 3.4 is only valid when using a small sampling time ${ }^{65}$.

$$
\begin{aligned}
\tilde{X}(\omega) & =\sum_{k=0}^{N-1} x_{k} e^{-j \omega k \Delta t} \\
\tilde{x}(\omega) & \approx \tilde{X}(\omega) \Delta t
\end{aligned}
$$

Next, the Fourier transform is applied to a linearized model of the system neglecting the process noise. An equivalent system to the linear model in the time domain is obtained in the frequency domain and is represented generically by Equations 3.17 and 3.18, where the measurement biases and initial conditions have been omitted ${ }^{65}$.

$$
\begin{gathered}
j \omega \tilde{x}(\omega)=A \tilde{x}(\omega)+B \tilde{u}(\omega) \\
\tilde{y}(\omega)=\tilde{x}(\omega)
\end{gathered}
$$

A least squares cost function can be established in the frequency domain for the $k$ th state equation and represented as Equation 3.19, assuming that the measurements of the state, output, and input variables are available ${ }^{65}$.

$$
J_{k}=\frac{1}{2} \sum_{n=1}^{m}\left|j \omega_{n} \tilde{x}_{k}(n)-A_{k} \tilde{x}(n)-B_{k} \tilde{u}(n)\right|^{2}
$$

In Equation 3.19, $m$ represents the number of frequencies and $A_{k}$ and $B_{k}$ represents the $k$ th row of the respective matrices, and $\tilde{x}_{k}(n)$ represents the $k$ th element of vector $\tilde{x}$ for frequency $\omega_{n}$. The number of frequencies $m$ is typically selected between 0.01 to $1.5 \mathrm{~Hz}$ for a rigid body aerodynamic model. For a frequency range of interest, the increments are usually equally spaced between the minimum and maximum frequency values to be evaluated. Additionally, for the application of determining stability and control derivatives for aircraft the zero frequency corresponding to the trim and measurement biases is neglected, which is why it is not necessary to include the initial conditions and measurement biases when converting the linear model to the frequency domain representation - an advantage of the frequency domain approach ${ }^{65}$.

Next, the standard regression problem is formulated as shown in Equation 3.20, 


$$
\tilde{Y}=\tilde{X} \Theta+\tilde{\varepsilon}
$$

where $\tilde{\varepsilon}$ represents the complex equation error in the frequency domain and $\Theta$ represents the vector of unknown model parameters in matrices $A_{k}$ and $B_{k}$. The matrices of independent variables and the vector of dependent variables are represented by Equations 3.21 and 3.22, respectively ${ }^{65}$.

$$
\begin{gathered}
\tilde{X}=\left[\begin{array}{cc}
\tilde{x}^{T}(1) & \tilde{u}^{T}(1) \\
\tilde{x}^{T}(2) & \tilde{u}^{T}(2) \\
\vdots & \vdots \\
\tilde{x}^{T}(m) & \tilde{u}^{T}(m)
\end{array}\right] \\
\tilde{Y}=\left[\begin{array}{c}
j \omega_{1} \tilde{x}_{k}(1) \\
j \omega_{2} \tilde{x}_{k}(2) \\
\vdots \\
j \omega_{m} \tilde{x}_{k}(m)
\end{array}\right]
\end{gathered}
$$

The least square cost function may be represented in the frequency domain as shown in Equation 3.23 .

$$
J=\frac{1}{2}(\tilde{Y}-\tilde{X} \Theta)^{*}(\tilde{Y}-\tilde{X} \Theta)
$$

where "*" indicates the complex conjugate transpose. A minimization of the cost function as described by Reference 65 gives the following representation of the estimates:

$$
\hat{\Theta}=\left[\operatorname{Re}\left(\tilde{X}^{*} \tilde{X}\right)\right]^{-1} \operatorname{Re}\left(\tilde{X}^{*} \tilde{Y}\right)
$$

The standard deviations of these estimates are determined from the square root of the diagonal elements of the parameter error covariance matrix $P$, described by Equation 3.25.

$$
P=E\left[(\hat{\Theta}-\Theta)(\hat{\Theta}-\Theta)^{T}\right]=\operatorname{cov}(\hat{\Theta})=\sigma^{2}\left[\operatorname{Re}\left(\tilde{X}^{*} \tilde{X}\right)\right]^{-1}
$$

where the equation error covariance $\sigma^{2}$ is estimated from the residuals ${ }^{65}$ :

$$
\sigma^{2}=\frac{1}{m-n_{q}}\left[(\tilde{Y}-\tilde{X} \hat{\Theta})^{*}(\tilde{Y}-\tilde{X} \hat{\Theta})\right]
$$

where $n_{q}$ represents the number of unknown parameters.

The process above describes a one step method for obtaining estimates of the unknown parameters; however, it is often desirable to utilize a recursive adaptation of this algorithm. To do this, Equation 3.15 is modified to reflect the discrete Fourier Transform at any discrete time 
point $k$. If we assume that an additional sampling point is available and we separate the last point from the summation, Equation 3.15 becomes $^{65}$ :

$$
\tilde{X}_{k+1}(\omega)=\sum_{i=0}^{k-1} x_{i} e^{-j \omega i \Delta t}+x_{k+1} e^{-j \omega(k+1) \Delta t}=\tilde{X}_{k}(\omega)+\tilde{x}_{k+1} e^{-j \omega(k+1) \Delta t}
$$

where $i$ is used to replace the index $k$ to eliminate confusion. This iterative form of the Fourier Transform Regression method can then be used in the same way as the single point form and implemented at each time step.

The FTR method has several advantages: there are no tuning parameters, the algorithm is relatively simple to implement, and since the zero frequency is omitted the measurement biases are eliminated. However, the elimination of the zero frequency raises the issue of dealing with trim values. Because lumped bias terms do not exist in this format, the trim values must be removed from the measurements as well as the control surfaces before applying the FTR. Another advantage is due to the specific frequency range being selected for this algorithm, which allows the FTR to automatically filter the noise outside the range of interest. The cutoff frequency must be selected in this case, and it must be at least higher than the natural frequency of the short period mode. Because of these advantages leading to reduced computation time, the FTR has a high suitability for real-time parameter estimation and is often implemented in UAV systems for this purpose. There are also some disadvantages of the FTR approach including the assumption that the state variable measurements are available and have no errors. It is also important to note that the FTR is limited to linear models ${ }^{65}$. 


\section{Research Approach and Methodology}

\subsection{WVU YF-22 Research Platform}

The WVU YF-22 research aircraft was one of the platforms used for the validation of faulttolerant flight control laws. Before these control laws could be developed, an accurate mathematical model of the aircraft had to be identified, including the contribution from individual control surfaces on the aircraft dynamics. The following sections describe the WVU YF-22 aircraft platform and its capabilities for flight testing.

\subsubsection{WVU YF-22 Aircraft System}

The YF-22 research aircraft, shown in Figure 14, was designed, constructed, and instrumented by researchers at WVU. The aircraft is an approximate $1 / 8$ semi-scale model of the full size F-22 aircraft. Through a design analysis prior to construction, a perfectly scaled model was found to not have an appropriate wing load parameter resulting in a reduction of the handling quality of the aircraft ${ }^{68}$. The resulting semi-scaled aircraft has a total length of $2.3 \mathrm{~m}$ with a $2.0 \mathrm{~m}$ wingspan; the takeoff weight is approximately $23 \mathrm{~kg}$, including an approximate 5 $\mathrm{kg}$ electronic payload. The payload consists of a PC-104 form factor, customized electronic boards, a complete sensor suite, and a GPS receiver. A Ram 1000 turbine engine provides $125 \mathrm{~N}$ (approximately $28 \mathrm{lbs}$ ) of thrust with a fuel capacity of approximately $3.5 \mathrm{~L}$ of jet $\mathrm{A}$ fuel ${ }^{68}$ for a mission length of approximately 10 minutes.

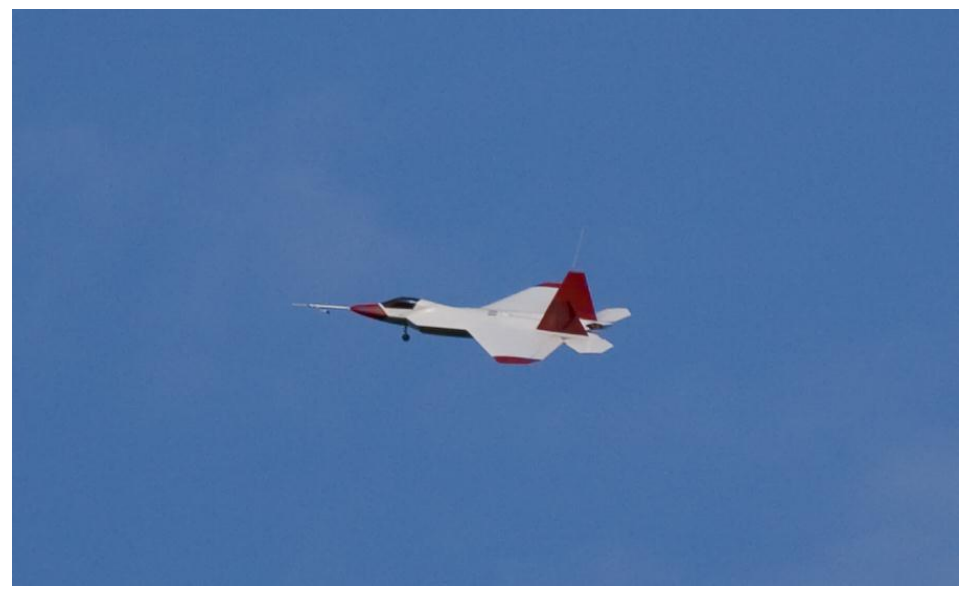

Figure 14: WVU YF-22 Research Aircraft

The primary control surfaces - ailerons, stabilators, flaps, and rudders - are all commanded using digital servos and are instrumented with potentiometers to measure the surface deflections. An additional digital servo is used to activate the pneumatic braking system upon the command 
from the pilot. The jet engine is controlled by an Engine Control Unit (ECU), which monitors the exhaust gas temperature and engine compressor pressure and controls the pump drive voltage ${ }^{68}$. The interested reader is referred to References 68 and 69 for an extensive description of the research aircraft design, construction, and payload.

Special consideration was given to electromagnetic interference (EMI); the design, manufacturing, and installation of the customized and "off-the-shelf" components were completed with the goal of minimizing EMI. Aluminum enclosures were used to shield most of the hardware components, and ferrite RF chokes were inserted along power and signal cables. The payload systems were then evaluated with a spectrum analyzer to assist in addressing EM interference ${ }^{68,69}$.

\subsubsection{WVU YF-22 Onboard Computer}

The YF-22 system is based on a PC-104 computer system, consisting of a CPU module, a Data Acquisition (DAQ) module, and a power supply module interfaced with two customized circuit boards - the controller board and the interface board. A $64 \mathrm{MB}$ compact flash card contains the Linux operating system and flight control laws. The compact flash card is interfaced with an IDE compact flash adapter. The upper part of Figure 15 shows the location of the instrumentation package within the cargo bay while the lower part of Figure 15 shows the internal PC-104 assembly ${ }^{68,70}$.
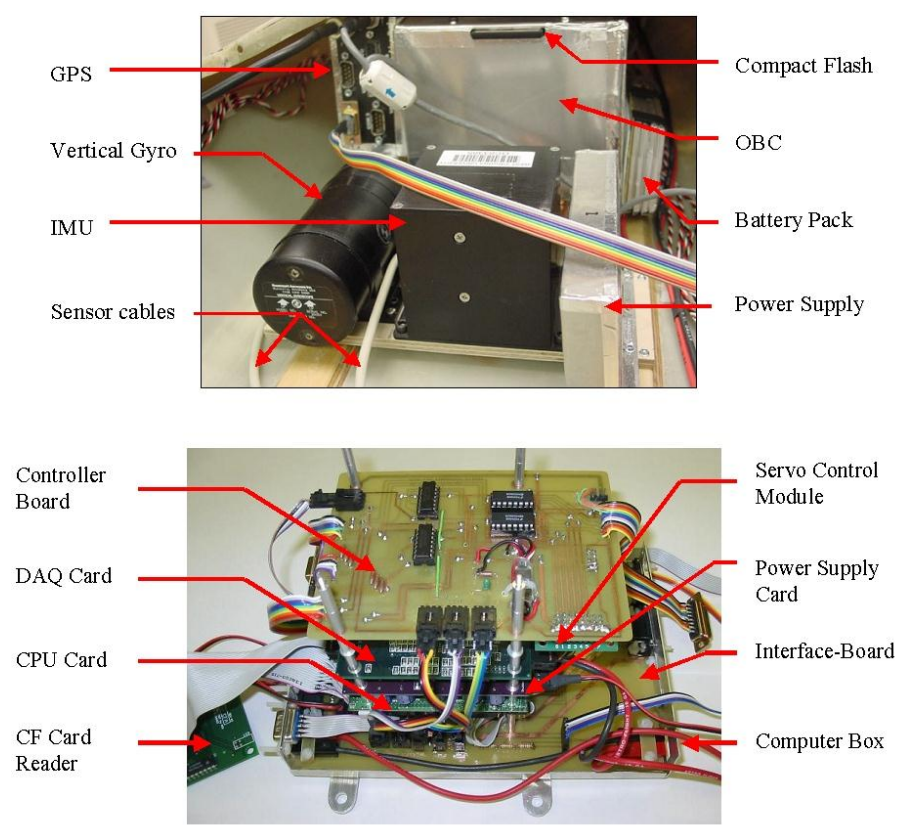

Figure 15: On-Board Instrumentation Package ${ }^{68}$ 
The CPU is a low-power computer (MSI-CM588) with a 6x86 $300 \mathrm{MHz}$ processor. The DAQ card (Diamond-MM-32-AT) features 32 analog input channels with 16-bit resolution and 24 digital I/O channels. The interface board is used for linking individual sensor outputs to a specific data acquisition channel which, in turn, re-routes power from the on-board power supply (Jupiter-MM-SIO) to the sensors. This connection scheme does not include the vertical gyro and the GPS receiver, which are powered through a separate power supply ${ }^{6,69}$.

The customized controller board functionality includes the following:

1. Receiving control signals from the onboard computer (OBC), and translating them into Pulse Width Modulation (PWM) signals;

2. Receiving PWM control signals from the radio receiver;

3. Dispatching the control signals from the $\mathrm{OBC}$ or the radio receiver to the individual servos (according to the current operation mode of the aircraft).

\subsubsection{WVU YF-22 Sensors and Communication Hardware}

The WVU YF-22 aircraft is instrumented with a complete suite of sensors for measuring a variety of flight data parameters. An inertial measurement unit (IMU) provides measurements of the linear accelerations and angular rates, and a mechanical gyroscope provides measurements for the pitch and bank angles. A GPS receiver provides position and velocity information in three dimensions with respect to the earth reference frame, and the air data probe provides measurements of the angle of attack and sideslip angle. It was experimentally evaluated that the noise for all of the sensors could be approximated to follow a Gaussian probability density function. The ,3 $3 \sigma^{\prime}$ values from ground tests for each of the vehicle sensors are provided below $^{68,69,70}$.

- Inertial Measurement Unit (Crossbow IMU400), providing 12-bit measurements for the accelerations $a_{x}, a_{y}, a_{z}$ (range $\pm 4 \mathrm{~g}$, with $3 \sigma=0.06 \mathrm{~g}$ ), and the angular rates $p, q$, and $r$ (range $\pm 90 \%$ sec with $3 \sigma=1 \%$ ).

- Vertical gyro (Goodrich-VG34), providing measurements for the pitch and roll Euler's angles $(\theta$ and $\phi)$ with ranges of $\pm 60^{\circ}$ and $\pm 90^{\circ}$ respectively and $3 \sigma=0.35^{\circ}$.

- GPS receiver (Novatel-OEM4), providing measurements for $x, y, z, V_{x}, V_{y}, V_{z}$ with respect to an earth reference frame, with $3 \sigma=0.7 \mathrm{~m}$ for the positions and $3 \sigma=0.1 \mathrm{~m} / \mathrm{s}$ for the velocities. 
- Potentiometers for the primary control surfaces ( $10 \mathrm{k} \Omega$ each, under a $12 \mathrm{~V}$ supply), providing measurements for $i_{H}, \delta_{A}, \delta_{R}$, with ranges of $[-1,8]^{\circ},[-10,10]^{\circ}$, and $[-7,7]^{\circ}$ and with $3 \sigma=0.6^{\circ}, 0.3^{\circ}$, and $0.15^{\circ}$ respectively.

- Air Data Probe, (SpaceAge ${ }^{\mathbb{O}}$ Inc. Mini Air Data Boom), providing measurements of flow angles $\alpha$ and $\beta$, with ranges of $\pm 30^{\circ}$ and $3 \sigma=0.15^{\circ}$.

- Absolute and Differential pressure sensors (SenSym ASCX15AN and ASCX01DN), with ranges of [0-15] and [0-1] PSI and $3 \sigma=0.06$ and 0.0015 PSI respectively. Both sensors were connected to the nose probe providing measurements for $H$ and $V$.

- Temperature sensor (Thermistor under a $5 \mathrm{~V}$ supply).

\subsubsection{WVU YF-22 Data Acquisition Software}

Software that executes the designed flight control scheme (FCS) was featured on the onboard computer. The operating system was based on a Linux kernel (Version 2.6.9), patched with the Real Time Application Interface (RTAI, Version 3.2), allowing for the execution of the flight control software with strict timing constraints. Due to the constraints of the on-board memory, the RTAI patched kernel was compiled with a minimum amount of features and Busybox ${ }^{\circledR}$ software, which provides the required Linux utilities.

The FCS was designed and implemented using the Matlab/Simulink ${ }^{\circledR}$ environment to perform data acquisition, communication, execution of control laws, and implementation of the on-board computer-generated control commands ${ }^{71}$. This enabled the $\mathrm{OBC}$ to collect and store information from the aircraft sensors during the flight test, respond to pilot commands, and utilize autonomous capabilities, all of which were integral components for performing this parameter identification study.

\subsection{WVU YF-22 Flight Test Design}

The first vital component to a successful parameter identification study is an appropriate experiment design. In the case of a parameter identification study with WVU YF-22, it was necessary to derive a nominal mathematical model of the aircraft, that is, all control surfaces

performing as expected. Additionally, a mathematical model of the aircraft reflecting the individual control surface effects on the dynamic behavior of the aircraft was identified for application within a fault-tolerant flight control system and the handling of actuator failures. In order to accomplish this, individual control surface maneuvers were designed and performed in 
flight to model these effects. Specifically, individual control surface deflections caused a crosscoupling between the longitudinal and lateral-directional dynamics that was modeled so that they could be accounted for within the flight control system design. For this parameter identification study, "failures" were injected onto specified control surfaces via the onboard computer during a maneuver. Failures were only implemented onto the ailerons and stabilators, as the rudders were commanded off of a single input channel on the WVU YF-22.

In order to successfully identify a mathematical model of the WVU YF-22 under both nominal and failure conditions for implementation into a fault-tolerant FCS, the flight tests were designed to collect maximum data content, i.e. several maneuvers per lap. Since the aircraft has the capability of autonomous flight, tests were divided into two phases: pilot-induced and computer-induced PID maneuvers. The following sections detail the flight test experiment designs, which incorporated typical parameter identification maneuvers to excite the aircraft dynamics from which the mathematical model of the aircraft was derived.

\subsubsection{Remotely-Piloted Flight Test Design}

The first step of the parameter identification process was to have a pilot remotely control the aircraft and perform maneuvers to excite the longitudinal and lateral-directional aircraft dynamics so that a linear model could be extracted from the system response. For the pilotinduced maneuvers, stabilator doublets and 1-1-2-3 maneuvers were injected to excite the longitudinal short period dynamics, while aileron and rudder/aileron doublet combinations were injected to excite the lateral-directional dynamics. Rudder/aileron doublet combinations were used to excite the Dutch roll mode prior to the roll mode due to the slower response of the Dutch roll. This combination of maneuvers allowed for more data to be collected in a single flight since the roll and Dutch roll modes were excited within the combination maneuver.

The pilot-induced maneuvers consisted of both nominal doublets and doublets performed during a surface "failure". Failures were induced by the on-board computer with the pilot-in-theloop injecting the doublet maneuvers on the primary control surfaces. During stabilator doublets, the left stabilator was locked at the trim position, allowing only for the deflection of the right stabilator. Similarly, the aileron and rudder/aileron combination doublets were performed with the left aileron locked at the trim position. Maneuvers performed for this segment of flight testing are summarized in Table 2. 
Table 2: Planned Pilot-Induced Maneuver Summary

\begin{tabular}{|c|c|c|}
\hline Maneuver & Failure & Dynamic Mode excited \\
\hline Stabilator Doublet & No & Short Period \\
\hline Stabilator 1-1-2-3 Maneuver & No & Short Period \\
\hline Aileron Doublet & No & Roll \\
\hline Rudder/Aileron Doublet Combination & No & Dutch Roll \& Roll \\
\hline Stabilator Doublet & Left Stabilator & Short Period \& Roll Behavior \\
\hline Aileron Doublet & Left Aileron & Roll \& Pitching Behavior \\
\hline Rudder/Aileron Doublet Combination & Left Aileron & Dutch Roll, Roll, \& Pitching Behavior \\
\hline
\end{tabular}

\subsubsection{On-Board Excitation System Development and Autonomous Flight Test Design}

The WVU YF-22 is a remotely-piloted aircraft; however, it has the ability to fly autonomously. For this specific set of flights, an On-Board Excitation System (OBES) ${ }^{72,73}$ was developed in coordination with a "Virtual Leader" (VL) scheme that was originally employed towards the goals related to a formation flight demonstration ${ }^{68,69}$. The previously utilized formation flight VL scheme allowed for detailed testing of the formation control laws prior to flying an actual 2-aircraft configuration. The original experiment consisted of a single aircraft tracking a 3-D trajectory for a VL, as illustrated in 2-D by Figure 16, which was essentially a flight path previously recorded by one of the aircraft.

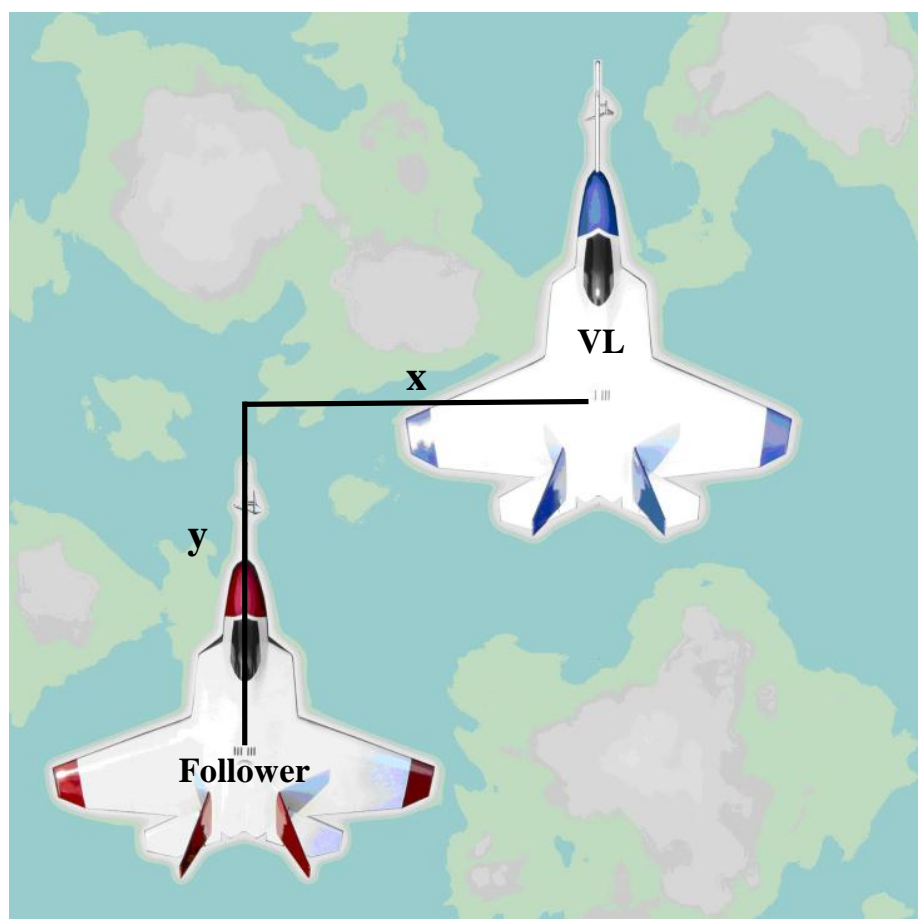

Figure 16: Virtual Leader (Blue), Simulated Follower (Red) 
The actual aircraft follows at a specified position behind the VL trajectory, which was loaded into the on-board computer ${ }^{68}$. This VL methodology was implemented on the VL scheme designed for the PID flight tests. The modified VL scheme consisted of an artificial GPS track and aircraft angular orientation sent to the on-board controller. The track provided the GPS position and velocity information to the "follower" aircraft when systems were switched into autonomous mode. In this configuration, the "follower" aircraft mimicked the position of the VL, instead of following at a specified distance behind. PID maneuvers were embedded in this autonomous flight, and had to be performed at predefined points in the GPS track. An OBES was used to perform the maneuvers at points in the GPS trajectory where there was enough time and space for the aircraft to complete doublet maneuvers to excite the aircraft dynamics and allow for the response to dampen. Thus, the Virtual Leader path was designed to consist of two $650 \mathrm{~m}$ straight legs in parallel to the runway at the Louis-Bennett Airfield at WVU Jackson's Mill to allow for doublet maneuver injection and sufficient time for the aircraft dynamic response to be measured. Two semicircular turns were implemented into the VL path for a smooth transition to set up for the next maneuver. Figure 17 shows the Virtual Leader path for the autonomous PID flights.

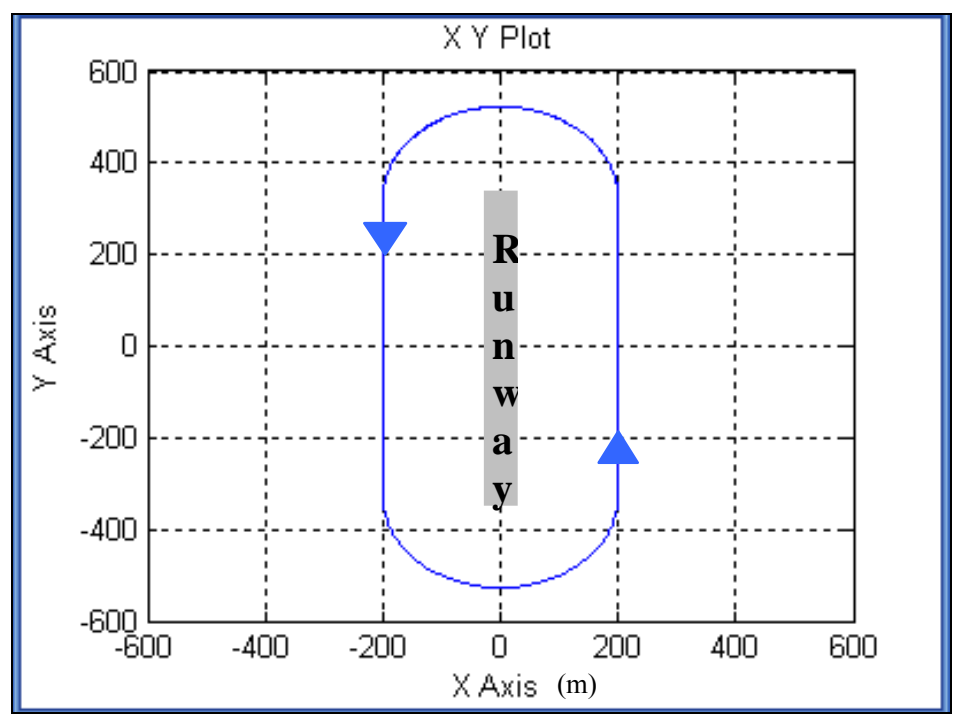

Figure 17: Projected Virtual Leader Flight Path for PID Study (in meters)

The OBES was designed to inject doublet maneuvers on a designated pair of control surfaces at specific points in the flight trajectory similar to those injected by the pilot. To accomplish this, the OBES was coordinated with the Virtual Leader track. At a specific position in the VL path 
once the aircraft completed the turn, the GPS tracking was turned "off" so that the aircraft no longer mimicked the coordinates and behavior of the VL. At this point, the control surfaces ailerons, stabilators, and rudders - were set to their trim positions at approximately 0.2 seconds prior to the injection of the maneuver. This was done to ensure the aircraft was in steady level flight prior to the injection of the doublet as to not have an influence on the dynamic response by a pre-existing response to stray surface movement. Once the desired doublet maneuver was injected, a 5.0 to 7.0 second period was allotted so that the aircraft dynamic response could be measured and recorded by the sensors and onboard computer, respectively, free of a command input. Immediately following the 5.0 to 7.0 second period, the GPS tracking resumed and the aircraft set up for another maneuver.

The OBES is an attractive feature for flight testing purposes due to the repeatability of the computer system injecting identical maneuvers during the experiments. Initial flights were conducted with the OBES software injecting stabilator, aileron, and rudder/aileron combination doublets separately on the "healthy" aircraft with the goal of exciting the longitudinal and lateraldirectional dynamics separately. When designing the OBES maneuvers, the doublet amplitudes and durations were designed to be similar to those injected manually by the pilot. The lateraldirectional dynamics - specifically the period of the Dutch roll - as observed from previously measured flight data, was used for programming the OBES rudder/aileron combination maneuver. Sequencing the rudder and aileron doublets near the period of the Dutch roll mode is aimed at producing the best PID maneuver; therefore, the Dutch roll mode responses from the previous pilot-injected doublets were evaluated to optimize the maneuver design for the OBES inputs.

In order to model the individual control surface effects on the aircraft dynamics, a locked actuator was simulated during the computer-injected doublets as they were during the pilot-inthe-loop scenario. In the autonomous flight configuration, a single control surface was locked while the OBES injected a doublet maneuver on the other corresponding surface. The doublet maneuvers performed during the manually-piloted flights were repeated in autonomous mode. Failures on the rudder surfaces were not evaluated during these experiments since the WVU YF22 did not have the capability of individual rudder commands. 


\subsection{WVU YF-22 State Space Parameter Identification}

In order to design a control system for the WVU YF-22, an accurate linear model of the aircraft had to be identified. The goal of this study was to derive a complete state-space linear representation of the WVU YF-22 under both nominal and actuator failure conditions that may be utilized in the development of fault-tolerant flight control laws. Figure 18 highlights the goals of the parameter identification study, including each type of model requiring identification.

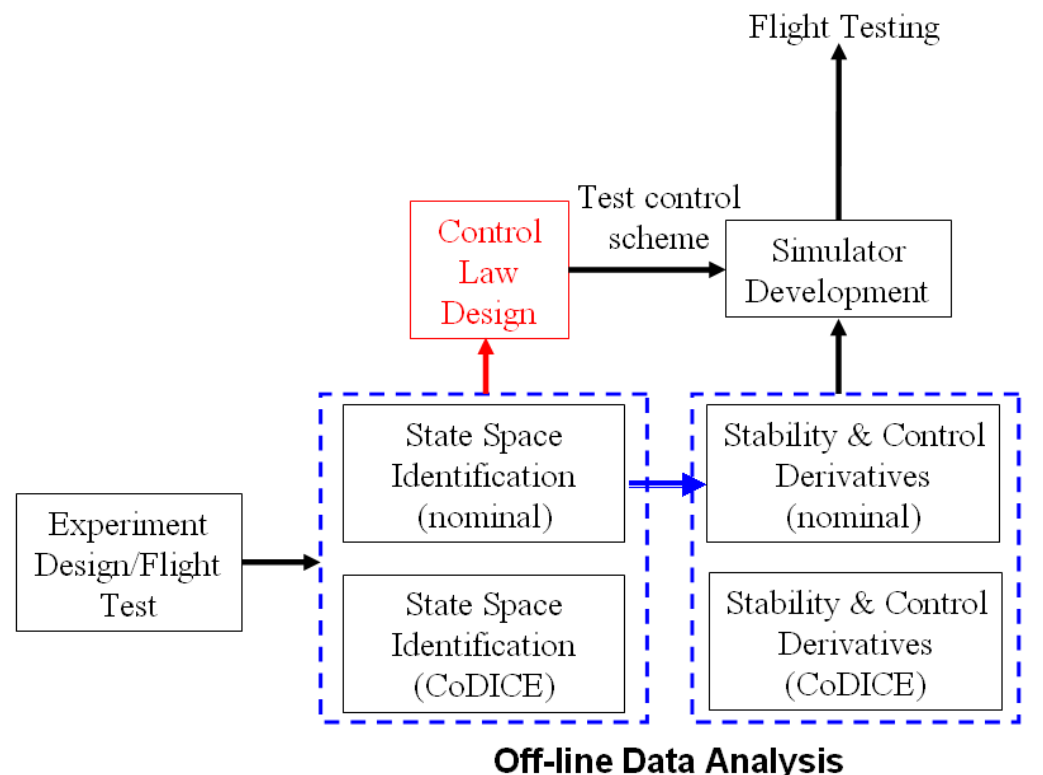

Figure 18: Overview of Aircraft Parameter Identification Process for WVU YF-22

The Matlab ${ }^{\circledR}$ System Identification Toolbox was utilized for the estimation of an initial representation of the linear aircraft model in the time domain under nominal conditions. This estimate was then utilized as a starting point for an optimization scheme to minimize the error between the measured data and model outputs for selected states.

\subsubsection{Matlab $^{\circledR}$ System Identification Toolbox}

Preliminary linear parameter identification was conducted using the Matlab ${ }^{\circledR}$ System Identification (SysID) Toolbox. The SysID Toolbox allows for the estimation of linear and nonlinear mathematical models of dynamic systems from measured data ${ }^{74}$. Using this toolbox, the collected data was pre-processed for parameter identification by removing the means from the measured signals or running the data through a filter. The toolbox allowed for the data to be 
quickly run through several different model types for quick evaluation of the model performance. Figure 19 shows the System Identification Toolbox main screen.

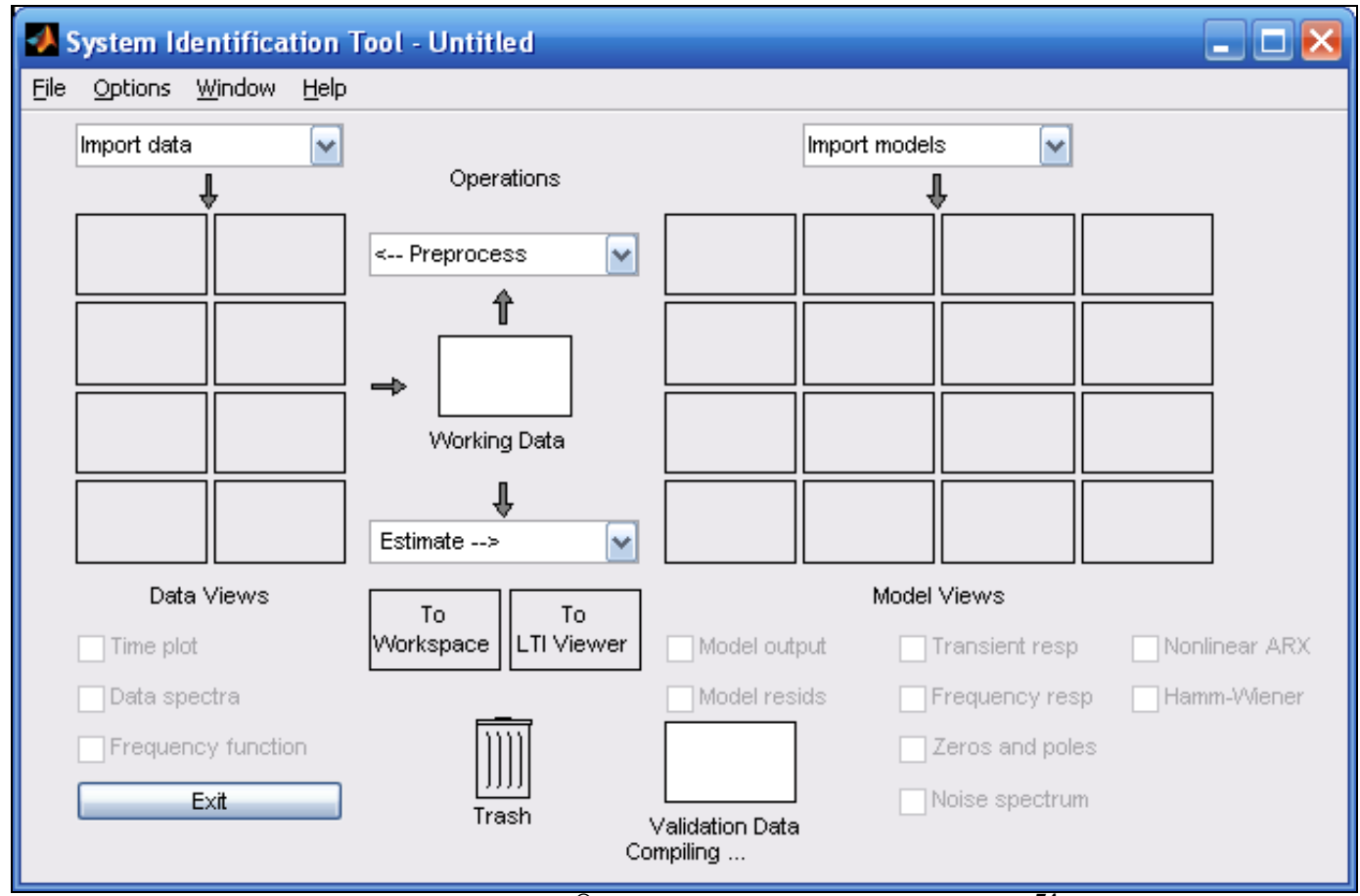

Figure 19: Matlab ${ }^{\circledR}$ System Identification Toolbox ${ }^{74}$

With this tool, segmented flight data was imported into the toolbox. Once the working data segment was selected, the order of the model was chosen based on the number of outputs being measured. The System Identification Toolbox has several different models available to the user, some of which are described in the Section 4.3.2. Once the model was selected, the data was run through the model and posted in the Model Views section. The user has several different options to view and assess the validity of the model. Model Output allows the user to visually compare the simulated output of the channels to the measured data. The System Identification Toolbox also provides a measure of accuracy between 0 to 100 to give the user a quantitative measure of how the model is performing. If several sets of similar data were loaded into the toolbox, the sets not used for identification may be utilized for validation. This is done by the user selecting a validation set of data and selecting the Model Output. The toolbox provides a measure of accuracy of the other data set to the identified model. The model information output is a state space representation for which the eigenvalues and eigenvectors are used to assess the stability of the modeled system. 
Parameter identification is generally an iterative process, and this toolbox makes it easy for many models to be evaluated from given sets of data in a relatively short amount of time. Data may be imported into the toolbox in either the time or frequency domains, and following the estimation of a model they may be evaluated using the built-in functions of the toolbox. Due to its ease of use as well as success in past efforts ${ }^{68}$, the Matlab ${ }^{\circledR}$ System Identification Toolbox was used to estimate a preliminary model to serve as a starting point for optimization.

\subsubsection{Nominal Linear Parameter Identification - State Space Representation}

Nominal linear parameter identification was divided into two segments: longitudinal and lateral-directional state space model identification. The Matlab ${ }^{\circledR}$ System Identification Toolbox was used to independently identify the longitudinal and lateral-directional linear aircraft models from nominal doublet maneuvers performed during flight testing. The following sections provide specific detail on the inputs and output states that were used in the identification process as well as the proposed estimator from the Matlab ${ }^{\circledR}$ System Identification Toolbox.

\subsubsection{Longitudinal State Space Parameter Identification}

For the longitudinal linear model identification, the data sets with nominal stabilator doublets were utilized with the System Identification Toolbox. First, the data was segmented based on the doublet maneuvers performed in flight tests. A doublet maneuver and its dynamic response produced one segment of flight data that was used in the toolbox. There were a large set of maneuvers from the collected flight data so that different segments could be used for validation once a model was identified. The stabilator doublet was set as the system input, while the angle of attack $\alpha$ and the pitch rate $q$ were set as the output states, as reflected by Equation 4.1. Since two output states were considered, a second-order linear-parametric model was used.

$$
\left[\begin{array}{c}
\dot{\alpha} \\
\dot{q}
\end{array}\right]=A_{L G_{(22)}}\left[\begin{array}{c}
\alpha \\
q
\end{array}\right]+B_{L G_{(221)}} i_{H}
$$

A previous study estimating the nominal longitudinal linear model of the WVU YF-22 from flight data successfully utilized the "N4SID" algorithm. The N4SID algorithm addresses the problem of identifying the parameters of an $m$-input, $l$-output linear, time-invariant $n^{\text {th }}$ order state space system. This model assumes that the inputs are noise-free and the outputs may contain noise ${ }^{75}$. N4SID produces an identified model in the following form where: 


$$
\begin{aligned}
& x_{k+1}=A x_{k}+B u_{k}+w_{k} \\
& \tilde{y}_{k}=C x_{k}+D u_{k}+v_{k}
\end{aligned}
$$

With N4SID, a priori knowledge of the order of the observability or controllability is not required. This is because the state space matrices are calculated as full state space matrices and not in their canonical forms. One other main advantage of the N4SID algorithms is that they are non-iterative ${ }^{76}$. The application of the N4SID algorithm in this case produced the longitudinal state space matrices that were used as the initial point for optimization.

In order to derive the longitudinal linear model, the segmented flight data was imported into the Matlab ${ }^{\circledR}$ System Identification Toolbox in the time domain. Once the data was preprocessed (means removed from segments), it was used for model identification with the N4SID algorithm. Once an adequate model was output from the algorithm, it was validated through the toolbox using other flight data segments containing doublet maneuvers. Once the model was validated, the state matrix $A$ was used to verify the stability of the model through an analysis of the eigenvalues. These eigenvalues represents the short period response of the aircraft and from these values the damping and natural frequency of the short period response was calculated. There was not sufficient time following the maneuvers to collect enough data to accurately identify the phugoid response of the aircraft. However, the $\dot{V}$ equation was estimated through direct measurement based off of Equation 4.3, which represents the decoupled longitudinal force equation for $\dot{V}$.

$$
m \dot{V}_{T}=F_{T} \cos \left(\alpha+\alpha_{T}\right)-D-m g_{D} \sin (\theta-\alpha)
$$

The final form of the longitudinal linear model is shown in Equation 4.4.

$$
\left[\begin{array}{c}
\dot{V} \\
\dot{\alpha} \\
\dot{q} \\
\dot{\theta}
\end{array}\right]=A_{L G_{(4 \times 4)}}\left[\begin{array}{c}
V \\
\alpha \\
q \\
\theta
\end{array}\right]+B_{L G_{(4 \times 1)}} i_{H}
$$

\subsubsection{Lateral-Directional State Space Parameter Identification}

For the lateral-directional linear model identification, data sets with nominal aileron and rudder/aileron combination doublets were utilized with the System Identification Toolbox. First, the data was segmented based on the doublet maneuvers performed in flight tests. A doublet maneuver and its dynamic response produced one segment of flight data that was used in the 
toolbox. The aileron and rudder channels were set as the system inputs, while the three state outputs were the sideslip $\beta$, the roll rate $p$, and the yaw rate $r$, as reflected by Equation 4.5. Since three output states were considered, a third-order linear-parametric model was used.

$$
\left[\begin{array}{c}
\dot{\beta} \\
\dot{p} \\
\dot{r}
\end{array}\right]=A_{L T_{(3 \times 3)}}\left[\begin{array}{c}
\beta \\
p \\
r
\end{array}\right]+B_{L T_{(3 \times 2)}}\left[\begin{array}{l}
\delta_{A} \\
\delta_{R}
\end{array}\right]
$$

A previous study estimating the nominal lateral-directional linear model of the WVU YF-22 from flight data successfully utilized the prediction-error minimization or "PEM" algorithm ${ }^{68,77}$. The prediction-error minimization algorithm is a method based on estimating the parameters of a linear model by minimizing a robustified quadratic prediction error criterion with an iterative search algorithm ${ }^{74}$. With this algorithm, an $n^{\text {th }}$ order state space model is estimated in the following form:

$$
\begin{aligned}
& x(t+T s)=A x(t)+B u(t)+K e(t) \\
& y(t)=C x(t)+D u(t)+e(t)
\end{aligned}
$$

where $t$ is the current time step and $T s$ is the sampling time.

The basis for the PEM algorithm is to estimate the model parameters which minimize the optimally determined one-step-ahead output prediction error. The PEM attempts to find a parameter that minimizes the prediction error and if the model has a different structure from the process, the parameter is calculated so that the prediction error is minimized under its structural constraints $^{78}$, typically leading to an unbiased estimate.

In order to derive the lateral-directional linear model, the segmented flight data was imported into the Matlab $^{\circledR}$ System Identification Toolbox in the time domain. Once the data was preprocessed, it was used for model identification with the PEM algorithm. Once an adequate model was output from the algorithm, it was validated through the toolbox using other flight data segments containing similar doublet maneuvers. The Dutch roll response was estimated from rudder doublets and rudder/aileron doublet combination maneuvers. During aileron maneuvers, only the roll mode was excited so those doublets were only used for roll behavior identification. The rudder/aileron doublet combinations excited both the Dutch roll and roll modes, so one of these maneuvers could be used for complete lateral-directional identification. The aileron doublet maneuvers were used as an additional validation of the roll response obtained from the combination maneuvers. 
Once the model was validated, the state matrix $A$ was used to verify the stability of the model through an analysis of the eigenvalues. These eigenvalues represent the Dutch roll and roll responses of the aircraft. From the eigenvalues, the damping and natural frequency of the Dutch roll response and the roll time constant were also calculated. The final representation of the lateral-directional linear model is shown in Equation 4.7.

$$
\left[\begin{array}{c}
\dot{\beta} \\
\dot{p} \\
\dot{r} \\
\dot{\phi}
\end{array}\right]=A_{L T_{(4 \times 4)}}\left[\begin{array}{c}
\beta \\
p \\
r \\
\phi
\end{array}\right]+B_{L T_{(4 \times 2)}}\left[\begin{array}{c}
\delta_{A} \\
\delta_{R}
\end{array}\right]
$$

\subsubsection{CoDICE Linear Parameter Identification - State Space Representation}

For the development of the Coupled Dynamics with Individual Control Effectors (CoDICE) linear mathematical model of the aircraft, the three primary control surface pairs were divided into their left and right components, providing a total of six individual surfaces. For this effort, a decoupled linear identification effort was conducted using flight data with "failed" control surfaces. The resulting state matrix $A$ is essentially a combination of the nominal longitudinal and lateral-directional state matrices, including the velocity, angle of attack, angle of sideslip, roll rate, pitch rate, yaw rate, pitch angle, and bank angle components and is considered to remain unchanged for this class of failures, since damage to the aircraft surfaces were not considered. The input matrix $B$, however, accounts for the decoupled control surfaces by incorporating the six inputs individually. The input matrix of the CoDICE linear model was essentially derived by dividing the combined stabilator, aileron, and rudder nominal input matrix into the six individual components, thus halving the numeric values for each of the pair when reassigned to the individual surfaces.

The modeling procedure described above does not account for some components of the CoDICE input matrix at "failure" conditions. These components include the individual stabilator effects on the lateral-directional states and the individual aileron and rudder effects on the longitudinal states. In this case, the individual stabilator inputs have an effect on the angle of sideslip, roll rate, and yaw rate, and the individual aileron inputs have an effect on the angle of attack and pitch rate - which is not observed under nominal conditions. Again for this study, rudder failures were not incorporated, so their contributions to the longitudinal dynamics were not accounted for. 
A full state matrix ( 8 states) was derived by combining the longitudinal and lateraldirectional nominal linear models, and the input matrix was derived by modeling the contributions of each individual control surface on the states. As stated above, the nominal input matrix contributions were split into their respective left and right contributions, but the "unknown" components of the input matrix - the coupled dynamic terms - required identification. These components were identified using flight data with control surface failures paired with a Simulink ${ }^{\circledR}$ scheme designed to use Fourier Transform Regression ${ }^{17,19,51,52,79,80,81}$. This Simulink ${ }^{\circledR}$ scheme was developed using the Parameter Identification Library developed at $\mathrm{WVU}^{82}$ to evaluate sections of flight data where a control surface failure occurs and identify the unknown input matrix components, using the FTR method. The FTR block was designed to solve Equation 4.8 where $E$ and $F$ are known constant vectors and $\Theta$ is an unknown vector to be estimated:

$$
E \dot{z}(t)+F z(t)=x(t)^{T} \Theta
$$

By sampling and applying the Discrete Time Fourier Transform (DTFT) to the input and motion variables at time $t=i \Delta t$ :

$$
j \omega E \tilde{z}(\omega)+F \tilde{z}(\omega)=\tilde{x}(\omega)^{T} \Theta
$$

where

$$
\tilde{x}(\omega)=\sum_{i=0}^{N-1} x(i \Delta t) e^{-j \omega i \Delta t}, \tilde{z}(\omega)=\sum_{i=0}^{N-1} z(i \Delta t) e^{-j \omega i \Delta t}
$$

In the case of a failed stabilator, where the angle of sideslip, roll rate, and yaw rate due to the individual stabilator contributions require identification, $x$ in Equation 4.8 represents the deflection of the healthy individual surface, and $F$ represents the affected states mentioned. In the case of a failed aileron affecting angle of attack and pitch rate, $x$ represents the deflection of the healthy individual surface, and $F$ represents those affected states mentioned. During the identification process, the "unknown" input matrix contributions were determined using data from "failure" flights. In these trials, the components of the output vector $\Theta$ represent the unknown values within the input matrix for that particular control surface deflection. The behavior of the aircraft during the failure flight scenarios was used to identify the unknown values in the CoDICE linear model input matrix.

The "unknown" contributions were identified using only the right stabilator or right aileron contributions; however, when identifying the final input matrix of the CoDICE linear model, the 
right control surface contribution was replicated for that of the left control surface with special attention placed on the sign conventions.

The sign convention for the decoupled control surfaces is shown in Equations 4.11-4.13. These equations represent the combination of the individual left and right control surface components and how they equate to the total contribution from the surface pair under nominal conditions.

$$
\begin{gathered}
\mathrm{i}_{\mathrm{H}}=\frac{1}{2}\left(\mathrm{i}_{\mathrm{H}_{\mathrm{L}}}+\mathrm{i}_{\mathrm{H}_{\mathrm{R}}}\right) \\
\delta_{\mathrm{A}}=\frac{1}{2}\left(\delta_{\mathrm{A}_{\mathrm{R}}}-\delta_{\mathrm{A}_{\mathrm{L}}}\right) \\
\delta_{\mathrm{R}}=\frac{1}{2}\left(\delta_{\mathrm{R}_{\mathrm{L}}}+\delta_{\mathrm{R}_{\mathrm{R}}}\right)
\end{gathered}
$$

Based on these equations, in the case of the left and right stabilator affecting the roll rate, the signs of the input matrix contributions are opposite, mimicking the aileron sign convention. In the case where the left and right ailerons affect the pitch rate, the signs of the input matrix contributions are the same, mimicking the stabilator sign convention. Using these conventions for the input matrix, the resulting CoDICE linear mathematical model for the WVU YF-22 is shown in Equation 4.14.

$$
\left[\begin{array}{c}
\dot{V} \\
\dot{\alpha} \\
\dot{q} \\
\dot{\theta} \\
\dot{\beta} \\
\dot{p} \\
\dot{r} \\
\dot{\phi}
\end{array}\right]=A_{C_{\left.C o D I C E_{(88)}\right)}}\left[\begin{array}{c}
V \\
\alpha \\
q \\
\theta \\
\beta \\
p \\
r \\
\phi
\end{array}\right]+B_{C D I C E_{(886)}}\left[\begin{array}{l}
i_{H_{L}} \\
i_{H_{R}} \\
\delta_{a_{L}} \\
\delta_{a_{R}} \\
\delta_{r_{L}} \\
\delta_{r_{R}}
\end{array}\right]
$$

\subsection{WVU YF-22 Stability and Control Derivative Identification}

While the nominal and CoDICE linear models will be utilized for control design purposes, the stability and control derivatives representing the nominal and CoDICE models are necessary for the development of a detailed simulator for fault-tolerant control law validation purposes. The following sections describe the identification and optimization of these stability and control derivatives for the WVU YF-22. 


\subsubsection{Nominal Stability and Control Derivative Modeling}

The mathematical model of an aircraft is described by a generalized set of nonlinear differential equations, shown in Equations 4.15 through $4.24^{81,83,84,85}$. The force equations are represented in Equations 4.15 - 4.17 and the moment equations are represented in Equation 4.18. These equations are representative of the aircraft model based on the body-axis coordinate system and assume that the thrust is aligned with the longitudinal axis of the aircraft.

$$
\begin{aligned}
& \dot{V}=\frac{1}{m}\left(-\frac{1}{2} \rho S V^{2} C_{D} \cos \beta+\frac{1}{2} \rho S V^{2} C_{Y} \sin \beta+T \cos \alpha \cos \beta\right) \\
& -g(\sin \theta \cos \alpha \cos \beta-\cos \theta \sin \phi \sin \beta-\cos \theta \cos \phi \sin \alpha \cos \beta) \\
& \dot{\alpha}=\frac{1}{m V \cos \beta}\left[-\frac{1}{2} \rho S V^{2} C_{L}-T \sin \alpha+m g(\cos \theta \cos \phi \cos \alpha+\sin \theta \sin \alpha)\right] \\
& +q-(p \cos \alpha+r \sin \alpha) \tan \beta \\
& \dot{\beta}=\frac{1}{m V}\left[\frac{1}{2} \rho S V^{2} C_{D} \sin \beta+\frac{1}{2} \rho S V^{2} C_{Y} \cos \beta-T \cos \alpha \sin \beta\right. \\
& +m g(\sin \theta \cos \alpha \sin \beta+\cos \theta \sin \phi \cos \beta-\cos \theta \cos \phi \sin \alpha \sin \beta)]+p \sin \alpha-r \cos \alpha \\
& {\left[\begin{array}{c}
\dot{p} \\
\dot{q} \\
\dot{r}
\end{array}\right]=M_{1}\left[\begin{array}{l}
p^{2} \\
q^{2} \\
r^{2}
\end{array}\right]+M_{2}\left[\begin{array}{l}
q r \\
p r \\
p q
\end{array}\right]+\bar{q} S M_{0}\left[\begin{array}{l}
b C_{l} \\
\bar{c} C_{m} \\
b C_{n}
\end{array}\right]} \\
& \dot{\theta}=q \cos \phi-r \sin \phi \\
& \dot{\psi}=q \sin \phi \sec \theta+r \cos \phi \sec \theta \\
& \dot{\phi}=p+q \sin \phi \tan \theta+r \cos \phi \tan \theta \\
& \dot{x}=V[\cos \beta \cos \alpha \cos \theta \cos \psi+\sin \beta(\sin \phi \sin \theta \cos \psi-\cos \phi \sin \psi) \\
& +\cos \beta \sin \alpha(\cos \phi \sin \theta \cos \psi+\sin \phi \sin \psi)] \\
& \dot{y}=V[\cos \beta \cos \alpha \cos \theta \sin \psi+\sin \beta(\sin \phi \sin \theta \sin \psi+\cos \phi \cos \psi) \\
& +\cos \beta \sin \alpha(\cos \phi \sin \theta \sin \psi-\sin \phi \cos \psi)] \\
& \dot{h}=V(\cos \beta \cos \alpha \sin \theta-\sin \beta \sin \phi \cos \theta-\cos \beta \sin \alpha \cos \phi \cos \theta)
\end{aligned}
$$

The matrices $M_{0}, M_{1}$, and $M_{2}$ are defined as the following: 


$$
\begin{aligned}
& M_{0}=\frac{1}{\operatorname{det}(I)}\left\lfloor\begin{array}{lll}
I_{y y} I_{z z}-J_{y z} J_{y z} & J_{x y} I_{z z}+J_{y z} J_{x z} & J_{x y} J_{y z}+I_{y y} J_{x z} \\
J_{x y} I_{z z}+J_{y z} J_{x z} & I_{x x} I_{z z}-J_{x z} J_{x z} & J_{y z} I_{x x}+J_{x y} J_{x z} \\
J_{x y} J_{y z}+I_{y y} J_{x z} & J_{y z} I_{x x}+J_{x y} J_{x z} & I_{x x} I_{y y}-J_{x y} J_{x y}
\end{array}\right\rfloor \\
& M_{1}=M_{0}\left[\begin{array}{ccc}
0 & J_{y z} & -J_{y z} \\
-J_{x z} & 0 & J_{x z} \\
J_{x y} & -J_{x y} & 0
\end{array}\right] \\
& M_{2}=M_{0}\left[\begin{array}{ccc}
I_{y y}-I_{z z} & -J_{x y} & J_{x z} \\
J_{x y} & I_{z z}-I_{x x} & -J_{y z} \\
-J_{x z} & J_{y z} & I_{x x}-I_{y y}
\end{array}\right]
\end{aligned}
$$

with $I$ being the inertia matrix of the aircraft defined as:

$$
I=\left\lfloor\begin{array}{ccc}
I_{x} & -J_{x y} & -J_{x z} \\
-J_{x y} & I_{y} & -J_{y z} \\
-J_{x z} & -J_{y z} & I_{z}
\end{array}\right\rfloor
$$

The variables $C_{D}, C_{Y}, C_{L}, C_{l}, C_{m}, C_{n}$ are the aerodynamic coefficients, which are used for representing the aerodynamic forces and moments acting upon the aircraft. These coefficients are functions of the aircraft state vector $\left(\xi=[V, \alpha, \beta, p, q, r, \phi, \theta, \varphi, x, y, z]^{\mathrm{T}}\right)$ and input vector $(\delta=$ $\left.\left[\delta_{T}, \mathrm{i}_{\mathrm{H}}, \delta_{A}, \delta_{R}\right]^{\mathrm{T}}\right)$. The aerodynamic coefficients can be approximated by affine functions of the state and input vectors ${ }^{83,84,86}$. Specifically, the aerodynamic coefficients are defined as follows:

$$
\begin{gathered}
C_{D}(\xi, \delta)=c_{D 0}+c_{D \alpha} \alpha+c_{D q} \frac{\bar{c}}{2 V} q+c_{D_{l H}} i_{H} \\
C_{L}(\xi, \delta)=c_{L 0}+c_{L \alpha} \alpha+c_{L q} \frac{\bar{c}}{2 V} q+c_{L_{H H}} i_{H} \\
C_{m}(\xi, \delta)=c_{m 0}+c_{m \alpha} \alpha+c_{m q} \frac{\bar{c}}{2 V} q+c_{m_{i H}} i_{H} \\
C_{Y}(\xi, \delta)=c_{Y 0}+c_{Y \beta} \beta+c_{Y p} \frac{b}{2 V} p+c_{Y r} \frac{b}{2 V} r+c_{Y \delta_{A}} \delta_{A}+c_{Y \delta_{R}} \delta_{R} \\
C_{l}(\xi, \delta)=c_{l 0}+c_{l \beta} \beta+c_{l p} \frac{b}{2 V} p+c_{l r} \frac{b}{2 V} r+c_{l \delta_{A}} \delta_{A}+c_{l \delta_{R}} \delta_{R} \\
C_{n}(\xi, \delta)=c_{n 0}+c_{n \beta} \beta+c_{n p} \frac{b}{2 V} p+c_{n r} \frac{b}{2 V} r+c_{n \delta_{A}} \delta_{A}+c_{n \delta_{R}} \delta_{R}
\end{gathered}
$$

where the individual coefficients contributing to the aerodynamic coefficients are referred to as "stability and control derivatives ${ }^{69}$ ". Equations 4.29 through 4.34 represent the total aircraft drag, 
lift, pitching moment, aerodynamic side-force, rolling moment, and yawing moment coefficients, respectively ${ }^{87}$.

The aircraft aerodynamic derivatives were determined by converting the identified nominal state space models to provide a set of initial stability and control derivatives. These derivatives were the initial values that were fed into an optimization scheme to minimize the error between the output states of the simulated aircraft model and an actual set of flight data. The final optimized model was found by adjusting the stability and control derivatives iteratively to minimize the error between the simulated and measured states. The relationships for determining the coefficients of the matrices in the state space model starting from the values of the aerodynamic derivatives and geometric-inertial parameters are well known ${ }^{83}$. By inverting these relationships ${ }^{69}$, and by using the values of the experimentally determined geometric and inertial parameters, it was possible to evaluate the initial values for each of the aerodynamic derivatives. The relationships for determining the initial values for the stability and control derivatives from the linear state space representation are provided below ${ }^{69}$ :

$$
\begin{gathered}
C_{D \alpha}=\frac{m}{\bar{q}_{0} S}\left(g-A L G_{12}\right) \\
C_{D q}=-\frac{2 m V_{0}}{\bar{q}_{0} S \bar{c}} A L G_{13} \\
C_{D i_{H}}=-\frac{m}{\bar{q}_{0} S} B L G_{1} \\
C_{D 0}=\frac{T_{0} \cos \alpha_{0}}{\bar{q}_{0} S}-C_{D \alpha} \alpha_{0}-C_{D i_{H}} i_{H 0} \\
C_{L \alpha}=-\frac{m V_{0} A L G_{22}+T_{0}}{\bar{q}_{0} S} \\
C_{L q}=\left(1-A L G_{23}\right) \frac{2 m V_{0}^{2}}{\bar{q}_{0} S \bar{c}} \\
C_{L i_{H}}=-\frac{m V_{0}}{\bar{q}_{0} S} B L G_{2} \\
C_{L 0}=\frac{m g-T_{0} \sin \alpha_{0}}{\bar{q}_{0} S}-C_{L \alpha} \alpha_{0}-C_{L i_{H}} i_{H 0}
\end{gathered}
$$




$$
\begin{aligned}
& {\left[\begin{array}{c}
C_{m \alpha} \\
C_{m q} \\
C_{m i_{H}}
\end{array}\right]=\frac{1}{M_{4} \bar{q}_{0} S \bar{c}}\left[\begin{array}{c}
A L G_{32} \\
\frac{2 V_{0}}{\bar{c}} A L G_{33} \\
B L G_{3}
\end{array}\right]} \\
& C_{m 0}=-C_{m \alpha} \alpha_{0}-C_{m_{H}} i_{H} \\
& \left\lfloor\begin{array}{c}
C_{Y \beta} \\
C_{Y \delta_{A}} \\
C_{Y \delta_{R}}
\end{array}\right\rfloor=\frac{m V_{0}}{\bar{q}_{0} S}\left[\begin{array}{l}
A L T_{11} \\
B L T_{11} \\
B L T_{12}
\end{array}\right] \\
& {\left[\begin{array}{l}
C_{Y_{p}} \\
C_{Y_{r}}
\end{array}\right]=\frac{2 m V_{0}^{2}}{\bar{q}_{0} S b}\left[\begin{array}{l}
\left(A L T_{12}-\sin \alpha_{0}\right) \\
\left(A L T_{13}+\cos \alpha_{0}\right)
\end{array}\right]} \\
& {\left[\begin{array}{ll}
C_{l p} & C_{l r} \\
C_{n p} & C_{n r}
\end{array}\right]=\frac{2 V_{0}}{\bar{q}_{0} S b^{2}} M_{5}^{-1}\left[\begin{array}{ll}
A L T_{22} & A L T_{23} \\
A L T_{32} & A L T_{33}
\end{array}\right]} \\
& {\left[\begin{array}{c}
C_{l \beta} \\
C_{n \beta}
\end{array}\right]=\frac{1}{\bar{q}_{0} S b} M_{5}^{-1}\left[\begin{array}{l}
A L T_{21} \\
A L T_{31}
\end{array}\right]} \\
& \left\lfloor\begin{array}{ll}
C_{l \delta_{A}} & C_{l \delta_{R}} \\
C_{n \delta_{A}} & C_{n \delta_{R}}
\end{array}\right\rfloor=\frac{1}{\bar{q}_{0} S b} M_{5}^{-1}\left[\begin{array}{ll}
B L T_{21} & B L T_{22} \\
B L T_{31} & B L T_{32}
\end{array}\right]
\end{aligned}
$$

where the matrices $M_{4}$ and $M_{5}$ are defined as the following:

$$
\begin{gathered}
M_{4}=\frac{I_{x} I_{z}-J_{x z}^{2}}{\operatorname{det}(I)} \\
M_{5}=\frac{1}{\operatorname{det}(I)}\left[\begin{array}{cc}
I_{y} I_{z}-J_{y z}^{2} & J_{x y} J_{y z}+I_{y} J_{x z} \\
J_{x y} J_{y z}+I_{y} J_{x z} & I_{x} I_{y}-J_{x y}^{2}
\end{array}\right]
\end{gathered}
$$

$A L G$ and $B L G$ refer to the longitudinal linear model state and input matrices, respectively, and $A L T$ and $B L T$ refer to the lateral-directional linear model state and input matrices. The first and second subscripts indicate respectively the row and column number of a given element within the matrices.

It is important to note that the drag coefficients are difficult to accurately estimate from PID. The short period is the primary mode excited during a longitudinal maneuver that can be accurately estimated from the flight data, and the velocity is directly measured. Thus, the $\dot{V}$ 
equation is modeled separately based on the recorded velocity measurements. Additionally, the IMU measurements for $a_{x}$ are extremely noisy, which can have a significant effect on the estimation results. In this case, $c_{D_{q}}$ was considered negligible, and the other drag terms were estimated using the conversion the state space model as an initial point for optimization.

\subsubsection{CoDICE Stability and Control Derivative Modeling}

For the CoDICE modeling effort, the aerodynamic coefficients were redefined to account for the individual control surface effects. The aerodynamic coefficients for the CoDICE modeling are similar to those found in the nominal model as they are functions of the aircraft state vector ( $\left.\xi=[V, \alpha, \beta, p, q, r, \phi, \theta, \varphi, x, y, z]^{\mathrm{T}}\right)$ and input vector $\left(\delta=\left[\delta_{T}, \mathrm{i}_{\mathrm{H}}, \delta_{A}, \delta_{R}\right]^{\mathrm{T}}\right)$. The aerodynamic coefficients for the CoDICE aircraft model, however, have contributions from each of the six control surfaces for each coefficient. Specifically, within this effort, the aerodynamic coefficients were defined as the following:

$$
\begin{gathered}
C_{D}(\xi, \delta)=c_{D 0}+c_{D \alpha} \alpha+c_{D q} \frac{\bar{c}}{2 V} q+c_{D_{i_{H_{L}}}} i_{H_{L}}+c_{D_{i_{H_{R}}}} i_{H_{R}}+c_{D_{\delta A_{L}}} \delta_{A_{L}}+c_{D_{\delta A R}} \delta_{A_{R}}+c_{D_{\delta R_{L}}} \delta_{R_{L}}+c_{D_{\delta R_{R}}} \delta_{R_{R}} \\
C_{L}(\xi, \delta)=c_{L 0}+c_{L \alpha} \alpha+c_{L q} \frac{\bar{c}}{2 V} q+c_{L_{i_{H_{L}}}} i_{H_{L}}+c_{L_{i_{R}}} i_{H_{R}}+c_{L_{\delta A_{L}}} \delta_{A_{L}}+c_{L_{\delta A R}} \delta_{A_{R}}+c_{L_{\delta R_{L}}} \delta_{R_{L}}+c_{L_{\delta R_{R}}} \delta_{R_{R}} \\
C_{m}(\xi, \delta)=c_{m 0}+c_{m \alpha} \alpha+c_{m q} \frac{\bar{c}}{2 V} q+c_{m_{i H_{L}}} i_{H_{L}}+c_{m_{i H_{R}}} i_{H_{R}}+c_{m_{\delta A_{L}}} \delta_{A_{L}}+c_{m_{\delta A R}} \delta_{A_{R}}+c_{m_{\delta R_{L}}} \delta_{R_{L}}+c_{m_{\delta R_{R}}} \delta_{R_{R}} \\
C_{Y}(\xi, \delta)=c_{Y 0}+c_{Y \beta} \beta+c_{Y p} \frac{b}{2 V} p+c_{Y r} \frac{b}{2 V} r+c_{Y_{H_{H_{L}}}} i_{H_{L}}+c_{Y_{i_{H_{R}}}} i_{H_{R}}+c_{Y \delta_{A_{L}}} \delta_{A_{L}}+c_{Y \delta_{A_{R}}} \delta_{A_{R}}+c_{Y \delta_{R_{L}}} \delta_{R_{L}}+c_{Y \delta_{R_{R}}} \delta_{R_{R}} \\
C_{l}(\xi, \delta)=c_{l 0}+c_{l \beta} \beta+c_{l p} \frac{b}{2 V} p+c_{l r} \frac{b}{2 V} r+c_{l_{i_{H_{L}}}} i_{H_{L}}+c_{l_{i_{H_{R}}}} i_{H_{R}}+c_{l \delta_{A_{L}}} \delta_{A_{L}}+c_{l \delta_{A_{R}}} \delta_{A_{R}}+c_{l \delta_{R_{L}}} \delta_{R_{L}}+c_{l \delta_{R_{R}}} \delta_{R_{R}} \\
C_{n}(\xi, \delta)=c_{n 0}+c_{n \beta} \beta+c_{n p} \frac{b}{2 V} p+c_{n r} \frac{b}{2 V} r+c_{n i_{H_{L}}} i_{H_{L}}+c_{n i_{H_{R}}} i_{H_{R}}+c_{n \delta_{A_{L}}} \delta_{A_{L}}+c_{n \delta_{A_{R}}} \delta_{A_{R}}+c_{n \delta_{R_{L}}} \delta_{R_{L}}+c_{n \delta_{R_{R}}} \delta_{R_{R}}
\end{gathered}
$$

The nominal stability and control derivative optimization process was completed prior to the identification of the "unknown" control derivatives represented in Equations 4.52-4.57. It was assumed that these components have been optimized using the nominal flight data sets, leaving only the new "unknown" coefficients to be optimized. These "unknown" aircraft control derivatives had to be determined by converting the identified CoDICE linear state space model to provide a starting point for optimization. The conversion formulas for the "unknown" coefficients are represented by Equations $4.58-4.72$. 


$$
\begin{aligned}
& c_{D_{\delta A_{L}}}=\frac{-B_{\text {coup }}(1,3) m}{q_{0} S} \\
& c_{D_{\delta A_{R}}}=\frac{-B_{\text {coup }}(1,4) m}{q_{0} S} \\
& c_{D_{\delta R_{L}}}=\frac{-B_{\text {coup }}(1,5) m}{q_{0} S} \\
& c_{D_{\delta R_{R}}}=\frac{-B_{\text {coup }}(1,6) m}{q_{0} S} \\
& c_{L_{\delta A_{L}}}=\frac{-B_{\text {coup }}(2,3) m V_{0}}{q_{0} S} \\
& c_{L_{\delta A_{R}}}=\frac{-B_{\text {coup }}(2,4) m V_{0}}{q_{0} S} \\
& c_{L_{\delta R_{L}}}=\frac{-B_{\text {coup }}(2,5) m V_{0}}{q_{0} S} \\
& c_{L_{\delta R_{R}}}=\frac{-B_{\text {coup }}(2,6) m V_{0}}{q_{0} S} \\
& c_{m_{\delta A_{L}}}=\frac{B_{\text {coup }}(5,3)}{\left(I_{x x} I_{z z}-J_{x z}^{2}\right)|I| q_{0} S \bar{c}} \\
& c_{m_{\delta A_{R}}}=\frac{B_{\text {coup }}(5,4)}{\left(I_{x x} I_{z z}-J_{x z}^{2}\right)|I| q_{0} S \bar{c}} \\
& c_{m_{\delta R_{L}}}=\frac{B_{\text {coup }}(5,5)}{\left(I_{x x} I_{z z}-J_{x z}^{2}\right)|I| q_{0} S \bar{c}} \\
& c_{m_{\delta R_{R}}}=\frac{B_{\text {coup }}(5,6)}{\left(I_{x x} I_{z z}-J_{x z}^{2}\right)|I| q_{0} S \bar{c}}
\end{aligned}
$$

where $|I|$ is the determinant of the inertial matrix defined by Equation 4.28. 


$$
\begin{aligned}
c_{Y_{\delta E_{L}}} & =\frac{B_{\text {coup }}(3,1) m V_{0}}{q_{0} S} \\
c_{Y_{\delta E_{R}}} & =\frac{B_{\text {coup }}(3,2) m V_{0}}{q_{0} S} \\
{\left[\begin{array}{cc}
c_{l_{\delta E_{L}}} & c_{l_{\delta E_{R}}} \\
c_{n_{\delta E_{L}}} & c_{n_{\delta E_{R}}}
\end{array}\right] } & =\frac{1}{q_{0} S b} M_{5}^{-1}\left[\begin{array}{ll}
B_{\text {coup }}(4,1) & B_{\text {coup }}(4,2) \\
B_{\text {coup }}(6,1) & B_{\text {coup }}(6,2)
\end{array}\right]
\end{aligned}
$$

where $M_{5}^{-1}$ is defined by Equation 4.51. Similar to the scheme used to obtain the nominal stability and control derivatives, this optimization script aimed minimize the error between the output states of a simulated aircraft model and an actual set of flight data by adjusting the "unknown" control derivatives iteratively.

\subsection{WVU YF-22 Model Validation through Simulation Studies}

Once the nominal and CoDICE stability and control derivatives were identified, their accuracy was validated through simulation studies within a Simulink ${ }^{\circledR}$ environment. Specifically, a Simulink ${ }^{\circledR}$ scheme was used to evaluate how closely the simulated model performed when following a Virtual Leader scheme and when compared to actual flight data. For model validation, certain output states were considered a priority when being compared to measured output. Measured flight data used for the simulation study consisted of maneuvers not utilized for the identification process. This allowed for separate maneuvers to be used to validate the identified stability and control derivatives. Stabilator, aileron, and rudder doublets were passed through the identified stability and control derivatives and an error analysis was conducted between the measured responses and the simulated outputs. The angle of attack, pitch rate, roll rate, and yaw rate were among the states given the highest priority and evaluated for average error and standard deviation of the error. Several maneuvers for each control surface were used for the validation process (including those maneuvers under control surface "failure" conditions), and the error metrics were averaged for the differences between the dynamic modal results for measured and simulated output.

Once the model was validated, additional simulation studies were conducted where the OBES was employed to perform doublet maneuvers on selected control surfaces under both nominal and failure conditions as the aircraft was tracking the Virtual Leader path. Figure 20 shows the main simulation environment that was used for this effort. In Figure 20, the Virtual 
Leader GPS trajectory, GPS velocity, and pitch and bank angles were sent to the Controller subsystem, which contains the same controller as the on-board computer on the actual aircraft. The Controller subsystem block is shown in Figure 21.

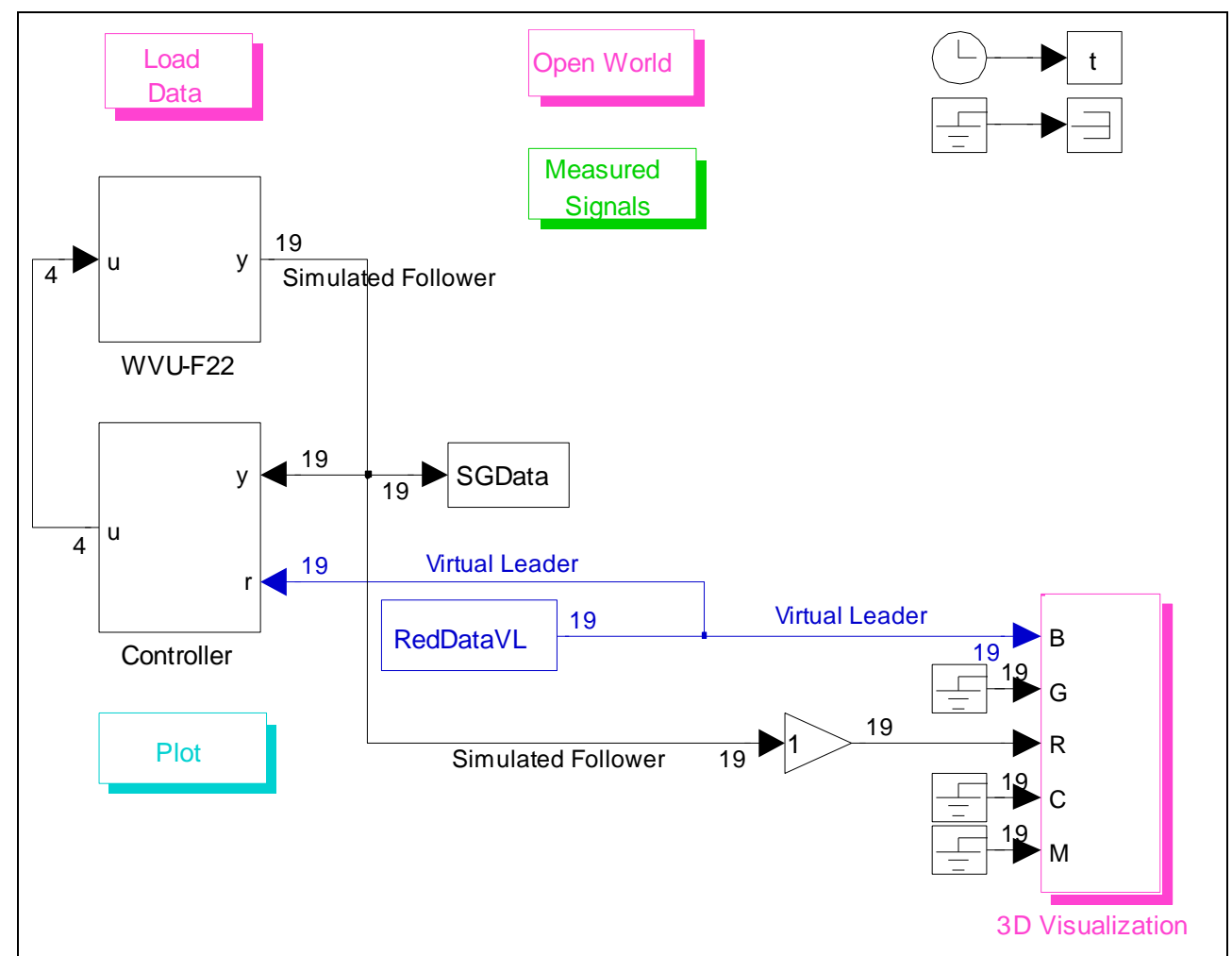

Figure 20: Simulink ${ }^{\circledR}$ Virtual Leader Simulation Environment

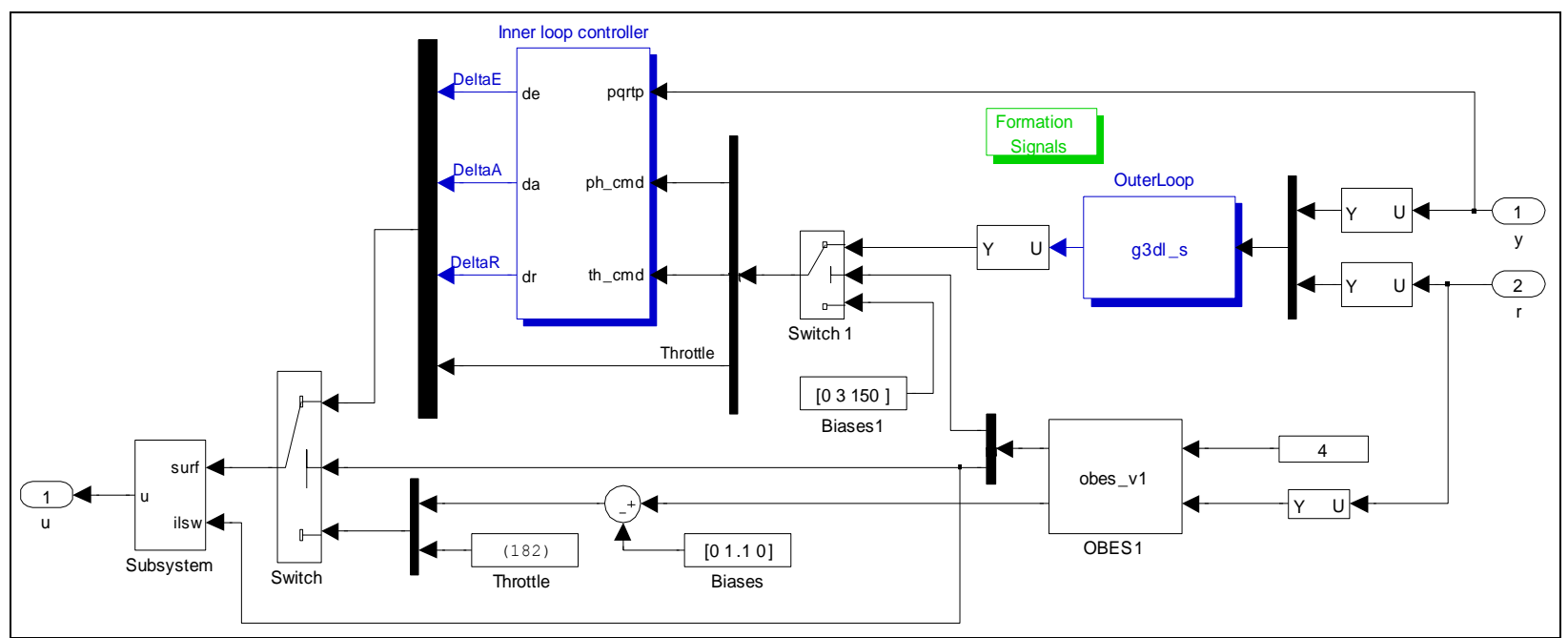

Figure 21: Controller Subsystem Block

The Controller subsystem in Figure 21 takes the VL information and sends it through the Outer Loop Controller, whose primary function is to direct the follower aircraft to follow the trajectory of the VL. The Outer Loop Controller sends the pitch angle, bank angle, and throttle information 
to the Inner Loop Controller, which is also accepting the follower aircraft behavior - pitch rate, roll rate, yaw rate, pitch angle, and bank angle - from the previous time step. From this information and the aircraft trim conditions, the Inner Loop Controller outputs deflection commands for the control surface pairs; these are then sent through actuator and engine models prior to running through the identified aircraft model. This process is conducted within the WVU YF-22 subsystem block shown in Figure 20. This subsystem outputs the next time step behaviors of the follower aircraft, which is then sent back into the Controller subsystem block to repeat the process described above. The follower information as well as the VL data is also sent to a 3-D Visualization block that allows the user to observe the general aircraft behavior visually.

Within the controller shown in Figure 21, there is another path that represents the OBES. To initiate the OBES for the VL path, a constant value of "4" or greater must be fed into the OBES subsystem. This will allow the follower aircraft to "bypass" the Outer Loop Controller essentially momentarily shutting off the tracking function of the follower aircraft - and allowing for the OBES to inject a specific maneuver during the flight path, similar to how actual flight tests will be conducted. Within this simulation path, failures may also be injected on control surfaces through the Subsystem block, prior to commands being sent through to the actuator models.

Studies were conducted through this simulation environment with the identified aircraft model to assess its performance. Specifically, a comparison of the GPS trajectory was observed between the VL track and the simulated follower track. The OBES was used to simulate injected maneuvers and provide a direct comparison to be evaluated against measured flight data. The average error and the standard deviation of the actual aircraft behavior from the simulated behavior were analyzed to see if the identified model accurately represented the actual aircraft.

\subsection{WVU YF-22 DATCOM Analysis}

The goal for this analysis was to utilize DATCOM-based ${ }^{41,42}$ methods to estimate some of the stability and control derivatives of the WVU YF-22, and compare values to the results obtained from PID. This analysis was used as a means of comparison for some primary stability and control derivatives of the WVU YF-22. DATCOM is rarely used as a validation tool for PID, but for the case of the WVU YF-22, wind tunnel analysis was not a feasible option. DATCOM also served as a means to predict the general range of the coefficient values, essentially by providing physical limitations or estimations for the optimization process of the stability and 
control derivatives for PID once it was deemed necessary. Additionally, the subsonic DATCOM charts did not have data for Mach number values below 0.2, so the information concerning this aircraft platform had to be extrapolated for a Mach of 0.12 . During initial stability and control derivative optimization efforts, stability and control derivatives representing the drag were finalizing at negative values, which is physically impossible. Additionally, a few coefficients were spiking to large magnitudes that also were questionable when applying engineering judgment.

DATCOM was developed specifically for application to large-scale, conventional, manned aircraft; therefore, to apply this methodology to the WVU YF-22 several assumptions concerning the body shape were made. The WVU YF-22 has a blended wing body, and as factors such as the static margin and $C_{L_{\alpha}}$ were calculated, it was essential that an accurate estimation of the wingbody behavior was used. The assumption of a double-delta cranked wing ${ }^{41}$ was used in the DATCOM-based calculations. Figure 22 illustrates the general geometry behind used for this assumption, and how it applies to the WVU YF-22. In this diagram, the double-delta cranked wing approximation is overlaid onto a CAD diagram of the WVU YF-22. The double-delta cranked wing was divided into two segments: inner and outer wings. From this, the chord lengths of the inner and outer wings were necessary for calculations of the static margin - typically one of the first steps in DATCOM-based analysis.

$$
\begin{gathered}
S=S_{i}+S_{o} \\
\lambda=\frac{c_{t}}{c_{r}}, \quad \lambda_{i}=\frac{c_{B}}{c_{r}}, \quad \lambda_{o}=\frac{c_{t}}{c_{B}} \\
A R=\frac{b^{2}}{S}, \quad A R_{i}=\frac{b_{i}^{2}}{S_{i}}, \quad A R_{o}=\frac{b_{o}^{2}}{S_{o}} \\
\bar{c}_{i}=\left(\frac{2}{3}\right)\left[\frac{1+\lambda_{i}+\lambda_{i}^{2}}{1+\lambda_{i}}\right], \bar{c}_{o}=\left(\frac{2}{3}\right)\left[\frac{1+\lambda_{o}+\lambda_{o}^{2}}{1+\lambda_{o}}\right] \\
\bar{c}=\frac{\bar{c}_{i} S_{i}+\bar{c}_{o} S_{o}}{S} \\
y_{M A C_{i}}=\frac{1}{3}\left(\frac{1+2 \lambda_{i}}{1+\lambda_{i}}\right) \frac{b_{i}}{2}, \quad y_{M A C_{o}}=\frac{1}{3}\left(\frac{1+2 \lambda_{o}}{1+\lambda_{o}}\right) \frac{b_{o}}{2} \\
y_{B}=\frac{b_{i}}{2}
\end{gathered}
$$




$$
\begin{gathered}
\bar{x}_{L E}=\frac{\left(y_{M A C_{i}} \tan \Lambda_{L E_{i}}\right) S_{i}+\left(y_{B} \tan \Lambda_{L E_{o}}+y_{M A C_{o}} \tan \Lambda_{L E_{o}}\right) S_{o}}{S} \\
y_{M A C}=\frac{y_{M A C_{i}} S_{i}+\left(y_{B}+y_{M A C_{o}}\right) S_{o}}{S}
\end{gathered}
$$

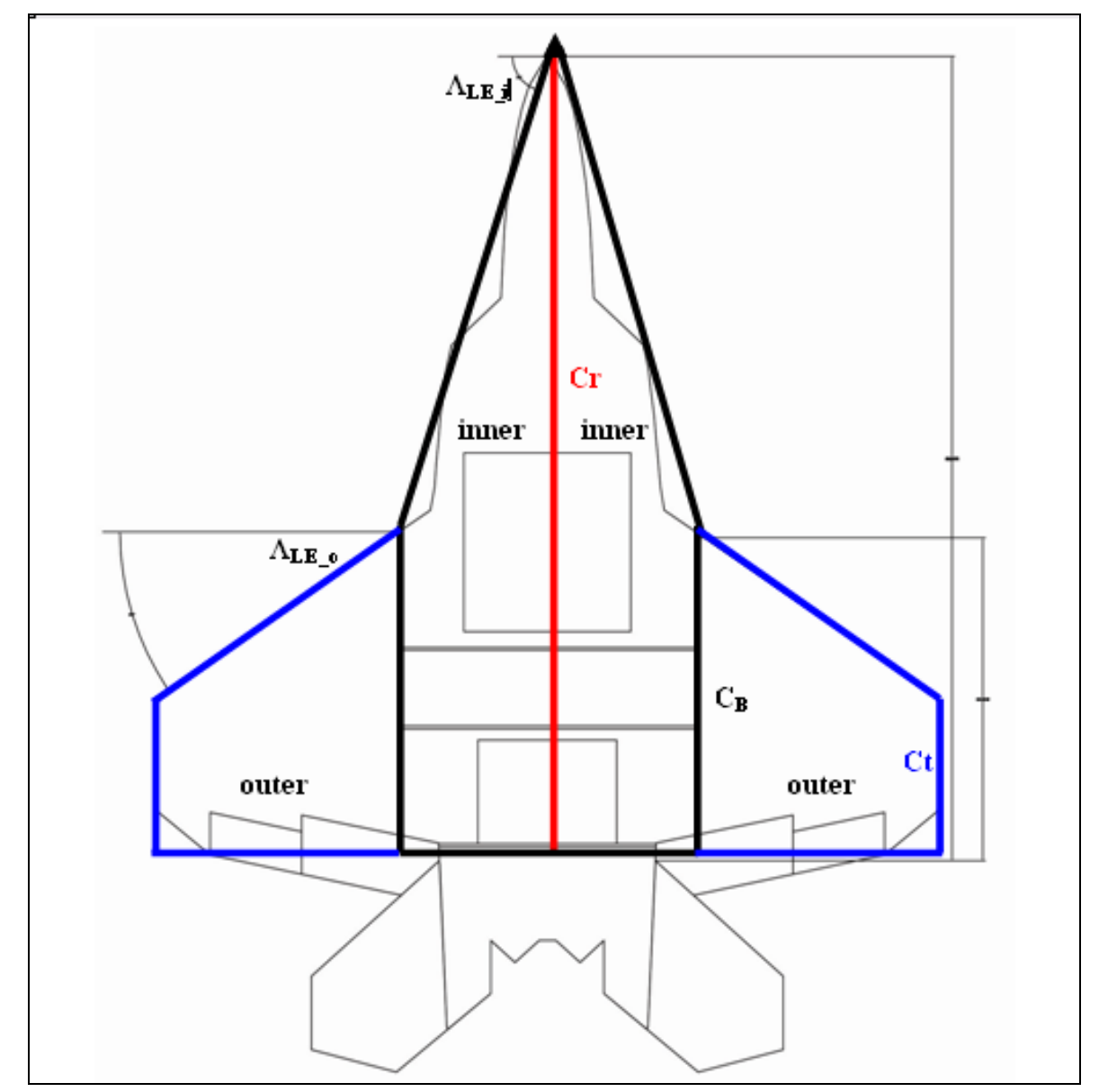

Figure 22: DATCOM Double-Delta Cranked Wing Approximation of WVU YF-22

The aerodynamic center and center of gravity were divided by the chord length of the aircraft to obtain $\bar{x}_{A C}$ and $\bar{x}_{C G}$, respectively. Using these values, the static margin of the aircraft was determined using Equation 4.82.

$$
S M=\bar{x}_{A C}-\bar{x}_{C G}
$$

Table 3 lists the primary dimensions of the YF-22 aircraft required for the static margin calculation as well as within the DATCOM formulas. These values were obtained from CAD drawings and verified with physical measurements of the actual aircraft. The subscripts ,O' and ,i’ refer to the "outer" and "inner" wing panels, respectively. 
Table 3: Geometric Parameters of the WVU YF-22

\begin{tabular}{|c|c|c|}
\hline \multicolumn{3}{|c|}{ DOUBLE DELTA - CRANKED WING } \\
\hline Dimension & Value & Units \\
\hline $\boldsymbol{C}_{\boldsymbol{r}}$ & 68.25 & (in) \\
\hline $\boldsymbol{C}_{\boldsymbol{B}}$ & 27.90 & (in) \\
\hline $\boldsymbol{C}_{\boldsymbol{t}}$ & 10.14 & (in) \\
\hline $\boldsymbol{\Lambda}_{\text {LEo }}$ & 0.59 & (rad) \\
\hline $\boldsymbol{\Lambda}_{\mathbf{L E i}}$ & 1.27 & (rad) \\
\hline $\boldsymbol{\lambda}$ & 0.15 & \\
\hline $\boldsymbol{\lambda}_{\boldsymbol{i}}$ & 0.41 & \\
\hline $\boldsymbol{\lambda}_{\boldsymbol{o}}$ & 0.36 & \\
\hline $\boldsymbol{b}$ & 67.19 & (in) \\
\hline $\boldsymbol{b}_{\boldsymbol{i}}$ & 25.44 & (in) \\
\hline $\boldsymbol{b}_{\boldsymbol{o}}$ & 41.75 & (in) \\
\hline $\boldsymbol{S}$ & 2017.10 & $\left(\right.$ in $\left.^{2}\right)$ \\
\hline $\boldsymbol{S}_{\boldsymbol{o}}$ & 794.16 & $\left(\right.$ in $\left.^{2}\right)$ \\
\hline $\boldsymbol{S}_{\boldsymbol{i}}$ & 1222.94 & $\left(\right.$ in $\left.^{2}\right)$ \\
\hline $\boldsymbol{A} \boldsymbol{R}_{\boldsymbol{o}}$ & 2.20 & \\
\hline $\boldsymbol{A} \boldsymbol{R}_{\boldsymbol{i}}$ & 0.53 & \\
\hline $\mathbf{R e}$ & $1.48 \times 10^{6}$ & \\
\hline
\end{tabular}

The center of gravity on the WVU YF-22 aircraft was determined to be located 49.0 inches back from the tip of the nose. Once the static margin was calculated for the WVU YF-22, DATCOM was used to estimate the downwash parameter on the horizontal tail.

Table 4 lists the primary dimensions of the WVU YF-22 horizontal tail, specifically the entire stabilator control surface. These parameters were utilized in the determination of several of the stability and control derivatives for the WVU YF-22.

Table 4: Geometric Properties of the WVU YF-22 Horizontal Tail

\begin{tabular}{|c|c|c|}
\hline \multicolumn{3}{|c|}{ HORIZONTAL TAIL } \\
\hline$\Lambda_{\mathrm{LE}}$ & 42.00 & $(\mathrm{deg})$ \\
\hline$\Lambda_{0.5}$ & 27.00 & $(\mathrm{deg})$ \\
\hline$\Lambda_{\mathrm{H}}$ & 0.50 & \\
\hline $\mathrm{S}_{\mathrm{H}}$ & 270.42 & $\left(\mathrm{in}^{2}\right)$ \\
\hline $\mathrm{AR}_{\mathrm{H}}$ & 2.50 & \\
\hline
\end{tabular}

Table 5 lists the vertical tail characteristics, including the rudders. The contributions of the vertical tail were implemented in the determination of several of the stability and control derivatives for the WVU YF-22. 
Table 5: Geometric Properties of the WVU YF-22 Vertical Tail

\begin{tabular}{|c|c|c|}
\hline \multicolumn{3}{|c|}{ VERTICAL TAIL } \\
\hline $\mathrm{Cr}$ & 18.13 & (in) \\
\hline $\mathrm{Ct}$ & 5.50 & (in) \\
\hline$\lambda$ & 0.30 & \\
\hline$\Lambda_{\mathrm{LE}}$ & 24.00 & (deg) \\
\hline$\Lambda_{0.25}$ & 13.00 & (deg) \\
\hline$\Lambda_{0.5}$ & 1.00 & (deg) \\
\hline $\mathrm{AR}$ & 1.73 & \\
\hline
\end{tabular}

For the complete set of equations used for the calculation of the selected stability and control derivatives, please refer to Appendix A.

\subsection{Confidence Measures of WVU YF-22 Model}

Parameter estimation from flight data is a common practice to obtain a linear representation for an aircraft. These values are only estimates so it becomes necessary to assess their reliability, specifically when they are to be used with an adaptive flight control system ${ }^{88}$. In addition to basic engineering judgment, which may be based on a priori knowledge of the system, a common method used for analysis of the estimate reliability is a scatter analysis paired with Cramer-Rao Bounds. The Cramer-Rao bound provides the lower bound on the error variance of the best estimator for a deterministic parameter ${ }^{89}$. The following paragraphs describe the basis for a scatter analysis and the Cramer-Rao bound determination.

When data is collected for several maneuvers at a given flight condition, the scatter of the estimates provides an indication of accuracy. Maneuvers may also be evaluated over a gradually changing flight condition, i.e. increasing angle of attack. An advantage of a scatter analysis is that it measures the actual performance that statistical measures try to predict ${ }^{88}$. For the best results in a scatter analysis, a larger number of data points should be available. If there are a small number of maneuvers used for comparison, it is more difficult to gauge the accuracy of the estimates. Another disadvantage of scatter analysis is that it does not account for consistent biases in the measurements. These biases could result from a poor moment of inertia estimate or a sensor bias.

An example discussed in Reference 88 provides a sample analysis of rudder and aileron maneuvers used to estimate a stability derivative. When the data is segregated into rudder and aileron maneuvers, it is clear that the aileron maneuvers provided the "best" scatter of the estimations. Figure 23 shows a scatter plot with segregated rudder and aileron maneuvers, and 
highlights that the ailerons provided the most consistent estimates. The scatter analysis provides the lower bound on the error of the estimates: estimates could be worse than predicted with the scatter plot but they will rarely be better.

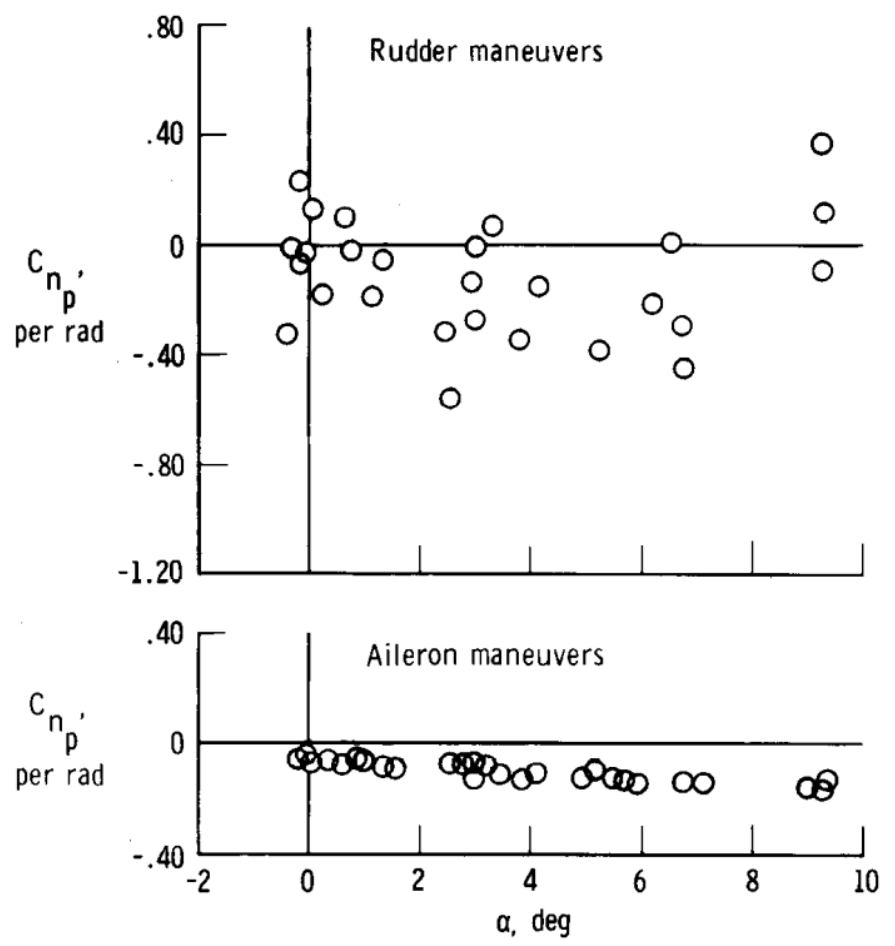

Figure 23: Estimates Segregated by Input Type [Reproduced from Reference 88]

The Cramer-Rao bound is based on the Cramer-Rao inequality as defined by Equation 4.83.

$$
E\left\{\left(\hat{\xi}-\xi_{t}\right)\left(\hat{\xi}-\xi_{t}\right)^{*} \mid \xi_{t}\right\} \geq M\left(\xi_{t}\right)^{-1}
$$

where $M\left(\xi_{t}\right)$ is the Fisher information matrix, $E\left\{\left(\hat{\xi}-\xi_{t}\right)\left(\hat{\xi}-\xi_{t}\right)^{*} \mid \xi_{t}\right\}$ is the covariance of the estimate, and $\hat{\xi}$ is a function of the system response only. For unbiased estimators, $b\left(\xi_{t}\right)$ is zero so the variance of $\hat{\xi}$ is equivalent to the left-hand side of Equation 4.83. The Cramer-Rao bound is also based on the uncertainty ellipsoid, which is an approximation of the confidence region based on a "statistical or geometric picture of the relationship between the Cramer-Rao bounds, sensitivities, and correlations" ${ }^{\prime 88}$. The Cramer-Rao bound is greatly affected by the colored measurement noise and modeling error in flight data, and corrections for these must be implemented to avoid highly erroneous bound estimates. If the colored noise and modeling error 
is not accounted for, a "fudge factor" between 5 and 10 may be included. This is ad hoc in nature, but this method and factor value has been justified over a large series of cases ${ }^{88}$.

For this study, the confidence intervals of the estimated linear state space parameters were evaluated at the $95 \%$ level. The $95 \%$ confidence interval for each estimated parameter is an interval computed from sample data that has a $95 \%$ probability of producing an interval containing the "true" parameter value ${ }^{90}$. The Cramer-Rao bounds were implemented to determine the confidence intervals of the estimates based on the lower bound. The Cramer-Rao bounds were calculated for least squares regression with the following regression model ${ }^{51}$ :

$$
y=x p
$$

where $x$ is a matrix of column regressors, $p$ is a vector of parameter estimates, and $y$ is the model output vector. The model output vector is compared to the measured states output vector $z$ which is utilized in the calculation of the Cramer-Rao bounds.

$$
\begin{gathered}
v=z-y \\
h=\frac{v^{\prime} v}{n-m}
\end{gathered}
$$

where $n$ and $m$ are the number of rows and columns of the column regressor matrix $x$.

$$
G=x^{\prime} x
$$

The inverse of the input matrix $G$ is calculated using singular value decomposition to obtain matrix $G_{i}$. Equation 4.88 was then used to calculate the Cramer-Rao bound.

$$
C r b=h G_{i}
$$

For a more detailed summary of this method and how it is applied to aircraft parameter estimation please refer to Reference 88 and 51.

\subsection{Propulsion Assisted Control Testbed (PACT) Research Platform}

The WVU PACT research aircraft will be the basic future platform for the development of flight control laws at WVU, specifically for propulsion assisted control applications. Before these control laws may be developed and applied to the autonomous flight, an accurate mathematical model of the aircraft must be identified and tested via simulation studies. The 
following sections describe the WVU PACT aircraft platform and its capabilities for flight testing.

\subsubsection{PACT Aircraft System}

The PACT is an unmanned radio-controlled aircraft designed, constructed, and instrumented at West Virginia University. The fuselage is a carbon fiber and fiberglass composite body, and the aircraft has a T-tail and two ducted fan motors. The motors will be mounted at different locations on several variations of the aircraft including on the aft fuselage, under the wings, and on the vertical tail. For this study, the variation of the PACT aircraft with the engines mounted on the aft fuselage was used. The propulsion system is based on Lander Technologies $9 \mathrm{~mm}$ ducted fans with a Medusa brushless in-runner motor. Each motor produces approximately $5 \mathrm{lbs}$ of static thrust (10 lbs total), providing the aircraft with an estimated total of $6 \mathrm{lbs}$ of dynamic thrust in flight. The version of the PACT aircraft used in this study includes winglets and does not have flaps for increased lift on takeoff and landing. The primary control surfaces - ailerons, elevators, and a rudder - are all commanded using digital servos. Figure 24 shows the WVU PACT aircraft.

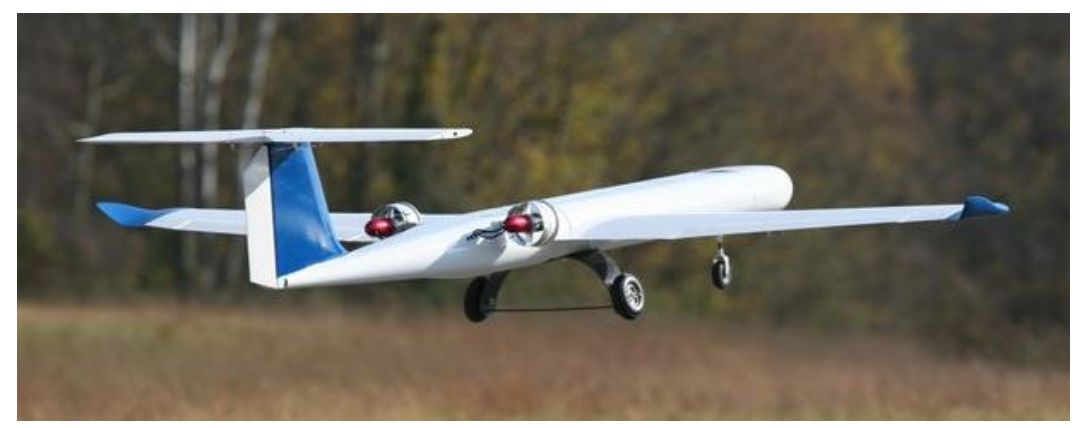

Figure 24: WVU PACT Aircraft

\subsubsection{PACT Geometric Parameters}

The PACT wings were divided into inner and outer wing section components. The geometric properties of the inner and outer wing sections of the PACT are listed in Table 6. The inner wing section also includes the portion of the wing that lies within the fuselage. There is no leading edge sweep on the wings of the PACT; however, there is a swept-forward trailing edge on the outer wings. Because of this, the sweep angle of the half-chord and quarter-chord are represented as negative values on the outer wing. For the outer wing analysis, the left and right outer sections are "combined" to form the total outer wing. 
Table 6: Inner and Outer Wing Geometric Parameters

\begin{tabular}{|c|c|c|}
\hline Dimension & Value & Units \\
\hline \multicolumn{3}{|l|}{ Inner Wing } \\
\hline$A R$ & 2.29 & - \\
\hline$\Lambda_{L E}$ & 0.00 & (deg) \\
\hline$\Lambda_{0.25}$ & 0.00 & (deg) \\
\hline$\Lambda_{0.5}$ & 0.00 & (deg) \\
\hline $\mathrm{Cr}$ & 14.00 & (in) \\
\hline$C t$ & 14.00 & (in) \\
\hline$\lambda$ & 1.00 & - \\
\hline $\bar{c}$ & 14.00 & (in) \\
\hline$b$ & 32.00 & (in) \\
\hline$s$ & 448.00 & $\left(\mathrm{in}^{2}\right)$ \\
\hline$y_{M A C}$ & 8.00 & (in) \\
\hline Dimension & Value & Units \\
\hline \multicolumn{3}{|l|}{ Outer Wing } \\
\hline$A R$ & 4.04 & - \\
\hline$\Lambda_{L E}$ & 0.00 & (deg) \\
\hline$\Lambda_{0.25}$ & -2.98 & (deg) \\
\hline$\Lambda_{0.5}$ & -5.80 & (deg) \\
\hline $\mathrm{Cr}$ & 14.00 & (in) \\
\hline$C t$ & 9.75 & (in) \\
\hline$\lambda$ & 0.70 & - \\
\hline $\bar{c}$ & 12.00 & (in) \\
\hline$b$ & 48.00 & (in) \\
\hline$S$ & 570.00 & $\left(\mathrm{in}^{2}\right)$ \\
\hline$y_{M A C}$ & 11.28 & (in) \\
\hline
\end{tabular}

Figure 25 shows the front view of the PACT with the total wing, horizontal tail, and vertical tail spans represented.

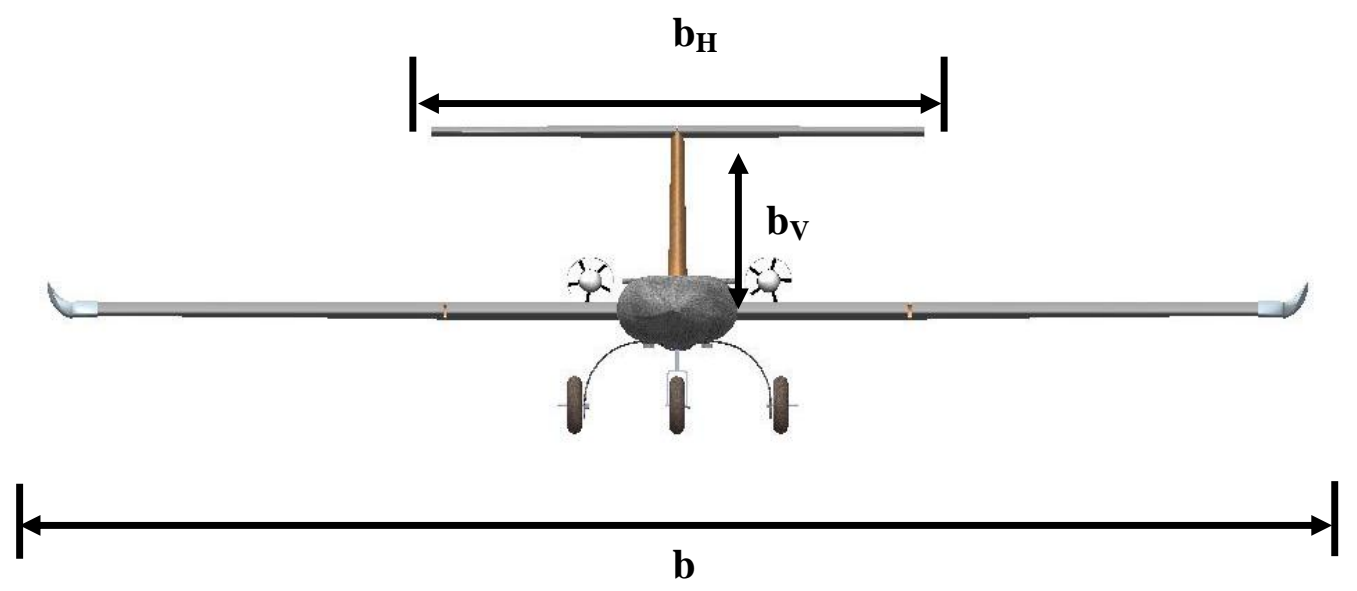

Figure 25: PACT Total Wing, Horizontal Tail, and Vertical Tail Spans 
Table 7 shows the geometric properties of the total (combined) wing span of the PACT, and Table 8 shows the horizontal and vertical tail geometric parameters.

Table 7: PACT Total Wing Geometric Parameters

\begin{tabular}{|c|c|c|}
\hline Dimension & Value & Units \\
\hline Total Wing Estimation & & - \\
\hline $\boldsymbol{A R}$ & 6.76 & (deg) \\
\hline $\boldsymbol{\Lambda}_{\boldsymbol{L E}}$ & 0.00 & (deg) \\
\hline $\boldsymbol{\Lambda}_{\boldsymbol{0 . 2 5}}$ & -1.77 & (deg) \\
\hline $\boldsymbol{\Lambda}_{\boldsymbol{O} .5}$ & -3.58 & (in) \\
\hline $\boldsymbol{C r}$ & 14.00 & (in) \\
\hline $\boldsymbol{C t}$ & 9.75 & - \\
\hline $\boldsymbol{\lambda}$ & 0.70 & (in) \\
\hline $\bar{c}$ & 12.00 & (in) \\
\hline $\boldsymbol{b}$ & 80.25 & (in ${ }^{2}$ \\
\hline $\boldsymbol{S}$ & 952.97 & (in) \\
\hline $\boldsymbol{y}_{\text {MAC }}$ & 18.87 & - \\
\hline $\boldsymbol{M a c h}$ & 0.073 & \\
\hline
\end{tabular}

Table 8: PACT Horizontal and Vertical Tail Geometric Parameters

\begin{tabular}{|c|c|c|}
\hline \multicolumn{3}{|c|}{ HORIZONTAL TAIL (including elevators) } \\
\hline$\Lambda_{L E}$ & 5.04 & (deg) \\
\hline$\Lambda_{0.5}$ & 0.84 & (deg) \\
\hline$\Lambda_{0.25}$ & 2.95 & (deg) \\
\hline$C_{r}$ & 9.63 & (in) \\
\hline$C_{t}$ & 7.13 & (in) \\
\hline$\lambda$ & 0.74 & - \\
\hline$b_{H}$ & 34.00 & (in) \\
\hline$S_{H}$ & 288.94 & $\left(\right.$ in $\left.^{2}\right)$ \\
\hline $\boldsymbol{A} \boldsymbol{R}_{H}$ & 4.00 & - \\
\hline \multicolumn{3}{|c|}{ VERTICAL TAIL (including rudder) } \\
\hline$\Lambda_{L E}$ & 21.09 & (deg) \\
\hline$\Lambda_{0.5}$ & 10.30 & (deg) \\
\hline$\Lambda_{0.25}$ & 15.24 & (deg) \\
\hline$C_{r}$ & 13.36 & (in) \\
\hline$C_{t}$ & 9.51 & (in) \\
\hline$\lambda$ & 0.71 & - \\
\hline $\boldsymbol{b}_{V}$ & 11.00 & (in) \\
\hline$S_{V}$ & 251.56 & $\left(\mathrm{in}^{2}\right)$ \\
\hline $\boldsymbol{A R}_{V}$ & 1.92 & - \\
\hline
\end{tabular}




\subsubsection{PACT Airfoils}

Two NACA airfoils were used for the PACT wings and tail: NACA 2410 for the aircraft wing and NACA 0009 for the horizontal and vertical tails. The aerodynamic characteristics ${ }^{91}$ of these wing sections were utilized in a DATCOM analysis of this aircraft (described in Appendix A). Figures 26 and 27 show the airfoil profiles as well as the location of the maximum thickness at $25 \%$ of the chord length for both airfoils.

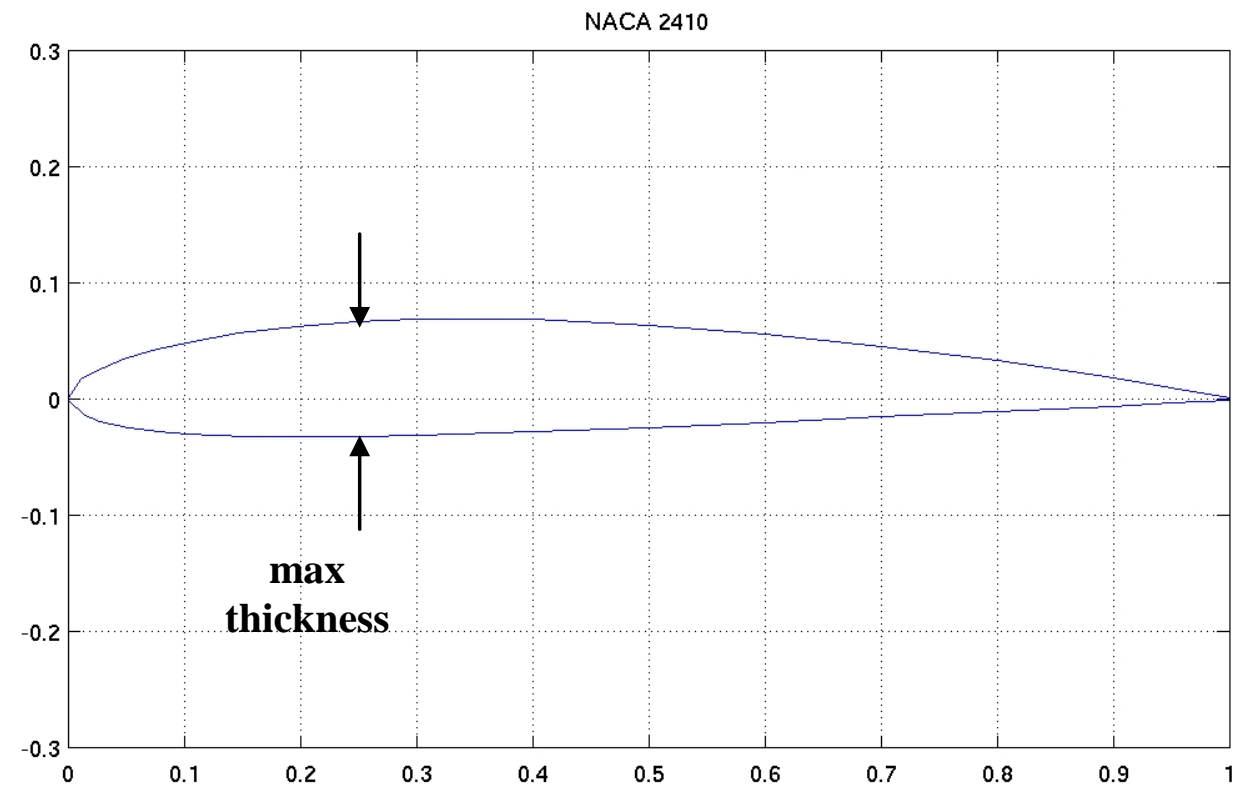

Figure 26: PACT Main Wing Airfoil

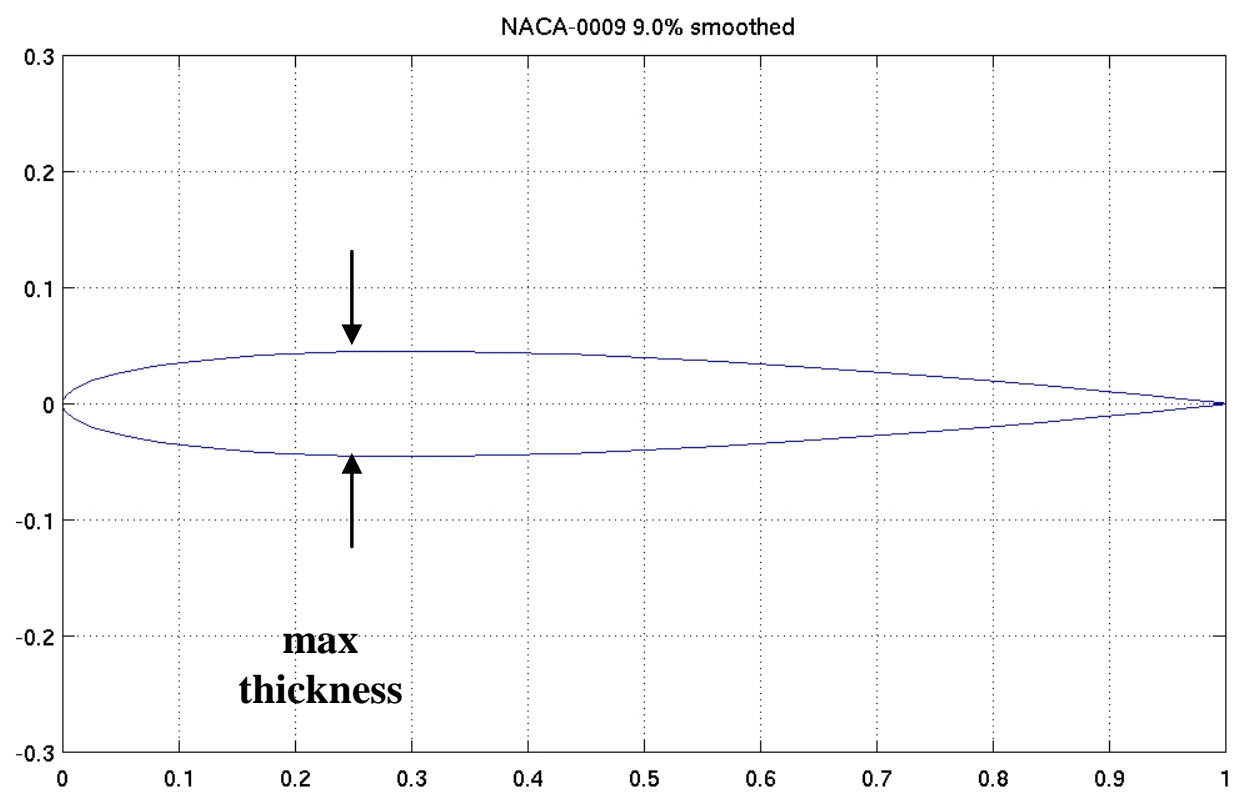

Figure 27: PACT Horizontal and Vertical Tail Airfoil 


\subsubsection{PACT On-Board Computer}

A new computer was developed for the PACT aircraft specifically to test fault-tolerant flight control schemes. The Advanced Research Integrated Avionic (ARIA) System was developed by researchers at WVU and is comprised of a stack of three Printed Circuit Boards (PCBs), each providing a specific function to the system. The top board is a Single Board Computer (SBC) with an integrated data acquisition, the middle board is a PC-104 power supply with additional RS-232 ports, and the bottom board is a custom board designed to act as a sensor interface and signal distribution system. The ARIA system is integrated with a MEMS IMU on the bottom PCB. When compared to the flight computer system for the WVU YF-22, there was a $50 \%$ reduction in volume and the ARIA saves about $5 \mathrm{lbs}$ of weight due to the ability to remove the vertical gyro and replace its function of measuring flight path angles with a GPS/INS sensor fusion algorithm ${ }^{92}$. Figure 28 shows the ARIA System developed for the PACT aircraft.

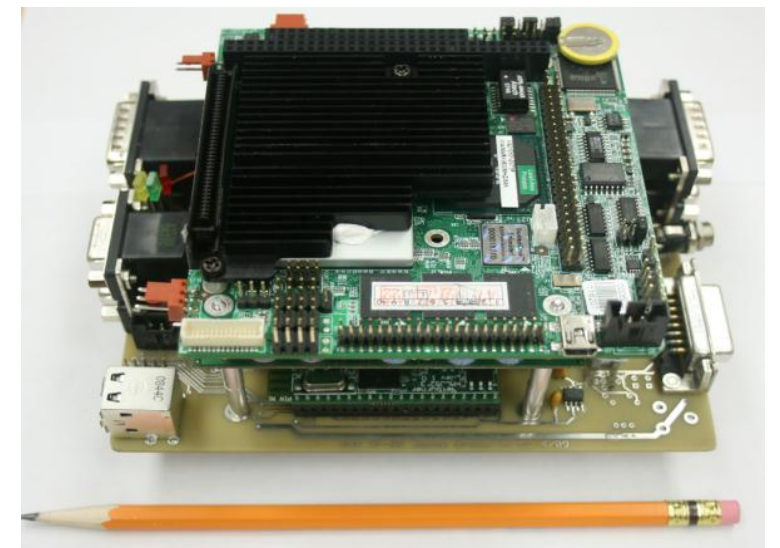

Figure 28: ARIA System [Reproduced from Reference 92]

According to Reference 92, the ARIA system has the following design features:

A 32-bit $66 \mathrm{MHz}$ Freescale ColdFire MOD $5213^{\circledR}$ microprocessor with a realtime operating system is integrated into the custom PCB and used to tackle much of the communications workload within the system. The MOD 5213 allows task prioritization with seven interrupt levels. Its tasks include interfacing with the MEMS IMU, reading control command signals from the ground pilot generated by the $R / C$ receiver, and writing control commands as prescribed by the on-board flight control software. The microprocessor receives inertial information through a serial peripheral interface (SPI) from Analog Device's ADIS-16355 High Precision Tri-Axis IMU. The inertial information recorded includes three-axis acceleration, and angular rates. The entire 
function of reading and writing control command signals is described in detail in the next section of this paper.

The Diamond System's $800 \mathrm{MHz}$ Athena II general purpose SBC with sixteen 16bit integrated $A / D$ serves as the main flight computer within the ARIA system. Along with a PC/104 compatible power supply, a total of 6 serial ports are available for communicating with various devices. Two serial ports are devoted for communication with the embedded microprocessor, while an additional two are utilized to interface with a GPS unit and a RF modem leaving two available for auxiliary external devices. One proposed expansion would be the use of multiple GPS receivers. Using the generalpurpose SBC effectively enhances the on-board computational resources and provides additional interfaces without the need for customized hardware design.

\subsubsection{PACT Sensors and Communication Hardware}

The WVU PACT aircraft is instrumented with a complete suite of sensors for measuring a variety of flight data parameters. A MEMS IMU integrated on the PCB provides measurements of the linear accelerations and angular rates. A GPS receiver provides position and velocity information in three dimensions with respect to the earth reference frame, and a GPS/INS sensor fusion algorithm provides pitch and roll information for the aircraft. Some variations of the PACT aircraft may also contain a vertical gyroscope to record the pitch and roll information. Vanes designed and manufactured in-house were attached to potentiometers to provide measurements of the angle of attack and sideslip angle. The control surface deflections were determined through the measurement of the pilot inputs. A relationship between the commanded input and the actuator model was established to determine the control surface deflections. The following sensors were used onboard the PACT aircraft for PID:

- Inertial Measurement Unit (ADIS16355 ${ }^{\circledR}$ IMU), providing 12-bit measurements for the accelerations $a_{x}, a_{y}, a_{z}$ (range $\pm 10 \mathrm{~g}$ ), and the angular rates $p, q$, and $r$ (range $\pm 150^{\circ} / \mathrm{sec}$ ).

- Vertical gyro (Goodrich-VG34), providing measurements for the pitch and roll Euler's angles $(\theta$ and $\phi)$ with ranges of $\pm 60^{\circ}$ and $\pm 90^{\circ}$, respectively.

- GPS receiver (Novatel-OEM1), providing measurements for $x, y, z, V_{x}, V_{y}, V_{z}$ with respect to an earth reference frame.

- Air Data Probe, providing measurements of flow angles $\alpha$ and $\beta$, with ranges of $\pm 30^{\circ}$. 
- Absolute and Dynamic pressure sensors (Honeywell ASCX15AN and Honeywell ASCX01DN), with ranges of [0-15] and [0-1] PSI, respectively. Both sensors are connected to the nose probe providing measurements for $H$ and $V$.

- Temperature sensor (digital sensor under a $3.3 \mathrm{~V}$ supply).

\subsection{PACT PID Analysis from Flight Data}

A parameter identification study was conducted on the WVU PACT using a similar procedure to that used for the WVU YF-22 aircraft. Please refer to Sections 4.2.1, 4.3.2, and 4.4.1 describing the experiment design and nominal state space model and stability and control derivative identification for the WVU YF-22 for an overview of this process. For the PACT aircraft, elevators were the longitudinal control surface inputs replacing the stabilators in the YF22 model. Selected values obtained through PID were compared to results obtained through DATCOM, and DATCOM was again utilized to provide upper and lower bound estimates for stability and control derivative optimization. The PID modeling results were validated through a series of simulation studies with the estimated PACT mathematical model. A confidence interval analysis was also conducted on the state space parameter estimates for the PACT aircraft and is detailed in the results. 


\section{Results}

This section is divided into two major segments: WVU YF-22 and PACT modeling and simulation results. The WVU YF-22 modeling procedures were designed with the end goal of application within a fault-tolerant flight control system handling actuator failures on the primary control surfaces, while the PACT modeling procedure was designed with an end goal of obtaining a model representing the aircraft under nominal conditions with the thrust vector aligned with the longitudinal body axis. The methods found to be successful through research with the WVU YF-22 flight data were applied to obtain the nominal model of the PACT aircraft.

\subsection{WVU YF-22 Results}

\subsubsection{WVU YF-22 Linear State Space Model Identification Results}

The linear state space identification results are reported in two sections: nominal modeling and CoDICE modeling results. The nominal modeling results section details the specific flight maneuvers conducted, results from the Matlab ${ }^{\circledR}$ System Identification Toolbox analysis, and results from the output-error optimization of the linear state space model. Two different trials were conducted to obtain a nominal linear model for baseline control law design: utilize the output from the System Identification Toolbox, and utilize the model identified through the System Identification Toolbox and optimize it using the output-error optimization method. The CoDICE modeling results section details the specific flight maneuvers conducted and results from the Fourier Transform Regression approach to identify the state space cross-coupled terms.

\subsubsection{Nominal Modeling Results}

Parameter identification flight tests were conducted during 2008, 2009, and 2010 with the WVU YF-22 to estimate a nominal mathematical model of the aircraft. Specific maneuvers were performed with the control surfaces to excite the short period, Dutch roll, and roll responses of the aircraft. Doublet and 1-1-2-3 doublet maneuvers were performed on the stabilators to excite the short period, and rudder/aileron doublet combinations and aileron doublets were performed to excite the Dutch roll and roll modes. These maneuvers were performed both manually by a pilot and autonomously by on on-board excitation system. Figures 29 and 30 show samples of flight data used for parameter identification with a stabilator doublet and rudder/aileron doublet combination, respectively. These maneuvers were performed by the pilot and represent the type of data segmenting that was used in the identification process of the nominal state space model. 
Each maneuver performed during flight tests were segmented to show the input maneuver as well as the system dynamic response.

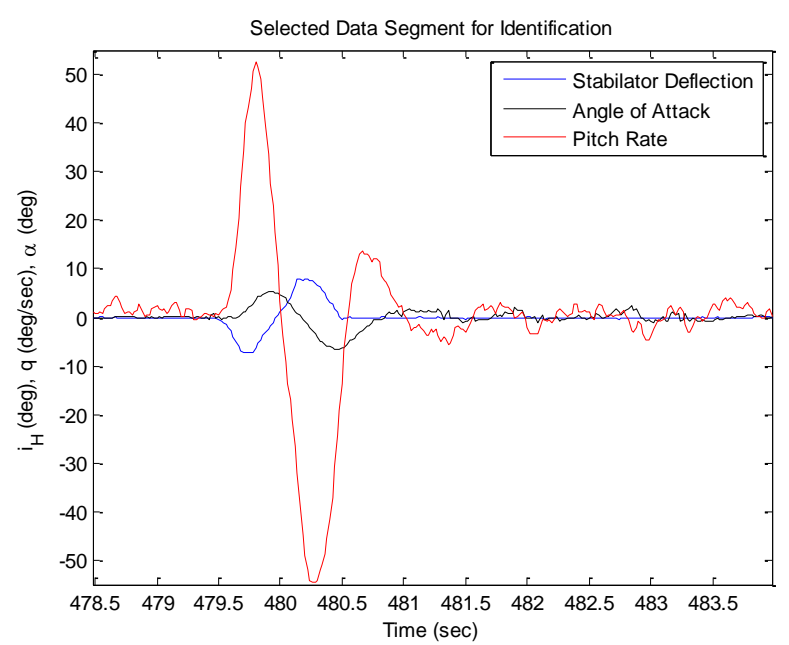

Figure 29: Flight Data Segment used for Longitudinal Linear Model Identification

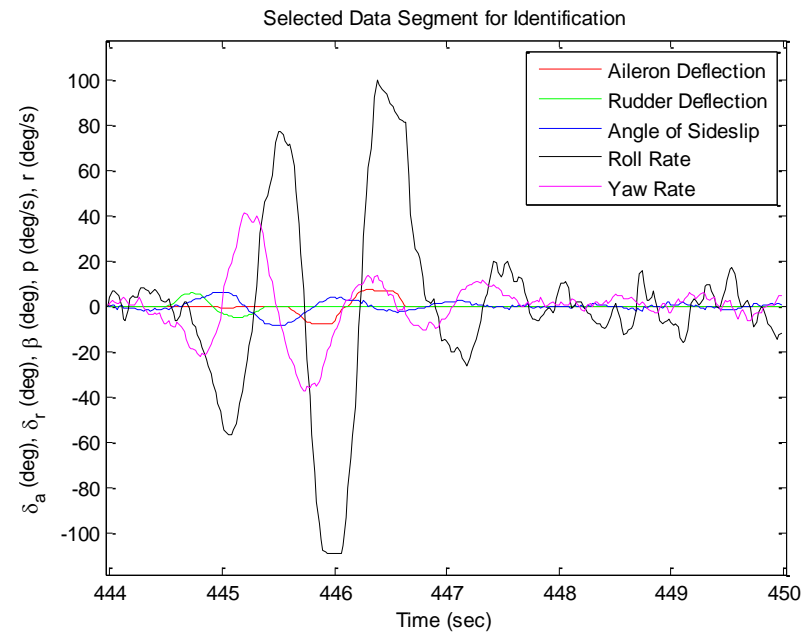

Figure 30: Flight Data Segment used for LateralDirectional Model Identification

For the autonomous flight tests, the Virtual Leader path was followed with the OBES injecting the maneuvers during the straight portions of the flight. Figure 31 shows the Virtual Leader track as it was designed over Jackson's Mill. The runway is located in the center of the flight path, with the straight legs parallel to the runway. During the autonomous flight tests, the aircraft tracked this trajectory, remaining within safe visual distance of the flight field.

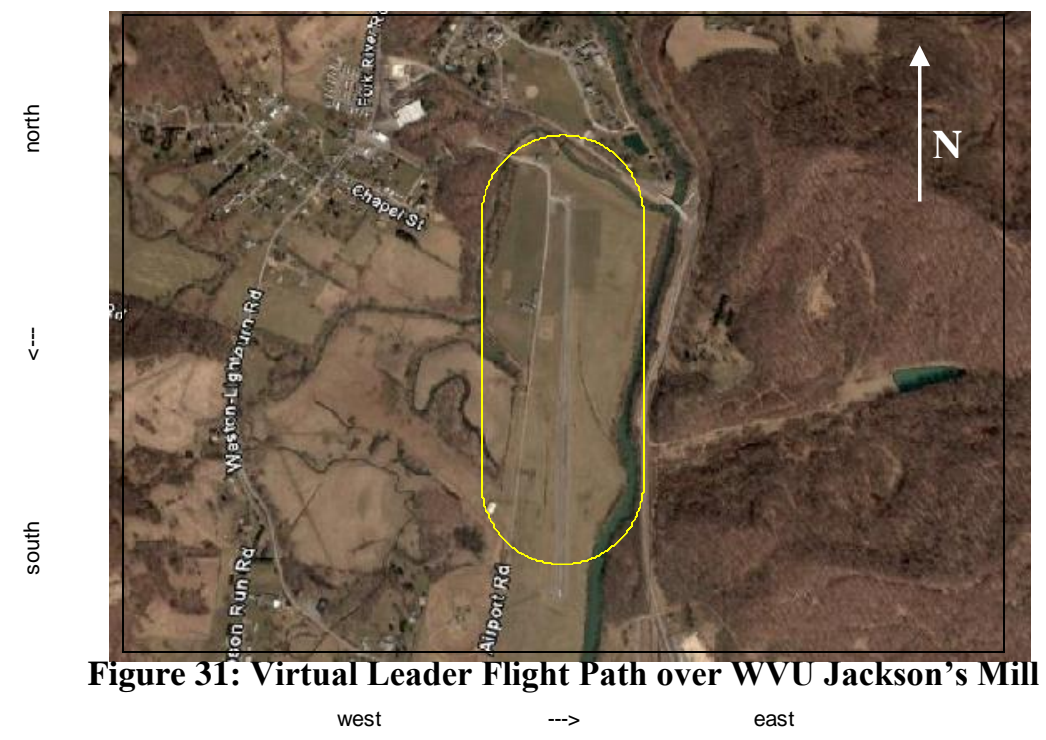

During the autonomous flight tests, the OBES performed the maneuvers during the straight portions of the laps at predefined locations. One second prior to the injection of the 
doublet maneuver, the outer loop controller was turned off. This disabled the GPS trajectory tracking and allowed for the actual aircraft dynamic to be observed following the maneuver injection. At 0.2 seconds prior to the injection of the maneuver, all of the control surfaces were set to trim to verify that they would not cause any additional excitation. Since the aircraft was already tracking a straight and level trajectory at the point of this implementation, typically the surfaces were already very close to their trim condition. This simply provided an additional means to ensure that they were at this point prior to the maneuver. Once the doublet maneuver was injected by the OBES, five to seven seconds were provided to allow the observation of the aircraft dynamic response before the outer loop controller (GPS tracking) was turned back on. Figure 32 shows a stabilator doublet performed by the OBES during an autonomous flight. Figure 33 shows the general layout of the OBES "mask" inside the controller that allows for the design of the maneuver to be performed during the flight.

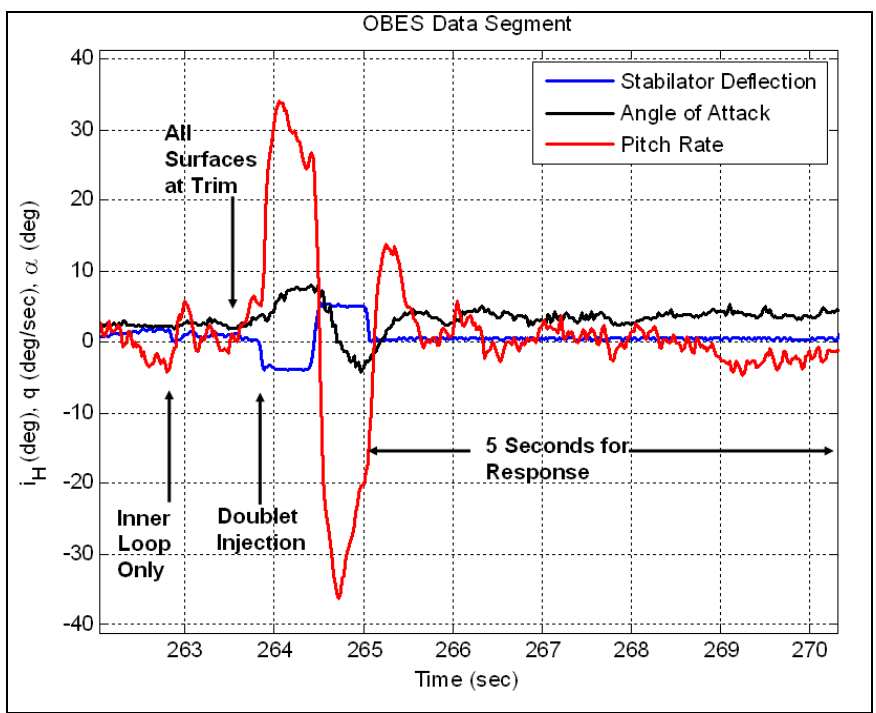

Figure 32: OBES Stabilator Doublet

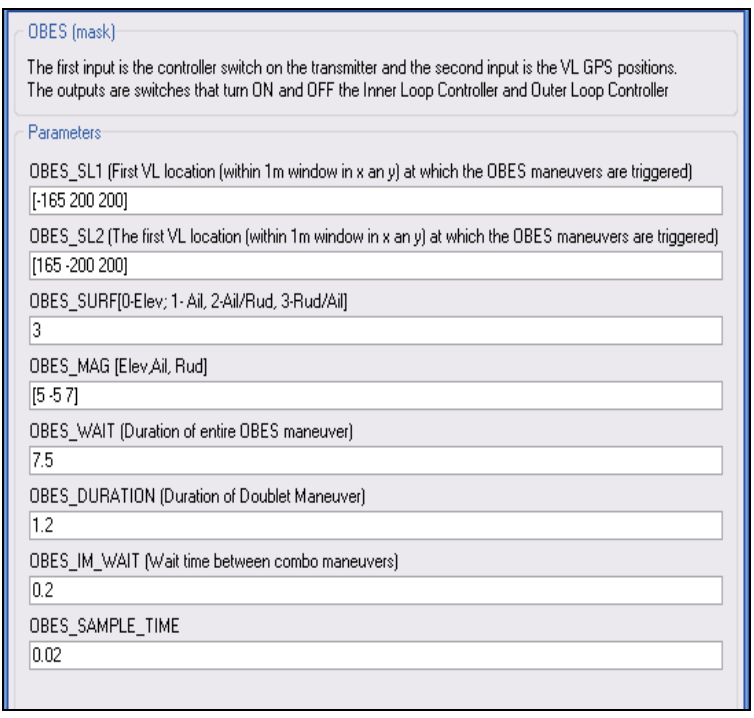

Figure 33: GUI Mask for OBES Subsystem

Table 9 summarizes the maneuvers that were used for off-line PID analysis for the short period mode, and Table 11 summarizes the maneuvers used for off-line analysis of the Dutch roll and roll modes. Tables 10 and 12 highlight the average, median, and standard deviation of the damping ratios and natural frequencies of the selected modes.

A total of 34 maneuvers were performed for the short period analysis. Table 9 highlights the flight data, maneuver performed (either manual or OBES), time segment from that flight, eigenvalues of the short period mode, the short period damping ratio, and the short period natural frequency. Table 10 shows the statistical analysis of the short period mode, specifically the 
average, median, and standard deviation of the damping ratio and natural frequency. Selected outliers were removed and the analysis was repeated for these statistical measures and reported in Table 10.

Table 11 shows the 28 maneuvers performed to identify the Dutch roll and roll modes. Table 11 also shows the flight data, maneuver performed, time segment from that flight, eigenvalues of the Dutch roll and roll modes, and the damping ratio and natural frequency of the Dutch roll. Table 12 shows the statistical analysis of the Dutch roll and roll modes. The average, median, and standard deviation of the roll time constant and the Dutch roll damping ratio and natural frequency were evaluated. Again, major outliers were removed from the data and these statistical results were also reported in Table 12 . 
Table 9: Stabilator Maneuvers and Resulting Short Period Properties of WVU YF-22

\begin{tabular}{|c|c|c|c|c|c|c|}
\hline \multicolumn{7}{|c|}{ Longitudinal PID Results } \\
\hline Flight Date & Maneuver & $\begin{array}{c}\text { Time Segment } \\
\text { (sec) }\end{array}$ & $\begin{array}{l}\text { Eigenvalues } \\
\text { (Short Period) }\end{array}$ & $\begin{array}{c}\text { Damping } \\
\text { Ratio }\end{array}$ & $\begin{array}{c}\text { Natural } \\
\text { Frequency } \\
\text { (rps) }\end{array}$ & Number \\
\hline $9 / 16 / 2008$ & Stabilator & $224-228.5$ & $-7.13 \pm 8.55 \mathrm{i}$ & 0.64 & 11.13 & 1 \\
\hline $9 / 16 / 2008$ & Stabilator & $426-428.74$ & $-6.06 \pm 6.80 \mathrm{i}$ & 0.67 & 9.10 & 2 \\
\hline $10 / 18 / 2008$ & Stabilator - OBES & $263-269$ & $-5.60 \pm 6.60 \mathrm{i}$ & 0.65 & 8.66 & 3 \\
\hline $10 / 18 / 2008$ & Stabilator - OBES & $293-299$ & $-5.05 \pm 4.31 \mathrm{i}$ & 0.76 & 6.64 & 4 \\
\hline $10 / 18 / 2008$ & Stabilator - OBES & $324-330$ & $-6.22 \pm 5.26 \mathrm{i}$ & 0.76 & 8.15 & 5 \\
\hline $10 / 18 / 2008$ & Stabilator - OBES & $354-360$ & $-5.20 \pm 5.46 \mathrm{i}$ & 0.69 & 7.54 & 6 \\
\hline $10 / 18 / 2008$ & Stabilator - OBES & $385-391$ & $-5.51 \pm 4.44 \mathrm{i}$ & 0.79 & 7.08 & 7 \\
\hline $10 / 18 / 2008$ & Stabilator - OBES & $415-421$ & $-6.19 \pm 2.72 \mathrm{i}$ & 0.92 & 6.76 & 8 \\
\hline $10 / 18 / 2008$ & Stabilator - OBES & $446-452$ & $-5.89 \pm 4.38 \mathrm{i}$ & 0.80 & 7.34 & 9 \\
\hline $10 / 18 / 2008$ & Stabilator & $478.5-484$ & $-6.01 \pm 6.30 \mathrm{i}$ & 0.69 & 8.71 & 10 \\
\hline $10 / 18 / 2008$ & Stabilator & $506-511$ & $-7.03 \pm 7.64 \mathrm{i}$ & 0.68 & 10.38 & 11 \\
\hline $11 / 1 / 2008$ & Stabilator & $569-573$ & $-6.57 \pm 7.29 \mathrm{i}$ & 0.67 & 9.81 & 12 \\
\hline $10 / 11 / 2008$ & Stabilator - OBES & $333.22-338$ & $-6.40 \pm 7.19 \mathrm{i}$ & 0.66 & 9.63 & 13 \\
\hline $10 / 11 / 2008$ & Stabilator - OBES & $357.2-360$ & $-4.92 \pm 4.11 \mathrm{i}$ & 0.77 & 6.41 & 14 \\
\hline $10 / 11 / 2008$ & Stabilator - OBES & $380.5-386$ & $-6.52 \pm 6.83 \mathrm{i}$ & 0.69 & 9.45 & 15 \\
\hline $10 / 11 / 2008$ & Stabilator - OBES & 404.9-409.5 & $-5.64 \pm 5.45 \mathrm{i}$ & 0.72 & 7.84 & 16 \\
\hline $10 / 11 / 2008$ & Stabilator - OBES & $428.5-433.5$ & $-6.66 \pm 6.92 \mathrm{i}$ & 0.69 & 9.61 & 17 \\
\hline $10 / 11 / 2008$ & Stabilator - OBES & $452.25-457.5$ & $-5.39 \pm 4.29 \mathrm{i}$ & 0.78 & 6.89 & 18 \\
\hline $10 / 11 / 2008$ & Stabilator - OBES & $476-481.25$ & $-6.67 \pm 6.96 \mathrm{i}$ & 0.69 & 9.64 & 19 \\
\hline $5 / 22 / 2009$ & Stabilator & $362-368$ & $-6.15 \pm 6.36 \mathrm{i}$ & 0.70 & 8.84 & 20 \\
\hline $5 / 22 / 2009$ & Stabilator & $398-405$ & $-4.96 \pm 6.18 \mathrm{i}$ & 0.63 & 7.93 & 21 \\
\hline $5 / 22 / 2009$ & Stabilator & $264-270$ & $-5.92 \pm 6.51 \mathrm{i}$ & 0.67 & 8.80 & 22 \\
\hline $5 / 22 / 2009$ & Stabilator & $295-302$ & $-5.71 \pm 6.39 \mathrm{i}$ & 0.67 & 8.57 & 23 \\
\hline $5 / 22 / 2009$ & Stabilator & $489-495$ & $-6.71 \pm 6.56 \mathrm{i}$ & 0.71 & 9.38 & 24 \\
\hline $5 / 22 / 2009$ & Stabilator & $513-519$ & $-6.22 \pm 6.80 \mathrm{i}$ & 0.67 & 9.22 & 25 \\
\hline $6 / 9 / 2010$ & Stabilator & $265.5-274$ & $-4.79 \pm 6.68 \mathrm{i}$ & 0.58 & 8.18 & 26 \\
\hline $6 / 9 / 2010$ & Stabilator & $286-290.5$ & $-5.41 \pm 6.92 \mathrm{i}$ & 0.62 & 8.78 & 27 \\
\hline $6 / 9 / 2010$ & Stabilator & $390-394$ & $-5.19 \pm 8.04 \mathrm{i}$ & 0.54 & 9.57 & 28 \\
\hline $6 / 9 / 2010$ & Stabilator & $410-416$ & $-6.58 \pm 8.90 \mathrm{i}$ & 0.59 & 11.07 & 29 \\
\hline $4 / 18 / 2010$ & Stabilator - 1123 & $544.5-552$ & $-4.15 \pm 7.80 \mathrm{i}$ & 0.47 & 8.84 & 30 \\
\hline $4 / 18 / 2010$ & Stabilator - 1123 & $597-604$ & $-4.66 \pm 7.15 i$ & 0.55 & 8.53 & 31 \\
\hline $4 / 18 / 2010$ & Stabilator - 1123 & $441-448$ & $-5.25 \pm 6.10 \mathrm{i}$ & 0.65 & 8.05 & 32 \\
\hline $4 / 18 / 2010$ & Stabilator - 1123 & $470-477$ & $-5.33 \pm 5.73 \mathrm{i}$ & 0.68 & 7.83 & 33 \\
\hline $4 / 18 / 2010$ & Stabilator - 1123 & $499-505.4$ & $-5.09 \pm 5.53 \mathrm{i}$ & 0.68 & 7.52 & 34 \\
\hline
\end{tabular}


Table 10: Short Period Damping Ratio and Natural Frequency Statistical Analysis

\begin{tabular}{|c|c|c|c|}
\hline & & Damping Ratio & Natural Frequency (rps) \\
\hline & Average & 0.68 & 8.58 \\
\hline & Median & 0.68 & 8.68 \\
\hline & Standard Deviation & 0.08 & 1.19 \\
\hline & Average & 0.68 & 8.55 \\
\cline { 2 - 4 } & Median & 0.68 & 8.68 \\
\cline { 2 - 4 } $\begin{array}{c}\text { Removed Major } \\
\text { Outliers }\end{array}$ & Standard Deviation & 0.05 & 0.86 \\
\hline
\end{tabular}

Table 11: Aileron and Rudder Maneuvers and Resulting Dutch Roll and Roll Properties of WVU YF-22

\begin{tabular}{|c|c|c|c|c|c|c|c|}
\hline \multicolumn{8}{|c|}{ Lateral-Directional PID Results } \\
\hline Flight Date & Maneuver & \begin{tabular}{|c|}
$\begin{array}{c}\text { Time Segment } \\
(\mathrm{sec})\end{array}$ \\
\end{tabular} & $\begin{array}{l}\text { Eigenvalues } \\
\text { (Dutch Roll) }\end{array}$ & $\begin{array}{c}\text { Eigenvalues } \\
(\text { Roll) }\end{array}$ & $\begin{array}{c}\text { Damping } \\
\text { Ratio }\end{array}$ & \begin{tabular}{|c|} 
Natural \\
Frequency (rps) \\
\end{tabular} & Number \\
\hline $9 / 16 / 2008$ & Rudder/Aileron & $444-450$ & $-1.09 \pm 5.11 \mathrm{i}$ & -11.86 & 0.21 & 5.22 & 1 \\
\hline $10 / 18 / 2008$ & Rudder/Aileron - OBES & $324-331$ & $-0.42 \pm 5.89 \mathrm{i}$ & -7.00 & 0.07 & 5.91 & 2 \\
\hline $10 / 18 / 2008$ & Rudder/Aileron - OBES & $324-330.5$ & $-0.67 \pm 5.66 \mathrm{i}$ & -7.85 & 0.12 & 5.70 & 3 \\
\hline $10 / 18 / 2008$ & Rudder/Aileron - OBES & $354-361$ & $-0.76 \pm 5.28 \mathrm{i}$ & -17.17 & 0.14 & 5.34 & 4 \\
\hline $10 / 18 / 2008$ & Rudder/Aileron - OBES & $384.5-391.5$ & $-1.08 \pm 5.88 \mathrm{i}$ & -14.51 & 0.18 & 5.98 & 5 \\
\hline $10 / 18 / 2008$ & Rudder/Aileron - OBES & $415-422$ & $-0.86 \pm 5.39 \mathrm{i}$ & -5.27 & 0.16 & 5.46 & 6 \\
\hline $10 / 18 / 2008$ & Rudder/Aileron - OBES & $445.5-452$ & $-1.13 \pm 6.13 i$ & -10.02 & 0.18 & 6.24 & 7 \\
\hline $10 / 18 / 2008$ & Rudder/Aileron - OBES & $476-483$ & $-0.76 \pm 5.08 \mathrm{i}$ & -8.012 & 0.15 & 5.13 & 8 \\
\hline $10 / 18 / 2008$ & Rudder/Aileron & $512-520$ & $-1.00 \pm 5.69 \mathrm{i}$ & -11.57 & 0.17 & 5.78 & 9 \\
\hline $10 / 18 / 2008$ & Rudder/Aileron & $539-547$ & $-0.57 \pm 5.51 \mathrm{i}$ & -14.22 & 0.10 & 5.54 & 10 \\
\hline $5 / 22 / 2009$ & Aileron & $427-433$ & - & -12.47 & - & - & 11 \\
\hline $5 / 22 / 2009$ & Rudder/Aileron & $492-501$ & $-1.07 \pm 5.77 \mathrm{i}$ & -11.57 & 0.18 & 5.86 & 12 \\
\hline $5 / 22 / 2009$ & Rudder/Aileron & $531-540$ & $-1.05 \pm 5.55 \mathrm{i}$ & -11.59 & 0.19 & 5.65 & 13 \\
\hline $5 / 22 / 2009$ & Aileron & $324-332$ & - & -11.68 & - & - & 14 \\
\hline $5 / 22 / 2009$ & Aileron & $353-360$ & - & -12.40 & - & - & 15 \\
\hline $5 / 22 / 2009$ & Rudder/Aileron & $380-390$ & $-1.03 \pm 5.62 \mathrm{i}$ & -13.12 & 0.18 & 5.62 & 16 \\
\hline $5 / 22 / 2009$ & Rudder/Aileron & $410-419$ & $-1.17 \pm 5.97 \mathrm{i}$ & -12.93 & 0.19 & 6.09 & 17 \\
\hline $5 / 22 / 2009$ & Rudder/Aileron & $410-417$ & $-1.17 \pm 5.97 \mathrm{i}$ & -13.12 & 0.19 & 6.09 & 18 \\
\hline $5 / 22 / 2009$ & Rudder/Aileron & $435.6-444$ & $-1.09 \pm 5.75 \mathrm{i}$ & -14.06 & 0.19 & 5.85 & 19 \\
\hline $5 / 22 / 2009$ & Rudder/Aileron & $463-470.5$ & $-1.08 \pm 6.04 \mathrm{i}$ & -13.34 & 0.18 & 6.14 & 20 \\
\hline $5 / 22 / 2009$ & Aileron & $538.5-546$ & - & -11.18 & - & - & 21 \\
\hline $6 / 19 / 2010$ & Aileron & $306-310.5$ & - & -7.01 & - & - & 22 \\
\hline $6 / 19 / 2010$ & Aileron & $326-330$ & - & -10.82 & - & - & 23 \\
\hline $6 / 19 / 2010$ & Rudder/Aileron & $347.6-356$ & $-0.94 \pm 5.73 \mathrm{i}$ & -8.19 & 0.16 & 5.73 & 24 \\
\hline $6 / 19 / 2010$ & Rudder/Aileron & $369-374$ & $-1.03 \pm 5.96 \mathrm{i}$ & -9.48 & 0.17 & 6.05 & 25 \\
\hline $6 / 19 / 2010$ & Rudder/Aileron & $432.2-437.2$ & $-0.96 \pm 5.89 \mathrm{i}$ & -9.36 & 0.16 & 5.97 & 26 \\
\hline $6 / 19 / 2010$ & Rudder/Aileron & $455-459$ & $-1.05 \pm 6.48 \mathrm{i}$ & -10.17 & 0.16 & 6.57 & 27 \\
\hline $6 / 19 / 2010$ & Aileron & $474-478$ & - & -10.15 & - & - & 28 \\
\hline
\end{tabular}


Table 12: Dutch Roll Damping and Natural Frequency and Roll Mode Time Constant Statistical Analysis

\begin{tabular}{|c|c|c|c|c|}
\hline & & $\begin{array}{c}\text { Roll Time } \\
\text { Constant (sec) }\end{array}$ & $\begin{array}{c}\text { Damping } \\
\text { Ratio }\end{array}$ & $\begin{array}{c}\text { Natural Frequency } \\
\text { (rps) }\end{array}$ \\
\hline & Average & 11.08 & 0.16 & 5.79 \\
\hline & Median & 11.57 & 0.17 & 5.81 \\
\hline & Standard Deviation & 2.68 & 0.03 & 0.35 \\
\hline \multirow{3}{*}{$\begin{array}{c}\text { Removed } \\
\text { Major } \\
\text { Outliers } \\
\end{array}$} & Average & 11.13 & 0.16 & 5.76 \\
\hline & Median & 11.57 & 0.17 & 5.78 \\
\hline & Standard Deviation & 1.80 & 0.03 & 0.31 \\
\hline
\end{tabular}

In addition to the longitudinal and lateral-directional states identified using the System Identification Toolbox, $\dot{V}, \dot{\theta}$, and $\dot{\phi}$ were also derived for a more complete linear model. While $\dot{\theta}$ and $\dot{\phi}$ were identified as the pitch rate and roll rate, respectively, $\dot{V}$ was derived by using contributions from the angle of attack, stabilator deflection, and aircraft velocity. The resulting continuous-time nominal longitudinal and lateral-directional linear state space models were identified as:

$$
\begin{gathered}
{\left[\begin{array}{c}
\dot{V} \\
\dot{\alpha} \\
\dot{q} \\
\dot{\theta}
\end{array}\right]=\left[\begin{array}{cccc}
-0.284 & -23.096 & 0 & -0.171 \\
0 & -3.767 & 1.126 & 0 \\
0 & -39.651 & -7.656 & 0 \\
0 & 0 & 1 & 0
\end{array}\right]\left[\begin{array}{c}
V \\
\alpha \\
q \\
\theta
\end{array}\right]+\left[\begin{array}{c}
-20.168 \\
2.087 \\
-74.233 \\
0
\end{array}\right] i_{H}} \\
{\left[\begin{array}{c}
\dot{\beta} \\
\dot{p} \\
\dot{r} \\
\dot{\phi}
\end{array}\right]=\left[\begin{array}{cccc}
-0.019 & 0.040 & -1.199 & 0 \\
-99.224 & -13.177 & 3.223 & 0 \\
23.060 & -0.488 & -1.982 & 0 \\
0 & 1 & 0 & 0
\end{array}\right]\left[\begin{array}{c}
\beta \\
p \\
r \\
\phi
\end{array}\right]+\left[\begin{array}{cc}
0.049 & -0.460 \\
-184.269 & 32.135 \\
-5.018 & -28.090 \\
0 & 0
\end{array}\right]\left[\begin{array}{l}
\delta_{a} \\
\delta_{r}
\end{array}\right]}
\end{gathered}
$$

The corresponding eigenvalues along with the damping, natural frequency, and time constant values for the dynamic modes are listed in Table 13.

Table 13: Eigenvalues, Damping, and Natural Frequencies of WVU YF-22 Aircraft (Nominal)

\begin{tabular}{|c|c|c|c|}
\hline Dynamic Mode & Eigenvalues & Damping & $\begin{array}{c}\text { Natural Frequency } \\
\text { (rad/sec) }\end{array}$ \\
\hline Short Period & $-5.71 \pm 6.39 \mathrm{i}$ & 0.67 & 8.57 \\
\hline Dutch Roll & $-1.03 \pm 5.62 \mathrm{i}$ & 0.18 & 5.71 \\
\hline Roll & -13.12 & - & - \\
\hline
\end{tabular}

The state space models represented in Equations 5.1 and 5.2 were utilized a preliminary step for control law design as well as a starting point for the stability and control derivative identification process. These state space parameters were converted to baseline stability and control derivatives using the geometric and inertial properties of the aircraft. These estimates 
served as the initial values for optimization of the stability and control derivatives, a process that is discussed in Section 5.1.2.

A second step was performed using an optimization scheme to improve the estimates of the state space models in Equations 5.1 and 5.2 for control law design. A output-error optimization scheme was adapted from the System Identification Programs for AirCraft (SIDPAC) ${ }^{\circledR}$ developed by Eugene Morelli ${ }^{51}$, to optimize the state space parameters for the WVU YF-22 for control law design. Segments of flight data were selected based on the input maneuver, as several data sets were "meshed" together as if they were recorded consecutively. Figures 34 and 35 show flight data segments that were used in the optimization process. These inputs include stabilator doublets for the longitudinal optimization and rudder and aileron doublet combinations for the lateral-directional optimization.

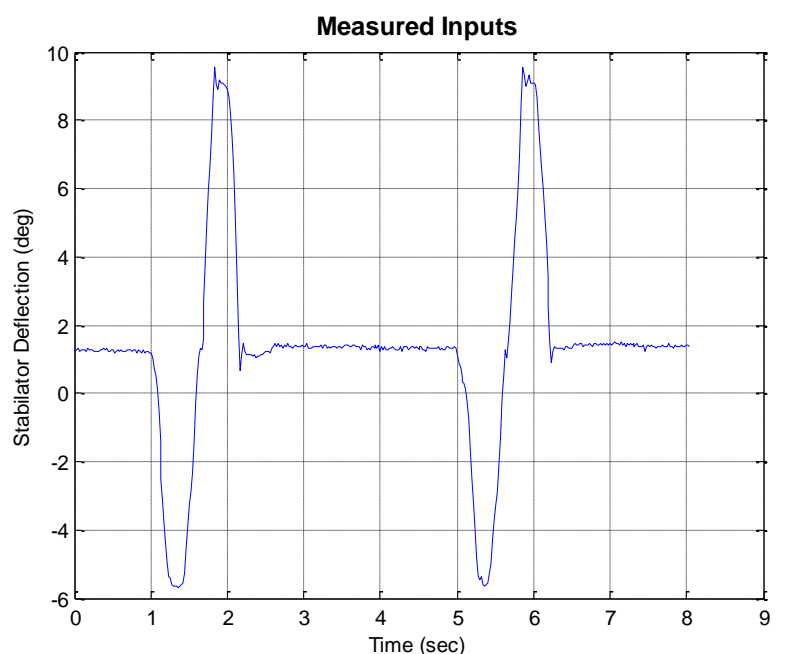

Figure 34: Stabilator Doublets used for Optimization
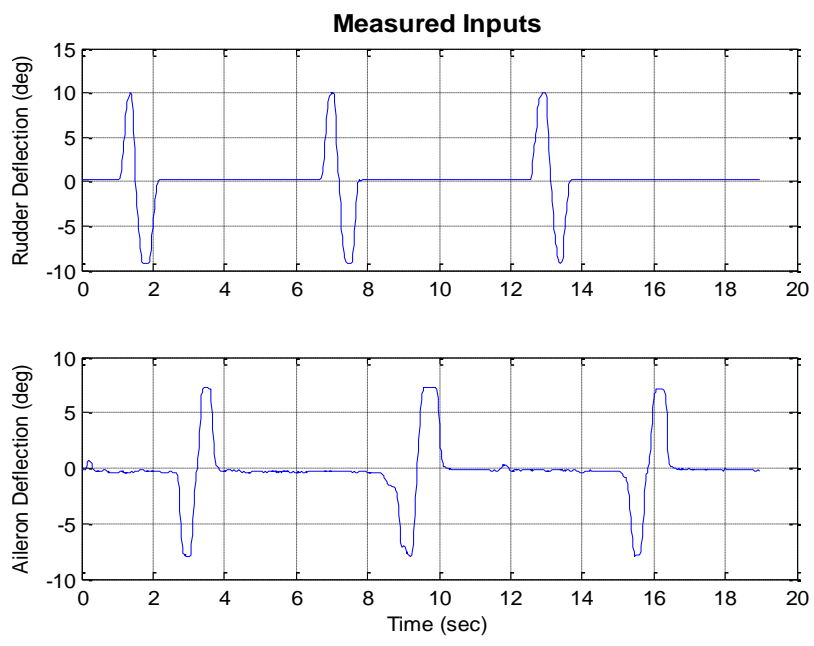

Figure 35: Rudder and Aileron Doublets used for Optimization

Through this time-domain optimization process, the output-error maximum likelihood estimate of the state space model parameters were computed independently for the longitudinal and lateral-directional dynamics. The modified Newton-Raphson was used as the optimizing algorithm to decrease the output value of the cost function. For the longitudinal optimization the output states under consideration were the angle of attack, pitch rate, and linear acceleration, while for the lateral-directional optimization the output states were sideslip, roll rate, yaw rate, bank angle, and linear acceleration. Figures 36 and 37 show the optimized output versus the actual measured output for sets of flight data implemented for optimization. 

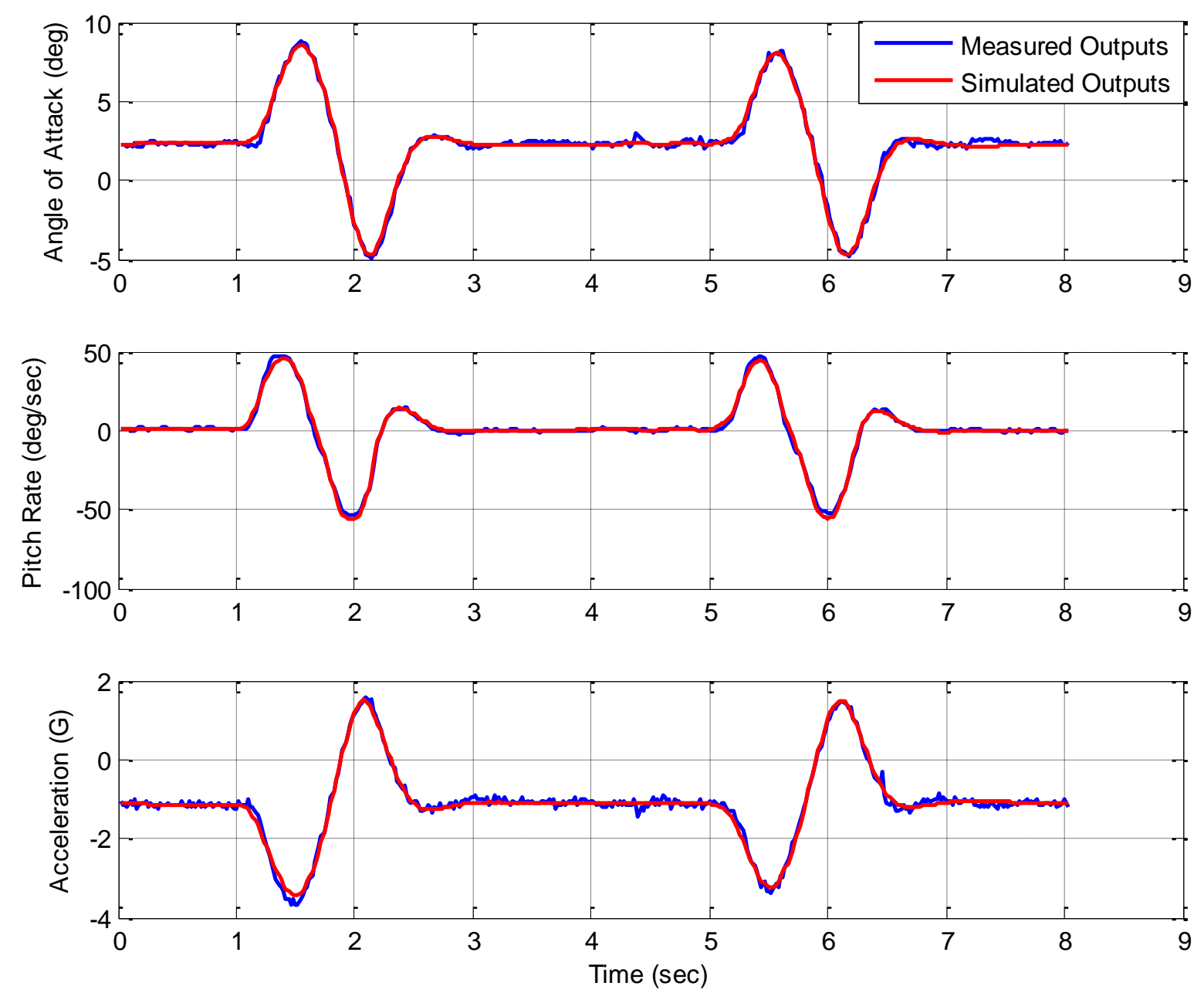

Figure 36: Measured versus Optimized Longitudinal Simulated Output 

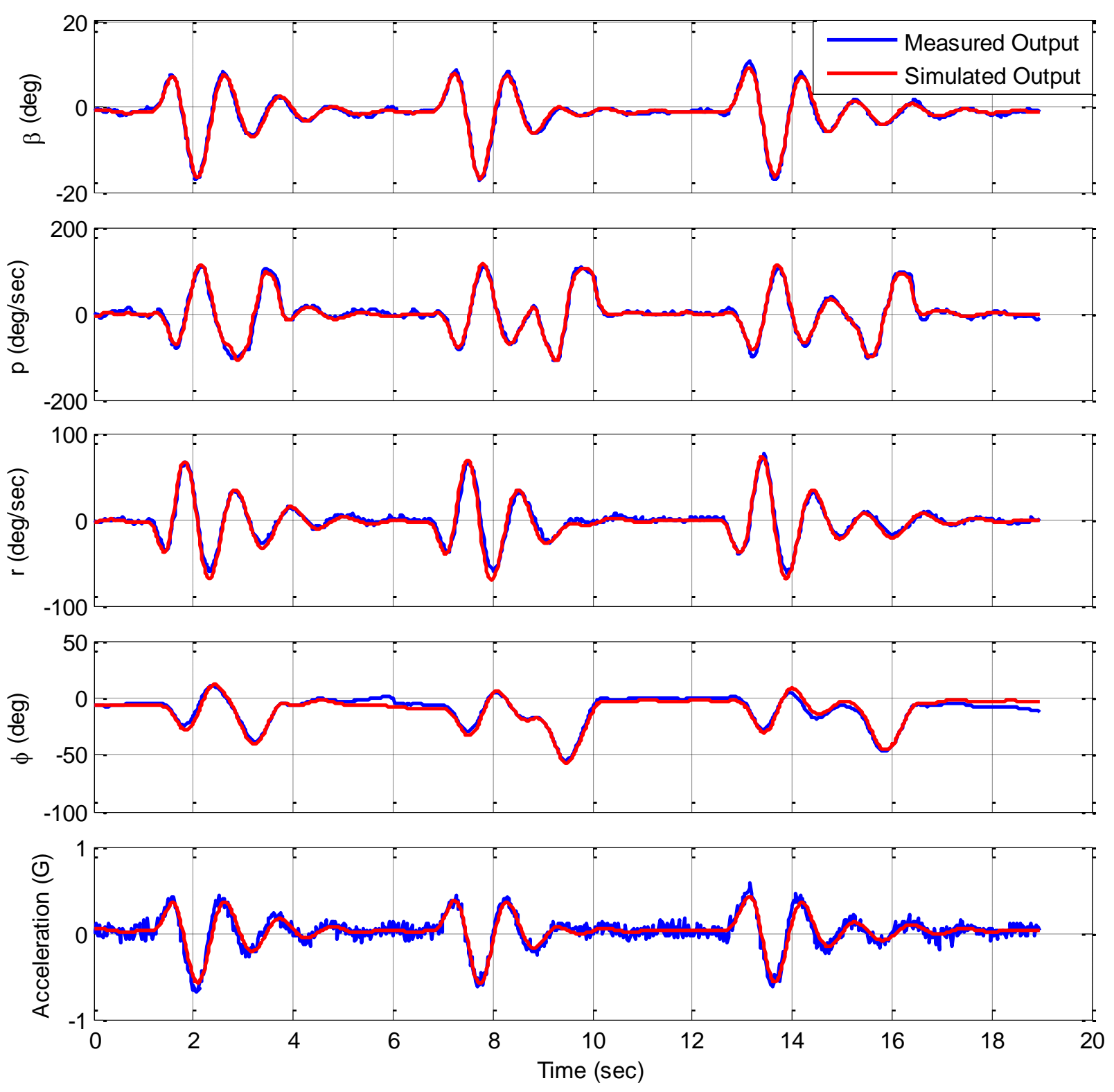

Figure 37: Measured versus Optimized Lateral-Directional Simulated Output 
The optimized linear state space model is represented in Equations 5.3 and 5.4.

$$
\begin{gathered}
{\left[\begin{array}{c}
\dot{V} \\
\dot{\alpha} \\
\dot{q} \\
\dot{\theta}
\end{array}\right]=\left[\begin{array}{cccc}
-0.284 & -23.096 & 0 & -0.171 \\
0 & -3.991 & 0.916 & 0 \\
0 & -35.922 & -6.539 & 0 \\
0 & 0 & 1 & 0
\end{array}\right]\left[\begin{array}{l}
V \\
\alpha \\
q \\
\theta
\end{array}\right]+\left[\begin{array}{c}
-20.168 \\
0.675 \\
-67.420 \\
0
\end{array}\right] i_{H}} \\
{\left[\begin{array}{c}
\dot{\beta} \\
\dot{p} \\
\dot{r} \\
\dot{\phi}
\end{array}\right]=\left[\begin{array}{cccc}
0.525 & 0.052 & -0.999 & 0 \\
-107.780 & -12.482 & 3.241 & 0 \\
33.705 & -0.488 & -2.553 & 0 \\
0 & 1 & 0 & 0
\end{array}\right]\left[\begin{array}{l}
\beta \\
p \\
r \\
\phi
\end{array}\right]+\left[\begin{array}{cc}
0.240 & -0.497 \\
-170.372 & 25.552 \\
-1.466 & -29.170 \\
0 & 0
\end{array}\right]\left[\begin{array}{l}
\delta_{a} \\
\delta_{r}
\end{array}\right]}
\end{gathered}
$$

The corresponding eigenvalues along with the damping, natural frequency, and time constant values for the optimized dynamic modes are listed in Table 14.

Table 14: Optimized Eigenvalues, Damping, and Natural Frequencies of WVU YF-22 Aircraft (Nominal)

\begin{tabular}{|c|c|c|c|}
\hline Dynamic Mode & Eigenvalues & Damping & $\begin{array}{c}\text { Natural Frequency } \\
\text { (rad/sec) }\end{array}$ \\
\hline Short Period & $-5.27 \pm 5.59 \mathrm{i}$ & 0.69 & 7.68 \\
\hline Dutch Roll & $-1.12 \pm 5.98 \mathrm{i}$ & 0.18 & 6.09 \\
\hline Roll & -12.27 & - & - \\
\hline
\end{tabular}

These optimized state space parameters were also converted to baseline stability and control derivatives using the geometric and inertial properties of the aircraft. These estimates served as initial values for another optimization process for the stability and control derivatives, which is discussed in Section 5.1.2. A confidence interval analysis was also conducted on the optimized parameters shown in Equations 5.3 and 5.4. A full discussion of these parameter estimates is in Section 5.1.6.

\subsubsection{CoDICE Modeling Results}

Parameter identification flight tests were conducted during 2008 with the WVU YF-22 to estimate a mathematical model of the aircraft under primary control surface failure conditions. Specific maneuvers were performed with the either a stabilator or aileron locked at trim, as commanded by the onboard computer. Doublet maneuvers were performed on the healthy stabilator during stabilator failures, and rudder/aileron doublet combinations and aileron doublets were performed on the healthy aileron during aileron failure conditions. These maneuvers were performed both manually by a pilot and autonomously by on on-board excitation system. Rudder 
failures were not examined in this case because the YF-22 did not have individual control capability for each rudder. During the periods of simulated control surface failures, the crosscoupling of the longitudinal and lateral-directional dynamics was modeled. During the stabilator failures, the individual effects of the healthy stabilator on the sideslip, roll rate, and yaw rate were examined. Conversely, during aileron failures the individual effects of the healthy aileron on the angle off attack and pitch rate were observed.

As previously mentioned, there were no changes to the aerodynamic properties since there was no damage to the aircraft. Since the only simulated failures were locked actuators, the control $(A)$ matrix remained unchanged from the nominal model. The input $(B)$ matrix, however, was divided into six individual control surface inputs affecting both longitudinal and lateral directional states. Based on the assumption that the aerodynamic properties of the aircraft remain unchanged, the optimized nominal state space model was implemented with split control surface contributions in the input matrix. Because the control surface pairs are assumed to be symmetric, the longitudinal contributions from the stabilator pair and the lateral-directional contributions form the aileron and rudder pairs may be halved. Thus, the resulting state space representation is shown in Equation 5.5. The terms in Equation 5.5 that are represented with an $X$ are the crosscoupled terms that required identification from the sets of flight data with induced control surface failures. 


$$
\begin{aligned}
{\left[\begin{array}{c}
\dot{V} \\
\dot{\alpha} \\
\dot{q} \\
\dot{\theta} \\
\dot{\beta} \\
\dot{p} \\
\dot{r} \\
\dot{\phi}
\end{array}\right]=\left[\begin{array}{cccccccc}
-0.284 & -23.096 & 0 & -0.171 & 0 & 0 & 0 & 0 \\
0 & -3.991 & 0.916 & 0 & 0 & 0 & 0 & 0 \\
0 & -35.922 & -6.539 & 0 & 0 & 0 & 0 & 0 \\
0 & 0 & 1 & 0 & 0 & 0 & 0 & 0 \\
0 & 0 & 0 & 0 & 0.525 & 0.052 & -0.999 & 0 \\
0 & 0 & 0 & 0 & -107.780 & -12.482 & 3.241 & 0 \\
0 & 0 & 0 & 0 & 33.705 & -0.488 & -2.553 & 0 \\
0 & 0 & 0 & 0 & 0 & 1 & 0 & 0
\end{array}\right]\left[\begin{array}{l}
V \\
\alpha \\
q \\
\theta \\
\beta \\
p \\
r \\
\phi
\end{array}\right] } \\
+\left[\begin{array}{ccccccc}
-10.084 & -10.084 & 0 & 0 & 0 & 0 \\
0.338 & 0.338 & \mathbf{X} & \mathbf{X} & 0 & 0 \\
-33.710 & -33.710 & \mathbf{X} & \mathbf{X} & 0 & 0 \\
0 & 0 & 0 & 0 & 0 & 0 \\
\mathbf{X} & \mathbf{X} & -0.240 & 0.240 & -0.248 & -0.248 \\
\mathbf{X} & \mathbf{X} & 85.186 & -85.186 & 12.776 & 12.776 \\
\mathbf{X} & \mathbf{X} & 0.733 & -0.733 & -14.585 & -14.585 \\
0 & 0 & 0 & 0 & 0 & 0
\end{array}\right]\left[\begin{array}{l}
i_{H_{L}} \\
i_{H_{R}} \\
\delta_{a_{L}} \\
\delta_{a_{R}} \\
\delta_{r_{L}} \\
\delta_{r_{R}}
\end{array}\right]
\end{aligned}
$$

A frequency-based method was used to identify the cross-coupled terms during control surface maneuvers with induced failures. The FTR method was implemented using Simulink ${ }^{\circledR}$ and the Parameter Identification Library ${ }^{82}$. This identification process was implemented using sets of measured flight data where individual control surface deflections were performed. Through this means, the input was the individual control surface deflection and the output states observed were those representing the cross-coupled terms. Specifically, during an individual stabilator deflection the sideslip, roll rate, and yaw rate were observed. Conversely, during an individual aileron deflection, the angle of attack and pitch rate were observed. The control surface input as well as the measured output states were utilized as described in Section 4.3.3, with the coupled $A$ matrix being required for preprocessing the data for the FTR scheme. Figure 38 shows the general layout of the FTR scheme used for the identification of the cross-coupled input matrix terms. 


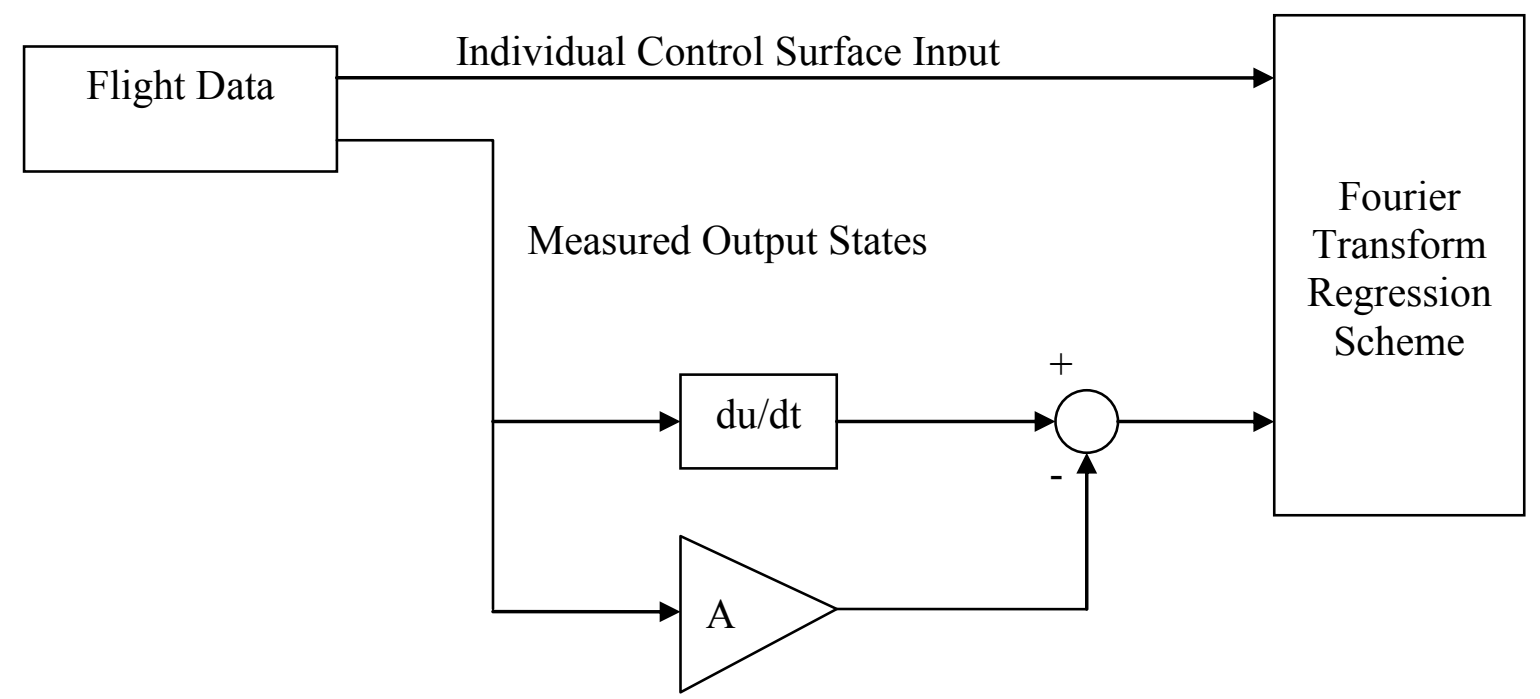

Figure 38: Cross-Coupled Input Matrix Term Identification Process

Each maneuver performed in flight was simulated through this scheme to estimate the crosscoupled input matrix terms. Figures 39 and 40 show sample data segments that were used for the identification process. For the individual stabilator doublets, the roll rate was the primary focus for identification since the sideslip and yaw rates were minutely affected. For the individual aileron doublets, both the angle of attack and pitch rate were significantly affected. 

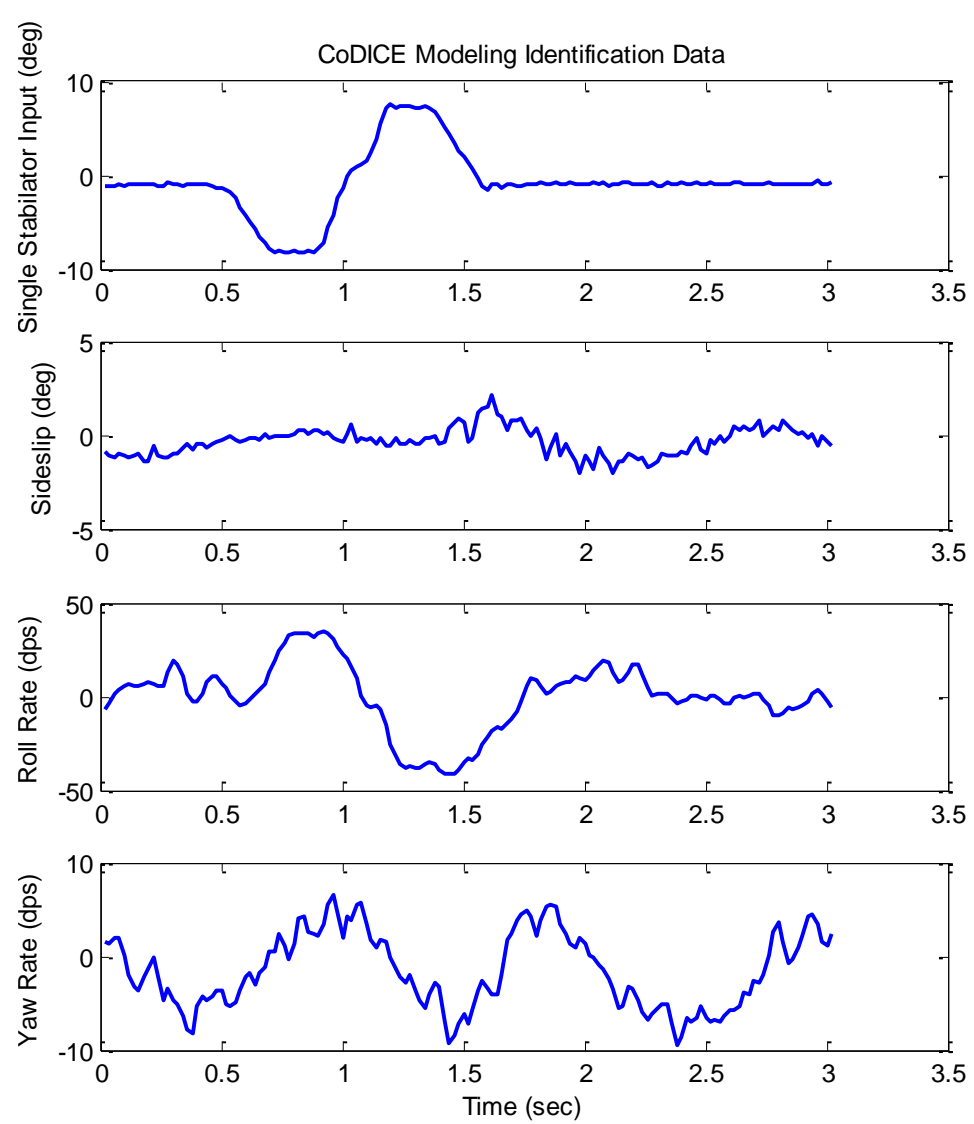

Figure 39: Individual Stabilator Effects on Lateral-Directional States
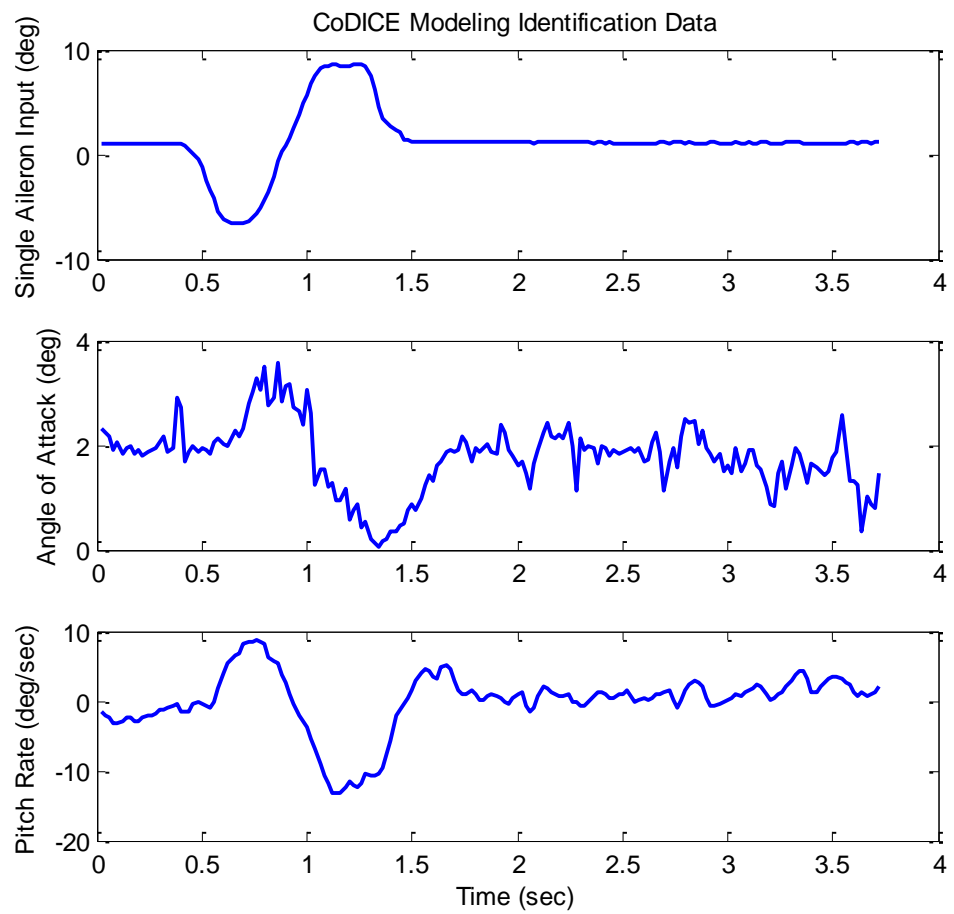

Figure 40: Individual Aileron Effects on Longitudinal States 
As each of eight individual stabilator maneuvers and nine individual aileron maneuvers were run through the FTR scheme, the cross-coupled input matrix components were estimated. The estimated values for the individual stabilator effects on the lateral-directional states are shown in Table 15.

Table 15: Identification Results for Individual Stabilator Deflections

\begin{tabular}{|c|c|l|c|c|c|}
\hline Number & Date & Maneuver & $\mathbf{B}(\mathbf{5 , 2})$ & $\mathbf{B}(\mathbf{6 , 2})$ & $\mathbf{B}(\mathbf{7 , 2})$ \\
\hline 1 & $9 / 16 / 2008$ & Stabilator & 0.57 & -55.50 & -0.57 \\
\hline 2 & $9 / 16 / 2008$ & Stabilator & 0.26 & -58.50 & -0.62 \\
\hline 3 & $9 / 16 / 2008$ & Stabilator & 0.30 & -49.50 & -0.49 \\
\hline 4 & $9 / 16 / 2008$ & Stabilator & 0.23 & -56.25 & -0.48 \\
\hline 5 & $9 / 16 / 2008$ & Stabilator & 0.43 & -55.00 & -0.55 \\
\hline 6 & $9 / 16 / 2008$ & Stabilator & 0.40 & -50.00 & -0.52 \\
\hline 7 & $9 / 16 / 2008$ & Stabilator & 0.27 & -62.50 & -0.50 \\
\hline 8 & $9 / 16 / 2008$ & Stabilator & 0.32 & -54.00 & -0.52 \\
\hline
\end{tabular}

Table 16 provides a statistical analysis of the average, median, and standard deviation of the estimated parameters.

Table 16: Statistical Analysis of Identification Method Results for Individual Stabilator Doublets

\begin{tabular}{|c|c|c|c|}
\hline Statistic & $\mathbf{B}(\mathbf{5 , 2})$ & $\mathbf{B}(\mathbf{6 , 2})$ & $\mathbf{B}(\mathbf{7 , 2})$ \\
\hline FTR Average & 0.33 & -54.88 & -0.53 \\
\hline FTR Median & 0.31 & -55.25 & -0.52 \\
\hline FTR Standard Deviation & 0.07 & 2.83 & 0.03 \\
\hline
\end{tabular}

Table 17 provides the input matrix estimation results from the FTR scheme for individual aileron deflections and their effect on the longitudinal states.

Table 17: Identification Results for Individual Aileron Deflections

\begin{tabular}{|c|c|c|c|c|}
\hline Number & Date & Maneuver & $\mathbf{B}(\mathbf{2 , 3})$ & $\mathbf{B}(\mathbf{3 , 3})$ \\
\hline 1 & $9 / 16 / 2008$ & Aileron & 0.22 & -2.50 \\
\hline 2 & $9 / 16 / 2008$ & Aileron & 0.10 & -2.70 \\
\hline 3 & $9 / 16 / 2008$ & Aileron & 0.15 & -1.43 \\
\hline 4 & $9 / 16 / 2008$ & Aileron & 0.13 & -2.40 \\
\hline 5 & $9 / 16 / 2008$ & Aileron & 0.14 & -2.90 \\
\hline 6 & $9 / 16 / 2008$ & Aileron & 0.21 & -3.25 \\
\hline 7 & $9 / 16 / 2008$ & Aileron & 0.11 & -3.00 \\
\hline 8 & $9 / 16 / 2008$ & Aileron & 0.20 & -3.50 \\
\hline 9 & $9 / 16 / 2008$ & Aileron & 0.09 & -2.30 \\
\hline
\end{tabular}

Table 18 provides a statistical analysis of the average, median, and standard deviation of the estimated parameters. 
Table 18: Statistical Analysis of Identification Method Results for Individual Aileron Doublets

\begin{tabular}{|c|c|c|}
\hline Statistic & $\mathbf{B}(\mathbf{2 , 3})$ & $\mathbf{B}(\mathbf{3 , 3})$ \\
\hline FTR Average & 0.15 & -2.72 \\
\hline FTR Median & 0.14 & -2.70 \\
\hline FTR Standard Deviation & 0.04 & 0.35 \\
\hline
\end{tabular}

Equation 5.6 shows the CoDICE state space model of the WVU YF-22 aircraft that was used for control law design.

$$
\begin{aligned}
{\left[\begin{array}{c}
\dot{V} \\
\dot{\alpha} \\
\dot{q} \\
\dot{\theta} \\
\dot{\beta} \\
\dot{p} \\
\dot{r} \\
\dot{\phi}
\end{array}\right]=\left[\begin{array}{cccccccc}
-0.283 & -23.096 & 0 & -0.171 & 0 & 0 & 0 & 0 \\
0 & -3.991 & 0.916 & 0 & 0 & 0 & 0 & 0 \\
0 & -35.922 & -6.539 & 0 & 0 & 0 & 0 & 0 \\
0 & 0 & 1 & 0 & 0 & 0 & 0 & 0 \\
0 & 0 & 0 & 0 & 0.525 & 0.052 & -0.999 & 0 \\
0 & 0 & 0 & 0 & -107.780 & -12.482 & 3.241 & 0 \\
0 & 0 & 0 & 0 & 33.705 & -0.488 & -2.553 & 0 \\
0 & 0 & 0 & 0 & 0 & 1 & 0 & 0
\end{array}\right]\left[\begin{array}{l}
V \\
\alpha \\
q \\
\theta \\
\beta \\
p \\
r \\
\phi
\end{array}\right] } \\
+\left[\begin{array}{ccccccc}
-10.084 & -10.084 & 0 & 0 & 0 & 0 \\
0.338 & 0.338 & \mathbf{0 . 1 5 0} & \mathbf{0 . 1 5 0} & 0 & 0 \\
-33.710 & -33.710 & \mathbf{- 2 . 7 2 0} & \mathbf{- 2 . 7 2 0} & 0 & 0 \\
0 & 0 & 0 & 0 & 0 & 0 \\
\mathbf{- 0 . 3 3 0} & \mathbf{0 . 3 3 0} & -0.240 & 0.240 & -0.248 & -0.248 \\
\mathbf{5 4 . 8 8 0} & \mathbf{- 5 4 . 8 8 0} & 85.186 & -85.186 & 12.776 & 12.776 \\
\mathbf{0 . 5 2 5} & \mathbf{- 0 . 5 2 5} & 0.733 & -0.733 & -14.585 & -14.585 \\
0 & 0 & 0 & 0 & 0 & 0
\end{array}\right]\left[\begin{array}{l}
i_{H_{L}} \\
i_{H_{R}} \\
\delta_{a_{L}} \\
\delta_{a_{R}} \\
\delta_{r_{L}} \\
\delta_{r_{R}}
\end{array}\right]
\end{aligned}
$$

\subsubsection{WVU YF-22 Nominal Stability and Control Derivative Identification Results}

Once the nominal state space model was identified for use in the flight control law design, the next step was to identify stability and control derivatives for application within a flight simulator to test the flight control laws. For the nominal stability and control derivative identification, the nominal state space model was converted to a baseline estimate of the stability and control derivatives using the aircraft geometric and inertial properties. The inertial properties of the WVU YF-22 were estimated using a bifilar torsional pendulum setup and measured experimentally. Table 19 shows the estimated moments of inertia from the experimental setup.

\begin{tabular}{|c|c|}
\hline $\begin{array}{c}\text { Moment of } \\
\text { Inertia }\end{array}$ & $\begin{array}{c}\text { Estimated Value } \\
\left(\mathrm{kg} \mathrm{m}^{2}\right)\end{array}$ \\
\hline$I_{x x}$ & 1.61 \\
\hline$I_{y y}$ & 7.51 \\
\hline $\boldsymbol{I}_{z z}$ & 7.19 \\
\hline
\end{tabular}

Table 19: Estimated Moments of Inertia for WVU YF-22 
Although the moments of inertia were able to be estimated experimentally, it is difficult to accurately estimate $J_{x z}$ using the bifilar torsional pendulum. When estimating $I_{x x}, I_{y y}$, and $I_{z z}$ experimentally, rotation about the corresponding aircraft body axis is easily performed; however, when estimating the product of inertia the measurement is more difficult and does not provide accurate results. Because of these difficulties in experimental estimation, $J_{x z}$ was determined through the optimization process. As the stability and control derivatives were optimized around flight data sets, $J_{x z}$ was also optimized for improved accuracy. Because of the importance of accurate estimation of the inertial properties of the aircraft and the physical difficulties associated with obtaining $J_{x z}$ experimentally, including it in the optimization procedure allowed for the opportunity for a better estimate.

Once the stability and control derivatives were estimated through the state space conversion, they served as a baseline estimate for optimization. Using sections of measured flight data (selected based on wind conditions and maneuvers performed), the output error optimization method was implemented. Control surface deflections as measured in flight were passed through the estimated model. The model output was compared to measured data, with priority going to angle of attack, sideslip, pitch rate, roll rate, and yaw rate. The error between these states was output and implemented into a cost function accounting for the weighting of the states based on priority in optimization. Figure 41 shows the general layout of the optimization scheme for the stability and control derivatives. With each iteration, the stability and control derivatives were updated to minimize the error between the states. 


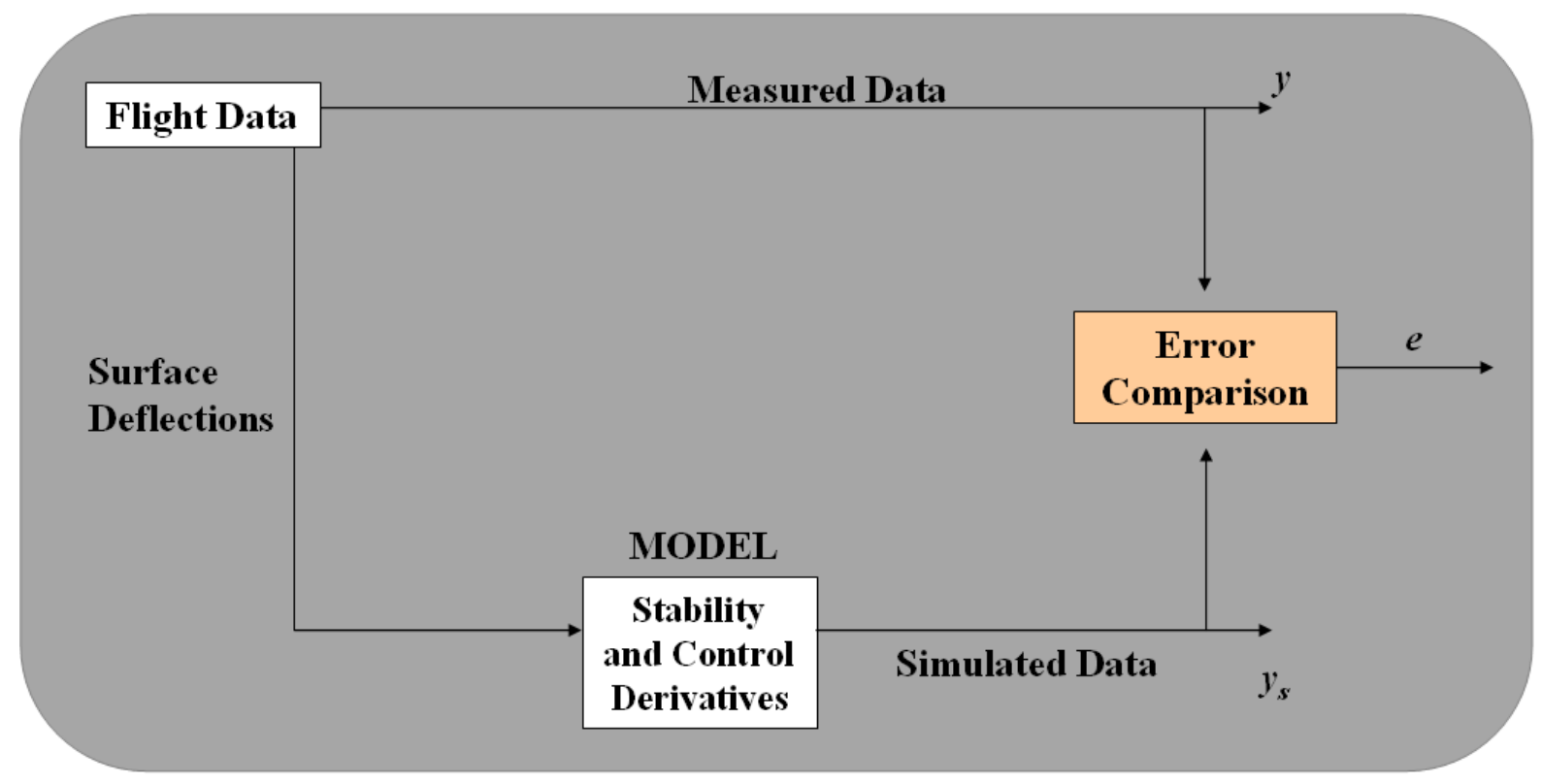

Figure 41: Output Error Optimization of Stability and Control Derivatives

The Matlab ${ }^{\circledR}$ function "costfcn" was developed with the purpose of simulating nonlinear dynamics, using the control deflections from the entire identification data set as inputs to the nonlinear aircraft model, and calculating the value of a cost function based on the RMS of the difference between the "actual" outputs (i.e. the measured output values from the identification data set) and the "simulated" outputs (i.e. the outputs from the nonlinear aircraft model). Therefore, the main input argument of "costfcn" is a vector containing a set of values for the aerodynamic derivatives, along with the product of inertia $J_{x z}$, and the output argument is a single nonnegative scalar number expressing the fitness of that particular set of aerodynamic derivatives and $J_{x z}$.

The "fmincon" function - featuring a constrained optimization of a multivariable function using a Sequential Quadratic Programming technique ${ }^{77}$ - was then used to iteratively minimize the cost function implemented within "costfcn". Essentially the "fmincon" function iteratively calls upon "costfcn" with different inputs, until the set of aerodynamic derivatives - along with the product of inertia $J_{x z}$ - provides the best fit with the flight data. The starting point for this minimization process was the initial set of aerodynamics derivatives. The total cost function was a weighted sum of two cost function terms. The first was a scaled, time-based term given by the RMS of the error between the actual flight data and predicted outputs as shown by Equation 5.7; the second term was a scaled, frequency-based term given by the RMS of the power spectral 
density of the error between the real and predicted outputs as shown by Equation 5.8; finally, the total cost was calculated as a weighted sum of the two cost functions, as shown by Equation 5.9.

$$
J_{T}=N_{T} \sum \sqrt{\overline{(e)^{2}}}
$$

where $N_{T}$ is a scaling factor and $e$ is the error between the actual and predicted outputs.

$$
J_{F}=N_{F} \sum \sqrt{\overline{(E)^{2}}}
$$

where $N_{F}$ is a scaling factor and $E$ is the power spectral density of the error between the actual and predicted outputs.

$$
J=N_{C_{1}} J_{T}+N_{C_{2}} J_{F}
$$

where $N_{C 1}$ and $N_{C 2}$ are scaling factors of 0.1 and 0.9 , respectively.

During the optimization procedure, it became evident that this mathematical process was not yielding results that made physical sense. Estimates for drag coefficients were being produced as negative values, lift coefficients were finalizing at values over 200 and, based on physical knowledge and engineering judgment, were unacceptable. Although it is desirable to have a model that optimizes and represents the aircraft behavior in flight, it is preferred to have a mathematical model of the aircraft that is physically plausible. Different sets of flight data were attempted, but the results were similar. In some cases, optimization was not even a possibility with the simulations diverging. It became apparent that certain bounds would have to be place on some or all of the 30 numbers that were being optimized to hold them at "reasonable" values and directing the optimization procedure. Figures 42 and 43 show samples from the optimization results. Although the pitch rate was optimizing, it was off by $20 \mathrm{deg} / \mathrm{sec}$ during stabilator doublet maneuvers and the drag coefficients produced were negative values. The roll rate response was not optimizing, rather just localizing around the mean value to reduce the cost function. 


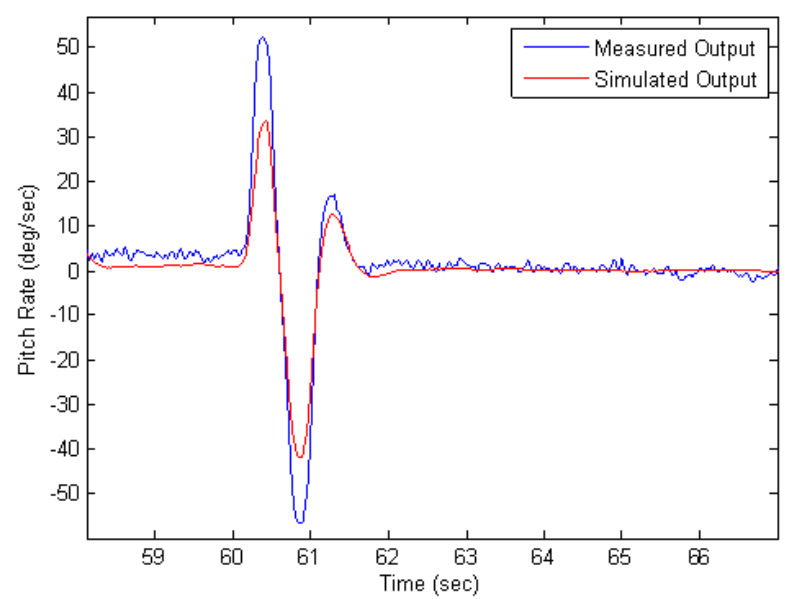

Figure 42: Optimization of YF-22 Pitch Rate

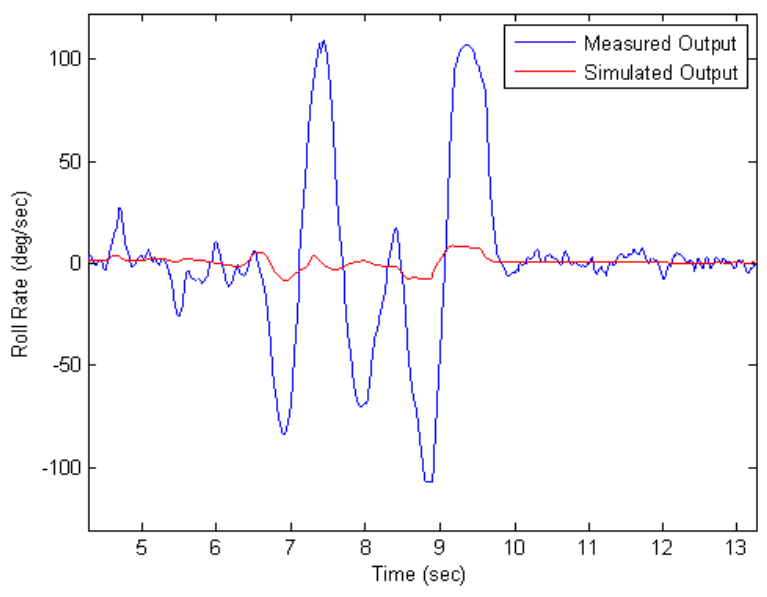

Figure 43: Optimization of YF-22 Roll Rate

It was determined from these trials that pure mathematical optimization would not suffice for the stability and control derivative identification. A means of applying upper and lower bounds to the optimization process on some of the individual coefficients would be necessary, and it was preferred that they were not pure guesses. The next section describes how USAF DATCOM was applied to the WVU YF-22 to estimate some of the stability and control derivatives, and how it was implemented into the optimization process to improve the results.

\subsubsection{WVU YF-22 Application of DATCOM}

DATCOM was utilized for the estimation of several important stability and control derivatives for the WVU YF-22. Since DATCOM was initially developed for large-scale, conventional aircraft, several assumptions were made throughout the estimation process with DATCOM as applied to the WVU YF-22. For optimization purposes, it was evident that several derivatives were "spiking" and did not make physical sense, i.e. negative drag terms were being produced as the final optimized values from the output error method described in section 5.1.2. Of these parameters, several could be estimated using DATCOM methods, so these values were rated in terms of significance and estimated using DATCOM. For the complete description of the calculation of selected longitudinal and lateral-directional stability and control derivatives, please see Appendix A.

Using the measured geometric parameters for the wing, fuselage, and empennage, eleven derivatives were estimated. The estimated values are shown in Table 20. The derivatives assigned the highest priority included the lift and drag coefficients and the sideslip terms. 
Table 20: DATCOM Estimated Values for Selected Stability and Control Derivatives for the WVU YF-22

\begin{tabular}{|c|c|}
\hline Derivative & $\begin{array}{c}\text { DATCOM } \\
\text { Estimated } \\
\text { Value }\end{array}$ \\
\hline$c_{D_{0}}$ & 0.008 \\
\hline$c_{L_{\alpha}}$ & 3.867 \\
\hline$c_{L_{i H}}$ & 0.324 \\
\hline$c_{m_{\alpha}}$ & -0.452 \\
\hline$c_{m_{q}}$ & -0.458 \\
\hline$c_{m_{i H}}$ & -0.408 \\
\hline$c_{l_{\beta}}$ & -0.041 \\
\hline$c_{l_{p}}$ & -0.223 \\
\hline$c_{n_{\beta}}$ & 0.071 \\
\hline$c_{n_{r}}$ & -0.130 \\
\hline$c_{n_{\delta r}}$ & -0.051 \\
\hline
\end{tabular}

From these estimated values and using engineering judgment, upper and lower bounds were implemented into the stability and control optimization process. In addition to adding upper and lower bounds to the estimated parameters, physical bounds were also incorporated on the drag terms to prevent them from optimizing to negative numbers. Varying ranges of constraints were established for the optimization scenarios. It was found that when holding constraints too tightly around these estimated parameters, i.e. 50\% above and below the estimated DATCOM values, the optimization did not produce satisfactory results. Specifically, certain channels did not optimize around the segments of flight data for angle of attack and angular rates. Figures 44 and 45 show sample optimization results whenever 50\% DATCOM constraints were implemented on the selected derivatives. Notice that although the pitch rate optimized well, the roll rate was unable to reach the peaks observed in flight. 


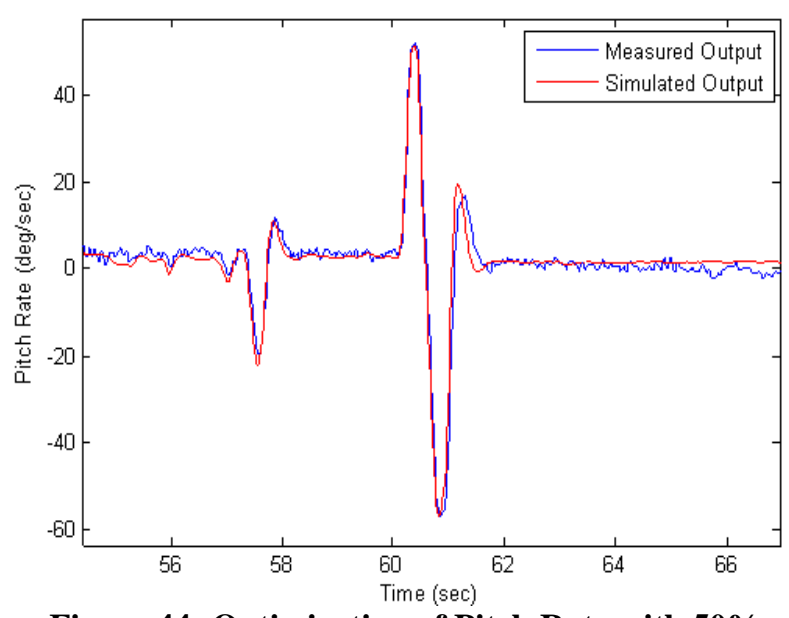

Figure 44: Optimization of Pitch Rate with $50 \%$ DATCOM Constraints

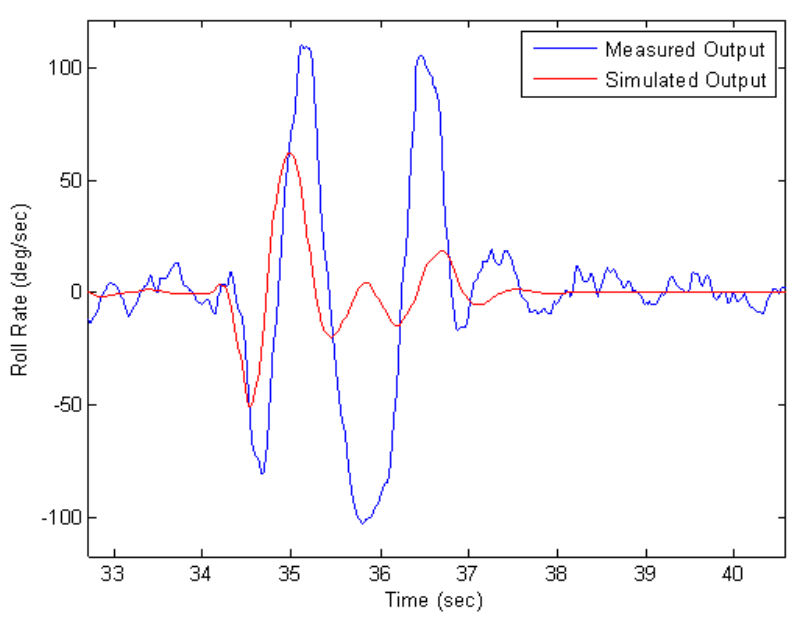

Figure 45: Optimization of Roll Rate with $\mathbf{5 0 \%}$ DATCOM Constraints

Because the DATCOM estimates required geometric estimation, extrapolation of the wind tunnel data on the charts to estimate for the low Mach number, and the general fighter jet configuration of this semi-scale aircraft, the values could have a large error-propagation that is difficult to track or estimate. Due to these uncertainties, these estimated values were used "loosely", that is without $50 \%$ constraining values. Table 21 summarizes the PID and DATCOM estimated values, the error between the estimates, and the lower and upper bounds applied during optimization.

Table 21: PID and DATCOM Estimate Comparison for WVU YF-22

\begin{tabular}{|c|c|c|c|c|c|c|}
\hline Derivative & $\begin{array}{c}\text { PID Estimated } \\
\text { Values }\end{array}$ & $\begin{array}{c}\text { DATCOM } \\
\text { Estimated } \\
\text { Values }\end{array}$ & \% Error & $\begin{array}{c}\text { Applied } \\
\text { Lower } \\
\text { Bounds }\end{array}$ & $\begin{array}{c}\text { Applied } \\
\text { Upper } \\
\text { Bounds }\end{array}$ & $\begin{array}{c}\text { Appropriate } \\
\text { Sign } \\
\text { Convention }\end{array}$ \\
\hline$c_{D_{0}}$ & 0.009 & 0.009 & 2.222 & 0.001 & 0.013 & positive \\
\hline$c_{D_{\alpha}}$ & 0.600 & - & - & 0.000 & - & positive \\
\hline$c_{D_{q}}$ & 0.000 & 0.000 & - & 0.000 & - & positive \\
\hline$c_{D_{i H}}$ & 0.011 & - & - & 0.000 & - & positive \\
\hline$c_{L_{0}}$ & 0.001 & - & - & - & - & either \\
\hline$c_{L_{\alpha}}$ & 3.815 & 3.867 & 1.355 & 1.000 & 5.801 & positive \\
\hline$c_{L_{q}}$ & 0.455 & - & - & - & - & positive \\
\hline$c_{L_{i H}}$ & 0.260 & 0.324 & 24.807 & 0.050 & 0.800 & positive \\
\hline$c_{m_{0}}$ & 0.017 & - & - & - & - & either \\
\hline$c_{m_{\alpha}}$ & -0.313 & -0.452 & 44.409 & -2.000 & 0.000 & either \\
\hline$c_{m_{q}}$ & -0.476 & -0.458 & 3.738 & - & - & negative \\
\hline
\end{tabular}




\begin{tabular}{|c|c|c|c|c|c|c|}
\hline$c_{m_{i H}}$ & -0.280 & -0.408 & 45.610 & -0.800 & 0.000 & negative \\
\hline$c_{Y_{0}}$ & -0.010 & - & - & -0.010 & 0.010 & 0 \\
\hline$c_{Y_{\beta}}$ & -0.339 & -0.127 & - & - & - & negative \\
\hline$c_{Y_{p}}$ & -0.041 & - & - & - & - & negative \\
\hline$c_{Y_{r}}$ & -0.840 & - & - & - & - & positive \\
\hline$c_{Y_{\delta a}}$ & -0.100 & 0.000 & - & - & - & 0 \\
\hline$c_{Y_{\delta r}}$ & -0.011 & - & - & - & - & positive \\
\hline$c_{l_{0}}$ & -0.001 & - & - & -0.010 & 0.010 & 0 \\
\hline$c_{l_{\beta}}$ & -0.077 & -0.041 & 47.348 & -0.090 & -0.010 & negative \\
\hline$c_{l_{p}}$ & -0.350 & -0.223 & 36.268 & -1.000 & -0.100 & negative \\
\hline$c_{l_{r}}$ & 0.017 & - & - & - & - & positive \\
\hline$c_{l_{\delta a}}$ & -0.101 & 0.032 & - & - & - & positive \\
\hline$c_{l_{\delta r}}$ & 0.012 & - & - & - & - & positive \\
\hline$c_{n_{0}}$ & 0.001 & - & - & -0.010 & 0.010 & 0 \\
\hline$c_{n_{\beta}}$ & 0.047 & 0.071 & 50.533 & 0.010 & 0.107 & positive \\
\hline$c_{n_{p}}$ & -0.054 & - & - & - & - & either \\
\hline$c_{n_{r}}$ & -0.139 & -0.130 & 6.273 & -0.500 & -0.050 & negative \\
\hline$c_{n_{\delta a}}$ & -0.016 & - & - & - & - & negative \\
\hline$c_{n_{\delta r}}$ & -0.036 & -0.051 & 42.061 & -0.150 & -0.010 & negative \\
\hline
\end{tabular}

Figures 46 and 47 show the optimization results from a nominal stabilator doublet on the angle pitch rate and angle of attack responses, respectively. 


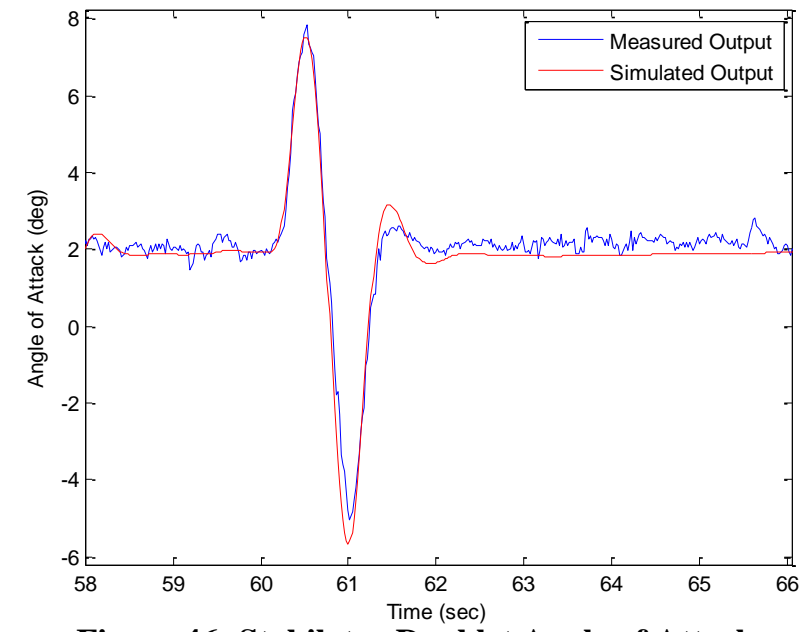

Figure 46: Stabilator Doublet Angle of Attack

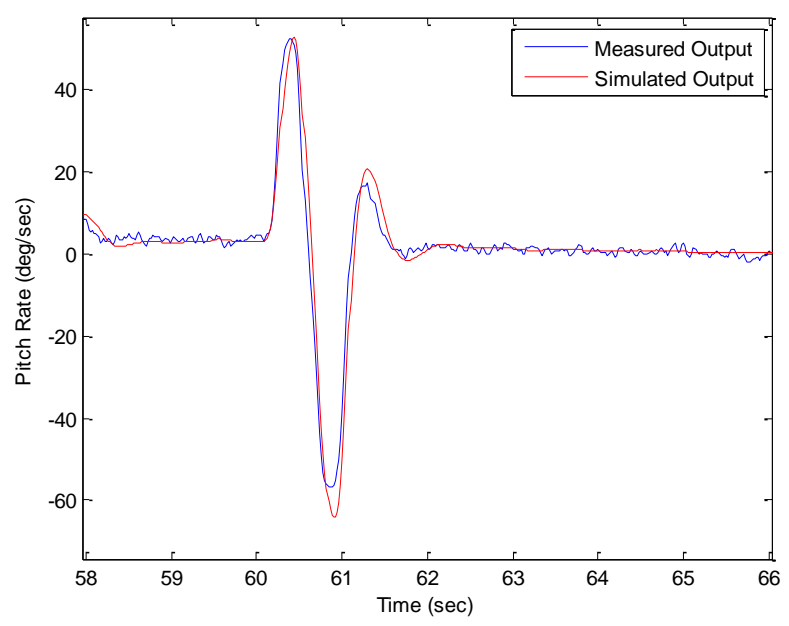

Figure 47: Stabilator Doublet Pitch Rate Response

Figures 48 and 49 show the optimization results from a nominal rudder/aileron doublet combination on the roll rate and yaw rate responses, respectively.

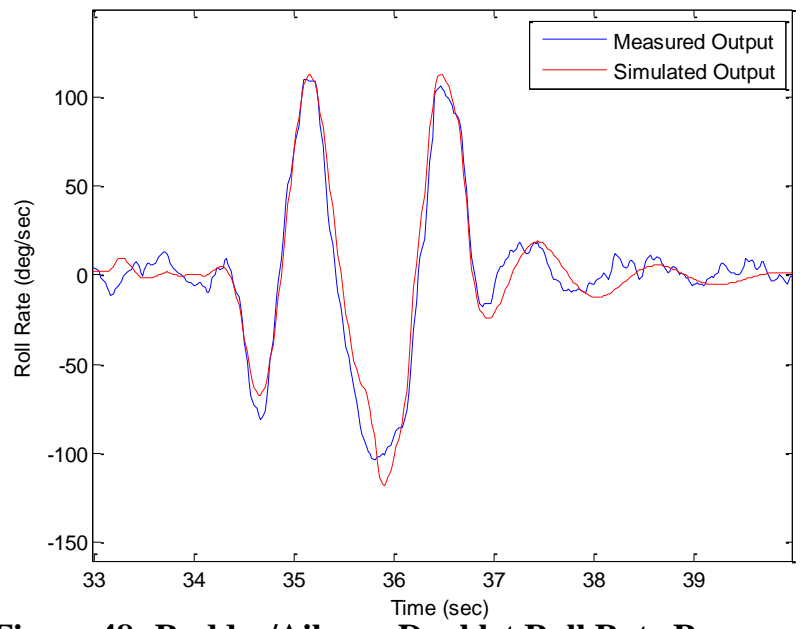

Figure 48: Rudder/Aileron Doublet Roll Rate Response

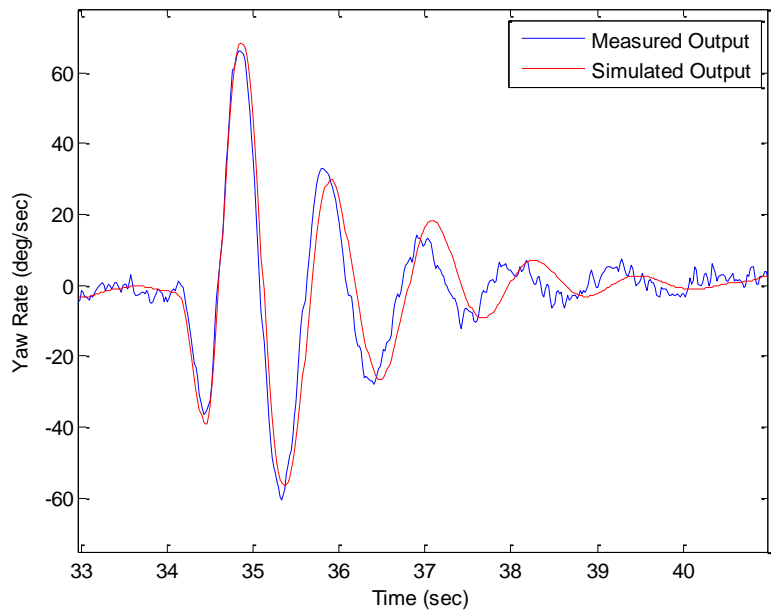

Figure 49: Rudder/Aileron Doublet Yaw Rate Response

A lift-to-drag ratio analysis was conducted from the estimated stability and control derivatives representing lift and drag on the WVU YF-22. Table 22 summarizes the estimated lift and drag coefficients and the lift-to-drag ratio as predicted through PID.

Table 22: Lift-to-Drag Ratio Analysis from PID Values for WVU YF-22

\begin{tabular}{|c|c|c|c|c|c|c|c|c|c|c|}
\hline Aircraft & $c_{D_{0}}$ & $c_{D_{\alpha}}$ & $c_{D_{i H}}$ & $c_{L_{0}}$ & $c_{L_{\alpha}}$ & $c_{L_{q}}$ & $c_{L_{i H}}$ & $C_{D}$ & $C_{L}$ & $L / D$ \\
\hline WVU YF-22 & 0.009 & 0.600 & 0.011 & 0.001 & 3.815 & 0.455 & 0.260 & 0.620 & 4.531 & $\mathbf{7 . 3 0}$ \\
\hline
\end{tabular}

With the successful optimization using DATCOM, the next step was to identify the CoDICE stability and control derivatives for a complete simulation scheme to test flight control laws with individual control surface movements. 


\subsubsection{WVU YF-22 CoDICE Stability and Control Derivative Identification Results}

Once the nominal stability and control derivatives were optimized, the control derivatives representative of the cross-coupling of the longitudinal and lateral-directional dynamics could be identified. Because no damage to control surfaces was being modeled, the optimized nominal stability and control derivatives will remain unchanged. The contributions of the optimized nominal control derivatives were halved to represent the effects of the individual control surface on the flight dynamics. The success of optimizing the cross-coupled terms depended on the accuracy of the nominal model in representing the aircraft since that portion of the CoDICE model was held constant in this process. Figure 50 shows the CoDICE stability and control derivative layout, with the derivatives highlighted in the yellow boxes as those cross-coupled terms that require optimization.

$$
\begin{aligned}
& c_{D}(\xi, \delta)=c_{D 0}+c_{D \alpha} \alpha+c_{D q} \frac{\bar{c}}{2 V} q+c_{D_{i H_{L}}} i_{H_{L}}+c_{D_{i H_{R}}} i_{H_{R}}+c_{D_{\delta A_{L}}} \boldsymbol{\delta}_{A_{L}}+c_{D_{\delta A_{R}}} \boldsymbol{\delta}_{A_{R}}+c_{D_{\delta R_{L}}} \boldsymbol{\delta}_{R_{\Sigma}}+c_{D_{\delta R_{R}}} \boldsymbol{\delta}_{R_{R}} \\
& c_{L}(\xi, \delta)=c_{L 0}+c_{L \alpha} \alpha+c_{L q} \frac{\bar{c}}{2 V} q+c_{L_{H_{I}}} i_{H_{L}}+c_{L_{i H_{R}}} i_{H_{R}}+c_{L_{\delta A_{L}}} \delta_{A_{\Sigma}}+c_{L_{\delta A R}} \delta_{A_{R}}+c_{L_{\delta R_{L}}} \delta_{R_{L}}+c_{L_{\delta R_{R}}} \delta_{R_{R}} \\
& c_{m}(\xi, \delta)=c_{m 0}+c_{m \alpha} \alpha+c_{m q} \frac{\bar{c}}{2 V} q+c_{m_{i_{L}}} i_{H_{L}}+c_{m_{i H_{R}}} i_{H_{R}}+c_{m_{\delta A_{L}}} \boldsymbol{\delta}_{A_{L}}+c_{m_{\delta A R}} \boldsymbol{\delta}_{A_{R}}+c_{m_{\delta R_{L}}} \boldsymbol{\delta}_{R_{L}}+c_{m_{\delta R_{R}}} \boldsymbol{\delta}_{R_{R}} \\
& c_{Y}(\xi, \delta)=c_{Y 0}+c_{Y \beta} \beta+c_{Y p} \frac{b}{2 V} p+c_{I y} \frac{b}{2 V} r+c_{Y i_{H_{L}}} i_{H_{L}}+c_{Y i_{H_{R}}} i_{H_{R}}+c_{Y \delta_{A_{L}}} \delta_{A_{L}}+c_{Y \delta_{A_{R}}} \delta_{A_{R}}+c_{Y \delta_{R_{L}}} \delta_{R_{L}}+c_{Y \delta_{R_{R}}} \delta_{R_{R}} \\
& c_{l}(\xi, \delta)=c_{l 0}+c_{l \beta} \beta+c_{l p} \frac{b}{2 V} p+c_{l r} \frac{b}{2 V} r+c_{l_{H_{L}}} i_{H_{L}}+c_{l i_{H R}} i_{H_{R}}+c_{l \delta_{A L}} \delta_{A_{L}}+c_{l \delta_{A R}} \delta_{A_{R}}+c_{l \delta_{R_{L}}} \delta_{R_{L}}+c_{l \delta_{R R}} \delta_{R_{R}} \\
& c_{n}(\xi, \delta)=c_{n 0}+c_{n \beta} \beta+c_{n p} \frac{b}{2 V} p+c_{n r} \frac{b}{2 V} r+c_{n i_{H_{L}}} i_{H_{L}}+c_{n i_{H_{R}}} i_{H_{R}}+c_{n \delta_{A_{L}}} \delta_{A_{L}}+c_{n \delta_{A_{R}}} \delta_{A_{R}}+c_{n \delta_{R_{L}}} \delta_{R_{L}}+c_{n \delta_{R_{R}}} \delta_{R_{R}}
\end{aligned}
$$

Figure 50: CoDICE Stability and Control Derivative Representation

The output error optimization method was utilized again to estimate the cross-coupled terms shown in Figure 50. The flight data selected for the optimization process included the segments of measured flight were primary control surface failures were induced. The optimization scheme focused solely on optimizing the twelve unknown terms. Initial attempts were made to optimize these terms without constraints, but values again exceed what would be considered physically possible. For example, the aileron contributions to angle of attack and pitch rate were exceeding those contributions from the stabilators. 
The results of the initial CoDICE model optimization were reviewed during the doublet maneuvers with control surface failures. Figures 51 and 52 show the optimization results from a stabilator doublet with left stabilator failure on the roll rate and from an aileron doublet with left aileron failure on the pitch rate, respectively.

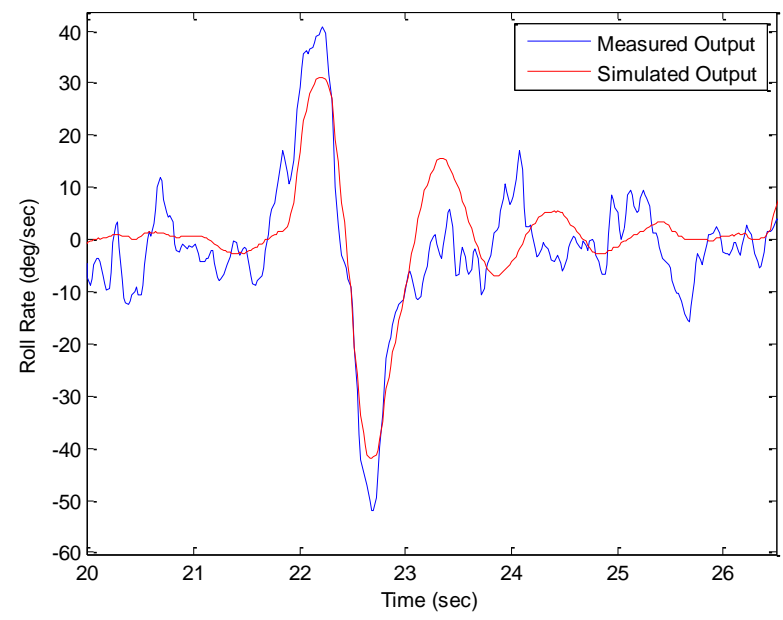

Figure 51: Individual Stabilator Doublet Roll Rate Response

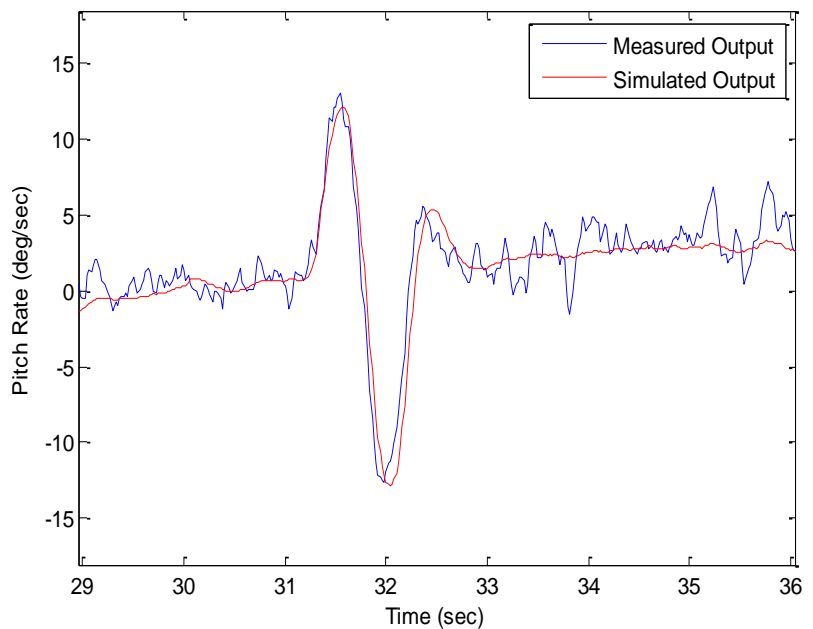

Figure 52: Individual Aileron Doublet Pitch Rate Response

It was observed that the roll rate response under stabilator failure conditions did not optimize sufficiently. Because of these insufficient results, upper and lower bounds were again implemented during the optimization process, with the bounds based on the nominal derivative contributions on the cross-coupling terms. Table 23 shows the optimization results from the trial without constraints and from the final model were upper and lower bounds were implemented.

Table 23: Optimization Results for the Unknown CoDICE Control Derivatives

\begin{tabular}{|c|c|c|c|c|}
\hline & $\begin{array}{c}\text { No } \\
\text { Constraints } \\
\text { (PID) }\end{array}$ & $\begin{array}{c}\text { Implementing } \\
\text { Bounds (PID) }\end{array}$ & $\begin{array}{c}\text { Lower } \\
\text { Bounds }\end{array}$ & $\begin{array}{c}\text { Upper } \\
\text { Bounds }\end{array}$ \\
\hline$c_{D_{\delta a_{L}}}$ & 0.010 & 0.001 & 0 & 0.005 \\
\hline$c_{D_{\delta a_{R}}}$ & 0.010 & 0.001 & 0 & 0.005 \\
\hline$c_{L_{\delta a_{L}}}$ & 0.102 & 0.012 & 0 & 0.05 \\
\hline$c_{L_{\delta a_{R}}}$ & 0.102 & 0.012 & 0 & 0.05 \\
\hline$c_{m_{\delta a_{L}}}$ & -0.055 & -0.062 & -0.1 & 0 \\
\hline$c_{m_{\delta a_{R}}}$ & -0.055 & -0.062 & -0.1 & 0 \\
\hline$c_{Y_{i H_{L}}}$ & 0.055 & 0.001 & - & - \\
\hline$c_{Y_{i H_{R}}}$ & -0.055 & -0.001 & - & - \\
\hline
\end{tabular}




\begin{tabular}{|l|l|l|l|l|}
$c_{l_{i H_{L}}}$ & 0.033 & 0.045 & 0.05 & 0 \\
\hline$c_{l_{i H_{R}}}$ & -0.033 & -0.045 & -0.05 & 0 \\
\hline$c_{n_{i H_{L}}}$ & 0.019 & 0.001 & 0.12 & 0 \\
\hline$c_{n_{i H_{R}}}$ & -0.019 & -0.001 & -0.12 & 0 \\
\hline
\end{tabular}

Again, the cost function during the optimization was the same as implemented during the nominal model methods described in Section 5.1.2, with the greatest focus being on the roll rate and pitch rate responses from the cross-coupled terms. Figures 53 and 54 show the final optimization results for the roll rate and pitch rate responses under control surface failure conditions.

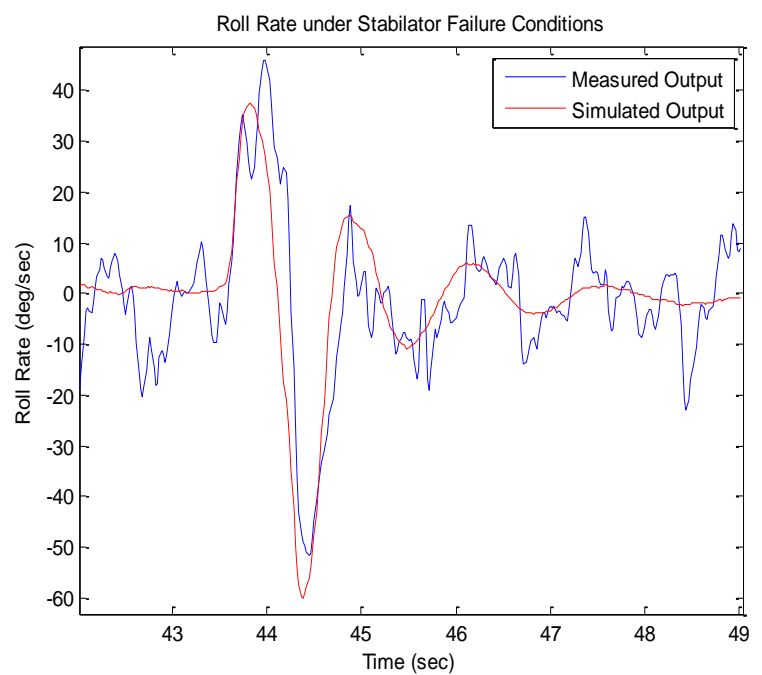

Figure 53: Individual Stabilator Doublet Roll Rate Response

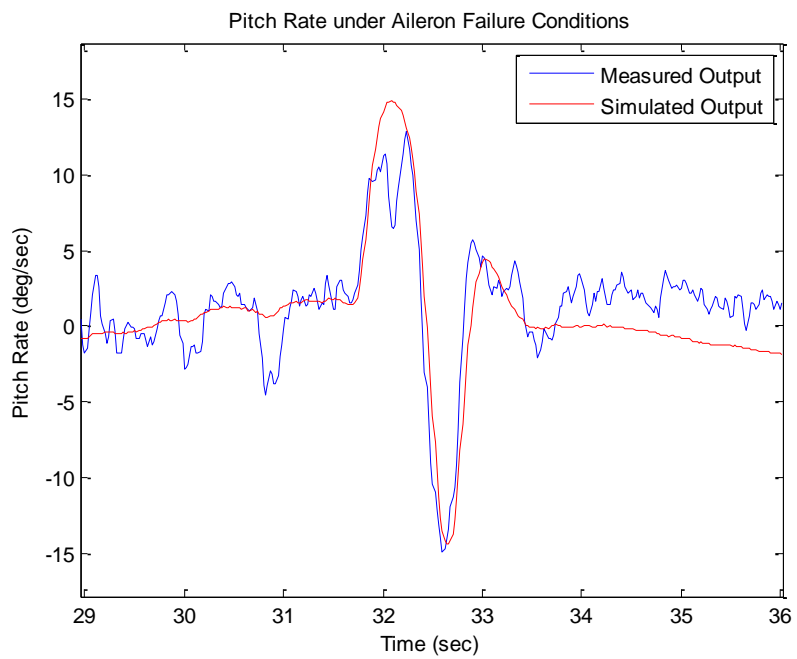

Figure 54: Individual Aileron Doublet Pitch Rate Response

Table 24 shows the complete CoDICE model that was used for simulation of the flight control laws. 
Table 24: Estimated CoDICE Stability and Control Derivatives

\begin{tabular}{|c|c|c|c|}
\hline Term & $\begin{array}{l}\text { Implementing } \\
\text { Bounds (PID) }\end{array}$ & Term & $\begin{array}{l}\text { Implementing } \\
\text { Bounds (PID) }\end{array}$ \\
\hline$c_{D_{0}}$ & 0.009 & $c_{Y_{p}}$ & -0.041 \\
\hline$c_{D_{\alpha}}$ & 0.601 & $c_{Y_{r}}$ & -0.840 \\
\hline$c_{D_{q}}$ & 0 & $c_{Y_{i H_{L}}}$ & 0.001 \\
\hline$c_{D_{i H_{L}}}$ & 0.005 & $c_{Y_{i H_{R}}}$ & -0.001 \\
\hline$c_{D_{i H_{R}}}$ & 0.005 & $c_{Y_{\delta a_{L}}}$ & 0.050 \\
\hline$c_{D_{\delta a_{L}}}$ & 0.002 & $c_{Y_{\delta a_{R}}}$ & -0.050 \\
\hline$c_{D_{\delta a_{R}}}$ & 0.002 & $c_{Y_{\delta_{r_{L}}}}$ & -0.005 \\
\hline$c_{D_{\delta_{r_{L}}}}$ & 0 & $c_{Y_{\delta_{r_{R}}}}$ & -0.005 \\
\hline$c_{D_{\delta_{r_{R}}}}$ & 0 & $c_{l_{0}}$ & -0.001 \\
\hline$c_{L_{0}}$ & 0.001 & $c_{l_{\beta}}$ & -0.077 \\
\hline$c_{L_{\alpha}}$ & 3.815 & $c_{l_{p}}$ & -0.350 \\
\hline$c_{L_{q}}$ & 0.455 & $c_{l_{r}}$ & 0.017 \\
\hline$c_{L_{i H_{L}}}$ & 0.130 & $c_{l_{i H_{L}}}$ & 0.045 \\
\hline$c_{L_{i H_{R}}}$ & 0.130 & $c_{l_{i H_{R}}}$ & -0.045 \\
\hline$c_{L_{\delta a_{L}}}$ & 0.012 & $c_{l_{\delta a_{L}}}$ & 0.051 \\
\hline$c_{L_{\delta a_{R}}}$ & 0.012 & $c_{l_{\delta a_{R}}}$ & -0.051 \\
\hline$c_{L_{\delta_{r_{L}}}}$ & 0 & $c_{l_{\delta_{r_{L}}}}$ & 0.006 \\
\hline$c_{L_{\delta_{r_{R}}}}$ & 0 & $c_{l_{\delta_{r_{R}}}}$ & 0.006 \\
\hline$c_{m_{0}}$ & 0.017 & $c_{n_{0}}$ & 0.001 \\
\hline$c_{m_{\alpha}}$ & -0.313 & $c_{n_{\beta}}$ & 0.047 \\
\hline$c_{m_{q}}$ & -0.476 & $c_{n_{p}}$ & -0.054 \\
\hline$c_{m_{i H_{L}}}$ & -0.140 & $c_{n_{r}}$ & -0.139 \\
\hline$c_{m_{i H_{R}}}$ & -0.140 & $c_{n_{i_{L}}}$ & 0.001 \\
\hline$c_{m_{\delta a_{L}}}$ & -0.062 & $c_{n_{i H_{R}}}$ & -0.001 \\
\hline$c_{m_{\delta a_{R}}}$ & -0.062 & $c_{n_{\delta a_{L}}}$ & 0.008 \\
\hline$c_{m_{\delta_{r_{L}}}}$ & 0 & $c_{n_{\delta a_{R}}}$ & -0.008 \\
\hline$c_{m_{\delta_{r_{R}}}}$ & 0 & $c_{n_{\delta_{r_{L}}}}$ & -0.018 \\
\hline$c_{Y_{0}}$ & -0.010 & $c_{n_{\delta_{r_{R}}}}$ & -0.018 \\
\hline$c_{Y_{\beta}}$ & -0.339 & $I_{x z}\left(k g m^{2}\right)$ & -0.003 \\
\hline
\end{tabular}




\subsubsection{WVU YF-22 Simulation Studies}

After deriving the CoDICE mathematical model, simulation studies were conducted to validate its performance. During periods of nominal aircraft flight, i.e. no primary control surface failures, the cross-coupling terms cancel according to sign convention of the model. During periods of control surface failures, the cross-coupling terms become significant and are necessary to mimic the flight behaviors observed in actual flight. The first set of simulation studies included maneuvers where the aircraft was flying under nominal conditions. For these simulations, maneuvers from measured flight data were passed through the CoDICE model and the output states were compared to those observed in actual flight. Figures 55 and 56 show the angle of attack and pitch rate responses due to a nominal stabilator doublet, respectively. Figures 57 and 58 show the sideslip and yaw rate responses due to a rudder/aileron doublet combination maneuver, respectively.

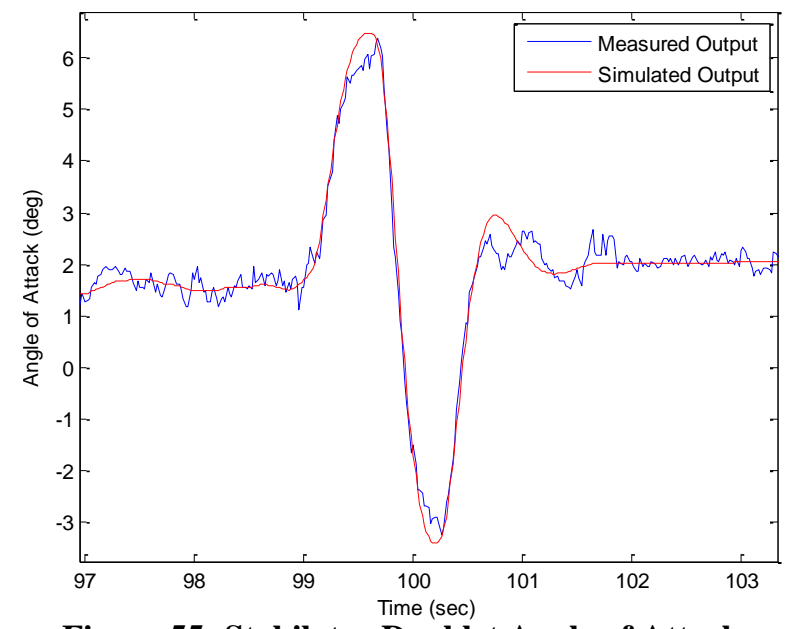

Figure 55: Stabilator Doublet Angle of Attack

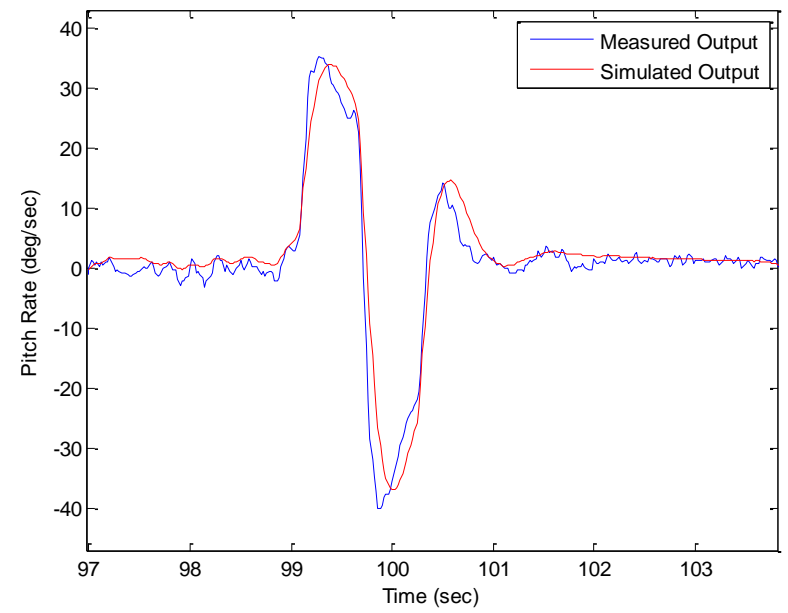

Figure 56: Stabilator Doublet Pitch Rate Response 


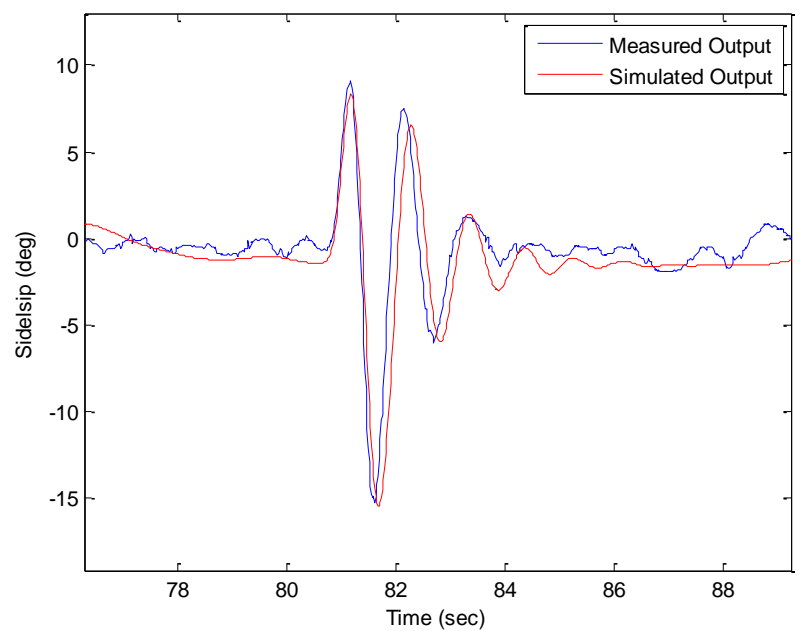

Figure 57: Rudder/Aileron Doublet Sideslip

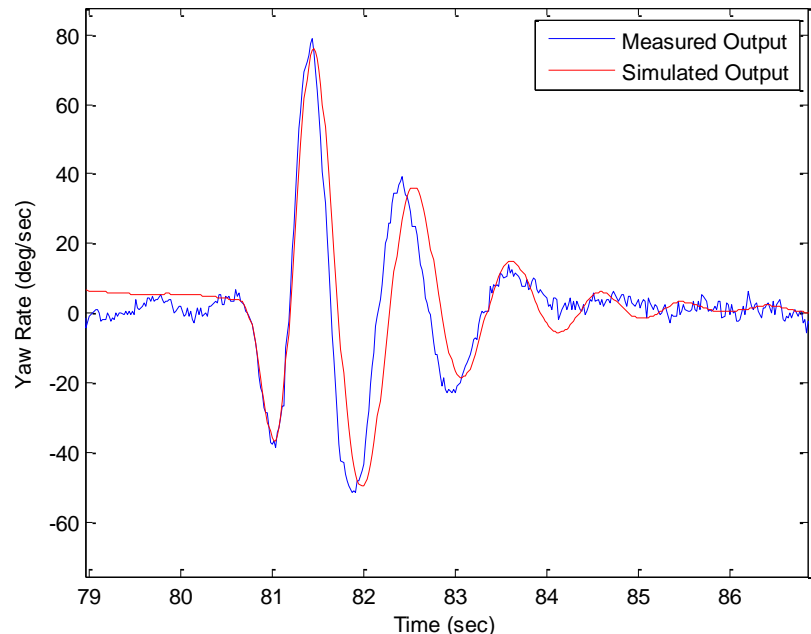

Figure 58: Rudder/Aileron Doublet Yaw Rate

Figures 59 and 60 show roll rate responses to two different rudder/aileron doublet combination maneuvers.

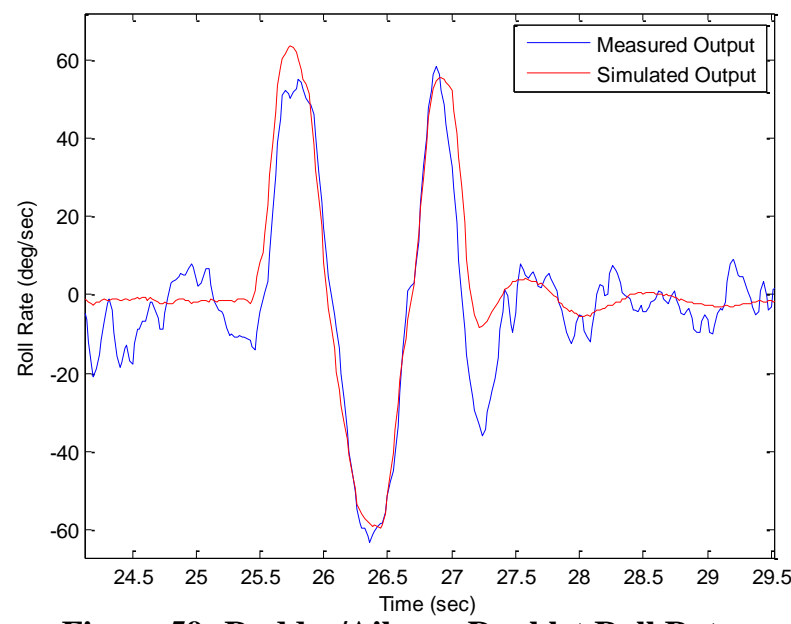

Figure 59: Rudder/Aileron Doublet Roll Rate

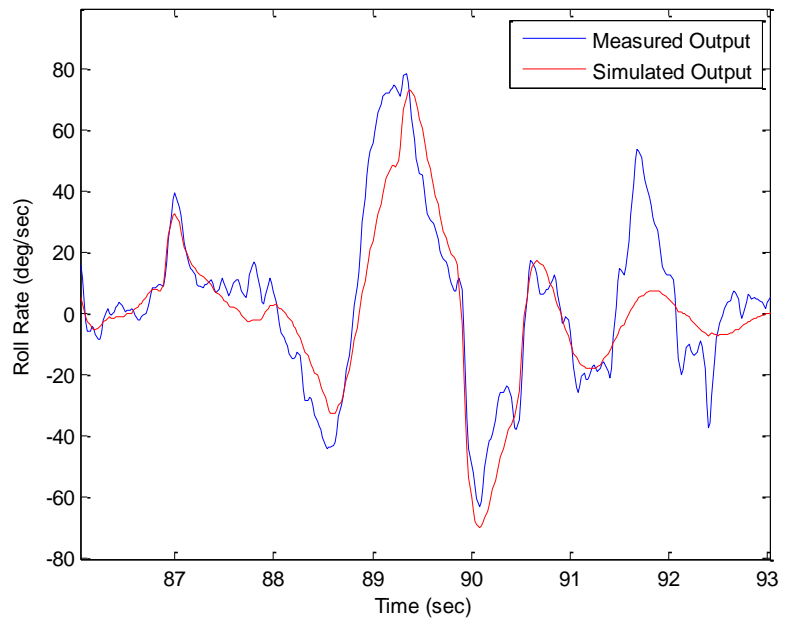

Figure 60: Rudder/Aileron Doublet Roll Rate

Figures 61 and 62 show the sideslip and yaw rate responses for another rudder/aileron doublet maneuver. 


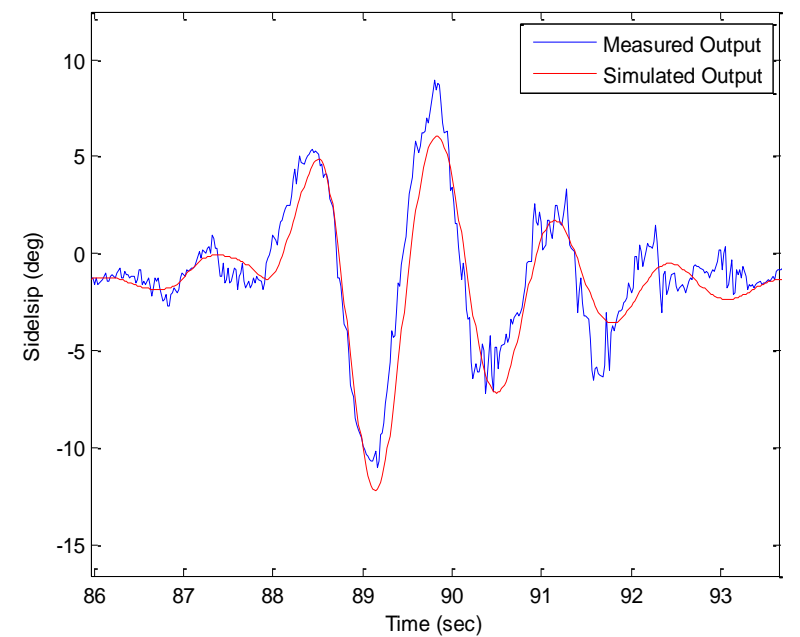

Figure 61: Rudder/Aileron Doublet Sideslip

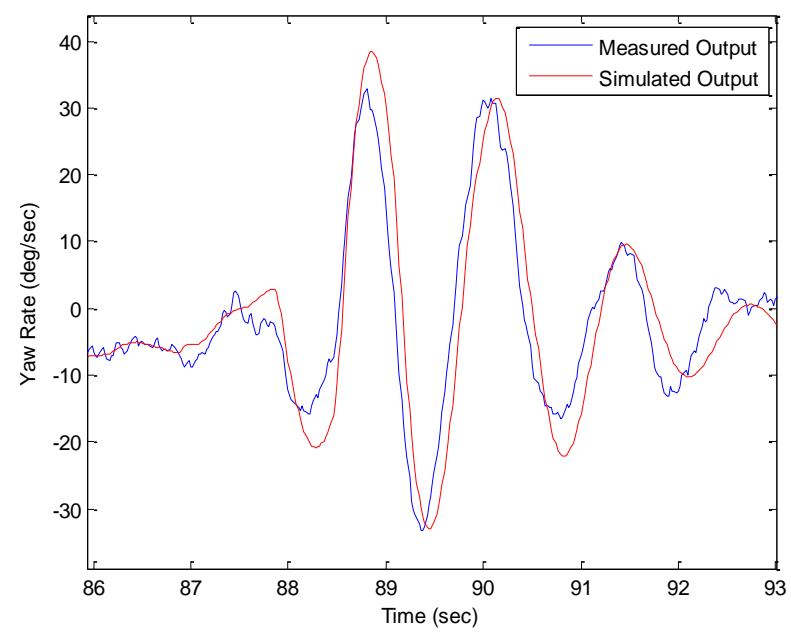

Figure 62: Rudder/Aileron Doublet Yaw Rate

In each of the nominal maneuvers shown above, there was a strong correlation between the measured and simulated output. Next, a simulation study was conducted to determine the performance of the CoDICE model under primary control surface failures. Figures 63 and 64 show simulation results comparing the simulated model output to measured flight data during periods of these control surface failures. Specifically, Figure 63 shows the pitch and roll rate response comparison during an individual stabilator maneuver performed by the OBES in actual flight. Figure 64 shows the pitch and roll rate response comparison during an individual stabilator maneuver performed by the pilot in actual flight.
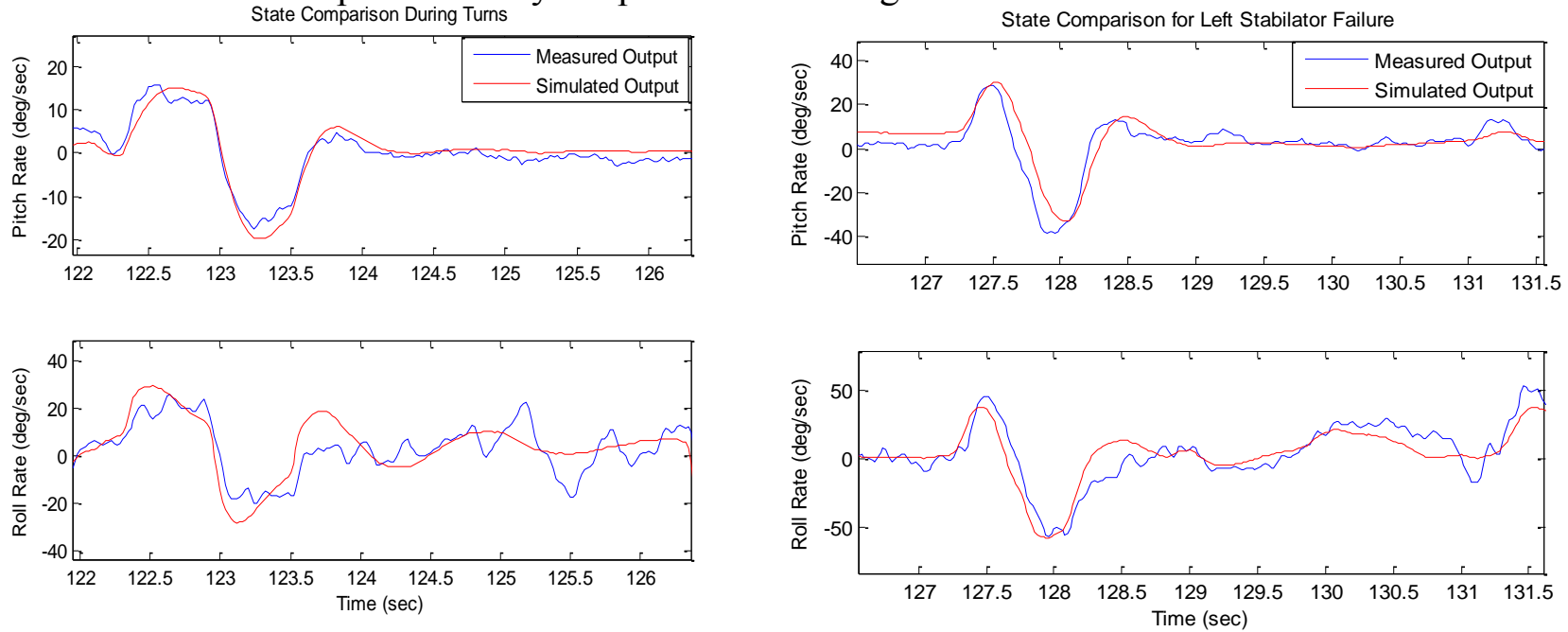

Figure 63: Pitch and Roll Rate Response to Single OBES Stabilator Doublet

Figure 64: Pitch and Roll Rate Response to Single Manual Stabilator Doublet

Figures 65 and 66 show simulation results comparing the simulated model output to measured flight data during periods of aileron failures. Specifically, Figure 65 shows the pitch and roll rate 
response comparison during an individual aileron maneuver performed by the OBES in actual flight. Figure 66 shows the pitch and roll rate response comparison during an individual aileron maneuver performed by the pilot in actual flight.
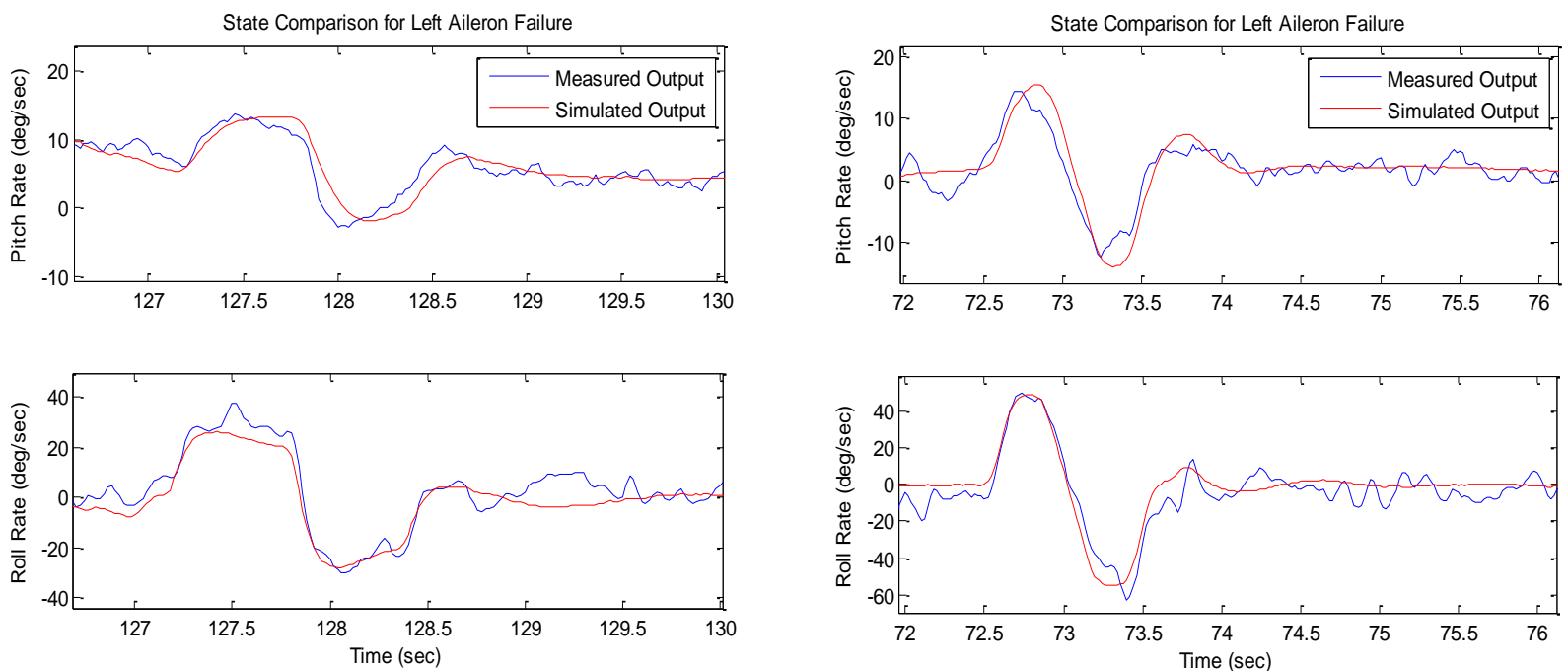

Figure 65: Pitch and Roll Rate Response to Single OBES Aileron Doublet

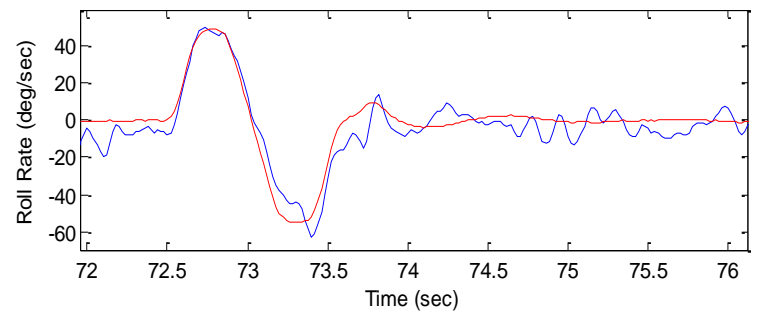

Figure 66: Pitch and Roll Rate Response to Single Manual Aileron Doublet

Figure 67 shows the simulated angle of attack response during an individual aileron maneuver. Figure 68 showcases a longer segment of flight, specifically during a period of a highly banked turn where the aircraft enters into nonlinear flight conditions. During this period of flight, the aircraft was flying under healthy conditions, and the linear model represented what was observed in flight data under nonlinear flight conditions.

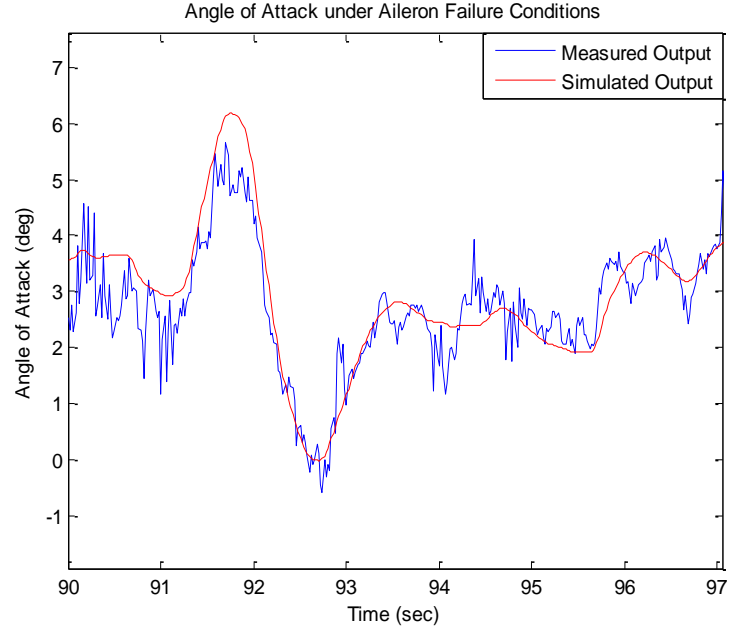

Figure 67: Angle of Attack Response to Single Manual Aileron Doublet
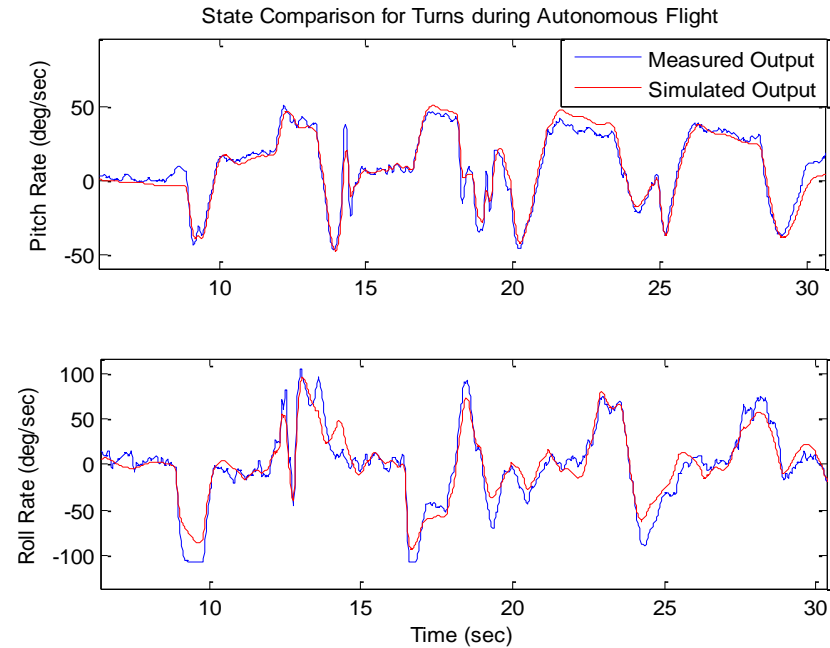

Figure 68: Pitch and Roll Rate Response during Highly Banked Turns 
The maneuvers shown above represent a sample of a larger group of maneuvers that were tested during nominal and control surface failure conditions. The CoDICE model responses were compared to actual flight data and an error comparison was conducted Table 25 shows the average error and average standard deviation of the error between the simulated and actual aircraft responses. These values were obtained after averaging the values over a set of 9 maneuvers for each case. The largest error is observed in the roll rate response; however, in many of the maneuvers the roll rate reached a magnitude of $100 \mathrm{deg} / \mathrm{sec}$ so the error is less than $10 \%$ of the total response. A sample of data consisting of an entire flight with nominal maneuvers was also passed through the model and compared to actual flight data.

Table 25: Average Error Analysis between Measured and Simulated Output

\begin{tabular}{|c|c|c|c|c|c|c|c|c|}
\hline & \multicolumn{2}{|c|}{$\begin{array}{c}\text { Angle of Attack } \\
\text { (deg) }\end{array}$} & \multicolumn{2}{c|}{$\begin{array}{c}\text { Pitch Rate } \\
\text { (deg/sec) }\end{array}$} & \multicolumn{2}{c|}{$\begin{array}{c}\text { Roll Rate } \\
\text { (deg/sec) }\end{array}$} & \multicolumn{2}{c|}{$\begin{array}{c}\text { Yaw Rate } \\
\text { (deg/sec) }\end{array}$} \\
\hline Maneuver & $\begin{array}{c}\text { Mean } \\
\text { Error }\end{array}$ & $\begin{array}{c}\text { Standard } \\
\text { Deviation }\end{array}$ & $\begin{array}{c}\text { Mean } \\
\text { Error }\end{array}$ & $\begin{array}{c}\text { Standard } \\
\text { Deviation }\end{array}$ & $\begin{array}{c}\text { Mean } \\
\text { Error }\end{array}$ & $\begin{array}{c}\text { Standard } \\
\text { Deviation }\end{array}$ & $\begin{array}{c}\text { Mean } \\
\text { Error }\end{array}$ & $\begin{array}{c}\text { Standard } \\
\text { Deviation }\end{array}$ \\
\hline $\begin{array}{c}\text { Nominal } \\
\text { Stabilator }\end{array}$ & 0.23 & 0.55 & 0.15 & 4.07 & - & - & - & - \\
\hline $\begin{array}{c}\text { Nominal } \\
\text { Rudder/Aileron }\end{array}$ & - & - & - & - & 2.80 & 8.95 & 0.44 & 6.72 \\
\hline $\begin{array}{c}\text { Stabilator with } \\
\text { Failure }\end{array}$ & 0.07 & 0.40 & 0.27 & 3.44 & 1.62 & 7.82 & - & - \\
\hline $\begin{array}{c}\text { Aileron with } \\
\text { Failure }\end{array}$ & 0.21 & 0.23 & 1.82 & 2.32 & 2.04 & 7.06 & - & - \\
\hline $\begin{array}{c}\text { Nominal Entire } \\
\text { Flight (cruise) }\end{array}$ & 0.02 & 0.36 & 0.72 & 2.16 & 0.43 & 7.03 & 0.53 & 4.29 \\
\hline
\end{tabular}

For further evaluation, the CoDICE stability and control derivatives were implemented into a Simulink ${ }^{\circledR}$ environment developed to simulate the aircraft following a Virtual Leader path similar to those used in flight tests. Figure 69 shows simulation results for the model (red) following the VL path (blue) with an OBES performing maneuvers during the straight legs of the flight. On the left side of the figure, a stabilator doublet is performed with a left stabilator failure; therefore, the simulated track of the aircraft climbs slightly but also slightly rolls out of the path as it was observed in actual flight. On the right side of Figure 69, a nominal aileron doublet was performed so the simulator rolled the aircraft out of the track significantly, as observed in flight. 


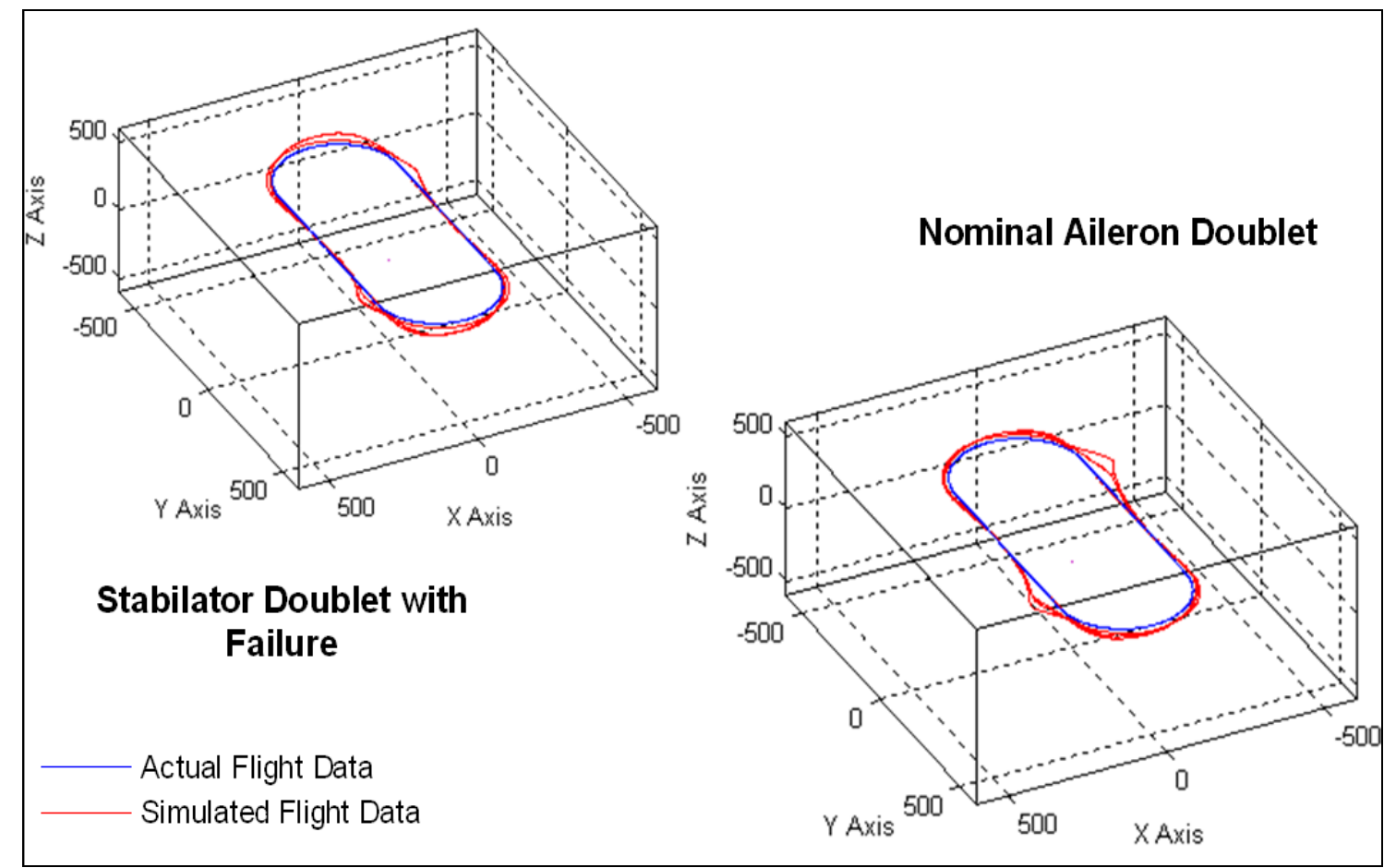

Figure 69: Simulation Results for Virtual Leader Tracking with OBES Maneuvers

Based on the simulation studies and error analysis, it was determined that the identified model of the WVU YF-22 performs as expected. The longitudinal and lateral-directional dynamic responses were accurately represented under both nominal and primary control surface failure conditions through the CoDICE model. It is expected that this model will meet expectations for use within a fault-tolerant flight control system for application toward handling actuator failures.

\subsubsection{WVU YF-22 Confidence Interval Analysis}

While obtaining estimates for the WVU YF-22 state space parameters from flight data, it was important to also predict the level of confidence that could be placed on the estimates. That is, what is the predicted range of error for the parameter estimates and what are the $95 \%$ confidence intervals for those identified values. Once the state space model was identified and optimized, a confidence interval analysis was implemented.

A confidence interval analysis was conducted on the state space parameters through the optimization process. This provided a predicted percent error calculation and a $95 \%$ confidence interval for the identified parameters in the nominal state space model. The parameters were separately optimized for the longitudinal and lateral-directional dynamics, thus the confidence intervals were also separately evaluated. 
The identified parameters for the longitudinal state space model include values in the state and input matrices that affect angle of attack and pitch rate. The parameters are identified based on their positions in the state or input matrix as follows:

$$
\left[\begin{array}{c}
\dot{\alpha} \\
\dot{q}
\end{array}\right]=\left[\begin{array}{ll}
A(1,1) & A(1,2) \\
A(2,1) & A(2,2)
\end{array}\right]\left[\begin{array}{l}
\alpha \\
q
\end{array}\right]+\left[\begin{array}{c}
B(1) \\
B(2)
\end{array}\right]\left[i_{H}\right]
$$

Table 26 shows the results of the confidence interval analysis for the longitudinal state space parameters. The segment of flight data used for optimization was also used to determine the $95 \%$ confidence intervals and error analysis. The standard error is equivalent to the standard deviation of the sample mean based on the population mean (i.e. the measured flight data used for optimization). The $95 \%$ confidence intervals for each estimated parameter are based on the sample flight data used for optimization. The confidence intervals provide an estimated value range for the flight data and where the parameter estimate should fall.

Table 26: Longitudinal YF-22 State Space Estimates, Error Analyses, and $95 \%$ Confidence Intervals

\begin{tabular}{|c|c|c|c|c|c|}
\hline Parameter & Estimate & $\begin{array}{c}\text { Standard } \\
\text { Error }\end{array}$ & \% Error & $\begin{array}{c}\text { Confidence } \\
\text { Interval } \\
\text { (Lower) }\end{array}$ & $\begin{array}{c}\text { Confidence } \\
\text { Interval } \\
\text { (Upper) }\end{array}$ \\
\hline $\mathbf{A}(\mathbf{1 , 1})$ & -3.991 & 0.034 & 0.90 & -4.059 & -3.923 \\
\hline $\mathbf{A}(\mathbf{1 , 2})$ & 0.916 & 0.123 & 1.30 & 0.891 & 0.941 \\
\hline $\mathbf{A}(\mathbf{2}, \mathbf{1})$ & -35.922 & 0.277 & 0.80 & -36.475 & -35.369 \\
\hline $\mathbf{A}(\mathbf{2}, \mathbf{2})$ & -6.539 & 0.114 & 1.70 & -6.768 & -6.311 \\
\hline $\mathbf{B}(\mathbf{1})$ & 0.675 & 0.075 & 11.10 & 0.525 & 0.824 \\
\hline $\mathbf{B}(\mathbf{2})$ & -67.420 & 0.702 & 1.00 & -68.823 & -66.016 \\
\hline
\end{tabular}

The identified parameters for the lateral-directional state space model include values in the state and input matrices that affect sideslip, roll rate, and yaw rate. The parameters are identified based on their positions in the state or input matrix as follows:

$$
\left[\begin{array}{c}
\dot{\beta} \\
\dot{p} \\
\dot{r}
\end{array}\right]=\left[\begin{array}{lll}
A(1,1) & A(1,2) & A(1,3) \\
A(2,1) & A(2,2) & A(2,3) \\
A(3,1) & A(3,2) & A(3,3)
\end{array}\right]\left[\begin{array}{l}
\beta \\
p \\
r
\end{array}\right]+\left[\begin{array}{ll}
B(1,1) & B(1,2) \\
B(2,1) & B(2,2) \\
B(3,1) & B(3,2)
\end{array}\right]\left[\begin{array}{l}
\delta_{a} \\
\delta_{r}
\end{array}\right]
$$

Table 27 shows the results of the confidence interval analysis for the lateral-directional state space parameters. The segment of flight data used for optimization was also used to determine the $95 \%$ confidence intervals and error analysis. 
Table 27: Lateral-Directional YF-22 State Space Estimates, Error Analyses, and 95\% Confidence Intervals

\begin{tabular}{|c|c|c|c|c|c|}
\hline Parameter & Estimate & $\begin{array}{l}\text { Standard } \\
\text { Error }\end{array}$ & $\%$ Error & $\begin{array}{l}\text { Confidence } \\
\text { Interval } \\
\text { (Lower) }\end{array}$ & $\begin{array}{l}\text { Confidence } \\
\text { Interval } \\
\text { (Upper) }\end{array}$ \\
\hline$A(1,1)$ & 0.525 & 0.007 & 1.30 & 0.511 & 0.539 \\
\hline$A(2,1)$ & -107.780 & 1.640 & 1.50 & -111.059 & -104.501 \\
\hline$A(2,2)$ & -12.482 & 0.022 & 1.70 & -12.917 & -12.047 \\
\hline$A(2,3)$ & 3.241 & 0.170 & 5.30 & 2.900 & 3.582 \\
\hline$A(3,1)$ & 33.705 & 0.084 & 0.30 & 33.536 & 33.874 \\
\hline$A(3,3)$ & -2.553 & 0.016 & 0.60 & -2.586 & -2.520 \\
\hline$B(1,2)$ & -0.497 & 0.002 & 4.90 & -0.545 & -0.448 \\
\hline$B(2,1)$ & \begin{tabular}{|l|}
-170.372 \\
\end{tabular} & 2.795 & 1.60 & -175.962 & -164.782 \\
\hline$B(2,2)$ & 25.552 & 1.182 & 4.60 & 23.187 & 27.916 \\
\hline $\mathrm{B}(3,1)$ & -1.466 & 0.109 & 7.40 & -1.684 & -1.247 \\
\hline$B(3,2)$ & -29.170 & 0.175 & 0.60 & -29.520 & -28.819 \\
\hline
\end{tabular}

The standard error and percent error estimates for the identified longitudinal and lateraldirectional state space parameters were low. One longitudinal input matrix estimate exceeded $10 \%$ error, and the $95 \%$ confidence intervals indicated a small varying range for the upper and lower bounds. These results indicate that based on the measured flight data used for optimization, the estimated parameters will accurately represent what was observed in flight. Following this analysis, this identified state space model was sufficient to be utilized for flight control law design.

\subsection{WVU PACT Results}

Following the successful identification of the WVU YF-22 parameters, a similar method was employed to identify a nominal state space model and stability and control derivatives for the WVU PACT aircraft. The following sections detail the results of the parameter identification study for the PACT aircraft, and further show the need for DATCOM estimates in the stability and control derivative optimization process.

\subsubsection{WVU PACT Linear State Space Model Identification Results}

Parameter identification flight tests were conducted during the 2010 flight season with the WVU PACT to estimate a nominal mathematical model of the aircraft. Specific maneuvers were performed with the control surfaces to excite the short period, Dutch roll, and roll responses of the aircraft. Doublet maneuvers were performed on the elevators to excite the short period, and rudder and aileron doublets were performed independently to excite the Dutch roll and roll modes, respectively. These maneuvers were performed by a pilot and Figures 70 and 71 show 
samples of flight data used for parameter identification with a elevator doublet and rudder doublet, respectively. These maneuvers were performed by the pilot and represent the type of data segmenting that was used in the identification process of the nominal state space model. Each maneuver performed during flight tests were segmented to show the input maneuver as well as the system dynamic response.

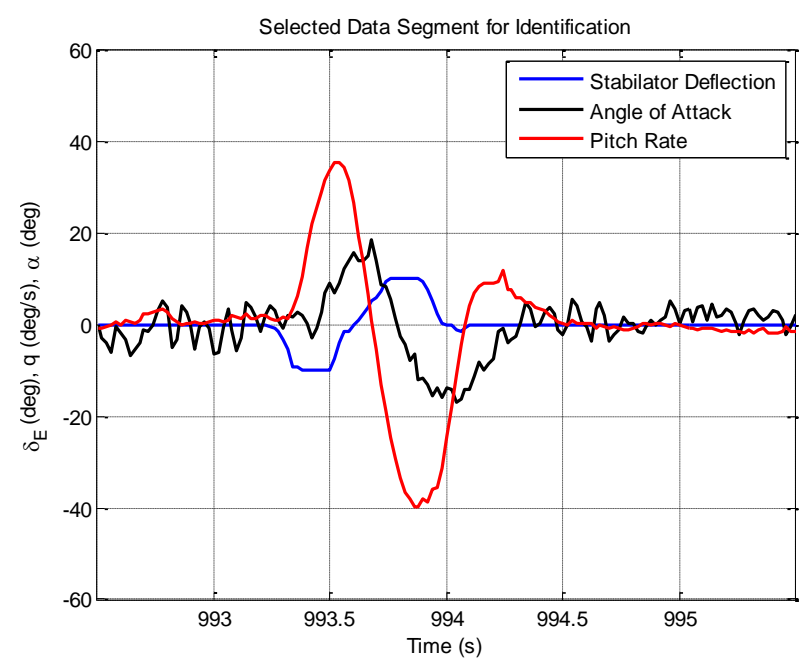

Figure 70: Flight Data Segment used for Longitudinal Linear Model Identification

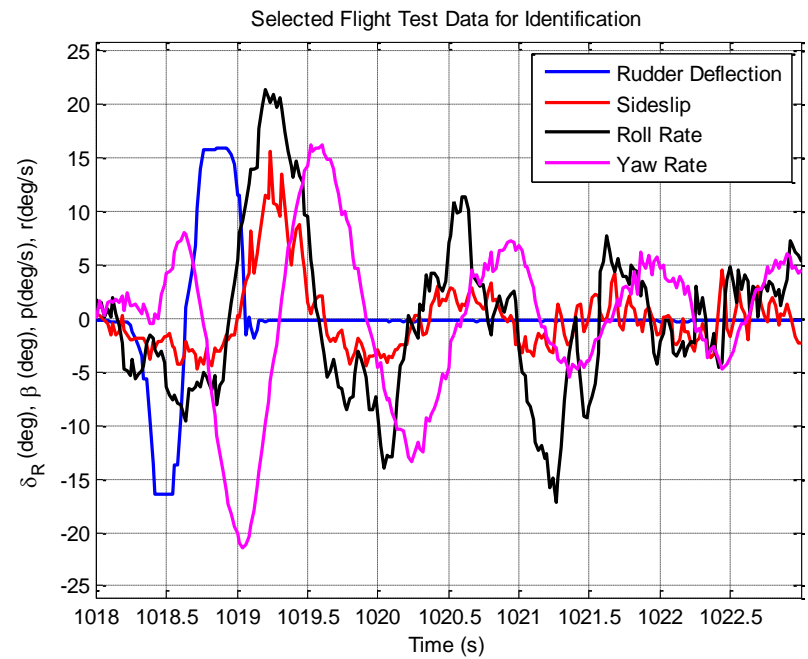

Figure 71: Flight Data Segment used for LateralDirectional Model Identification

Table 28 summarizes the maneuvers that were used for off-line PID analysis for the short period mode, and Table 30 summarizes the maneuvers used for off-line analysis of the Dutch roll and roll modes. Tables 29 and 31 highlight the average, median, and standard deviation of the damping ratios and natural frequencies of the selected modes. The values in red represent values that were not used in the estimation of the roll mode or Dutch roll mode since it was not the primary excited state.

A total of 18 maneuvers were performed for the short period analysis. Table 28 highlights the flight data, maneuver performed, time segment from that flight, eigenvalues of the short period mode, the short period damping ratio, and the short period natural frequency. Table 29 shows the statistical analysis of the short period mode, specifically the average, median, and standard deviation of the damping ratio and natural frequency. Selected outliers were removed and the analysis was repeated for these statistical measures and reported in Table 29.

Table 30 shows the 23 maneuvers performed to identify the Dutch roll and roll modes. Table 30 also shows the flight data, maneuver performed, time segment from that flight, eigenvalues of the Dutch roll and roll modes, and the damping ratio and natural frequency of the Dutch roll. 
Table 31 shows the statistical analysis of the Dutch roll and roll modes. The average, median, and standard deviation of the roll time constant and the Dutch roll damping ratio and natural frequency were evaluated. Again, major outliers were removed from the data and these statistical results were also reported in Table 31.

Table 28: Elevator Maneuvers and Resulting Short Period Properties for WVU PACT

\begin{tabular}{|c|c|c|c|c|c|c|}
\hline \multicolumn{7}{|c|}{ Longitudinal PID Results } \\
\hline Flight Date & Maneuver & Time Segment & $\begin{array}{c}\text { Eigenvalues (Short } \\
\text { Period) }\end{array}$ & Damping Ratio & $\begin{array}{c}\text { Natural } \\
\text { Frequency (rps) } \\
\end{array}$ & Number \\
\hline $11 / 22 / 2010$ & Elevator & $780-784$ & $-5.42 \pm 5.75 \mathrm{i}$ & 0.69 & 7.90 & 1 \\
\hline $11 / 22 / 2010$ & Elevator & $803-806$ & $-5.58 \pm 5.37 \mathrm{i}$ & 0.72 & 7.74 & 2 \\
\hline $11 / 22 / 2010$ & Elevator & $819-823.5$ & $-5.00 \pm 3.87 \mathrm{i}$ & 0.79 & 6.32 & 3 \\
\hline $11 / 22 / 2010$ & Elevator & $841-844$ & $-6.11 \pm 8.29 \mathrm{i}$ & 0.59 & 10.30 & 4 \\
\hline $11 / 22 / 2010$ & Elevator & $862-865$ & $-6.42 \pm 6.63 \mathrm{i}$ & 0.70 & 9.23 & 5 \\
\hline $11 / 22 / 2010$ & Elevator & $992-996$ & $-5.20 \pm 8.91 \mathrm{i}$ & 0.50 & 10.31 & 6 \\
\hline $11 / 22 / 2010$ & Elevator & $542-545.5$ & $-5.29 \pm 8.25 \mathrm{i}$ & 0.54 & 9.80 & 7 \\
\hline $11 / 22 / 2010$ & Elevator & $560.5-563$ & $-4.46 \pm 6.48 \mathrm{i}$ & 0.57 & 7.87 & 8 \\
\hline $11 / 22 / 2010$ & Elevator & $563-566$ & $-6.82 \pm 9.40 \mathrm{i}$ & 0.59 & 11.61 & 9 \\
\hline $11 / 22 / 2010$ & Elevator & $566-570$ & $-5.82 \pm 7.95 \mathrm{i}$ & 0.59 & 9.85 & 10 \\
\hline $11 / 22 / 2010$ & Elevator & $585.5-588$ & $-4.82 \pm 7.14 i$ & 0.56 & 8.61 & 11 \\
\hline $11 / 22 / 2010$ & Elevator & $588-591$ & $-5.16 \pm 7.47 \mathrm{i}$ & 0.57 & 9.08 & 12 \\
\hline $11 / 22 / 2010$ & Elevator & $721-724$ & $-5.50 \pm 7.44 \mathrm{i}$ & 0.59 & 9.25 & 13 \\
\hline $11 / 22 / 2010$ & Elevator & $724-728$ & $-4.92 \pm 8.01 \mathrm{i}$ & 0.52 & 9.40 & 14 \\
\hline $11 / 22 / 2010$ & Elevator & $795.5-798$ & $-4.96 \pm 8.18 \mathrm{i}$ & 0.52 & 9.57 & 15 \\
\hline $11 / 22 / 2010$ & Elevator & $560-570$ & $-5.46 \pm 8.02 \mathrm{i}$ & 0.56 & 9.70 & 16 \\
\hline $11 / 22 / 2010$ & Elevator & $585-591$ & $-5.03 \pm 7.17 \mathrm{i}$ & 0.57 & 8.76 & 17 \\
\hline $11 / 22 / 2010$ & Elevator & $721-728$ & $-5.31 \pm 7.90 \mathrm{i}$ & 0.56 & 9.52 & 18 \\
\hline
\end{tabular}

Table 29: Short Period Damping Ratio and Natural Frequency Statistical Analysis of PACT

\begin{tabular}{|c|c|c|c|}
\hline & & Damping Ratio & Natural Frequency (rps) \\
\hline & Average & 0.60 & 9.16 \\
\hline & Median & 0.57 & 9.33 \\
\hline & Standard Deviation & 0.08 & 1.19 \\
\cline { 2 - 4 } & Average & 0.58 & 9.29 \\
\cline { 2 - 4 } Removed Outliers & Median & 0.57 & 9.33 \\
\cline { 2 - 4 } & Standard Deviation & 0.04 & 0.39 \\
\hline
\end{tabular}


Table 30: Aileron and Rudder Maneuvers and Resulting Dutch Roll and Roll Properties of WVU PACT

\begin{tabular}{|c|c|c|c|c|c|c|c|}
\hline \multicolumn{8}{|c|}{ Lateral-Directional PID Results } \\
\hline Flight Date & Maneuver & Time Segment & $\begin{array}{l}\text { Eigenvalues } \\
\text { (Dutch Roll) }\end{array}$ & \begin{tabular}{|c|} 
Eigenvalues \\
(Roll)
\end{tabular} & \begin{tabular}{|c} 
Damping \\
Ratio
\end{tabular} & \begin{tabular}{|c|}
$\begin{array}{c}\text { Natural } \\
\text { Frequency } \\
\text { (rps) }\end{array}$ \\
\end{tabular} & Number \\
\hline $11 / 22 / 2010$ & Aileron & $886-890$ & $-0.95 \pm 3.34 \mathrm{i}$ & -14.23 & - & - & 1 \\
\hline $11 / 22 / 2010$ & Aileron & $907-910$ & $-0.07 \pm 5.72 \mathrm{i}$ & -16.92 & - & - & 2 \\
\hline $11 / 22 / 2010$ & Aileron & $914-916.5$ & $-1.80 \pm 2.09 \mathrm{i}$ & -11.64 & - & - & 3 \\
\hline $11 / 22 / 2010$ & Aileron & $1004-1008$ & $-0.72 \pm 3.83 \mathrm{i}$ & -15.32 & - & - & 4 \\
\hline $11 / 22 / 2010$ & Rudder & $928-931.1$ & $-0.73 \pm 5.12 \mathrm{i}$ & -0.89 & 0.14 & 5.17 & 5 \\
\hline $11 / 22 / 2010$ & Rudder & $948-952$ & $-1.215 \pm 5.44 \mathrm{i}$ & -13.53 & 0.22 & 5.57 & 6 \\
\hline $11 / 22 / 2010$ & Rudder & $969-979$ & $-0.90 \pm 5.36 \mathrm{i}$ & -9.29 & 0.17 & 5.43 & 7 \\
\hline $11 / 22 / 2010$ & Rudder & $1018-1022$ & $-0.54 \pm 4.89 \mathrm{i}$ & -5.46 & 0.11 & 4.92 & 8 \\
\hline $11 / 22 / 2010$ & Aileron & $605-607.5$ & $-1.12 \pm 3.82 \mathrm{i}$ & -12.00 & - & - & 9 \\
\hline $11 / 22 / 2010$ & Aileron & $607.5-610.5$ & $-0.79 \pm 5.11 \mathrm{i}$ & -9.79 & - & - & 10 \\
\hline $11 / 22 / 2010$ & Aileron & $626-628.5$ & $-2.14 \pm 2.09 \mathrm{i}$ & -16.31 & - & - & 11 \\
\hline $11 / 22 / 2010$ & Aileron & $628.5-633$ & $-1.10 \pm 4.53 \mathrm{i}$ & -12.02 & - & - & 12 \\
\hline $11 / 22 / 2010$ & Aileron & $746-748.5$ & $-0.21 \pm 1.89 \mathrm{i}$ & -15.75 & - & - & 13 \\
\hline $11 / 22 / 2010$ & Aileron & $748.5-751$ & $-0.73 \pm 4.94 \mathrm{i}$ & -12.69 & - & - & 14 \\
\hline $11 / 22 / 2010$ & Aileron & $752-755$ & $-1.44 \pm 2.9176 \mathrm{i}$ & -10.89 & - & - & 15 \\
\hline $11 / 22 / 2010$ & Rudder & $645-648.5$ & $-0.42 \pm 5.40 \mathrm{i}$ & -5.17 & 0.08 & 5.41 & 16 \\
\hline $11 / 22 / 2010$ & Rudder & $648.5-650.45$ & $-1.19 \pm 6.48 \mathrm{i}$ & -2.84 & 0.18 & 6.59 & 17 \\
\hline $11 / 22 / 2010$ & Rudder & $680.5-683$ & $-0.85 \pm 6.28 \mathrm{i}$ & -16.88 & 0.13 & 6.34 & 18 \\
\hline $11 / 22 / 2010$ & Rudder & $701-704.5$ & $-0.76 \pm 4.75 i$ & -30.08 & 0.16 & 4.81 & 19 \\
\hline $11 / 22 / 2010$ & Rudder & $704.5-707$ & $-0.55 \pm 4.90 \mathrm{i}$ & -21.47 & 0.11 & 4.93 & 20 \\
\hline $11 / 22 / 2010$ & Rudder & $767-770$ & $-1.33 \pm 5.99 \mathrm{i}$ & -1.60 & 0.22 & 6.13 & 21 \\
\hline $11 / 22 / 2010$ & Rudder & $771-773$ & $-1.11 \pm 6.08 \mathrm{i}$ & 0.49 & 0.18 & 6.18 & 22 \\
\hline $11 / 22 / 2010$ & Aileron & $746-755$ & $-0.59 \pm 5.14 \mathrm{i}$ & -15.45 & - & - & 23 \\
\hline
\end{tabular}

Table 31: Roll Time Constant and Dutch Roll Damping Ratio and Natural Frequency Statistical Analysis

\begin{tabular}{|c|c|c|c|c|}
\hline & & $\begin{array}{c}\text { Roll Time } \\
\text { Constant (sec) }\end{array}$ & Damping Ratio & $\begin{array}{c}\text { Natural } \\
\text { Frequency (rps) }\end{array}$ \\
\hline & Average & 13.58 & 0.15 & 5.59 \\
\hline & Median & 13.46 & 0.16 & 5.43 \\
\hline & Standard Deviation & 2.36 & 0.04 & 0.63 \\
\hline \multirow{4}{*}{$\begin{array}{c}\text { Removed } \\
\text { Outliers }\end{array}$} & Average & 13.64 & 0.15 & 5.55 \\
\cline { 2 - 5 } & Mtandard Deviation & 1.74 & 0.16 & 5.43 \\
\hline
\end{tabular}


The resulting continuous-time nominal longitudinal and lateral-directional linear models were identified as follows using the System Identification Toolbox:

$$
\begin{gathered}
{\left[\begin{array}{c}
\dot{\alpha} \\
\dot{q} \\
\dot{\theta}
\end{array}\right]=\left[\begin{array}{ccc}
-4.9479 & 4.9962 & 0 \\
-11.1305 & -6.0498 & 0 \\
0 & 1 & 0
\end{array}\right]\left[\begin{array}{l}
\alpha \\
q \\
\theta
\end{array}\right]+\left[\begin{array}{c}
4.4013 \\
-36.5839 \\
0
\end{array}\right] \delta_{e}} \\
{\left[\begin{array}{c}
\dot{\beta} \\
\dot{p} \\
\dot{r} \\
\dot{\phi}
\end{array}\right]=\left[\begin{array}{cccc}
-2.2840 & 0.2972 & -1.2493 & 0 \\
-58.9501 & -15.0550 & 7.8743 & 0 \\
17.3082 & -2.1257 & -1.3215 & 0 \\
0 & 1 & 0 & 0
\end{array}\right]\left[\begin{array}{l}
\beta \\
p \\
r \\
\phi
\end{array}\right]+\left[\begin{array}{cc}
-2.0829 & -0.5444 \\
104.3050 & 3.4673 \\
-4.9390 & -4.2805 \\
0 & 0
\end{array}\right]\left[\begin{array}{l}
\delta_{a} \\
\delta_{r}
\end{array}\right]}
\end{gathered}
$$

The corresponding eigenvalues along with the damping, natural frequency, and time constant values for the dynamic modes are listed in Table 32.

Table 32: Eigenvalues, Damping, and Natural Frequencies of WVU PACT Aircraft

\begin{tabular}{|c|c|c|c|}
\hline Dynamic Mode & Eigenvalues & Damping & $\begin{array}{c}\text { Natural Frequency } \\
(\mathbf{r a d} / \mathbf{s e c})\end{array}$ \\
\hline Short Period & $-5.50 \pm 7.44 \mathrm{i}$ & 0.59 & 9.25 \\
\hline Dutch Roll & $-2.71 \pm 5.84 \mathrm{i}$ & 0.42 & 6.44 \\
\hline Roll & 13.24 & - & - \\
\hline
\end{tabular}

The state space models represented in Equations 5.12 and 5.13 were utilized as a starting point for the output error optimization scheme. Segments of flight data were selected based on the input maneuver, and several maneuvers were "meshed" together as if they were recorded consecutively. Figures 72 and 73 show flight data segments that were used in the optimization process. These inputs include elevator doublets for the longitudinal optimization and a rudder and aileron doublet for the lateral-directional optimization. Figures 74 and 75 show the optimization results, that is, show the optimized model state output as compared to the measured flight data. 


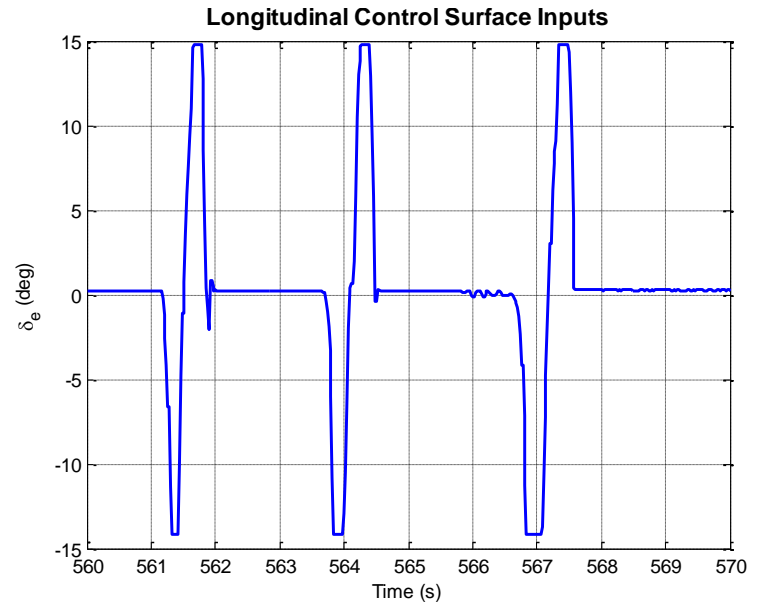

Figure 72: Measured Elevator Deflections used for Optimization
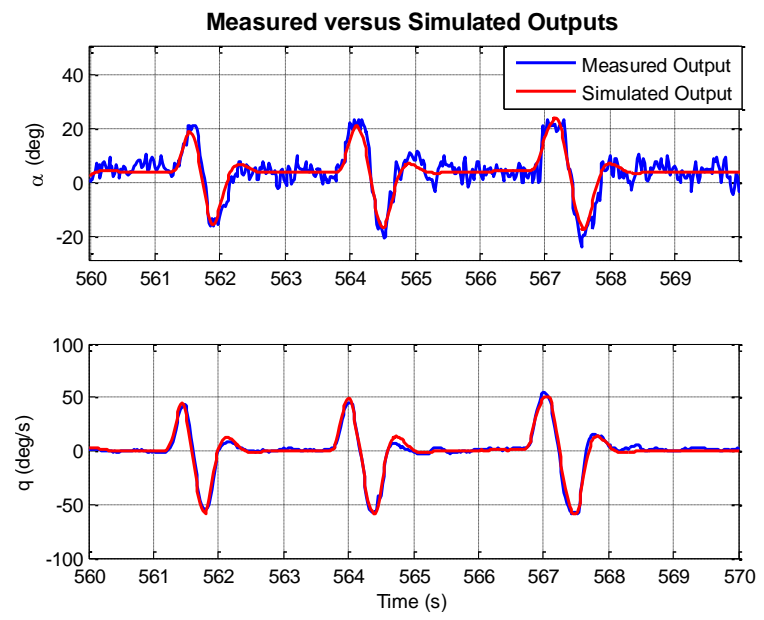

Figure 74: Optimization Results for Angle of Attack and Pitch Rate
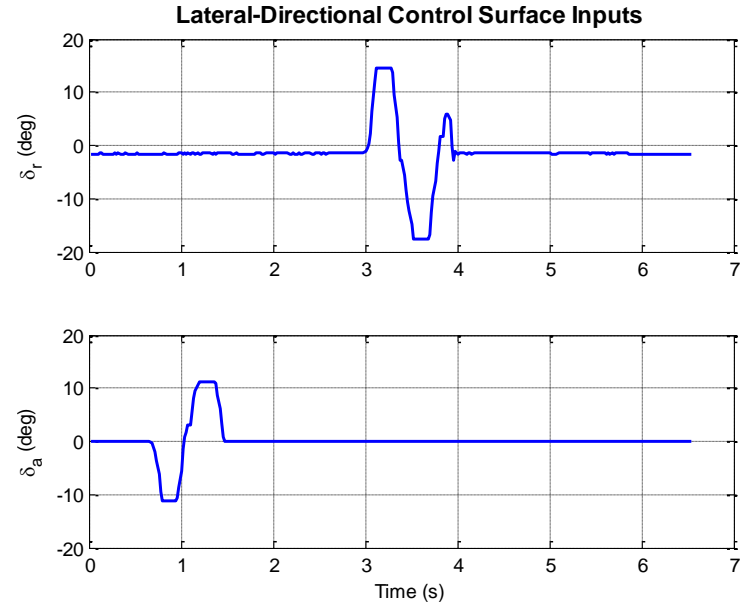

Figure 73: Measured Rudder and Aileron (Right) Deflections used for Optimization
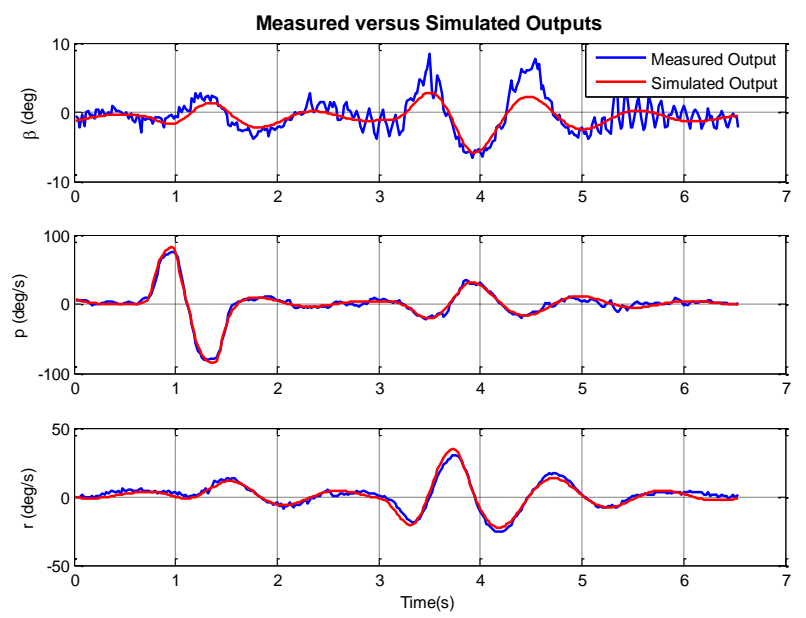

Figure 75: Optimization Results for Sideslip, Roll Rate, and Yaw Rate

The optimized longitudinal and lateral-directional linear models were identified as shown in Equations 5.14 and 5.15, respectively. The corresponding eigenvalues along with the damping ratio, natural frequency, and time constant values for the dynamic modes are listed in Table 33.

$$
\begin{gathered}
{\left[\begin{array}{c}
\dot{\alpha} \\
\dot{q} \\
\dot{\theta}
\end{array}\right]=\left[\begin{array}{ccc}
-6.535 & 4.108 & 0 \\
-15.104 & -2.796 & 0 \\
0 & 1 & 0
\end{array}\right]\left[\begin{array}{l}
\alpha \\
q \\
\theta
\end{array}\right]+\left[\begin{array}{c}
2.926 \\
-26.793 \\
0
\end{array}\right] \delta_{e}} \\
{\left[\begin{array}{c}
\dot{\beta} \\
\dot{p} \\
\dot{r} \\
\dot{\phi}
\end{array}\right]=\left[\begin{array}{cccc}
-0.039 & 0.052 & -0.999 & 0 \\
-51.100 & -11.964 & 8.973 & 0 \\
21.139 & 0.982 & -0.959 & 0 \\
0 & 1 & 0 & 0
\end{array}\right]\left[\begin{array}{l}
\beta \\
p \\
r \\
\phi
\end{array}\right]+\left[\begin{array}{cc}
-0.410 & -0.219 \\
94.313 & 6.578 \\
-1.863 & -6.678 \\
0 & 0
\end{array}\right]\left[\begin{array}{l}
\delta_{a} \\
\delta_{r}
\end{array}\right]}
\end{gathered}
$$


Table 33: Eigenvalues, Damping, and Natural Frequencies of the Optimized PACT Aircraft Model

\begin{tabular}{|c|c|c|c|}
\hline Dynamic Mode & Eigenvalues & Damping & $\begin{array}{c}\text { Natural Frequency } \\
(\mathbf{r a d} / \mathbf{s})\end{array}$ \\
\hline Short Period & $-4.67 \pm 7.65 \mathrm{i}$ & 0.52 & 8.96 \\
\hline Dutch Roll & $-0.44 \pm 4.00 \mathrm{i}$ & 0.11 & 4.02 \\
\hline Roll & -12.07 & - & - \\
\hline
\end{tabular}

The optimized PACT linear state space models will be utilized in the flight control law design for future autonomous flight tests. Additionally, the optimized state space model served as the starting point for the stability and control derivative identification process.

\subsubsection{WVU PACT Stability and Control Derivative Identification}

A detailed approximation of the aircraft inertial characteristics was required for the estimation of the stability and control derivatives. In order to obtain an accurate estimate of the inertial characteristics, a computer-aided design (CAD) model was created using SolidWorks ${ }^{\circledR}$. Each component of the aircraft was modeled to match the dimensions and weight of the actual components they were representing. These parts were collected into an assembly in which each part was located respectively to its physical counterpart. Figure 76 shows the CAD model of the PACT aircraft.

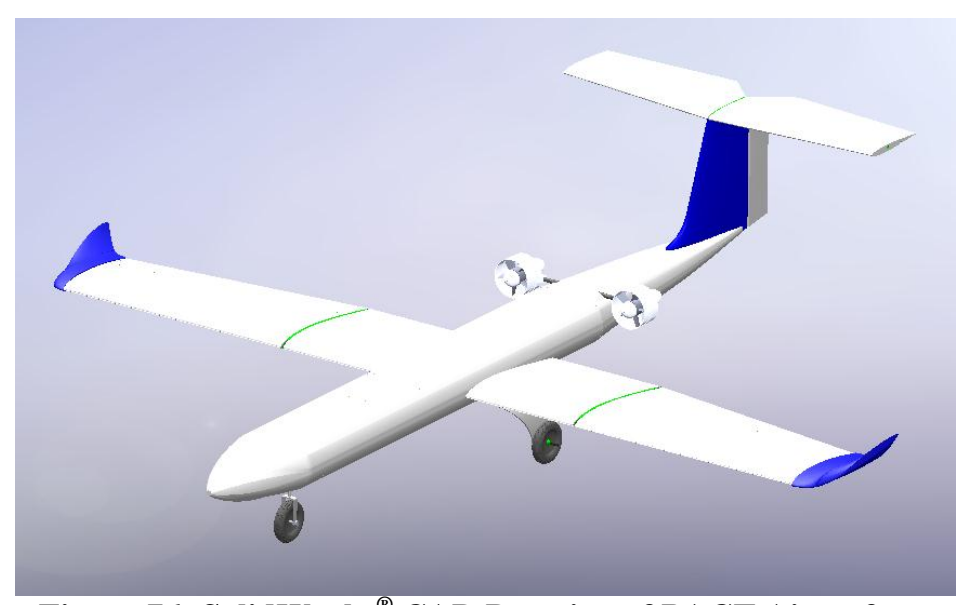

Figure 76: SolidWorks ${ }^{\circledR}$ CAD Drawing of PACT Aircraft

The accuracy of the final assembly was verified by direct measurement of the physical aircraft and comparing it to the aircraft model. These measurements included various dimensions such as wing span and fuselage length, as well as total aircraft weight. Using a tool within SolidWorks ${ }^{\circledR}$, the inertial properties of the aircraft, including the center of gravity, moments of 
inertia, and products of inertia, were found. Table 34 shows the estimated moments of inertia for the PACT.

Table 34: Estimated Moments of Inertia for WVU PACT
\begin{tabular}{|c|c|}
\hline $\begin{array}{c}\text { Moment of } \\
\text { Inertia }\end{array}$ & $\begin{array}{c}\text { Estimated Value } \\
\left(\mathbf{k g ~ m}^{\mathbf{2}}\right)\end{array}$ \\
\hline $\boldsymbol{I}_{x \boldsymbol{x}}$ & 1.41 \\
\hline $\boldsymbol{I}_{\boldsymbol{y}}$ & 3.45 \\
\hline $\boldsymbol{I}_{z z}$ & 2.14 \\
\hline
\end{tabular}

The next step was to determine the aircraft aerodynamic derivatives by converting the optimized linear state space model to provide the initial stability and control derivatives for optimization. Using the values of the geometric and inertial parameters obtained experimentally, it was possible to evaluate the initial values for each of the aerodynamic derivatives from the linear state space model.

The optimization procedure for the PACT aircraft was the same as utilized for the WVU YF22, and again DATCOM estimates were applied. Preliminary trials without the use of DATCOM for estimate of bounds resulted in several stability derivatives optimizing to values that did not make physical sense, including the following:

$$
\begin{aligned}
& c_{L_{\alpha}}=7.59>2 \pi \\
& c_{L_{q}}=-488.34
\end{aligned}
$$

With the application of DATCOM-inspired values for upper and lower optimization bounds, estimates of the stability and control derivatives were improved. The stability and control derivative optimization results are detailed in Section 5.2.3, which includes the specific contributions of DATCOM.

\subsubsection{WVU PACT DATCOM Analysis}

DATCOM was utilized for the estimation of several important stability and control derivatives for the WVU PACT. The stability and control derivative estimation was simplified for the PACT aircraft as compared for the WVU YF-22 configuration; however, assumptions concerning the down-scaling of parameters were required. Mach number and several geometric assumptions concerning the fuselage and wing parameters forced extrapolation of DATCOM data. These estimates were utilized again for optimization purposes with the output error method described in section 5.1.2. For the complete description of the calculation of selected longitudinal and lateral-directional stability and control derivatives for the PACT, please see Appendix A. 
From these estimated values and using engineering judgment, upper and lower bounds were implemented into the stability and control optimization process. In addition to adding upper and lower bounds to the estimated parameters, physical bounds were also incorporated on the drag terms to prevent them from optimizing to negative numbers. Because the DATCOM estimates required several assumptions that could propagate error as calculations progressed, these estimated values were used "loosely". Table 35 summarizes the PID and DATCOM estimated values, the error between the estimates, and the lower and upper bounds applied during optimization.

Table 35: PID and DATCOM Estimate Comparison for WVU PACT

\begin{tabular}{|c|c|c|c|c|c|c|}
\hline Derivative & \begin{tabular}{|c} 
PID Estimated \\
Values
\end{tabular} & $\begin{array}{c}\text { DATCOM } \\
\text { Estimated Values }\end{array}$ & $\%$ Error & $\begin{array}{l}\text { Applied } \\
\text { Lower } \\
\text { Bounds }\end{array}$ & $\begin{array}{l}\text { Applied } \\
\text { Upper } \\
\text { Bounds }\end{array}$ & Sign Convention \\
\hline$c_{D_{0}}$ & 0.009 & 0.010 & 14.943 & 0.001 & 0.02 & positive \\
\hline$c_{D_{\alpha}}$ & 0.479 & - & & - & - & positive \\
\hline$c_{D_{q}}$ & 0 & 0 & & - & - & positive \\
\hline$c_{D_{\delta e}}$ & 0.090 & - & & - & - & positive \\
\hline$c_{L_{0}}$ & -0.084 & - & & - & - & either \\
\hline$c_{L_{\alpha}}$ & 2.888 & 3.580 & 23.961 & 1.00 & 5.37 & positive \\
\hline$c_{L_{q}}$ & 1.370 & - & & - & - & positive \\
\hline$c_{L_{\delta e}}$ & 0.221 & 0.242 & 9.569 & 0.10 & 0.48 & positive \\
\hline$c_{m_{0}}$ & 0.155 & - & & - & - & either \\
\hline$c_{m_{\alpha}}$ & -1.206 & -4.776 & 295.987 & 1.00 & 6.00 & either \\
\hline$c_{m_{q}}$ & -13.953 & -13.606 & 2.487 & -20.41 & -6.80 & negative \\
\hline$c_{m_{\delta e}}$ & -0.670 & -0.647 & 3.432 & -0.97 & -0.32 & negative \\
\hline$c_{Y_{0}}$ & -0.014 & 0 & & - & - & 0 \\
\hline$c_{Y_{\beta}}$ & -0.267 & - & & - & - & negative \\
\hline$c_{Y_{p}}$ & -0.574 & - & & - & - & negative \\
\hline$c_{Y_{r}}$ & 0.030 & - & & - & - & positive \\
\hline$c_{Y_{\delta a}}$ & -0.015 & 0 & & - & - & 0 \\
\hline$c_{Y_{\delta r}}$ & 0.531 & 0.506 & 4.616 & 0.10 & 1.01 & positive \\
\hline$c_{l_{0}}$ & -0.020 & 0 & & - & - & 0 \\
\hline$c_{l_{\beta}}$ & -0.408 & -0.418 & 2.302 & -0.63 & -0.21 & negative \\
\hline$c_{l_{p}}$ & -2.703 & -2.207 & 18.341 & -3.31 & -1.10 & negative \\
\hline
\end{tabular}




\begin{tabular}{|c|c|c|c|c|c|c|}
\hline$c_{l_{r}}$ & 0.197 & - & & - & - & positive \\
\hline$c_{l_{\delta a}}$ & 0.647 & 0.220 & 66.095 & 0.10 & 1.00 & positive \\
\hline$c_{l_{\delta r}}$ & 0.113 & - & & - & - & positive \\
\hline$c_{n_{0}}$ & 0.023 & 0 & & - & - & 0 \\
\hline$c_{n_{\beta}}$ & 0.216 & 0.532 & 146.068 & 0.10 & 0.80 & positive \\
\hline$c_{n_{p}}$ & 0.096 & - & & - & - & either \\
\hline$c_{n_{r}}$ & -0.561 & -0.558 & 0.428 & -0.84 & -0.28 & negative \\
\hline$c_{n_{\delta a}}$ & -0.008 & - & & - & - & negative \\
\hline$c_{n_{\delta r}}$ & -0.010 & - & & - & - & negative \\
\hline
\end{tabular}

The static margin - a non-dimensional distance of the neutral point of the aircraft behind the center of gravity - was estimated through PID to be 0.418 . The DATCOM estimated values did not produce a similar result, due to the high estimation of $c_{m_{\alpha}}$. Figures 77 and 78 show the optimization results for the longitudinal states during an elevator doublet.

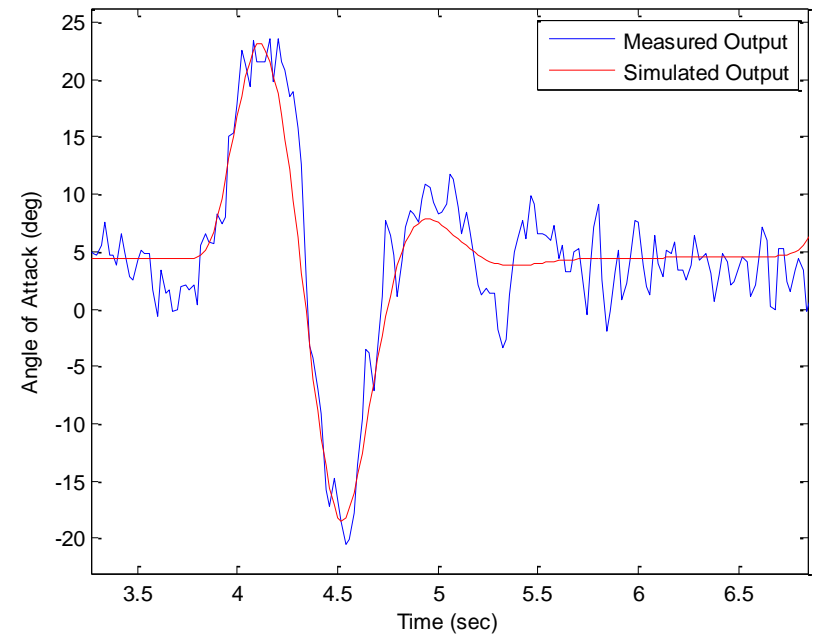

Figure 77: PACT Optimization Result for Angle of Attack during Elevator Doublet

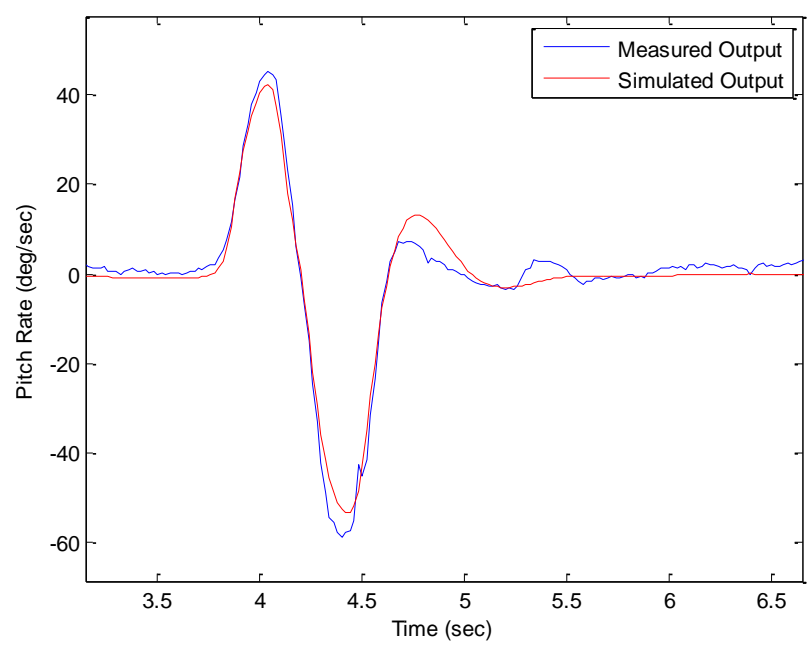

Figure 78: PACT Optimization Result for Pitch Rate during Elevator Doublet

Figures 79 and 80 show the optimization results for the roll and yaw rates, respectively. The segments of flight data shown are for two maneuvers performed on the lateral-directional control surfaces, specifically an aileron doublet followed by a rudder doublet. 


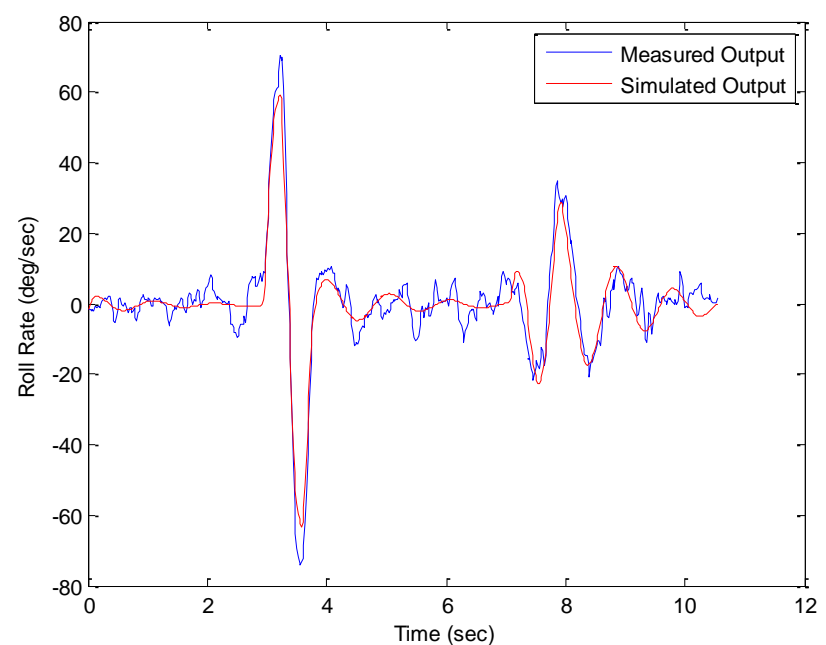

Figure 79: PACT Optimization Result for Roll Rate during Aileron and Rudder Doublets

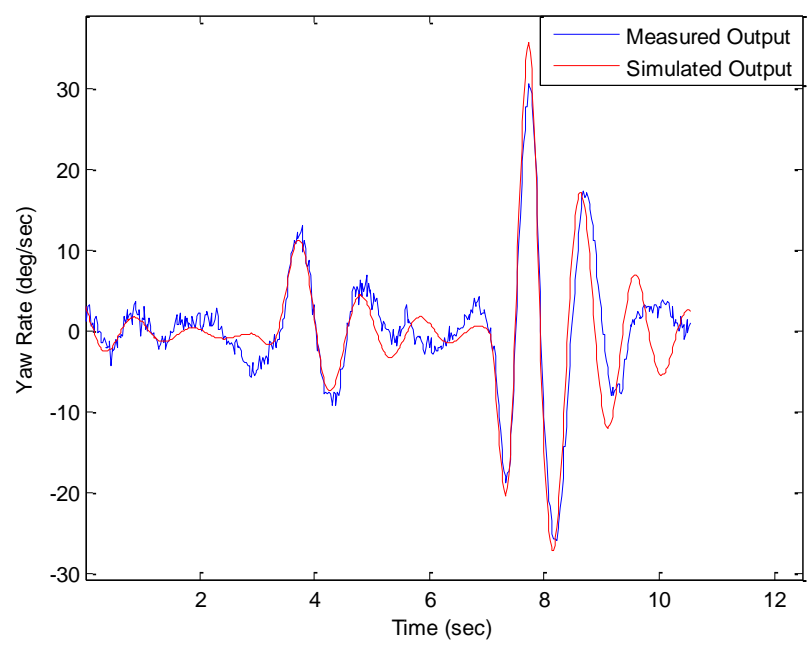

Figure 80: PACT Optimization Result for Yaw Rate during Aileron and Rudder Doublets

A lift-to-drag ratio analysis was conducted from the estimated stability and control derivatives representing lift and drag on the WVU PACT. Table 36 summarizes the estimated lift and drag coefficients and the lift-to-drag ratio as predicted through PID.

Table 36: Lift to Drag Ratio Analysis from PID Values for PACT

\begin{tabular}{|c|c|c|c|c|c|c|c|c|c|}
\hline Aircraft & $c_{D_{0}}$ & $c_{D_{\alpha}}$ & $c_{L_{0}}$ & $c_{L_{\alpha}}$ & $c_{L_{q}}$ & $c_{L_{\delta e}}$ & $C_{D}$ & $C_{L}$ & $\boldsymbol{L} / \boldsymbol{D}$ \\
\hline WVU PACT & 0.009 & 0.480 & -0.084 & 2.888 & 1.370 & 0.221 & 0.488 & 4.395 & $\mathbf{9 . 0 0}$ \\
\hline
\end{tabular}

These estimated values were implemented into a simulator and several simulation studies were performed for validation.

\subsubsection{WVU PACT Simulation Studies}

Once the stability and control derivatives were optimized under nominal flight conditions, simulation studies were conducted with separate flight data sets to validate the identified model. Figures 81 and 82 show a segment of flight data where an elevator doublet was performed. The measured flight data was compared to the simulated output given the same elevator input with the resulting angle of attack and pitch rates. Figures 83 and 84 represent two different maneuvers: roll rate response due to an aileron doublet and yaw rate response due to an aileron doublet followed closely by a rudder doublet. The measured flight data and simulated output are shown for the roll rate and yaw rate responses. 


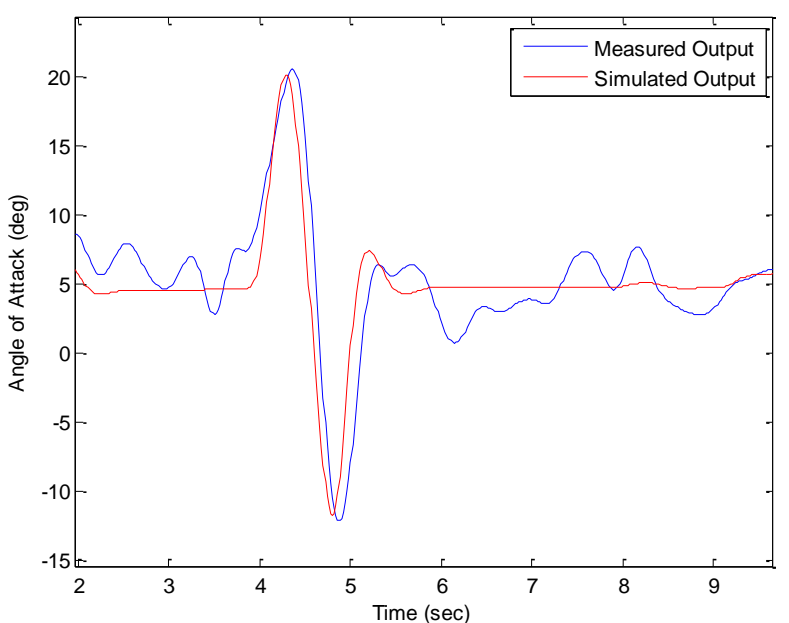

Figure 81: Simulation Results for Angle of Attack with Elevator Doublet

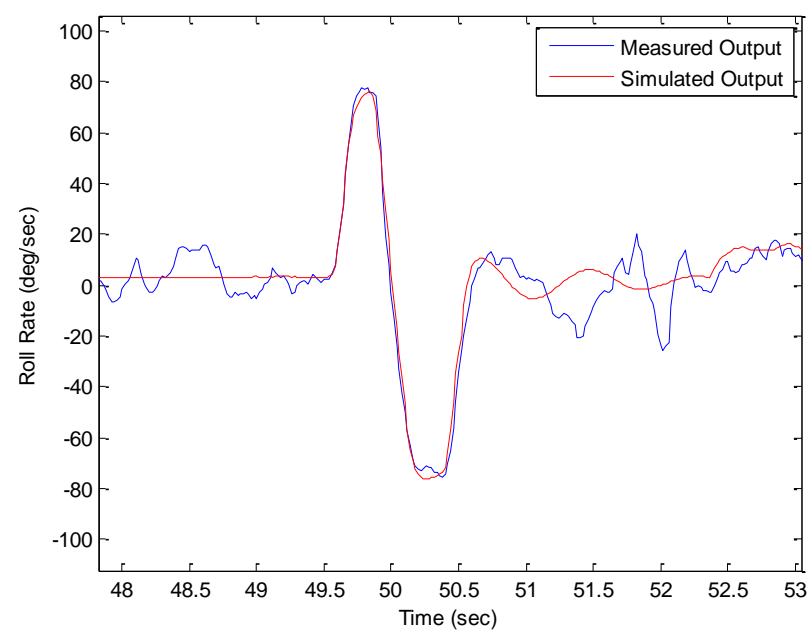

Figure 83: Simulation Results for Roll Rate with Elevator Doublet

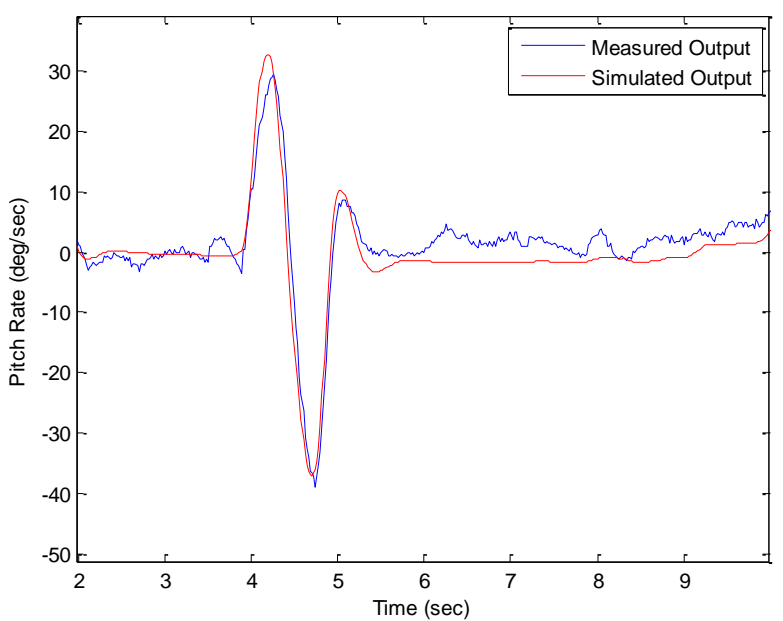

Figure 82: Simulation Results for Pitch Rate with Elevator Doublet

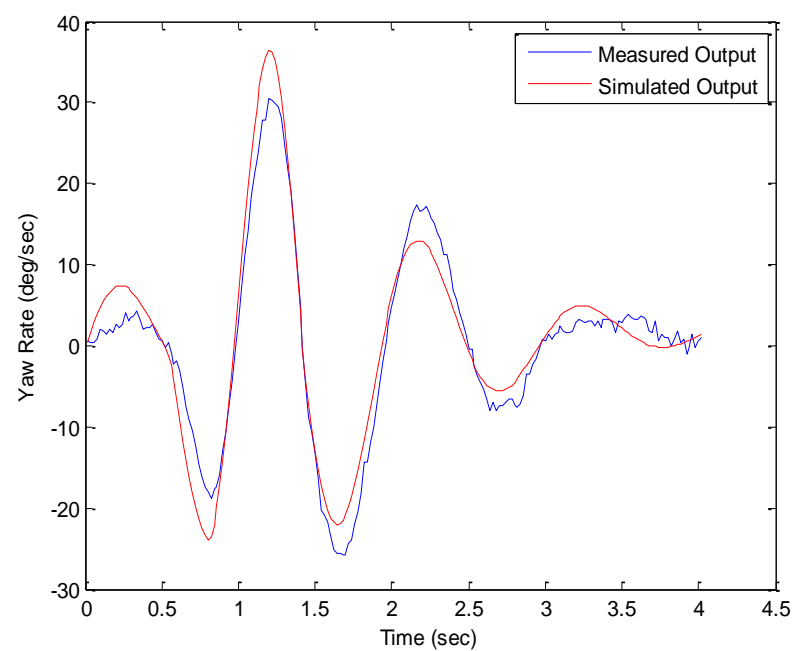

Figure 84: Simulation Results for Yaw Rate with Aileron Doublet

An error analysis was conducted to determine the average error between selected measured states and the simulated state outputs. For selected segments of flight data, the mean error and standard deviation were analyzed to provide metrics for the validity of the identified aircraft mathematical model. Table 37 provides a summary of typical maneuvers and output states that were analyzed for average error and standard deviation. 
Table 37: Error Analysis of Measured Flight Data and Simulated Model Output

\begin{tabular}{|c|c|c|c|c|c|c|c|c|}
\hline & \multicolumn{2}{|c|}{$\begin{array}{c}\text { Angle of Attack } \\
\text { (deg) }\end{array}$} & \multicolumn{2}{c|}{$\begin{array}{c}\text { Pitch Rate } \\
\text { (deg/sec) }\end{array}$} & \multicolumn{2}{c|}{ Roll Rate (deg/sec) } & \multicolumn{2}{c|}{$\begin{array}{c}\text { Yaw Rate } \\
\text { (deg/sec) }\end{array}$} \\
\hline Maneuver & $\begin{array}{c}\text { Mean } \\
\text { Error }\end{array}$ & $\begin{array}{c}\text { Standard } \\
\text { Deviation }\end{array}$ & $\begin{array}{c}\text { Mean } \\
\text { Error }\end{array}$ & $\begin{array}{c}\text { Standard } \\
\text { Deviation }\end{array}$ & $\begin{array}{c}\text { Mean } \\
\text { Error }\end{array}$ & $\begin{array}{c}\text { Standard } \\
\text { Deviation }\end{array}$ & $\begin{array}{c}\text { Mean } \\
\text { Error }\end{array}$ & $\begin{array}{c}\text { Standard } \\
\text { Deviation }\end{array}$ \\
\hline $\begin{array}{c}\text { Nominal } \\
\text { Elevator }\end{array}$ & 0.186 & 2.262 & 0.421 & 2.923 & - & - & - & - \\
\hline $\begin{array}{c}\text { Nominal } \\
\text { Aileron }\end{array}$ & - & - & - & - & 0.119 & 4.820 & 0.089 & 1.894 \\
\hline $\begin{array}{c}\text { Nominal } \\
\text { Rudder }\end{array}$ & - & - & - & - & 1.222 & 5.217 & 0.625 & 3.711 \\
\hline
\end{tabular}

The average error and standard deviation of the maneuvers performed were averaged from three maneuvers. There was a flight data restriction for the PACT modeling since there were only two recorded flights at the time of model identification; therefore, only a few maneuvers were available for this analysis.

Based on the simulation studies and error analysis, it was determined that the identified model of the WVU PACT performs as expected. The longitudinal and lateral-directional dynamic responses were accurately represented, and it is expected that this model will meet expectations for use within a flight control system.

\subsubsection{WVU PACT Confidence Interval Analysis}

A confidence interval analysis was conducted on the state space parameters through the optimization process. This provided a predicted percent error calculation and a $95 \%$ confidence interval for the identified parameters in the PACT state space model. The parameters were separately optimized for the longitudinal and lateral-directional dynamics, thus the confidence intervals were also separately evaluated.

The identified parameters for the longitudinal state space model include values in the state and input matrices that affect angle of attack and pitch rate. The parameters are identified based on their positions in the state or input matrix as follows:

$$
\left[\begin{array}{c}
\dot{\alpha} \\
\dot{q}
\end{array}\right]=\left[\begin{array}{ll}
A(1,1) & A(1,2) \\
A(2,1) & A(2,2)
\end{array}\right]\left[\begin{array}{l}
\alpha \\
q
\end{array}\right]+\left[\begin{array}{c}
B(1) \\
B(2)
\end{array}\right]\left[\delta_{e}\right]
$$

Table 38 shows the results of the confidence interval analysis for the longitudinal state space parameters. The segment of flight data used for optimization was also used to determine the $95 \%$ confidence intervals and error analysis. The standard error is equivalent to the standard deviation 
of the sample mean based on the population mean (i.e. the measured flight data used for optimization). The $95 \%$ confidence intervals for each estimated parameter are based on the sample flight data used for optimization. The confidence intervals provide an estimated value range for the flight data and where the parameter estimate should fall.

Table 38: PACT Longitudinal State Space Estimates, Error Analyses, and 95\% Confidence Intervals

\begin{tabular}{|c|c|c|c|c|c|}
\hline Parameter & Estimate & $\begin{array}{c}\text { Standard } \\
\text { Error }\end{array}$ & \% Error & $\begin{array}{c}\text { Confidence } \\
\text { Interval } \\
\text { (Lower) }\end{array}$ & $\begin{array}{c}\text { Confidence } \\
\text { Interval } \\
\text { (Upper) }\end{array}$ \\
\hline $\mathbf{A}(\mathbf{1 , 1})$ & -6.535 & 0.248 & 3.80 & -7.030 & -6.040 \\
\hline $\mathbf{A}(\mathbf{1 , 2})$ & 4.108 & 0.161 & 3.90 & 3.785 & 4.43 \\
\hline $\mathbf{A}(\mathbf{2 , 1})$ & -15.104 & 0.490 & 3.20 & -16.085 & -14.124 \\
\hline $\mathbf{A}(\mathbf{2 , 2})$ & -2.796 & 0.278 & 9.90 & -3.351 & -2.241 \\
\hline $\mathbf{B}(\mathbf{1})$ & 2.926 & 0.456 & 15.60 & 2.014 & 3.838 \\
\hline $\mathbf{B}(\mathbf{2})$ & -26.793 & 0.657 & 2.50 & -28.107 & -25.478 \\
\hline
\end{tabular}

The identified parameters for the lateral-directional state space model include values in the state and input matrices that affect sideslip, roll rate, and yaw rate. The parameters are identified based on their positions in the state or input matrix as follows:

$$
\left[\begin{array}{c}
\dot{\beta} \\
\dot{p} \\
\dot{r}
\end{array}\right]=\left[\begin{array}{lll}
A(1,1) & A(1,2) & A(1,3) \\
A(2,1) & A(2,2) & A(2,3) \\
A(3,1) & A(3,2) & A(3,3)
\end{array}\right]\left[\begin{array}{l}
\beta \\
p \\
r
\end{array}\right]+\left[\begin{array}{ll}
B(1,1) & B(1,2) \\
B(2,1) & B(2,2) \\
B(3,1) & B(3,2)
\end{array}\right]\left[\begin{array}{l}
\delta_{a} \\
\delta_{r}
\end{array}\right]
$$

Table 39 shows the results of the confidence interval analysis for the lateral-directional state space parameters. The segment of flight data used for optimization was also used to determine the $95 \%$ confidence intervals and error analysis.

Table 39: PACT Lateral-Directional State Space Estimates, Error Analyses, and 95\% Confidence Intervals

\begin{tabular}{|c|c|c|c|c|c|}
\hline Parameter & Estimate & $\begin{array}{c}\text { Standard } \\
\text { Error }\end{array}$ & \% Error & $\begin{array}{c}\text { Confidence } \\
\text { Interval } \\
\text { (Lower) }\end{array}$ & $\begin{array}{c}\text { Confidence } \\
\text { Interval } \\
\text { (Upper) }\end{array}$ \\
\hline $\mathbf{A}(\mathbf{1 , 1})$ & -0.034 & 0.038 & 111.30 & -0.109 & 0.042 \\
\hline $\mathbf{A}(\mathbf{2 , 1})$ & -51.100 & 2.579 & 5.00 & -56.257 & -45.942 \\
\hline $\mathbf{A}(\mathbf{2 , 2})$ & -11.964 & 0.406 & 3.40 & -12.777 & -11.152 \\
\hline $\mathbf{A}(\mathbf{2 , 3})$ & 8.973 & 0.394 & 4.40 & 8.186 & 9.761 \\
\hline $\mathbf{A}(\mathbf{3 , 1})$ & 21.139 & 0.197 & 0.90 & 20.745 & 21.532 \\
\hline $\mathbf{A}(\mathbf{3 , 3})$ & -0.959 & 0.055 & 5.80 & -1.069 & -0.848 \\
\hline $\mathbf{B}(\mathbf{1 , 2})$ & -0.219 & 0.035 & 16.20 & -0.289 & -0.148 \\
\hline $\mathbf{B}(\mathbf{2 , 1})$ & 94.313 & 2.932 & 3.10 & 88.452 & 100.180 \\
\hline $\mathbf{B}(\mathbf{2 , 2})$ & 6.578 & 1.101 & 16.70 & 4.376 & 8.781 \\
\hline $\mathbf{B}(\mathbf{3 , 1})$ & -1.863 & 0.158 & 8.50 & -2.178 & -1.547 \\
\hline $\mathbf{B}(\mathbf{3 , 2})$ & -6.678 & 0.158 & 2.40 & -6.995 & -6.362 \\
\hline
\end{tabular}


The standard error and percent error estimates for the identified longitudinal and lateraldirectional state space parameters were relatively low with only a few terms having a high error percentage and wider confidence interval. One longitudinal input matrix estimate exceeded $15 \%$ error, and three parameters in the lateral-directional state space model exceeded $16 \%$ error. One parameter affecting sideslip estimation $A(1,1)$ for the lateral-directional parameters had an error exceeding $100 \%$. This is due to the lower excitation of this state in the optimization data set, which made it more difficult to clearly identify. These results indicate that based on the measured flight data used for optimization, the estimated parameters will accurately represent what was observed in flight. Following this analysis, this identified state space model was found to be sufficient to be utilized for flight control law design. 


\section{Summary and Conclusions}

A parameter identification study from flight data was conducted on two research platforms: the WVU YF-22 and PACT. For the WVU YF-22, a state space representation under nominal and primary control surface failure conditions was estimated for use within the design of a faulttolerant flight control system. Stability and control derivatives were also estimated under nominal and primary control surface failure conditions for use within a flight simulator to test flight control laws prior to actual flight testing on the aircraft. For the WVU PACT, a state space representation under healthy aircraft conditions was estimated for use in the design of fight

control laws. Again, stability and control derivatives were estimated to incorporate into a simulator to test flight control laws before performing actual experiments.

For the state space estimation, the Matlab ${ }^{\circledR}$ System Identification Toolbox was used to evaluate the maneuvers and the aircraft system dynamic responses recorded in flight. After evaluating the eigenvalues, damping ratios, and natural frequencies from the maneuvers, a state space model was selected to serve as the set of initial values for an output error optimization scheme. The optimization scheme used a set of actual flight data - input maneuvers and measured state outputs - to estimate the state space parameters through a comparison between the recorded flight data and the simulated output. This optimization focused on the angle of attack, pitch rate, roll rate, and yaw rate outputs from the simulated model and a confidence interval analysis was conducted on the final estimated parameters. These final estimated parameters served as the nominal components of YF-22 CoDICE model used for fault-tolerant flight control law design and as the PACT model. To identify the cross-coupling terms of the input matrix within the CoDICE model, the FTR method was employed. Segments of flight data with primary control surface failures were utilized to model the cross-coupling dynamics of the YF-22 aircraft.

For the stability and control derivative estimation, the state space models were converted to initial estimates using the geometric and inertial properties of the two aircraft platforms. These values were optimized using the output error method with sets of measured flight data. Through attempts at optimizing the stability and control derivatives, it became necessary to incorporate upper and lower bounds on some of the parameters. To not blindly estimate these optimization bounds, USAF DATCOM was utilized as a tool for estimation of some of the stability and control derivatives for the WVU YF-22 and PACT research platforms. Because DATCOM was 
not intended for small aircraft with lower flight speeds $($ Mach $<0.2)$, extrapolation of the data on the charts became necessary. It was also necessary to incorporate several geometric assumptions for the YF-22 body, which is not a conventional cylindrical fuselage. Through these estimations, error may have propagated through the calculations that cannot be easily quantified. It is because of these assumptions that the stability and control derivative estimations from DATCOM were applied loosely when being used as optimization constraints. Although it was necessary to apply physical bounds to the optimization process because drag values were being identified as negative and $c_{L_{\alpha}}$, for example, was exceeding values that made physical sense based on engineering knowledge and judgment.

For both the WVU YF-22 and PACT platforms, the nominal stability and control derivatives were optimized successfully using DATCOM estimates as guidelines for upper and lower parameter bounds. For the cross-coupled control derivatives for the WVU YF-22, upper and lower bounds were also necessary for the optimization process. As segments of flight data under control surface failure conditions were implemented in this optimization, bounds based on the control derivative values obtained for the nominal control derivatives were used.

Following the WVU YF-22 and PACT parameter estimation, simulation studies were conducted with different sets of flight data than were used for identification. Measured control surface input maneuvers were passed through the identified models and the simulated output was compared to the measured output from the flight data. An error analysis was conducted over several of the maneuvers and an average error and average standard deviation for several of the states was observed. Based on the low average error and standard deviations for the selected states, it was determined that the estimated models for the WVU YF-22 and PACT were accurate.

The successful development of flight control laws rely on accurate modeling of the aircraft under nominal and actuator failure conditions. Actuator failure detection, identification, and accommodation relies on the modeling of the dynamic properties of the aircraft under locked actuator conditions to maintain safe flight on the aircraft. Thus, this study aimed to accurately model the two aircraft platforms under nominal and actuator failure conditions as the first step within the development of a set of fault-tolerant flight control laws. In order to obtain an accurate simulator to test the flight control laws, the stability and control derivatives were optimized based on the aircraft flight performance. Although these identified parameters are not "true" 
values, they are estimates that must reflect the dynamic behavior of the aircraft. It is desirable that these estimated parameters also make physical sense, to increase the credibility of the identified model. This modeling study showed that it may become necessary to apply constraints on the stability and control derivative optimization process, and it showed that USAF DATCOM estimates may be used to implement the upper and lower parameter bounds. This process was applied more as an art than science, as it was through trial and error that it became evident which parameters required the constraints and how they would affect the other optimizing parameters. It was shown that through this application of DATCOM estimates for the bounds on selected parameters, the optimization for two different research platforms was significantly improved, thus improving the simulator fidelity for flight control law testing.

In addition to utilizing DATCOM to improve the optimization process for the stability and control derivatives, there was uniqueness in the combination and successful implementation of the different PID methods. The output-error and Fourier Transform Regression methods have been proven for PID purposes and have been successfully implemented in off-line studies. The combination of these methods were used to obtain accurate models from flight data of the two research platforms for state space and stability and control derivative estimates; the addition of DATCOM to this identification process further improved the parameter estimates and the final product of this research. 


\section{Appendix A: DATCOM Calculation}

\section{DATCOM Calculations for WVU YF-22}

\subsubsection{WVU YF-22 Downwash Calculation}

The downwash parameter $\frac{d \varepsilon}{d \alpha}$ was calculated using Equation 7.1:

$$
\frac{d \varepsilon}{d \alpha}=4.44\left(K_{A R} K_{\lambda} K_{H} \sqrt{\cos \Lambda_{c / 4}}\right)^{1.19}
$$

where:

$$
\begin{gathered}
K_{A R}=\frac{1}{A R}-\frac{1}{1+A R^{1.7}} \\
K_{\lambda}=\frac{10-3 \lambda}{7} \\
K_{H}=\frac{1-\frac{m}{2}}{\sqrt[3]{r}}
\end{gathered}
$$

The parameters represented in Equations 7.2 - 7.4 were determined using Figures 85-87. These parameters represent the effects caused by the wing aspect ratio, wing tip ratio, and the horizontal tail location with respect to the wing.

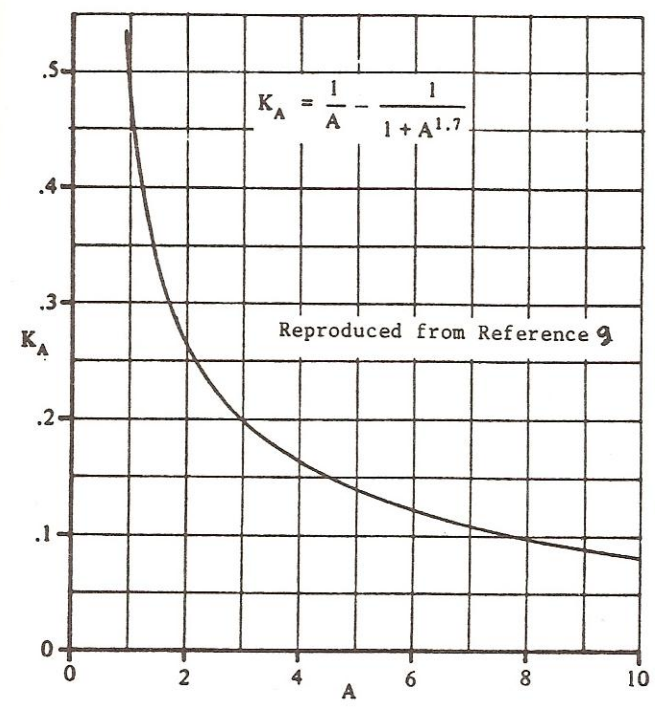

Figure 85: Factor $K_{A}$ used for Calculating Downwash [Reproduced from Reference 42] 


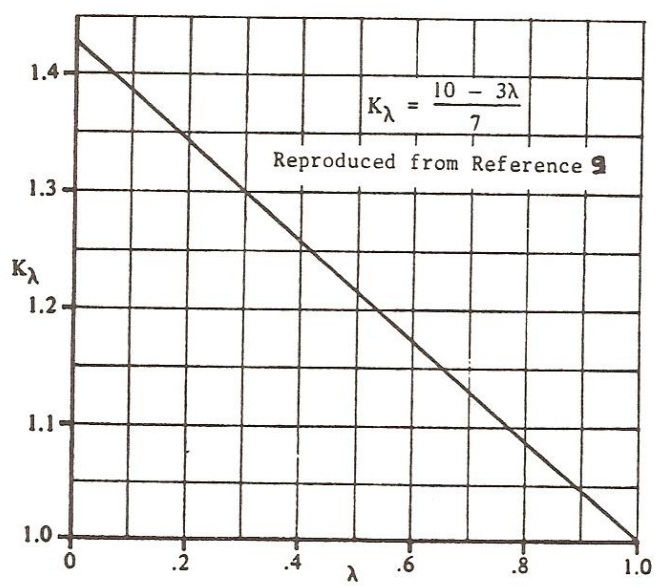

Figure 86: Factor $K_{\lambda}$ used for Calculating Downwash [Reproduced from Reference 42]

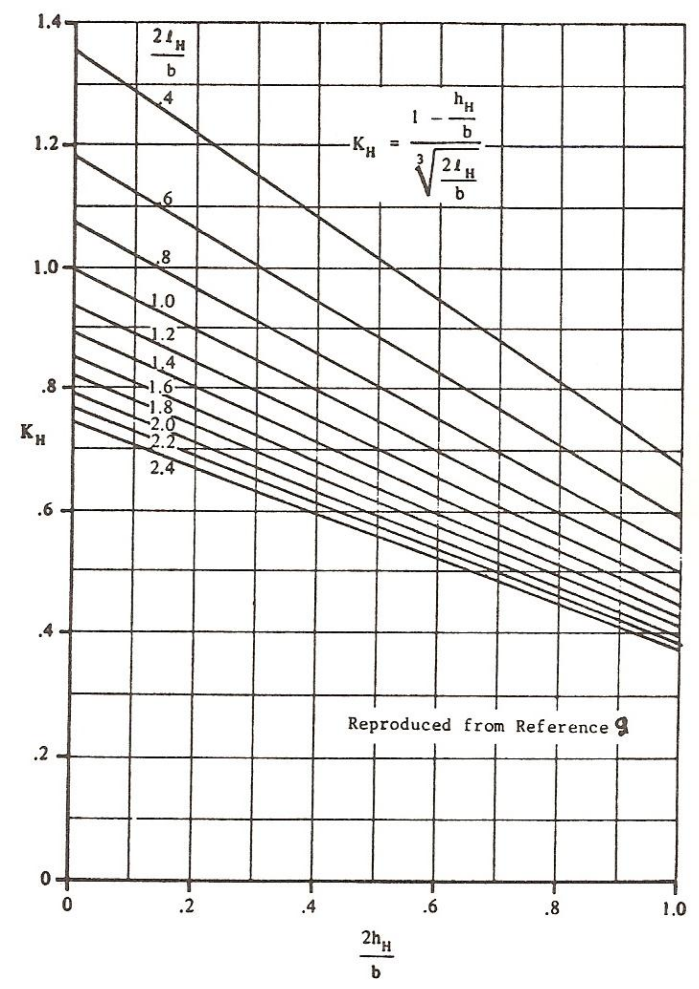

Figure 87: Factor $K_{H}$ used for Calculating Downwash [Reproduced from Reference 42]

The representation of the dynamic pressure difference at the horizontal tail compared to the wing, $\eta_{H}$, was necessary to calculate the downwash and was obtained from Figure 88. 

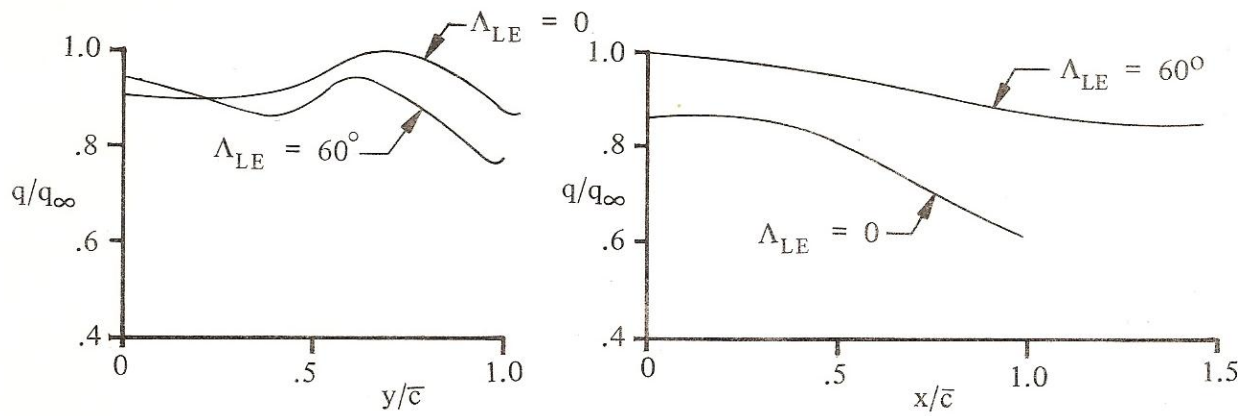

Figure 88: Spanwise (Left) and Longitudinal (Right) Variations in Dynamic Pressure Ratio [Reproduced from Reference 42]

\subsubsection{WVU YF-22 Stability Derivatives (DATCOM)}

The following stability derivatives are the main derivatives that were calculated for the WVU YF-22. The purpose was to obtain estimates for these values to help guide the optimization process for parameter identification.

\subsubsection{Zero-Lift Drag Derivative: $c_{D_{o}}$}

To calculate the wing-body tail contribution to $c_{D_{o}}$, the characteristics of the wing, horizontal tail, and vertical tail were identified. In addition to the wing and tail contributions, several body parameters such as the body length and diameter were required for this calculation. According to DATCOM, eight basic steps should be followed to calculate the wing-body-tail contribution to $c_{D_{o}}$.

Step 1: The skin friction coefficient, $C_{f}$, were calculated for the wing, horizontal and vertical tails, and the body. The Reynolds number of the airflow over the aircraft components was required to determine the skin friction coefficients. The following calculations were completed to determine the Reynolds number of the airflow over each component at a Mach of 0.123 (133.76 $\mathrm{ft} / \mathrm{s}$ ) and with air dynamic viscosity of $1.58 \times 10^{-4} \mathrm{ft}^{2} / \mathrm{s}$. In these equations, $c$ represents the characteristic length of the surface in feet.

$$
\begin{gathered}
\operatorname{Re}_{\text {wing }}=\frac{u c}{v}=\frac{(133.76) 1.7}{1.58 \times 10^{-4}}=1.44 \times 10^{6} \\
\operatorname{Re}_{H-\text { Tail }}=\frac{u c}{v}=\frac{(133.76) 0.958}{1.58 \times 10^{-4}}=8.11 \times 10^{5}
\end{gathered}
$$




$$
\begin{aligned}
\operatorname{Re}_{V-T a i l} & =\frac{u c}{v}=\frac{(133.76) 1.083}{1.58 \times 10^{-4}}=9.17 \times 10^{5} \\
\operatorname{Re}_{b o d y} & =\frac{u c}{v}=\frac{(133.76) 6.83}{1.58 \times 10^{-4}}=5.78 \times 10^{6}
\end{aligned}
$$

Knowing these Reynolds numbers, the skin friction coefficients were calculated at a Mach of 0.123 .

$$
\begin{aligned}
& C_{f_{\text {wing }}}=0.00405 \\
& C_{f_{H-\text { aul }}}=0.0046 \\
& C_{f_{V-\text { tail }}}=0.00408 \\
& C_{f_{\text {body }}}=0.00325
\end{aligned}
$$

Step 2: The skin-friction and pressure-drag contributions of each of the components were calculated. The resulting values were calculated as follows:

Wing: $\quad\left[1+L\left(\frac{t}{c}\right)+100\left(\frac{t}{c}\right)^{4}\right]_{W}=\left[1+1.2(0.098)+100(0.098)^{4}\right]_{W}=1.13$

H-tail: $\quad\left[1+L\left(\frac{t}{c}\right)+100\left(\frac{t}{c}\right)^{4}\right]_{H}=\left[1+1.2(0.087)+100(0.087)^{4}\right]_{H}=1.11$

V-tail: $\quad\left[1+L\left(\frac{t}{c}\right)+100\left(\frac{t}{c}\right)^{4}\right]_{V}=\left[1+1.2(0.058)+100(0.058)^{4}\right]_{V}=1.07$

Body: $\quad\left\lfloor 1+\frac{60}{\left(\frac{l_{b}}{d}\right)^{3}}+0.0025 \frac{l_{b}}{d}\right\rfloor=\left[1+\frac{60}{\left(\frac{82}{12}\right)^{3}}+0.0025\left(\frac{82}{12}\right)\right]=1.21$

Step 3: The lifting-surface correction factors for the wing, horizontal tail, and vertical tail were determined by:

Wing:

$$
\begin{aligned}
& \cos \Lambda_{\left(t / c_{\max }\right.}=\cos \left(26^{\circ}\right)=0.899 \\
& \cos \Lambda_{\left(t / c_{\text {max }}\right.}=\cos \left(25^{\circ}\right)=0.906 \\
& \cos \Lambda_{(t /)_{\max }}=\cos \left(10^{\circ}\right)=0.985
\end{aligned}
$$

H-tail:

V-tail:

Now using Figure 89, the lifting-surface correction factors were determined. 


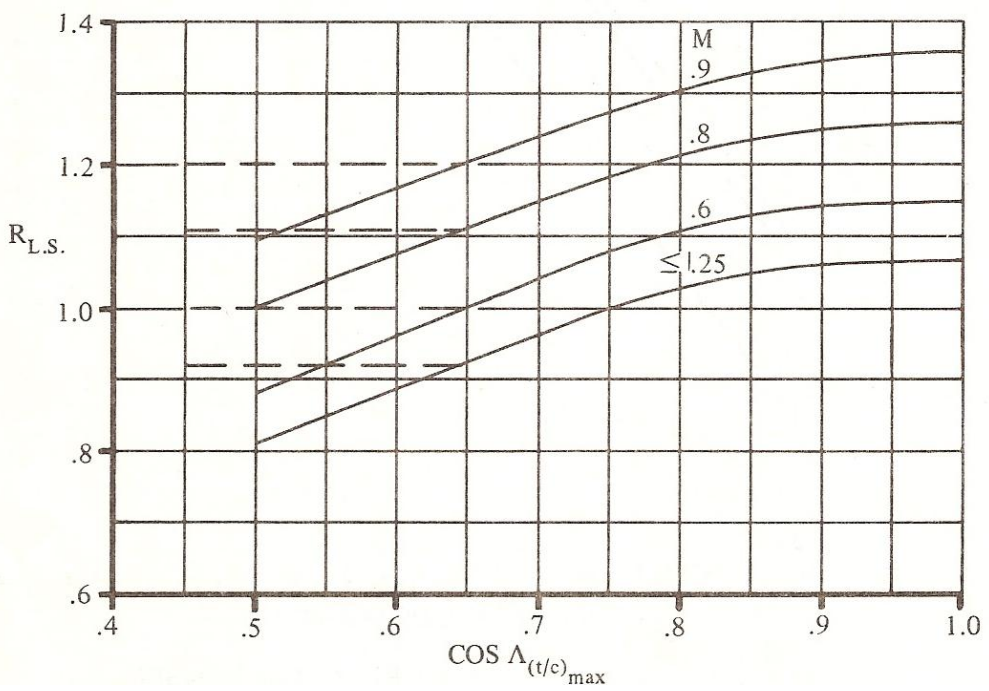

Figure 89: Lifting-Surface Correlation Factor for Subsonic Minimum Drag [Reproduced from Reference 41]

$$
\begin{aligned}
& \left(R_{\text {L.S. }}\right)_{W}=1.06 \\
& \left(R_{\text {L.S. }}\right)_{H}=1.06 \\
& \left(R_{\text {L.S. }}\right)_{V}=1.07
\end{aligned}
$$

Step 4: The wing-body interference correlation factor was determined from Figure 90 using the Reynolds number of the airflow over the fuselage, $R_{W . B .}=1.08$. 


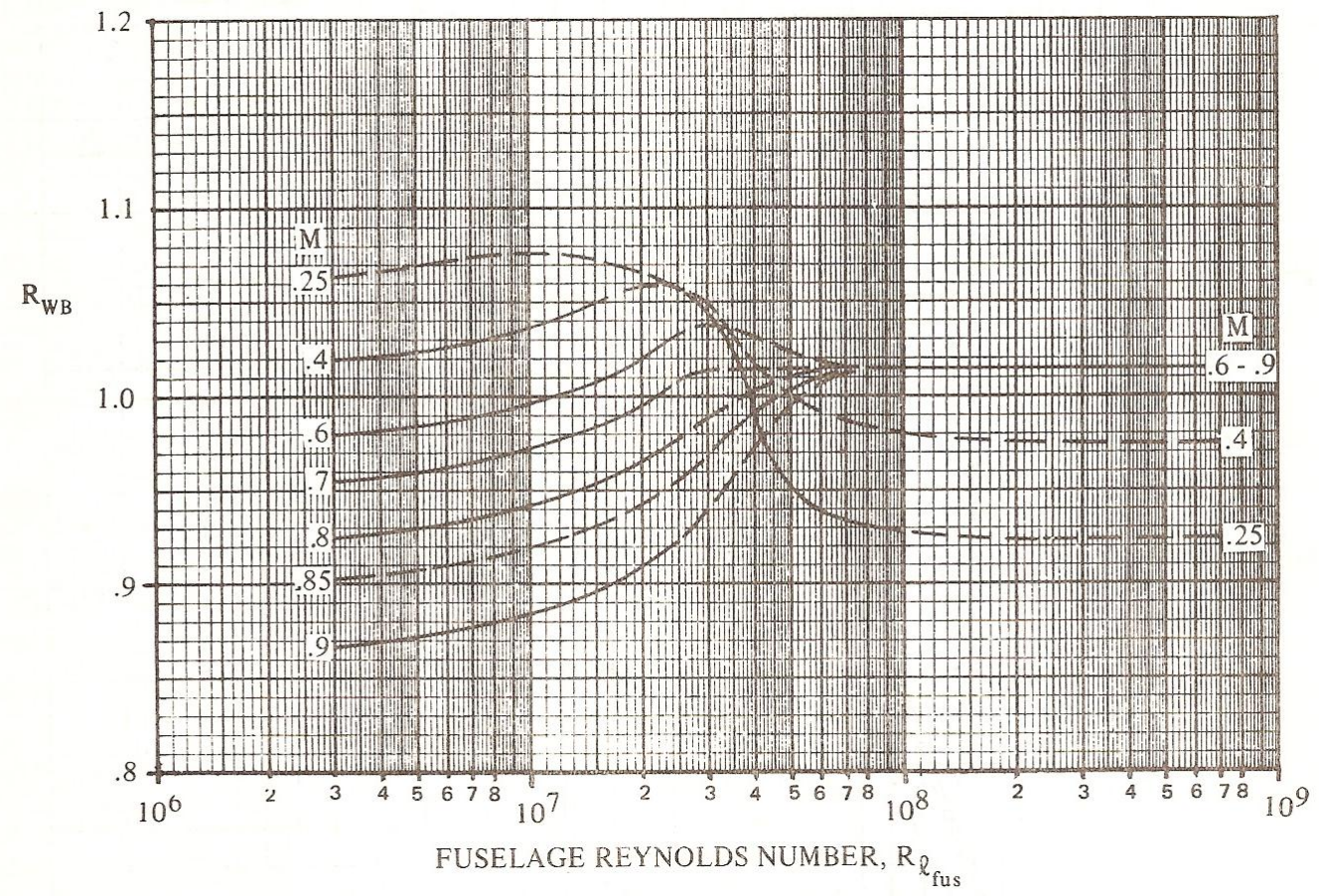

Figure 90: Wing-Body Interference Correlation Factor - Subsonic Speeds [Reproduced from Reference 41]

Step 5: The wing-body zero-lift drag was calculated exclusive of the base drag. All lengths and areas used in Equation 7.5 are in inches and square inches, respectively.

$$
\begin{aligned}
& \left(C_{D_{o}}\right)_{W B}-C_{D_{b}} \frac{S_{B}}{S_{r e f}}= \\
& \quad=\left\{\left(C_{f}\right)_{W}\left[1+L\left(\frac{t}{c}\right)+100\left(\frac{t}{c}\right)^{4}\right]_{W}\left(R_{L . S .}\right)_{W} \frac{\left[\left(S_{w e t}\right)_{W}\right]_{e}}{S_{r e f}}+\left(C_{f}\right)_{B}\left[1+\frac{60}{\left(\frac{l_{b}}{d}\right)^{3}}+0.0025 \frac{l_{b}}{d}\right] \frac{\left(S_{S}\right)_{e}}{S_{r e f}}\right\} R_{W B}
\end{aligned}
$$

where $\left(C_{D_{o}}\right)_{W B}-C_{D_{b}} \frac{S_{B}}{S_{\text {ref }}}$ represents the wing-body zero-lift drag minus the base drag.

Step 6: The base drag $C_{D_{b}}$ was determined using the following method: 


$$
\begin{gathered}
\left(C_{D_{f}}\right)_{b}=\left(C_{f}\right)_{B}\left\lfloor 1+\frac{60}{\left(\frac{l_{b}}{d}\right)^{3}}+0.0025 \frac{l_{b}}{d}\right\rfloor \frac{\left(S_{S}\right)_{e}}{S_{B}} \\
C_{D_{b}}=0.029 \frac{\left(\frac{d_{b}}{d}\right)^{3}}{\sqrt{\left(C_{D_{f}}\right)_{b}}}
\end{gathered}
$$

where $\left(\frac{d_{b}}{d}\right)$ is a ratio of diameters of the body cross section (blunt base diameter over maximum body diameter) and is described using Figure 91.

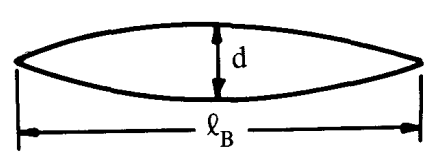

CLOSED BODY

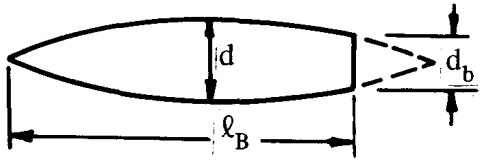

BODY HAVING A BLUNT BASE

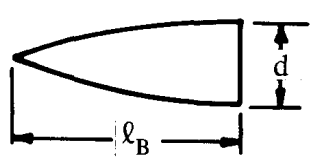

FOREBODY

Figure 91: Body Cross-Section Geometries [Reproduced from Reference41]

Step 7: The total wing-body zero-lift drag was calculated using Equation 7.8:

$$
\left(C_{D_{o}}\right)_{W B}=\left(\left(C_{D_{o}}\right)_{W B}-C_{D_{b}} \frac{S_{B}}{S_{r e f}}\right)+C_{D_{b}} \frac{S_{B}}{S_{r e f}}=0.0057+0.0060\left(\frac{557.28}{1675.70}\right)=0.0077
$$

Step 8: The horizontal and vertical tail zero-lift drag contributions were determined using Equations 7.9 and 7.10:

$$
\begin{aligned}
& \left(C_{D_{o}}\right)_{H}=\left(C_{f}\right)_{H}\left[1+L\left(\frac{t}{c}\right)+100\left(\frac{t}{c}\right)^{4}\right]_{H}\left(R_{L . S .}\right)_{H} \frac{\left\lfloor\left(S_{w e t}\right)_{H}\right\rfloor_{e}}{S_{r e f}} \\
& \left(C_{D_{o}}\right)_{V}=\left(C_{f}\right)_{V}\left[1+L\left(\frac{t}{c}\right)+100\left(\frac{t}{c}\right)^{4}\right]_{V}\left(R_{L . S .}\right)_{V} \frac{\left\lfloor\left(S_{\text {wet }}\right)_{V}\right\rfloor_{e}}{S_{\text {ref }}}
\end{aligned}
$$

Once each of the steps were completed, the total wing-body-tail contribution to $c_{D_{o}}$ was calculated by summing the contributions from the wing-body and tails and was found to be 0.0087 . 


\subsubsection{Lift-Due-to-Angle-of-Attack Derivative: $C_{L_{\alpha}}$}

The value for $C_{L_{\alpha}}$ was determined for the wing-body-tail approximation. DATCOM provides several different methods for the approximation of this coefficient, but for this case, the wing and body lift-curve slope were determined separately and combined. The wing-body approximation from DATCOM depends on slender-body theory, which is not applicable in the case of the WVU YF-22. Still, since there is no method available for estimating the lift-curve slope of a body of non-circular cross sections, this is highly approximated and does not account for the interactions between the wing and body. Typically, one would rely on test data as the basis for making a prediction of the lift-curve slope of a body with a non-circular cross section.

$$
\left(C_{L_{\alpha}}\right)_{W B T}=\left[K_{N}+K_{W(B)}+K_{B(W)}\right]^{\prime}\left(C_{L_{\alpha}}\right)_{e}^{\prime} \frac{S_{e}^{\prime}}{S^{\prime}}+\left[K_{W(B)}+K_{B(W)}\right]^{\prime \prime}\left(C_{L_{\alpha}}\right)_{e}^{\prime \prime}\left(1-\frac{d \varepsilon}{d \alpha}\right) \frac{q^{\prime \prime}}{q_{\infty}} \frac{S_{e}^{\prime \prime}}{S^{\prime \prime}}
$$

First the calculations were completed for the wing contribution to the wing-body-tail liftcurve slope. Figure 92 was used to determine the wing lift-curve slope correction factor from the wing thickness and geometry. The geometric parameter required for this chart must be calculated for just the wing; therefore, the outer wing parameters of the YF-22 were used.

$$
\frac{1}{3+A R}\left(\frac{1-\lambda}{1+2 \lambda}\right)^{2}
$$

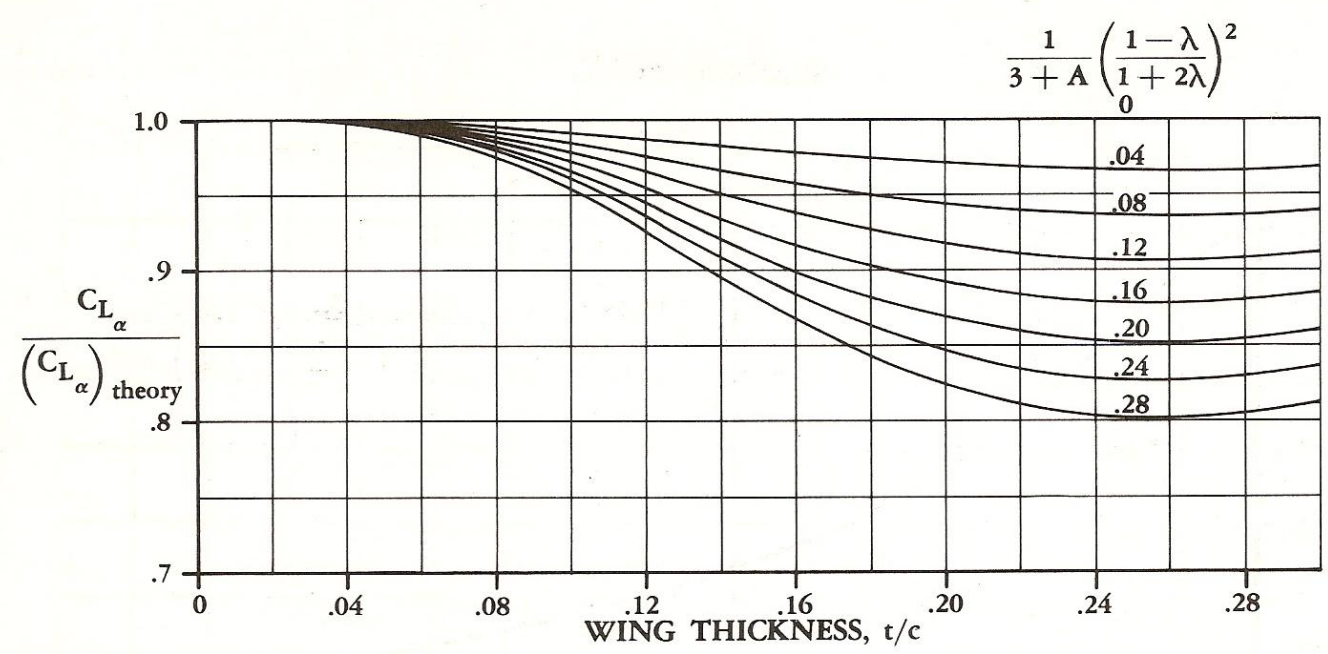

Figure 92: Wing Lift-Curve Slope Correction Factor [Reproduced from Reference 41] 
Using Figure 92 and knowing the wing thickness-to-chord ratio, $t / c$, to be 0.074 , the value for the ratio of the lift-curve slope correction factor was determined. Next, the theoretical lift-curve slope, $\left(C_{L_{\alpha}}\right)_{\text {theory }}$, was calculated using the following equation:

$$
\left(C_{L_{\alpha}}\right)_{\text {theory }}=8 \tan ^{-1} \frac{\pi A R}{16+\pi A R /\left(1+2 \lambda \tan \Lambda_{L E}\right)}
$$

In this case, the aspect ratio of the exposed wing was used, which is considered as the outer wing panel for the WVU YF-22.

The same methodology was used to calculate the lift-curve slope of the exposed horizontal stabilizer - in the case of the WVU YF-22, the stabilators.

Next, the lift ratios $K_{W(B)}$ and $K_{B(W)}$ were calculated for both the wing and horizontal tail using Figure 93.

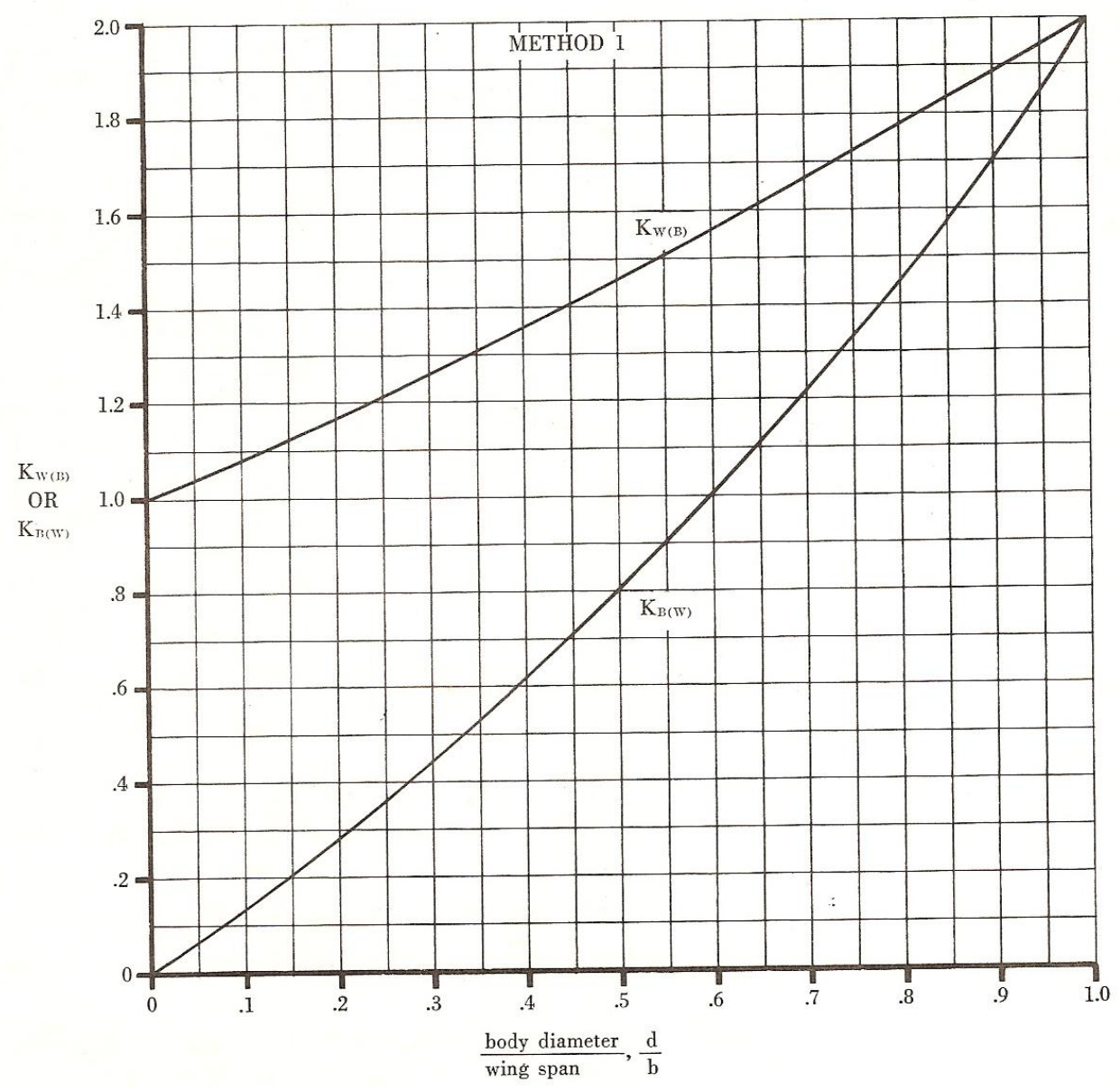

Figure 93: Lift Ratios KW(B) and KB(W) - Slender Body Theory - Fixed Incidence - All Speeds [Reproduced from Reference 41] 
Next, $K_{N}$ was calculated for the wing using Equation 7.14:

$$
K_{N}=\frac{\left(C_{L_{\alpha}}\right)_{N} S_{N_{r e f}}}{\left(C_{L_{\alpha}}\right)_{e} S_{e}}
$$

Applying these values to the lift-curve slope equation for the wing-body-tail approximation, the total value was obtained.

\subsubsection{Pitching-Moment-Due-to-Angle-of-Attack Derivative: $C_{m_{\alpha}}$}

To calculate the wing-body-tail $C_{m_{\alpha}}$ for the WVU YF-22 where $\frac{b^{\prime}}{b^{\prime \prime}} \geq 1.5$, Equation 7.15 was used:

$$
C_{m_{\alpha}}=-\frac{x_{C G}-x^{\prime}}{\bar{c}^{\prime}}\left(C_{L_{\alpha}}\right)^{\prime}-\frac{x_{C G}-x^{\prime \prime}}{\bar{c}^{\prime \prime}}\left(C_{L_{\alpha}}\right)^{\prime \prime} \frac{q^{\prime \prime}}{q_{\infty}} \frac{S^{\prime \prime}}{S^{\prime}} \frac{\bar{c}^{\prime \prime}}{\bar{c}^{\prime}}\left(1-\frac{\partial \varepsilon}{\partial \alpha}\right)
$$

where:

$$
\begin{gathered}
\frac{x_{C G}-x^{\prime}}{\bar{c}^{\prime}}=\frac{x_{C G}}{\bar{c}^{\prime}}+\left(\frac{x_{A C}^{\prime}}{\bar{c}_{r}^{\prime}}\right)\left(\frac{c_{r}^{\prime}}{\bar{c}^{\prime}}\right)-\frac{g^{\prime}}{\bar{c}^{\prime}}-0.25 \\
\frac{x_{C G}-x^{\prime \prime}}{\bar{c}^{\prime \prime}}=\frac{l^{\prime \prime}}{\bar{c}^{\prime \prime}}+\frac{x_{C G}}{\bar{c}^{\prime \prime}}
\end{gathered}
$$

where $\frac{x_{C G}}{\bar{c}^{\prime}}$ is the distance between the leading edge vertex of the wing at the mean aerodynamic chord to the center of gravity of the aircraft divided by the mean aerodynamic chord of the wing. All terms with (,) represent characteristics of the main wing, while terms with (") represent characteristics of the horizontal tail ${ }^{41}$. Figure 94 highlights some of these basic geometric characteristics. To calculate $\left(\frac{x_{A C}^{\prime}}{c_{r}^{\prime}}\right)$, Equation 7.18 was used:

$$
\left(\frac{x_{A C}^{\prime}}{c_{r}^{\prime}}\right)=\frac{\left(\frac{2}{3}\right)(1-\lambda)+\left(\frac{1}{2}\right)\left\lfloor 1-\frac{\lambda^{2}}{(1+\lambda)}\right\rfloor \pi \log _{e}\left(1+\frac{A R}{5}\right)}{1+\pi \log _{e}\left(1+\frac{A R}{5}\right)}
$$




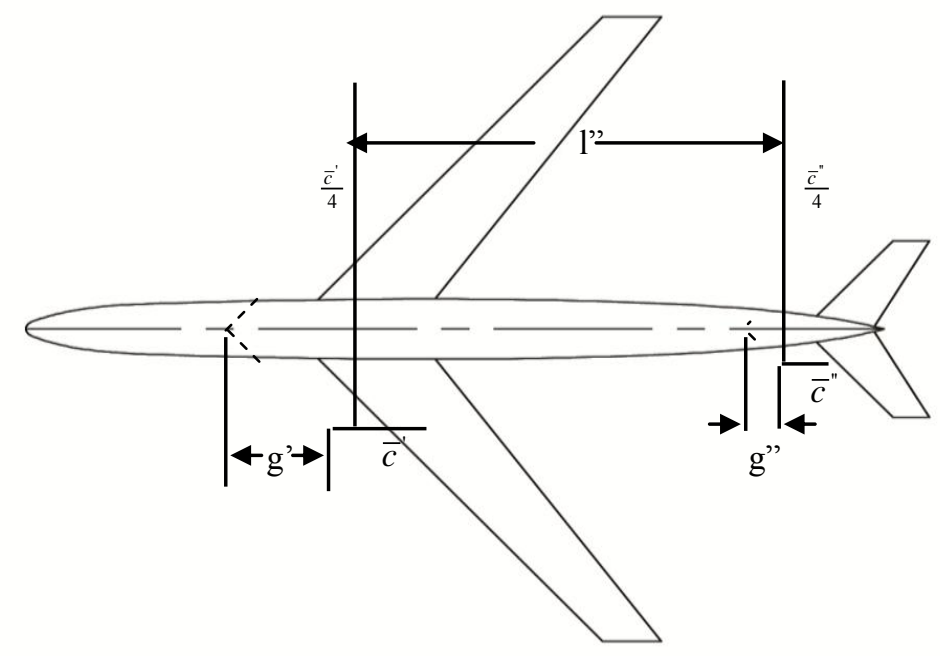

Figure 94: Aircraft Geometric Parameters [Reproduced from Reference 41]

\subsubsection{Pitching-Moment-Due-to-Pitch-Rate Derivative: $c_{m_{q}}$}

The wing-body-tail contribution to $c_{m_{q}}$ was calculated using Equation 7.19:

$$
C_{m_{q}}=\left(C_{m_{q}}\right)_{W B}-2\left[K_{W(B)}+K_{B(W)}\right]^{\prime \prime}\left(\frac{S_{e}^{\prime \prime}}{S^{\prime}}\right)\left(\frac{x_{C G}-x^{\prime \prime}}{\bar{c}^{\prime}}\right)^{2}\left(\frac{q^{\prime \prime}}{q_{\infty}}\right)\left(\frac{d \varepsilon}{d \alpha}\right)\left(C_{L_{\alpha}}\right)_{e}^{\prime \prime}
$$

Equation 7.20 was used to determine $\left(C_{m_{q}}\right)_{W B}$ for the WVU YF-22:

$$
\left(C_{m_{q}}\right)_{W B}=\left[K_{W(B)}+K_{B(W)}\right]\left(\frac{S_{e}}{S}\right)\left(\frac{\bar{c}_{e}}{\bar{c}}\right)^{2}\left(C_{m_{q}}\right)_{e}+\left(C_{m_{q}}\right)_{B}\left(\frac{S_{b}}{S}\right)\left(\frac{l_{B}}{\bar{c}}\right)^{2}
$$

For the effective wing pitching derivative, the calculations were assumed for a Mach number of approximately 0.2 , for lack of more appropriate options for this aircraft.

$$
\left(C_{m_{q}}\right)_{M \approx 0.2}=-0.7 c_{l_{\alpha}} \cos \Lambda_{c / 4}\left\{\frac{A R\left[\frac{1}{2} \frac{\bar{x}}{\bar{c}}+2\left(\frac{\bar{x}}{\bar{c}}\right)^{2}\right]}{A R+2 \cos \Lambda_{c / 4}}+\frac{1}{24}\left(\frac{A R^{3} \tan ^{2} \Lambda_{c / 4}}{A R+6 \cos \Lambda_{c / 4}}\right)+\frac{1}{8}\right\} \text { (per rad) }
$$

The body pitching derivative was obtained using Equation 7.22: 


$$
\left(C_{m_{q}}\right)_{B}=2 C_{m_{\alpha}} \frac{\left[\left(1-\frac{x_{m}}{l_{B}}\right)^{2}-\frac{V_{B}}{S_{b} l_{B}}\left(\frac{x_{c}}{l_{B}}-\frac{x_{m}}{l_{B}}\right)\right]}{\left[\left(1-\frac{x_{m}}{l_{B}}\right)-\frac{V_{B}}{S_{b} l_{B}}\right]}
$$

The variables used in Equation 7.22 are illustrated in Figure 95. The variables are defined as follows: $l_{b}$ is the length of the aircraft body, $l_{N}$ is the length of the nose, $S_{b}$ is the side area of the aircraft body, and $x_{m}$ is the longitudinal distance from the body vertex to the center of rotation (positive aft).

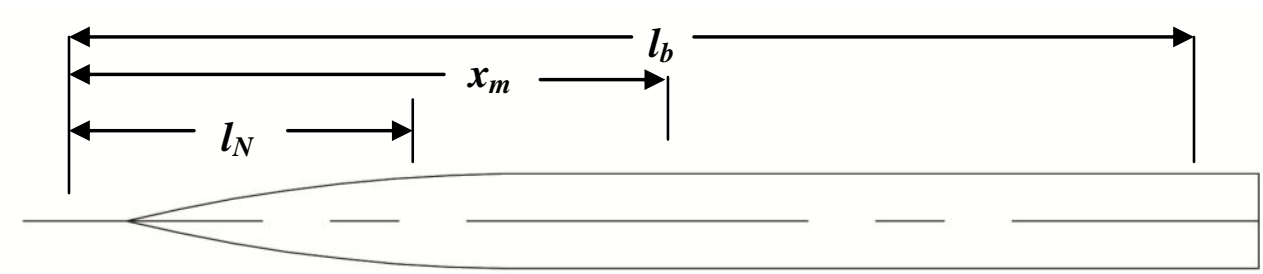

Figure 95: Fuselage Longitudinal Geometric Parameters [Reproduced from Reference 41]

Applying the effective and body pitching derivatives to the wing-body equation allowed for the wing-body pitching derivative to be calculated. The reader should be aware that one limitation of this method for calculating $\left(C_{m_{q}}\right)_{W B}$ is determined by the limitations of the methods employed in determining the static derivatives $C_{m_{\alpha}}$ in the various speed ranges. Once $\left(C_{m_{q}}\right)_{W B}$ was calculated, it was applied back into the equation for the wing-body-tail pitching derivative $C_{m_{q}}$.

\subsubsection{Rolling-Moment-Due-to-Sideslip Derivative:}

The wing-body-tail $c_{l_{\beta}}$ was calculated using Equation 7.23.

$$
C_{l_{\beta}}=\left(C_{l_{\beta}}\right)_{W B}+\Sigma_{p}\left(\left(\Delta C_{Y_{\beta}}\right)_{p}\left(\frac{z_{p} \cos \alpha-l_{p} \sin \alpha}{b_{W}}\right)\right)
$$

The case of an aircraft flying in subsonic conditions with straight-tapered wings, sideslip angles between positive or negative 50 degrees, and at low angles of attack was considered for this DATCOM-based calculation. 


$$
\begin{aligned}
\left(C_{l_{\beta}}\right)=C_{L} & \left\lfloor\left(\frac{C_{l_{\beta}}}{C_{L}}\right)_{\Lambda_{c / 2}} K_{M_{\Lambda}} K_{f}+\left(\frac{C_{l_{\beta}}}{C_{L}}\right)_{A R}\right\rfloor+\Gamma\left[\frac{C_{l_{\beta}}}{\Gamma} K_{M_{\Gamma}}+\frac{\Delta C_{l_{\beta}}}{\Gamma}\right]+\left(\Delta C_{l_{\beta}}\right)_{z_{W}} \text { (per deg) } \\
& +\theta \tan \Lambda_{c / 4}\left(\frac{\Delta C_{l_{\beta}}}{\theta \tan \Lambda_{c / 4}}\right)
\end{aligned}
$$

Since the WVU YF-22 has no wing twist between the root and tip sections, $\theta$ was set equal to zero. Also, the wings are at a zero degree dihedral, so $\Gamma$ was set equal to zero. Using the exposed wing, the following method was followed to obtain the other parameters required in Equation 7.24:

The wing sweep contribution $\left(\frac{C_{l_{\beta}}}{C_{L}}\right)_{\Lambda_{c / 2}}$ was found from Figure 96 for a tip ratio of 0.5. 

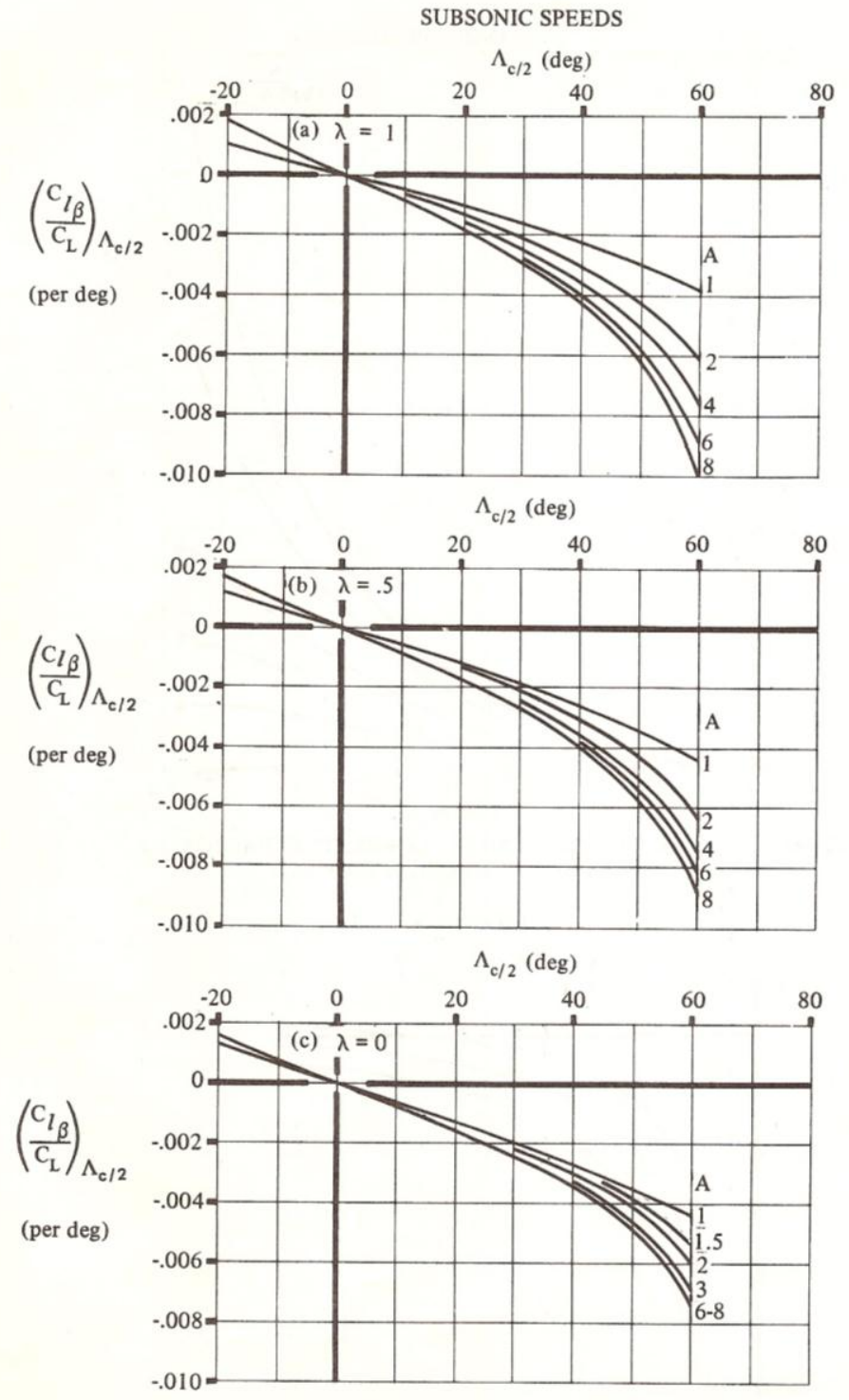

FIGURE 5.1.2.1-27 WING SWEEP CONTRIBUTION TO C $l_{\beta}$

Figure 96: Wing Sweep Contribution to $C_{l_{\beta}}$ [Reproduced from Reference 41]

The compressibility correction to the sweep contribution, $K_{M_{\wedge}}$, was found from Figure 97 based on the following calculations:

$$
\begin{gathered}
\frac{A R}{\cos \left(\Lambda_{c / 2}\right)}=\frac{2.19}{\cos \left(10^{\circ}\right)}=2.23 \\
M \cos \left(\Lambda_{c / 2}\right)=0.123 \cos \left(10^{\circ}\right)=0.122
\end{gathered}
$$




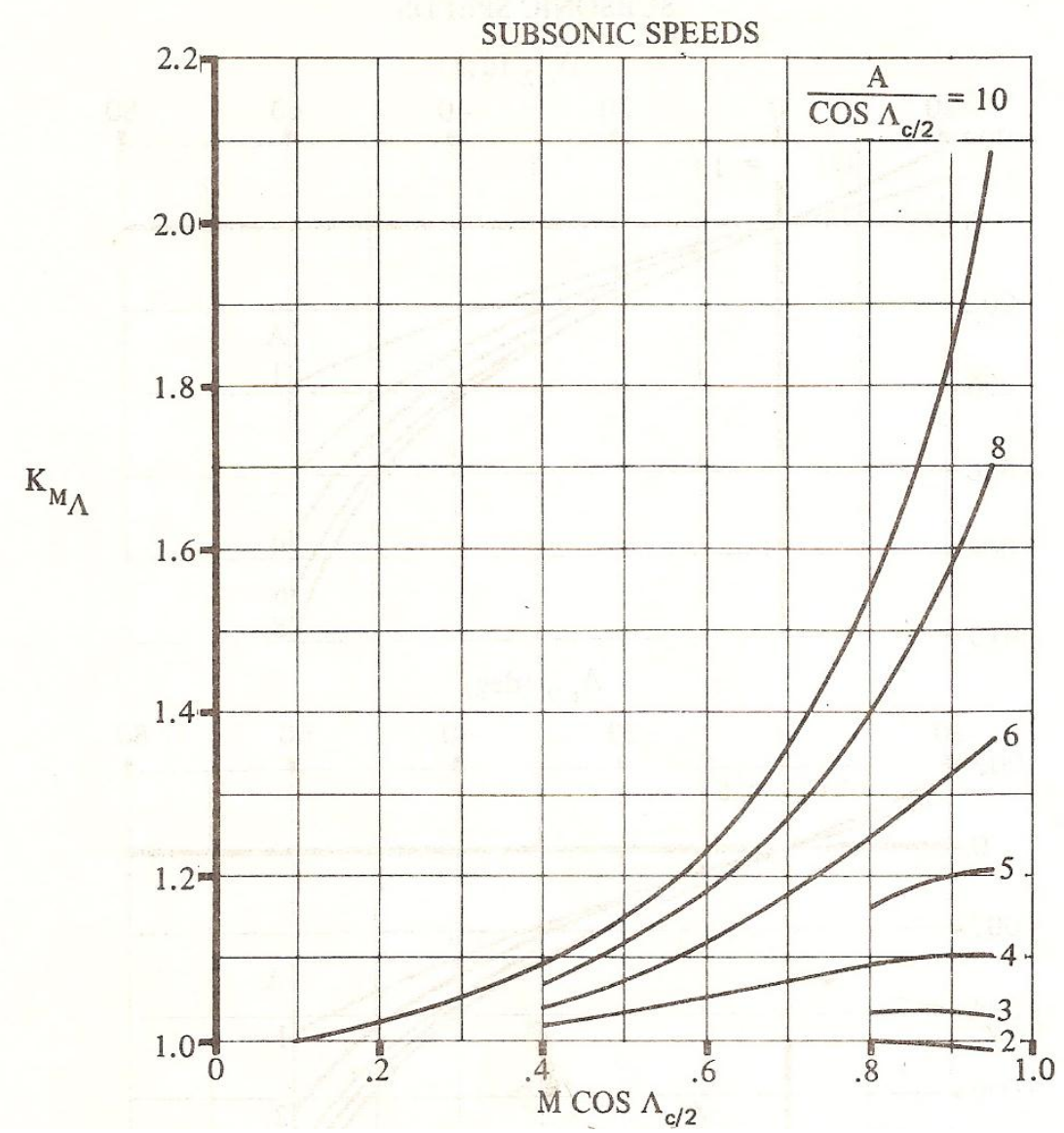

Figure 97: Compressibility Correction Factor to Sweep Contribution to Wing $C_{l_{\beta}}$ [Reproduced from Reference 41]

The empirical fuselage-length-effect correction factor, $K_{f}$, was found from Figure 98, the aspect ratio contribution $\left(\frac{C_{l_{\beta}}}{C_{L}}\right)_{A R}$ was found using Figure 99, and the increment in $C_{l_{\beta}}$ due to the body-induced effect on wing height for configurations with wings located above or below the mid-fuselage height $\left(\Delta C_{l_{\beta}}\right)_{z_{W}}$ was calculated by Equation 7.27:

$$
\left(\Delta C_{l_{\beta}}\right)_{z_{W}}=\frac{1.2 \sqrt{A R}}{57.3}\left(\frac{z_{W}}{b}\right)\left(\frac{2 d}{b}\right)(\text { per deg })
$$




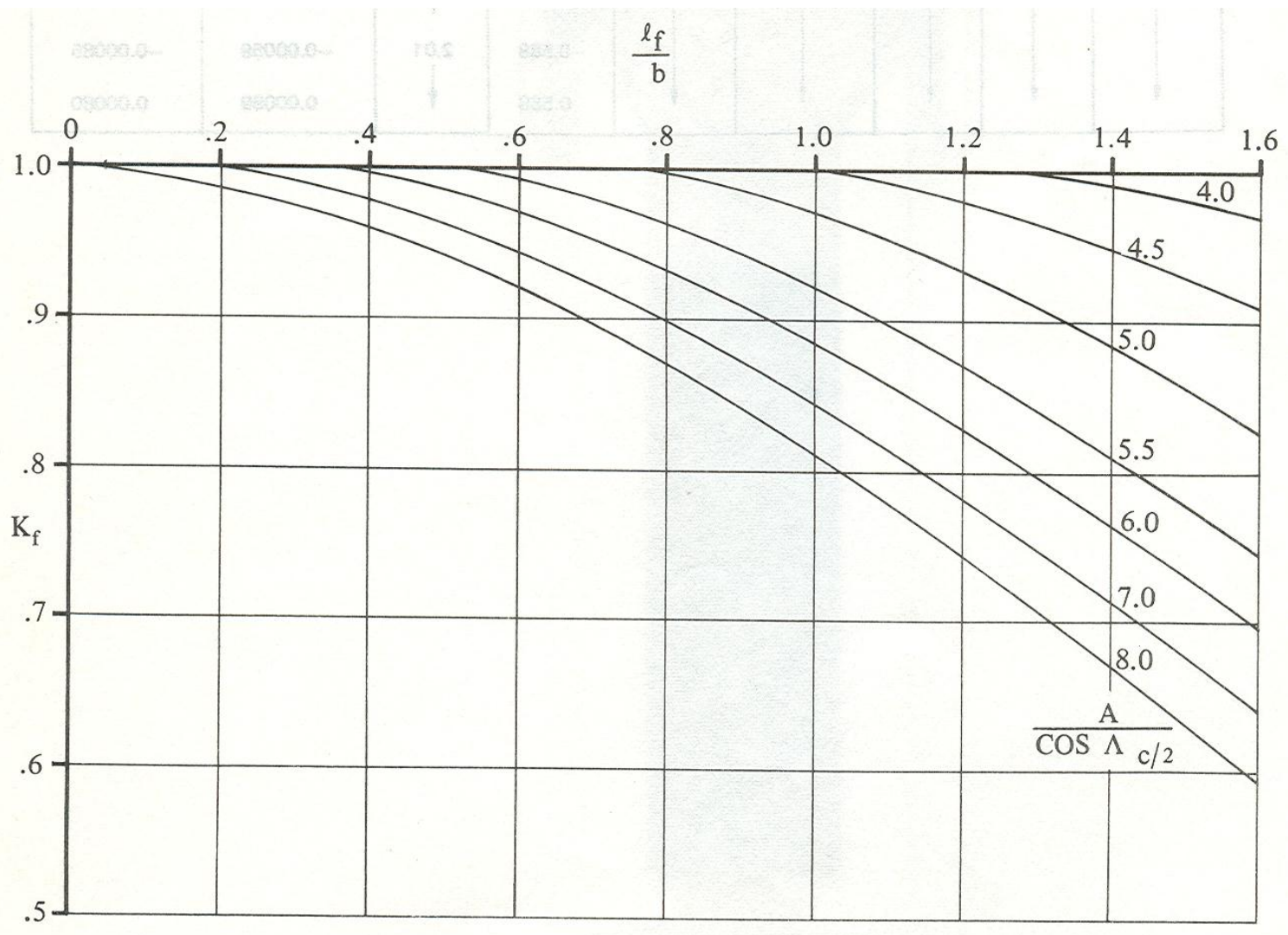

Figure 98: Fuselage Correction Factor [Reproduced from Reference 41]

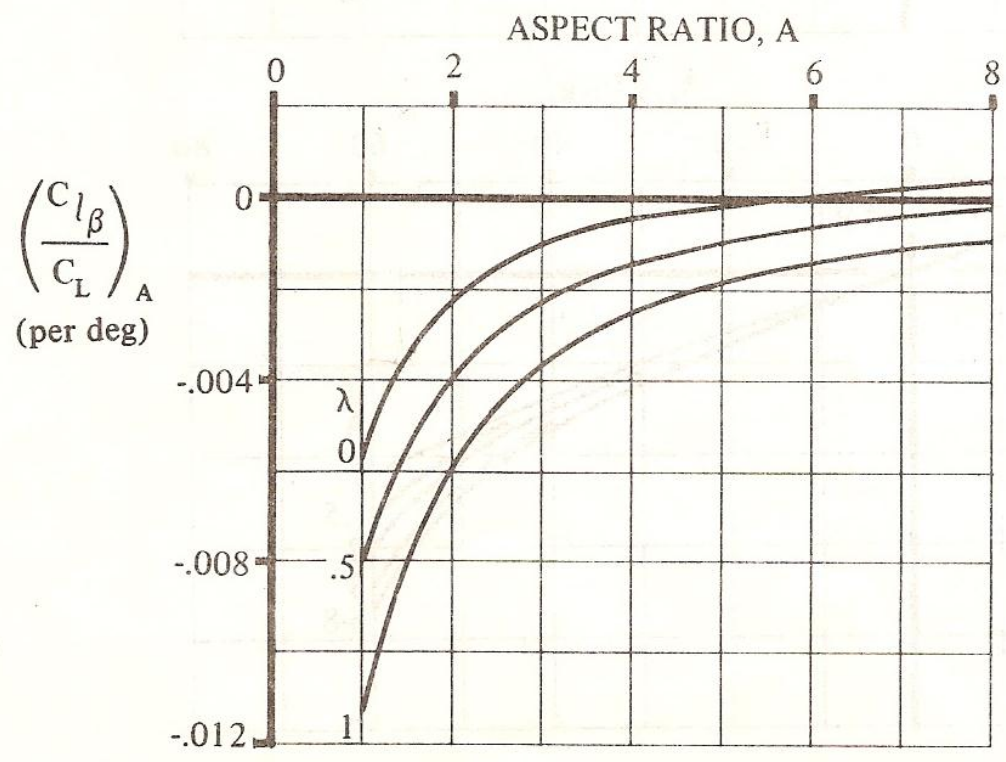

Figure 99: Aspect Ratio Contribution to Wing $C_{l_{\beta}}$ [Reproduced from Reference 41]

Using these values, the wing-body contribution to $c_{l_{\beta}}$ was calculated using Equation 7.28. 


$$
\left(C_{l_{\beta}}\right)=C_{L}\left\lfloor\left(\frac{C_{l_{\beta}}}{C_{L}}\right)_{\Lambda_{c / 2}} K_{M_{\Lambda}} K_{f}+\left(\frac{C_{l_{\beta}}}{C_{L}}\right)_{A R}\right\rfloor+\left(\Delta C_{l_{\beta}}\right)_{z_{W}}
$$

$\left(\Delta C_{Y_{\beta}}\right)_{p}$ was calculated using Equation 7.29 for an aircraft configuration with twin vertical panels mounted on the tips of the horizontal tail:

$$
\left(C_{l_{\beta}}\right)_{p}=\left(\Delta C_{Y_{\beta}}\right)_{V(W B H)}\left[\frac{z_{V} \cos \alpha-l_{v} \sin \alpha}{b_{W}}\right]
$$

This calculation for $\left(\Delta C_{Y_{\beta}}\right)_{V(W B H)}$ must be done for varying angles of attack, $\alpha$, but in this case it was considered at $3.0^{\circ}$ using Equation 7.30.

$$
\left(\Delta C_{Y_{\beta}}\right)_{V(W B H)}=-\frac{\left(C_{Y_{\beta}}\right)_{V(W B H)}}{\left(C_{Y_{\beta}}\right)_{V_{\text {eff }}}}\left(C_{Y_{\beta}}\right)_{V_{\text {eff }}} \frac{2 S_{V}}{S_{W}}
$$

This twin vertical tail method was used in the approximation despite the fact that the vertical tails are mounted on the rear of the fuselage. This method provided the closest estimation in the case of the WVU YF-22. Table 40 provides the geometric parameters of the WVU YF-22 necessary for these calculations.

Table 40: Aircraft Geometric Parameters for Calculating $\left(\Delta C_{Y_{\beta}}\right)_{V(W B H)}$

\begin{tabular}{|c|c|c|}
\hline Dimension & Value & Units \\
\hline $\boldsymbol{b}_{\boldsymbol{v}}$ & 17 & in \\
\hline $\boldsymbol{b}_{\boldsymbol{v}}$ & 14 & in \\
\hline $\boldsymbol{b}_{\boldsymbol{w}}$ & 72.49 & in \\
\hline $\boldsymbol{b}_{\boldsymbol{H}}$ & 15 & in \\
\hline $\boldsymbol{I}_{\boldsymbol{p}}$ & 21.0 & in \\
\hline $\boldsymbol{z}_{\boldsymbol{p}}$ & 6.0 & in \\
\hline $\boldsymbol{r}_{\boldsymbol{1}}$ & 3.0 & in \\
\hline $\boldsymbol{S}_{\boldsymbol{v}}$ & 180.14 & in $^{2}$ \\
\hline
\end{tabular}

The first step to calculate $\left(\Delta C_{Y_{\beta}}\right)_{V(W B H)}$ was to calculate $\frac{A_{e f f}}{A}$ from Figure 100 as a function of the geometric variables shown. Once this was calculated, the lift-curve slope of the equivalent rectangular vertical panel $\left(C_{Y_{\beta}}\right)_{V_{e f f}}$ and $\frac{\left(C_{Y_{\beta}}\right)_{V(W B H)}}{\left(C_{Y_{\beta}}\right)_{V_{e f f}}}$ was obtained. 


$$
\begin{aligned}
& \frac{A_{e f f}}{A}=1.23 \\
& \left(C_{Y_{\beta}}\right)_{V_{e f f}}=1.8 \text { per radian } \\
& \frac{\left(C_{Y_{\beta}}\right)_{V(W B H)}}{\left(C_{Y_{\beta}}\right)_{V_{e f f}}}=0.71 \text { per radian }
\end{aligned}
$$

The total wing-body-tail contribution to $c_{l_{\beta}}$ was calculated to as follows using the different contributions. 
SUBSONIC
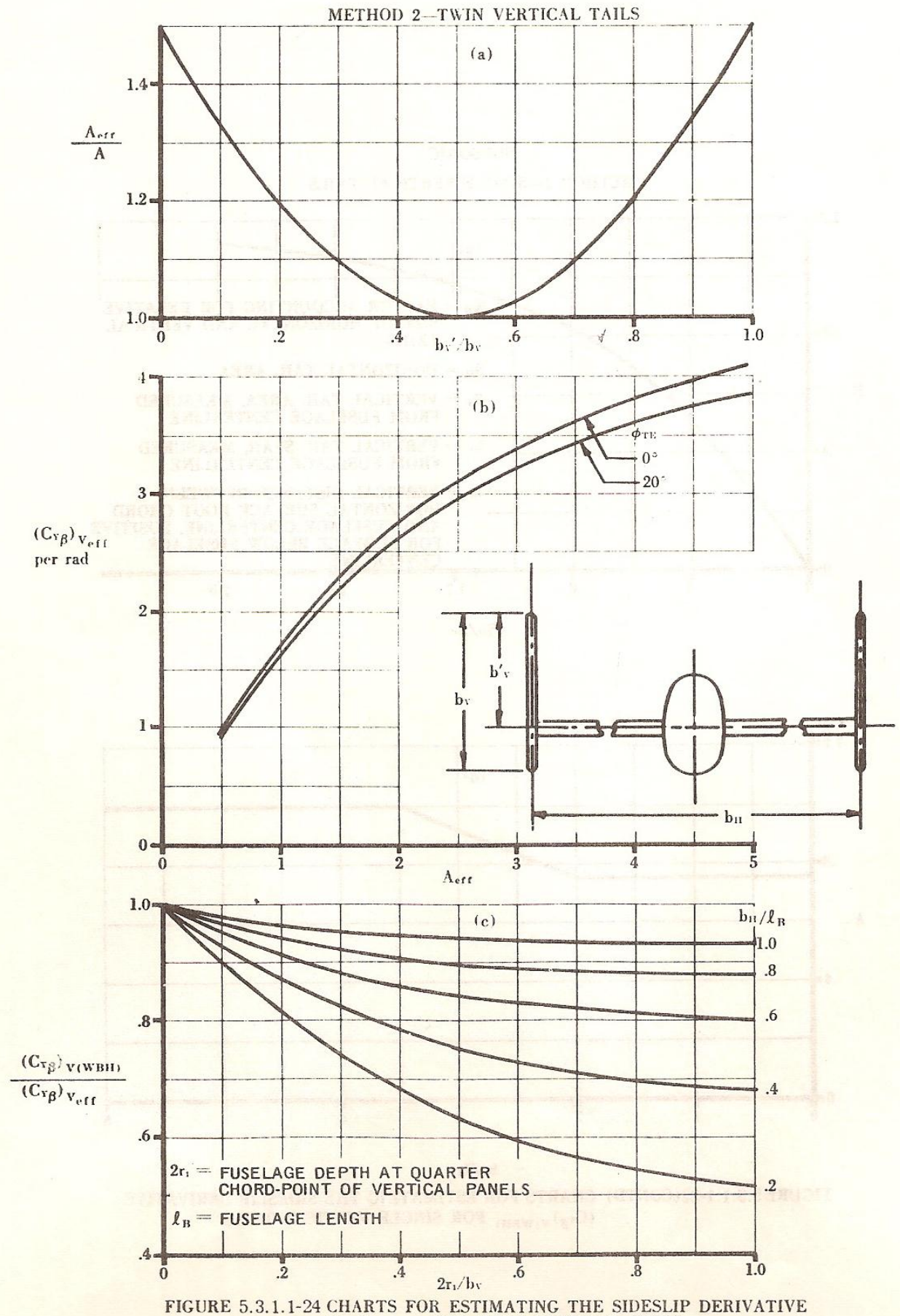

FIGURE 5.3.1.1-24 CHARTS FOR ESTIMATING THE SIDESLIP DERIVATIVE $\left.\left(C_{Y_{B}}\right)_{V(W B H}\right)$ FOR TWIN VERTICAL TAILS.

Figure 100: Charts for Estimating the Sideslip Derivative $\left(\Delta C_{Y_{\beta}}\right)_{V(W B H)}$ for Twin Vertical Tails [Reproduced from Reference 41] 


\subsubsection{Rolling Moment-Due-to-Roll-Rate Derivative: $C_{l_{p}}$}

The wing contribution to the rolling derivative $C_{l_{p}}$ was calculated first. The wing-body assumption is invalid for this case because $d / b$ is greater than 0.3 , so the wing rolling derivative alone was calculated. The airfoil of the wing was required for this calculation; however, the WVU YF-22 has a varying profile along the wing, namely, four different airfoils. The root airfoil was used to determine the main wing characteristics. The root airfoil for the aircraft is the NACA 1408, so using the basic properties of the airfoil the lift-curve slope of the wing was approximated for this application.

For the NACA 1408 on the WVU YF-22, the lift-curve slope of the wing is 6.18 (per radian). Using the following formula, the wing-body contribution to the rolling derivative was calculated for the WVY YF-22:

$$
C_{l_{p}}=\left(\frac{\beta C_{l_{p}}}{\kappa}\right)_{C_{L}=0}\left(\frac{\kappa}{\beta}\right) \frac{\left(C_{L_{\alpha}}\right)_{C_{L}}}{\left(C_{L_{\alpha}}\right)_{C_{L}=0}} \frac{\left(C_{l_{p}}\right)_{\Gamma}}{\left(C_{l_{p}}\right)_{\Gamma=0}}+\left(\Delta C_{l_{p}}\right)_{d r a g} \quad \text { (per rad) }
$$

Where $\left(\frac{\beta C_{l_{p}}}{\kappa}\right)_{C_{L}=0}$ is the roll damping parameter at zero lift, $\left(C_{L_{\alpha}}\right)_{C_{L}=0}$ is the wing lift-curve slope at zero lift, $\left(C_{L_{\alpha}}\right)_{C_{L}}$ is the wing lift-curve slope at any lift coefficient below stall, $\frac{\left(C_{l_{p}}\right)_{\Gamma}}{\left(C_{l_{p}}\right)_{\Gamma=0}}$ is the dihedral effect parameter, and $\left(\Delta C_{l_{p}}\right)_{d r a g}$ is the increment in the roll-damping derivative due to drag. Each of these parameters was obtained via the procedure described in the following sections.

To determine $\left(\frac{\beta C_{l_{p}}}{\kappa}\right)_{C_{L}=0}, \beta$ and the ratio of the two-dimensional lift-curve slope at the Mach number to $\frac{2 \pi}{\beta}, \kappa$, was calculated. Equations 7.32 and 7.33 show the determination of the required components $\beta$ and $\kappa$. 


$$
\begin{gathered}
\beta=\sqrt{1-M^{2}}=\sqrt{1-0.123^{2}}=0.992 \\
\kappa=\frac{\left(C_{L_{\alpha}}\right)_{M}}{\left(\frac{2 \pi}{\beta}\right)}=\frac{6.18}{\left(\frac{2 \pi}{0.992}\right)}=0.976
\end{gathered}
$$

Next, the compressible sweep parameter was calculated using Equation 7.34 so that Figure 101 was used to determine $\left(\frac{\beta C_{l_{p}}}{\kappa}\right)_{C_{L}=0}$.

$$
\Lambda_{\beta}=\tan ^{-1}\left(\frac{\tan \Lambda_{c / 4}}{\beta}\right)=\tan ^{-1}\left(\frac{0.38 \mathrm{rad}}{0.992}\right)=0.39 \mathrm{rad}=22.15^{\circ}
$$

(b) $\lambda=0.25$

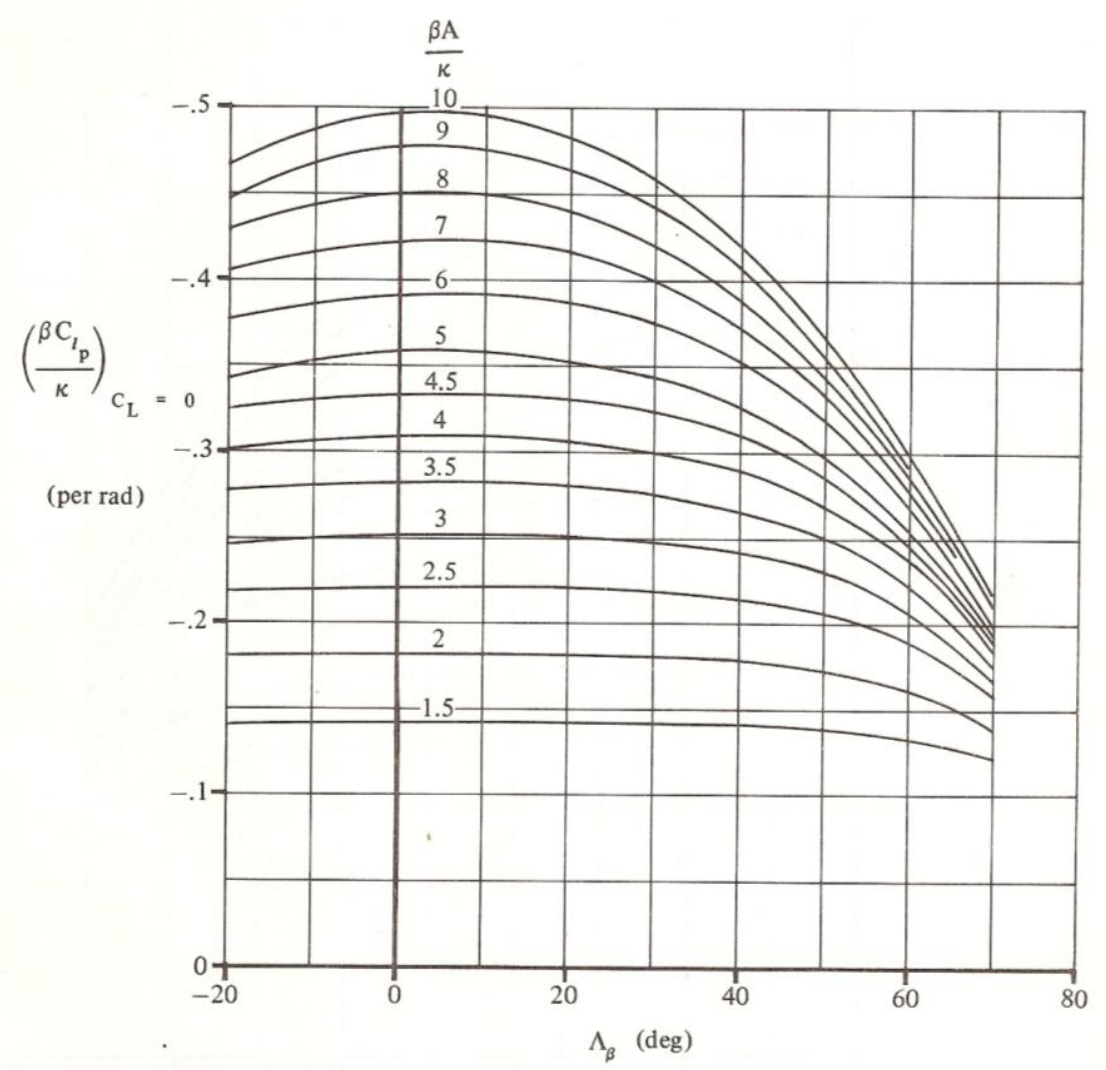

Figure 101: Roll-Damping Parameter at Zero Lift $(\lambda=0.25)$ [Reproduced from Reference 41]

The dihedral effect parameter is 1.0 because the wings are not at a dihedral on the WVU YF-22 aircraft. The increment in the roll-damping derivative due to drag was calculated using Equation 7.35 . 


$$
\left(\Delta C_{l_{p}}\right)_{d r a g}=\frac{\left(C_{l_{p}}\right)_{C_{D_{L}}}}{C_{L}^{2}} C_{L}^{2}-\frac{1}{8} C_{D_{o}}
$$

From Figure 102, the drag-due-to-lift roll-damping parameter $\frac{\left(C_{l_{p}}\right)_{C_{D_{L}}}}{C_{L}^{2}}$ was determined using the aspect ratio of the wings and the quarter-chord sweep angle.

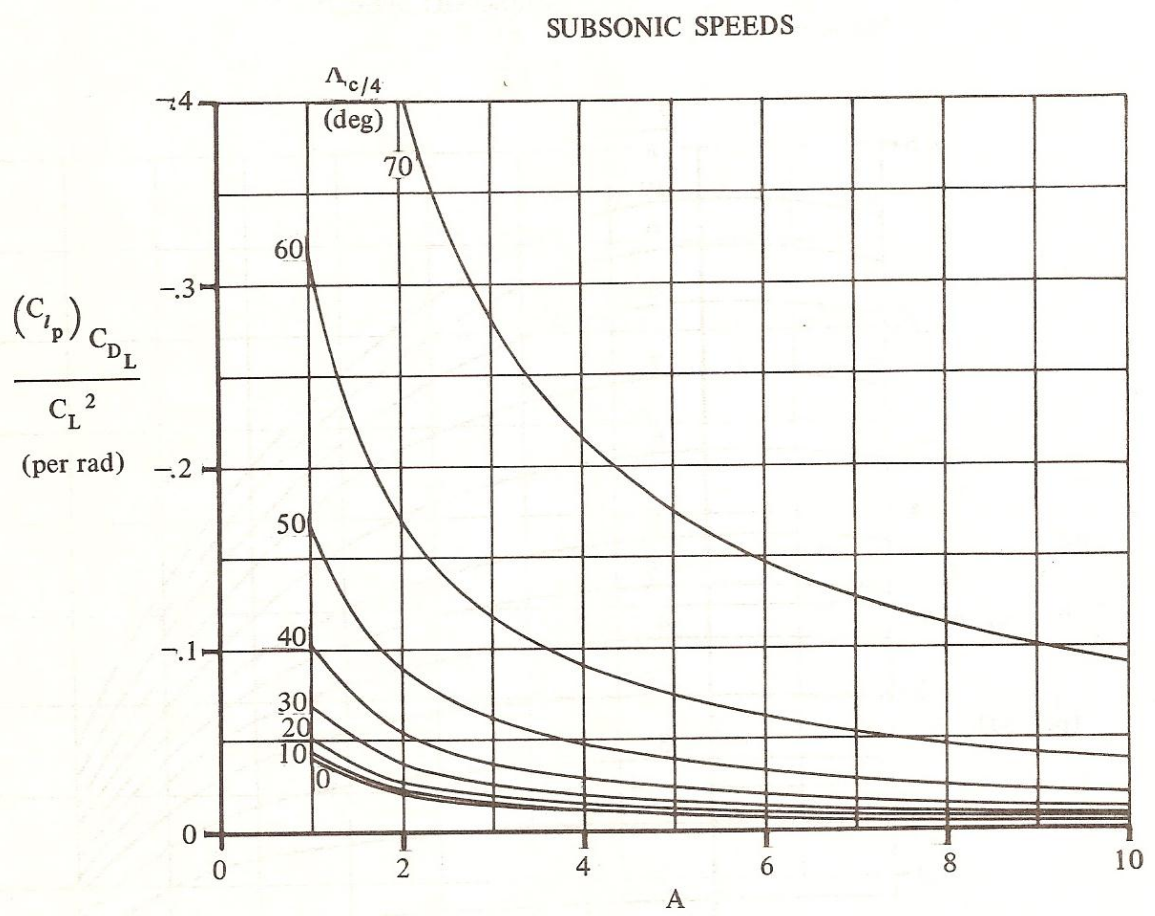

Figure 102: Drag-Due-To-Lift Roll-Damping Parameter [Reproduced from Reference 41]

Using the parameters determined for the WVU YF-22 wings, $\left(\Delta C_{l_{p}}\right)_{d r a g}$ was calculated from Table 41 and found to be -0.0099 . 
Table 41: Step-by-Step Procedure to Determine $\left(\Delta C_{l_{p}}\right)_{d r a g}$ using DATCOM [Reproduced from Reference

\begin{tabular}{|c|c|c|c|}
\hline $\mathbf{( 1 )}$ & $\mathbf{( 2 )}$ & $\mathbf{( 3 )}$ & $\mathbf{( 4 )}$ \\
\hline & & & $\Delta C_{l_{p}}$ \\
$C_{L}$ & $C_{L}^{2}$ & $\frac{1}{8} C_{D_{o}}$ & $-0.034(2)-(3)$ \\
\hline 0 & 0 & 0.0045 & -0.0045 \\
\hline 0.1 & 0.01 & 0.0045 & -0.0048 \\
\hline 0.2 & 0.04 & 0.0045 & -0.0059 \\
\hline 0.3 & 0.09 & 0.0045 & -0.0076 \\
\hline $\mathbf{0 . 4}$ & $\mathbf{0 . 1 6}$ & $\mathbf{0 . 0 0 4 5}$ & $\mathbf{- 0 . 0 0 9 9}$ \\
\hline 0.5 & 0.25 & 0.0045 & -0.0130 \\
\hline 0.6 & 0.36 & 0.0045 & -0.0167 \\
\hline 0.7 & 0.49 & 0.0045 & -0.0212 \\
\hline 0.8 & 0.64 & 0.0045 & -0.0263 \\
\hline
\end{tabular}

From these parameters the wing-rolling derivative was calculated. Next, the same method of calculation was followed for the horizontal tail contribution. For the WVU YF-22, the horizontal tail airfoil is a NACA 0008. $\left(\Delta C_{Y_{\beta}}\right)_{V(W B H)}$ was then calculated using Equation 7.30, and the value of $z$ was calculated using Equation 7.36.

$$
z=z_{p} \cos \alpha-l_{p} \sin \alpha=7.5 \cos \left(3.0^{\circ}\right)-21 \sin \left(3.0^{\circ}\right)=6.39
$$

Applying these values to the equation for the wing-body-tail contribution, the total rolling moment-due-to-roll-rate derivative was calculated using Equation 7.37.

$$
C_{l_{p}}=\left(C_{l_{p}}\right)_{W B}+0.5\left(C_{l_{p}}\right)_{H}\left(\frac{S_{H}}{S_{W}}\right)\left(\frac{b_{H}}{b_{W}}\right)^{2}+\left|2\left(\frac{z}{b_{W}}\right)\left(\frac{z-z_{p}}{b_{W}}\right)\right|\left(\Delta C_{Y_{\beta}}\right)_{V(W B H)}
$$

\subsubsection{Yawing-Moment-Due-to-Sideslip Derivative: $C_{n_{\beta}}$}

The wing-body yawing moment due to sideslip is considered as the sum of the yawing moments of the body and wing-body interference because the wing contribution is not significant except at large incidences. The total wing-body sideslip derivative $C_{n_{\beta}}$ is calculated based on the following equation:

$$
\left(C_{n_{\beta}}\right)_{W B}=-K_{N} K_{R_{l}} \frac{S_{B_{S}}}{S_{W}} \frac{l_{B}}{b} \quad \text { (per deg) }
$$

Using Figure 103 and the aircraft diagram, the necessary measurements were applied to find $K_{N}$. The directional arrows in Figure 103 must be followed to correctly identify $K_{N}$, which was found 
to be 0.001 . The Reynolds number of the air flowing over the aircraft body was multiplied by the length of the body to obtain the empirical Reynolds number factor of $2.1 \times 10^{7} . K_{R_{l}}$, was found to be 1.62 to was with Figure 104.

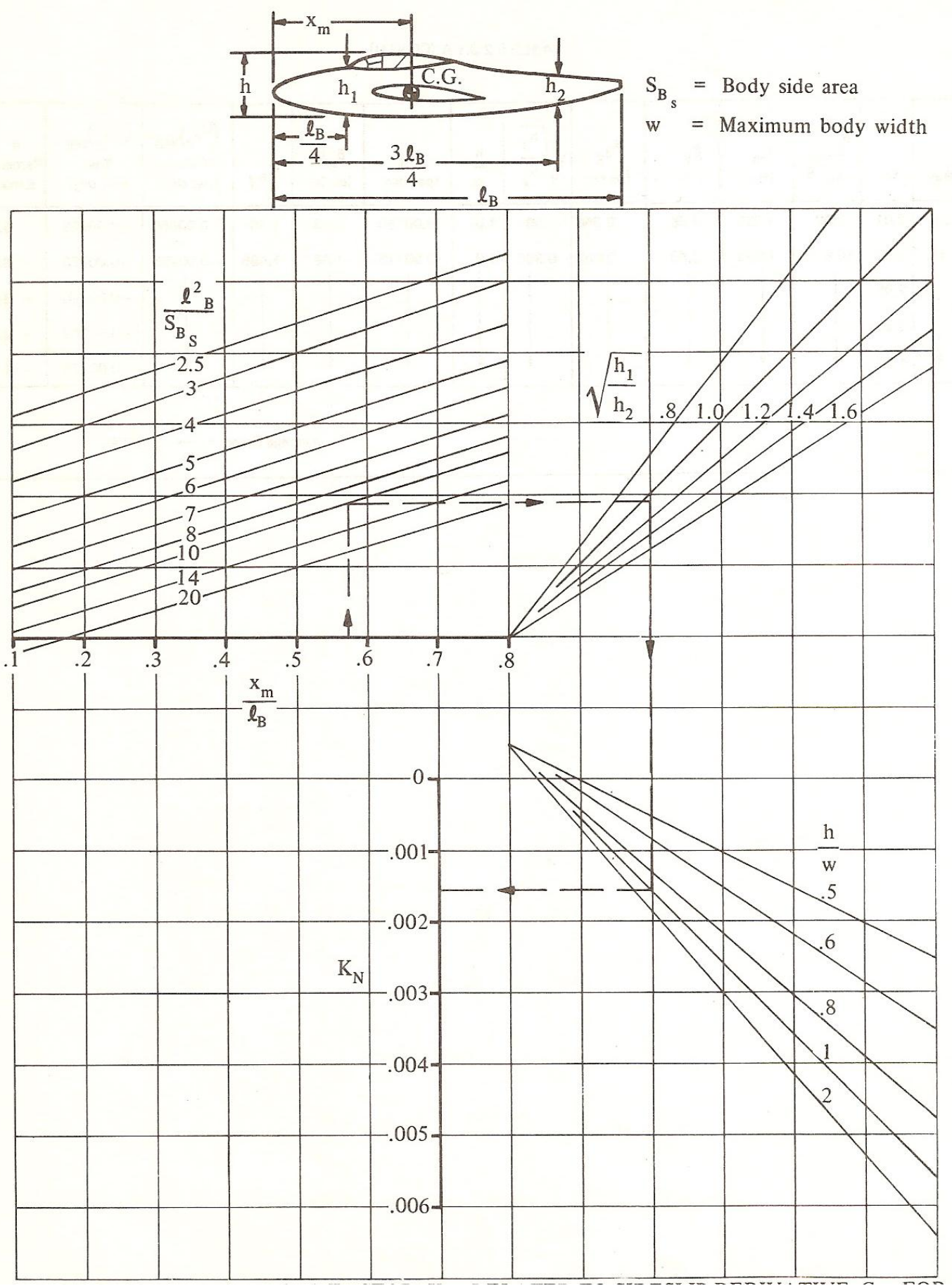

Figure 103: Empirical Factor $\mathrm{K}_{\mathrm{N}}$ Related to Sideslip Derivative $C_{n_{\beta}}$ for Body + Wing-Body Interference

[Reproduced from Reference 41] 


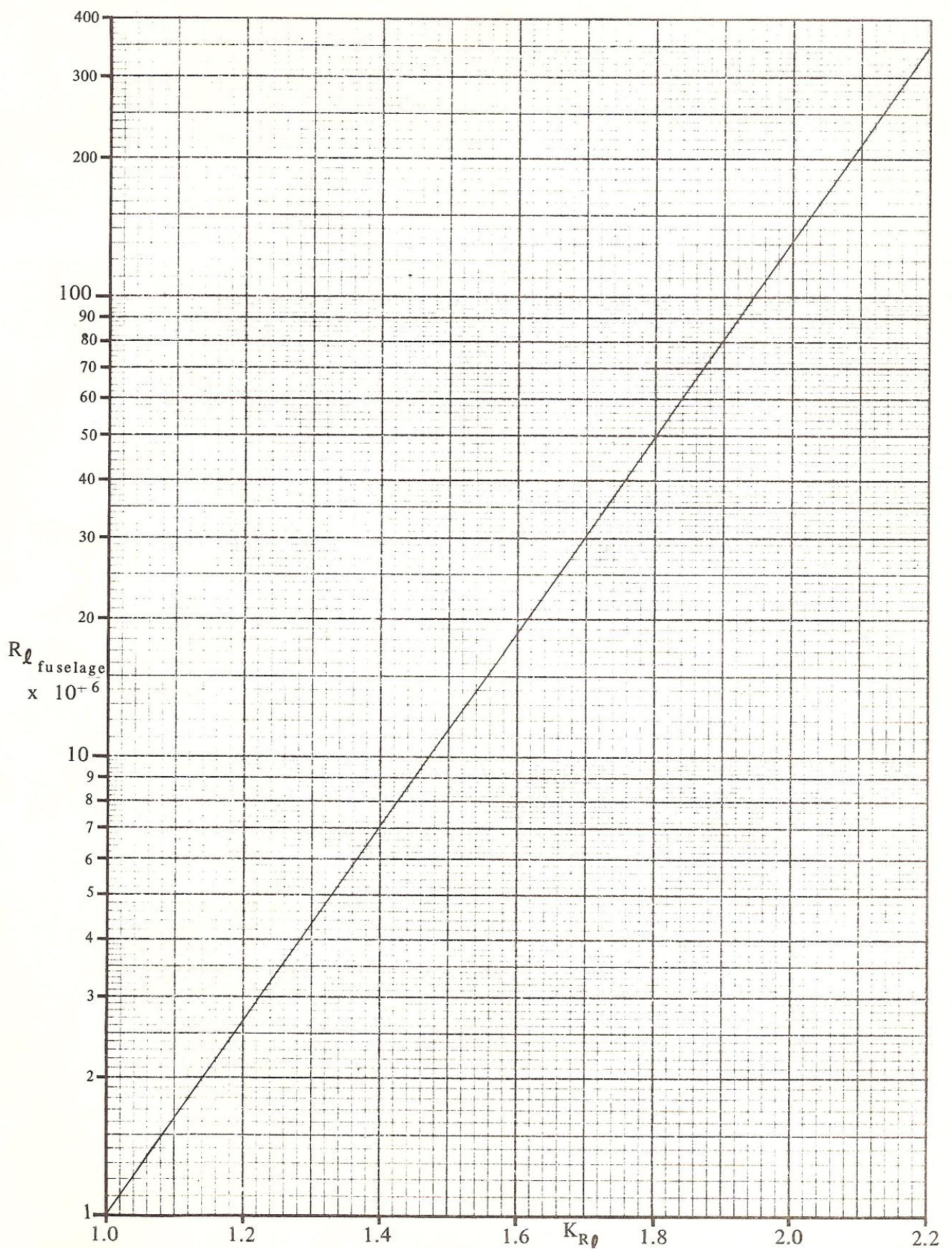

Figure 104: Effect of Fuselage Reynolds number on Wing-Body $C_{n_{\beta}}$ [Reproduced from Reference 41]

Using Equation 7.39, $C_{n_{\beta}}$ was calculated for the wing-body approximation. 


$$
\left(C_{n_{\beta}}\right)_{W B}=-K_{N} K_{R_{l}} \frac{S_{B_{S}}}{S_{W}} \frac{l_{B}}{b}
$$

The vertical tail contribution was calculated and added to the wing-body component using the following equation:

$$
C_{n_{\beta}}=\left(C_{n_{\beta}}\right)_{W B}+\Sigma_{p}\left\lfloor-\left(\Delta C_{Y_{\beta}}\right)_{p} \frac{l_{z}}{b_{w}}\right\rfloor
$$

where $\left(\Delta C_{Y_{\beta}}\right)_{p}$ is the side force due to sideslip of the added panels (the two vertical stabilizers in the case of the WVU YF-22). It can also be considered to be $\left(\Delta C_{Y_{\beta}}\right)_{V(W B H)}$, which is used to find the contribution of twin vertical panels mounted on tips of a horizontal tail. $\left(\Delta C_{Y_{\beta}}\right)_{V(W B H)}$ is the tail-body sideslip derivative, as calculated in Equation 7.30.

\subsubsection{Yawing Moment-Due-to-Yaw-Rate Derivative: $C_{n_{r}}$}

The wing-body-tail contribution to $c_{n_{r}}$ was calculated using the following equation:

$$
C_{n_{r}}=\left(C_{n_{r}}\right)_{W B}+\frac{2}{b_{W}^{2}}\left(l_{p} \cos \alpha+z_{p} \sin \alpha\right)^{2}\left(\Delta C_{Y_{\beta}}\right)_{V(W B H)} \text { (per radian) }
$$

where $\left(C_{n_{r}}\right)_{W B}$ is the wing-body contribution calculated previously, $b_{W}$ is the wing span, $l_{p}$ is the distance parallel the aircraft body $\mathrm{x}$-axis from the moment reference center to the center-ofpressure location of the vertical stabilizer, $z_{p}$ is the distance parallel to the aircraft body $z$-axis from the moment reference center to the center of pressure of the vertical stabilizer (positive for the stabilizer above the body), and $\left(\Delta C_{Y_{\beta}}\right)_{V(W B H)}$ is the tail-body sideslip derivative (calculated previously). Generally, the wing-body contribution to $C_{n_{r}}$ is negligible in comparison to the vertical tail contribution. When the fuselage size is large relative to the wing, the body contribution becomes more important; however, there are no generalized methods to predict the fuselage contribution to yaw damping. Often, for these configurations, the wing alone was sufficient for determining $C_{n_{r}}$ at subsonic speeds. For this study, the calculation of $C_{n_{r}}$ due to the yaw damping of the wing is shown, along with the contribution of the vertical tail. 
The $C_{n_{r}}$ contribution for the wing-body configuration is often small when compared with the vertical tail contribution. Fuselage effects were considered negligible, so the DATCOM method to calculate the contribution from the wings alone was used. Equation 7.42 was used to determine the wing contribution to the yawing moment-due-to-yaw-rate derivative.

$$
\left(C_{n_{r}}\right)_{W}=\left(\frac{C_{n_{r}}}{C_{L}^{2}}\right) C_{L}^{2}+\left(\frac{C_{n_{r}}}{C_{D_{o}}}\right) C_{D_{o}}
$$

where $\frac{C_{n_{r}}}{C_{L}^{2}}$ is the low-speed drag-due-to-lift yaw-damping parameter, $C_{L}$ is the wing lift coefficient, $\frac{C_{n_{r}}}{C_{D_{o}}}$ is the low-speed profile-drag yaw-damping parameter, and $C_{D_{o}}$ is the wing profile drag coefficient evaluated at the appropriate Mach number. $C_{D_{o}}$ is given by Equation 7.43.

$$
C_{D_{o}}=C_{D}+\frac{C_{L}^{2}}{\pi A R}
$$

where $C_{D}$ is the total drag coefficient at the given lift coefficient. For the NACA $1408, C_{L}$ was $0.4, C_{D}$ was 0.074 , and $C_{D_{o}}$ was 0.054 . Using these values, the wing contribution to $C_{n_{r}}$ was found to be -0.029. Once $C_{n_{r}}$ has been calculated for the wing-body, it was necessary to consider the effects of the vertical tail on the yaw damping for a more accurate approximation.

To determine the low-speed drag-due-to-lift yaw-damping parameter, the wing aspect ratio, mean aerodynamic chord, taper ratio, sweep angle at quarter chord, and $\frac{\bar{x}}{\bar{c}}$ were required for use with Figure 105. 


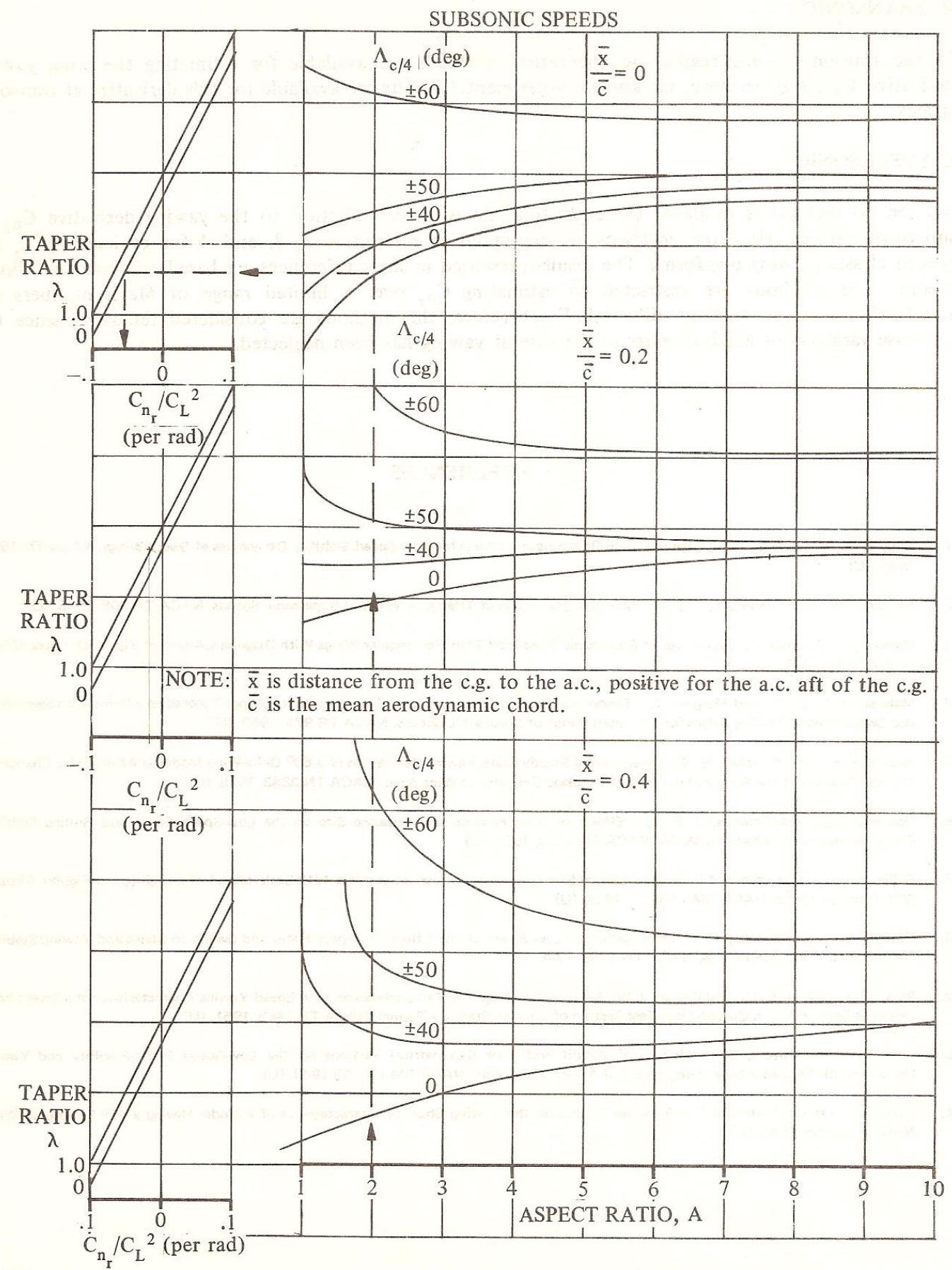

Figure 105: Low-Speed Drag-Due-To-Lift Yaw-Damping Parameter [Reproduced from Reference 41]

The same parameters were required for use with Figure 106 to determine the low-speed profiledrag yaw-damping parameter. 

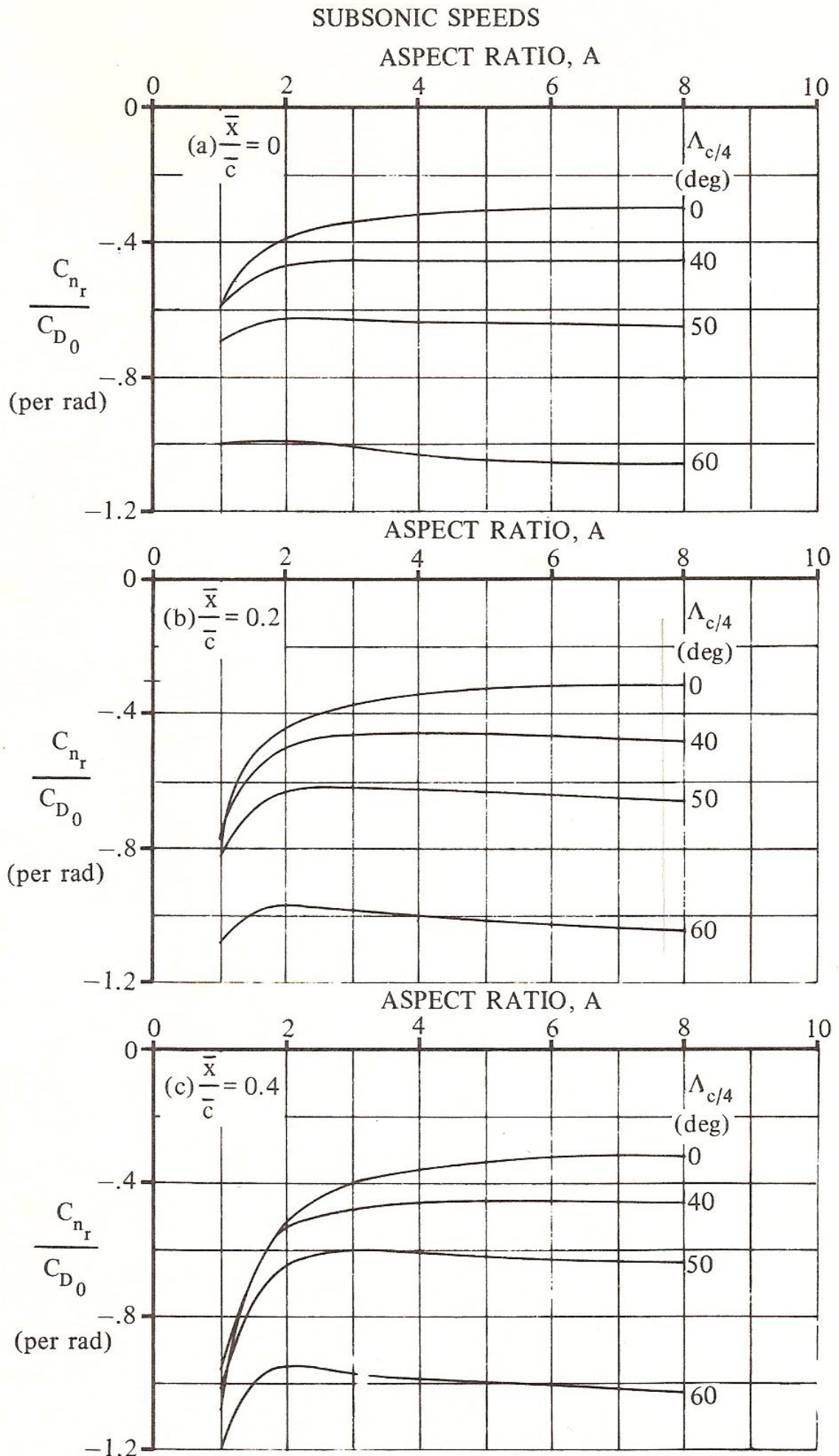

NOTE: $\overline{\mathrm{x}}$ is the distance from the c.g. to the a.c., positive for the a.c. aft of the c.g. $\overline{\mathrm{c}}$ is the wing mean aerodynamic chord.

Figure 106: Low-Speed Profile-Drag Yaw-Damping Parameter [Reproduced from Reference 41] 
These values were implemented back into Equation 7.42 to determine the wing contribution to $C_{n_{r}}$. Next, the vertical tail contribution to the yawing derivative was included for the most accurate results. Finally, using these determined values, the wing-body-tail contribution to $C_{n_{r}}$ was calculated by summing the contributions.

\subsubsection{WVU YF-22 Control Derivatives (DATCOM)}

\subsubsection{Lift-Due-to-Stabilizer-Incidence Derivative: $C_{L_{t H}}$}

The longitudinal control derivative $C_{L_{i H}}$ may be calculated using Equation 7.44.

$$
C_{L_{H H}}=C_{L_{\alpha_{H}}} \eta_{H} \frac{S_{H}}{S}
$$

$C_{L_{\alpha_{H}}}$ was calculated using the Polhamus formula. Equations 7.45and 7.46 were used for the WVU YF-22 calculation.

$$
\begin{aligned}
& k=1+\frac{A R\left(1.87-0.000233 \Lambda_{L E}\right)}{100} \\
& C_{L_{\alpha_{H}}}=\frac{2 \pi A R}{2+\sqrt{\left\{\left[\frac{A R^{2}\left(1-\text { Mach }^{2}\right)}{k^{2}}\left(1+\frac{\tan ^{2}\left(\Lambda_{0.5}\right)}{\left(1-M^{2}\right)}\right)\right]+4\right\}}}
\end{aligned}
$$

\subsubsection{Pitching-Moment-Due-to-Stabilizer-Incidence Derivative: ${ }^{C_{m_{H}}}$}

The longitudinal control derivative $C_{m_{i H}}$ was calculated for the WVU YF-22 using Equation 7.47 .

$$
C_{m_{i H}}=-C_{L_{i H}}\left(\bar{x}_{A C_{H}}-\bar{x}_{C G}\right)=-0.309(1.34)=-0.415
$$

where $\left(\bar{x}_{A C_{H}}-\bar{x}_{C G}\right)$ is the non-dimensional moment arm of the horizontal stabilizer.

\subsubsection{Rolling-Moment-Due-to-Aileron Derivative: $C_{l_{\delta_{A}}}$}

To determine the rolling moment due to aileron deflection $C_{l_{\delta_{A}}}$, a series of steps were followed. First, the change in the inboard and outboard span locations of the ailerons, $\Delta \eta$, was determined as a fraction of half of the wing span. Figure 107 shows the layout that is used to 
determine $\Delta \eta . \beta$ and $\kappa$ are the same as previously represented for the control derivatives, and the value of $\Lambda_{\beta}$ was calculated using Equation 7.49.

$$
\begin{gathered}
\frac{\Delta \eta}{\frac{b}{2}}=\frac{10}{36.245}=0.276 \\
\Lambda_{\beta}=\tan ^{-1}\left(\frac{\tan \Lambda_{c / 4}}{\beta}\right)=\tan ^{-1}\left(\frac{\tan (0.384)}{0.992}\right)=0.387 \quad \text { (per radian) }
\end{gathered}
$$
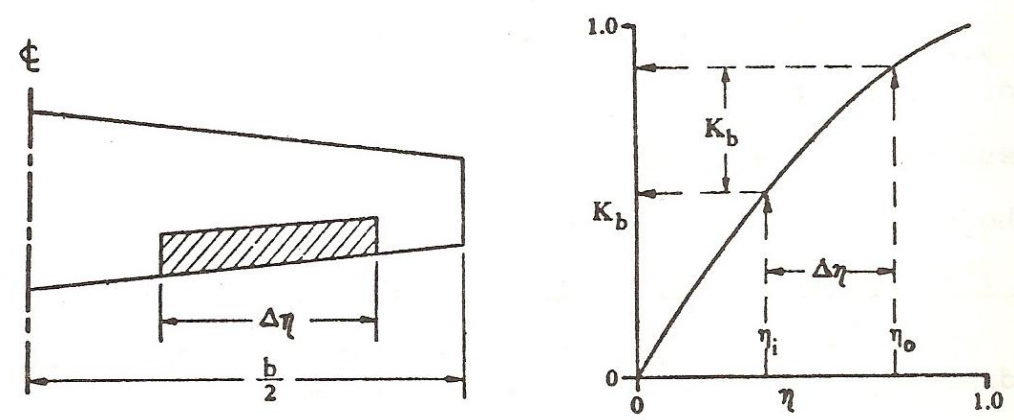

Figure 107: Basic Layout used for Estimating $K_{b}$ [Reproduced from Reference 41]

Next, using Figure 108, $\frac{\beta C l_{l_{\delta}}}{\kappa}$ was calculated to be 0.04 . 


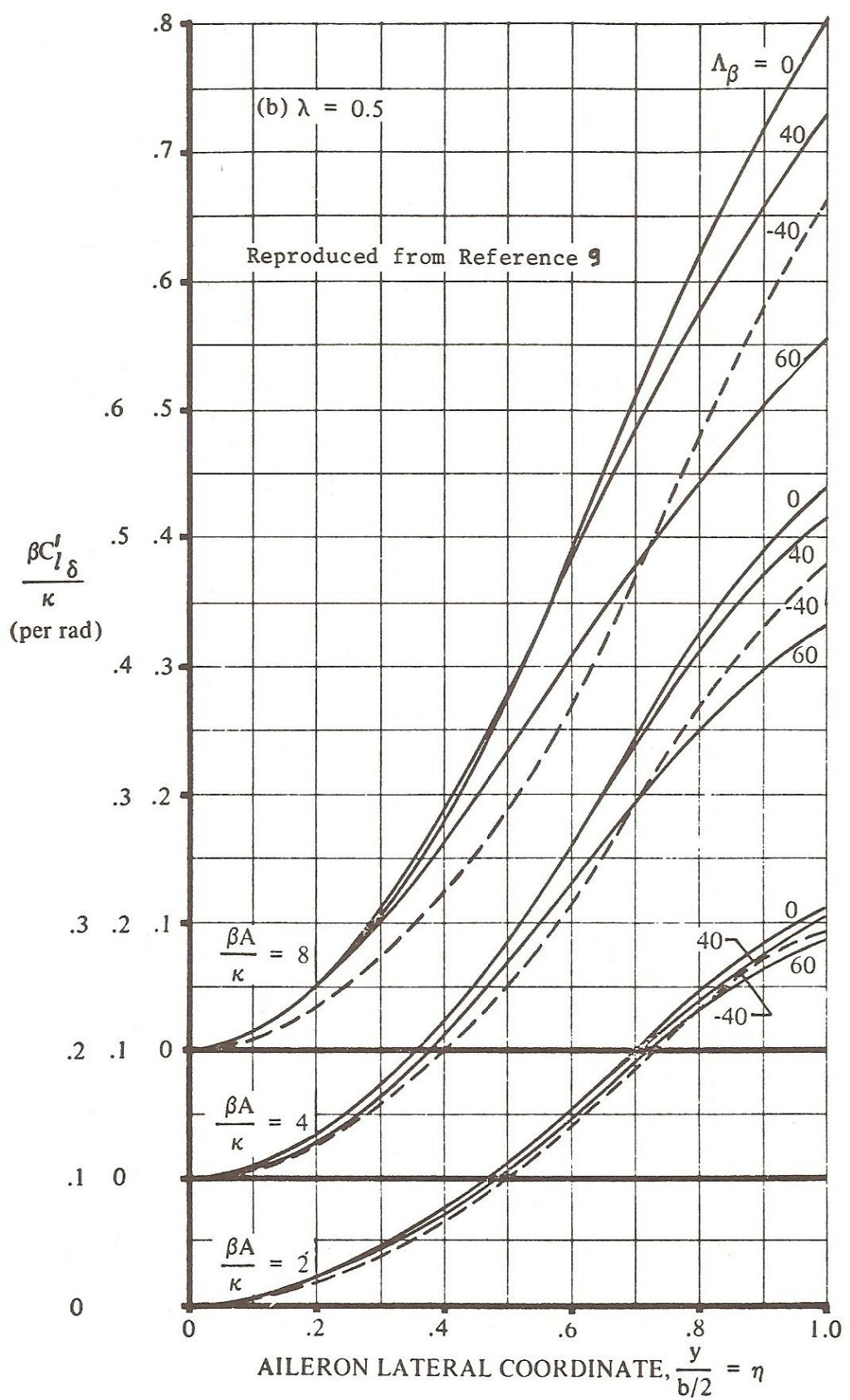

Figure 108: Aileron Rolling Moment Parameter for $\lambda=0.5$ [Reproduced from Reference 41]

Once this was determined, the rolling effectiveness of two full-chord controls anti-symmetrically deflected was calculated by Equation 7.50. 


$$
C^{\prime}{ }_{l_{\delta}}=\left(\frac{\kappa}{\beta}\right) \frac{\beta C_{l_{\delta}}^{\prime}}{\kappa}
$$

Since the WVU YF-22 does not have full-chord controls, the rolling effectiveness of the partialchord controls was determined. The partial-chord control rolling effectiveness, $C_{l_{\delta}}$, was calculated by first determining $c_{l_{\delta}}$ using Equation 7.51.

$$
c_{l_{\delta}}=\left(\frac{c_{l_{\delta}}}{\left(c_{l_{\delta}}\right)_{\text {theory }}}\right)\left(c_{l_{\delta}}\right)_{\text {theory }}
$$

where $\left(\frac{c_{l_{\delta}}}{\left(c_{l_{\delta}}\right)_{\text {theory }}}\right)$ was found using Figure 109 and $\left(c_{l_{\delta}}\right)_{\text {theory }}$ was found from Figure 110.

Thus, $\left(c_{l_{\delta}}\right)_{\text {theory }}$ was found to be 3.1, and $\left(\frac{c_{l_{\delta}}}{\left(c_{l_{\delta}}\right)_{\text {theory }}}\right)$ was determined to be 0.95 .

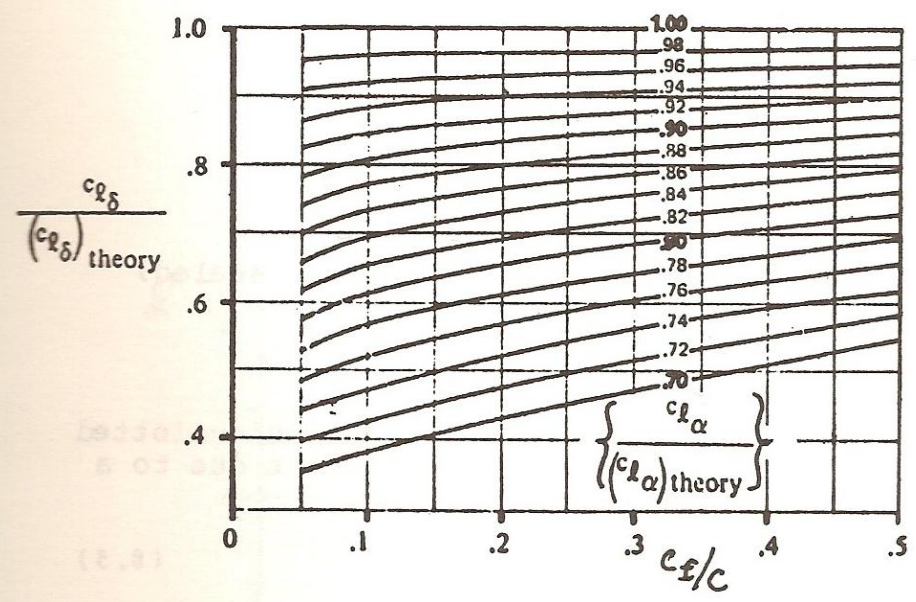

\section{COPIED}

FROM:

REF.9

FOR $C_{l_{\alpha}}$ SEE:

8.1.1.2

FOR $\left(C_{l_{\alpha}}\right)_{\text {theory }}$

SEE: REF 9

SECTION 4.1 .1 .2

OR USE $2 \pi$

Figure 109: Correction Factor for Plain Flap or Aileron Lift [Reproduced from Reference 41] 


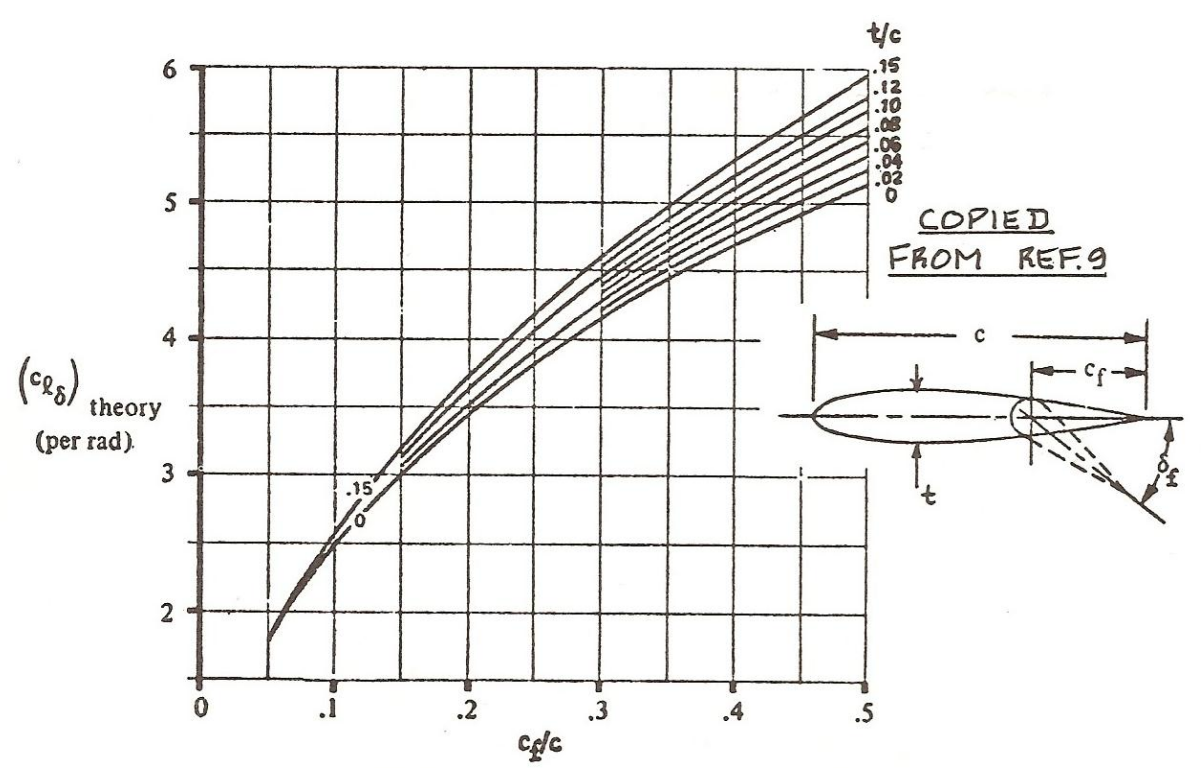

Figure 110: Lift Effectiveness of a Plain Flap or Aileron [Reproduced from Reference 41]

$$
c_{l_{\delta}}=\left(\frac{c_{l_{\delta}}}{\left(c_{l_{\delta}}\right)_{\text {theory }}}\right)\left(c_{l_{\delta}}\right)_{\text {theory }}=0.95(3.1)=2.945
$$

Next, the value of $\alpha_{\delta_{a}}$ was calculated to be 0.4 and used to determine $C_{l_{\delta}}$.

$$
C_{l_{\delta}}=(0.4) 0.039=0.0157
$$

Finally, $C_{l_{\delta_{A}}}$ was calculated using Equation 7.53.

$$
C_{l_{\delta_{A}}}=\left(C_{l_{\delta}}\right)_{\text {left }}+\left(C_{l_{\delta}}\right)_{\text {right }}
$$

\subsubsection{Yawing-Moment-Due-to-Rudder Derivative: $C_{n_{\delta R}}$}

The DATCOM-based method to determine the lateral-directional control derivative $C_{n_{\delta R}}$ was described by the following equation:

$$
C_{n_{\delta R}}=-C_{Y_{\delta R}} \frac{\left(l_{v} \cos \alpha+z_{v} \sin \alpha\right)}{b}
$$

where $C_{Y_{\delta R}}$ is the side-force-due-to-rudder derivative. The vertical tail airfoil on the WVU YF-22 is the NACA 0006. $C_{L_{\alpha_{v}}}$ was calculated using the lift-curve slope of the vertical tail as follows for an $\mathrm{AR}<4$ using Equation 7.55 and the Polhamus formula (Equation 7.56). 


$$
\begin{aligned}
& k=1+\frac{A R\left(1.87-0.000233 \Lambda_{L E}\right)}{100} \\
& C_{L_{\alpha_{v}}}=\frac{2 \pi A R}{2+\sqrt{\left\{\left[\frac{A R^{2}\left(1-M^{2} a c h^{2}\right)}{k^{2}}\left[1+\frac{\tan ^{2}\left(\Lambda_{0.5}\right)}{\left(1-M a c h^{2}\right)}\right)\right]+4\right\}}}
\end{aligned}
$$

Using these values, the derivatives describing the side force and yaw rate due to rudder deflection were determined using Equations 7.57 and 7.58.

$$
\begin{gathered}
C_{Y_{\delta R}}=\left(C_{L_{\alpha V}}\right)\left(k^{\prime} K_{b}\right)\left\lfloor\frac{\left(\alpha_{\delta}\right)_{C_{L}}}{\left(\alpha_{\delta}\right)_{c_{l}}}\right\rfloor\left(\alpha_{\delta}\right)_{c_{l}} \frac{S_{V}}{S} \\
C_{n_{\delta R}}=-C_{Y_{\delta R}} \frac{\left(l_{v} \cos \alpha+z_{v} \sin \alpha\right)}{b}
\end{gathered}
$$

\subsection{PACT DATCOM Analysis}

A modeling study for the PACT was conducted using USAF DATCOM and Empirical methods (Polhamus Formula). The values obtained from DATCOM were implemented by helping to determine bounds in an optimization scheme for the stability and control derivatives determined from PID. A comparison of the results obtained from the PID study and the DATCOM/Empirical analysis was also completed. The following sections describe the process and equations that were utilized for the analysis of the WVU PACT through DATCOM and Empirical methodologies.

\subsubsection{PACT Downwash Calculation}

The following equations were used to determine the downwash gradient, $\frac{d \varepsilon}{d \alpha}$, which represents the downwash experienced by the horizontal tail.

$$
\frac{d \varepsilon}{d \alpha}=4.44\left(\left(K_{A R} K_{\lambda} K_{H}\right)\left(\cos \Lambda_{c / 4}\right)^{1 / 2}\right)^{1.19} \frac{\left(c_{L_{\alpha_{w}}}\right)_{a t M}}{\left(c_{L_{\alpha_{w}}}\right)_{a t M=0}}
$$

where Equations $7.60-7.62$ represent the factors for calculating the downwash. 


$$
\begin{gathered}
K_{A R}=\left(\frac{1}{A R}\right)-\frac{1}{\left(1+A R^{1.7}\right)} \\
K_{\lambda}=\frac{(10-3 \lambda)}{7} \\
K_{H}=\frac{\left(\frac{1-h_{H}}{b}\right)}{\left(\frac{2 l_{H}}{b}\right)^{1 / 3}}
\end{gathered}
$$

Figure 111 shows the DATCOM tables that were used to determine the constants for the PACT downwash gradient calculation.

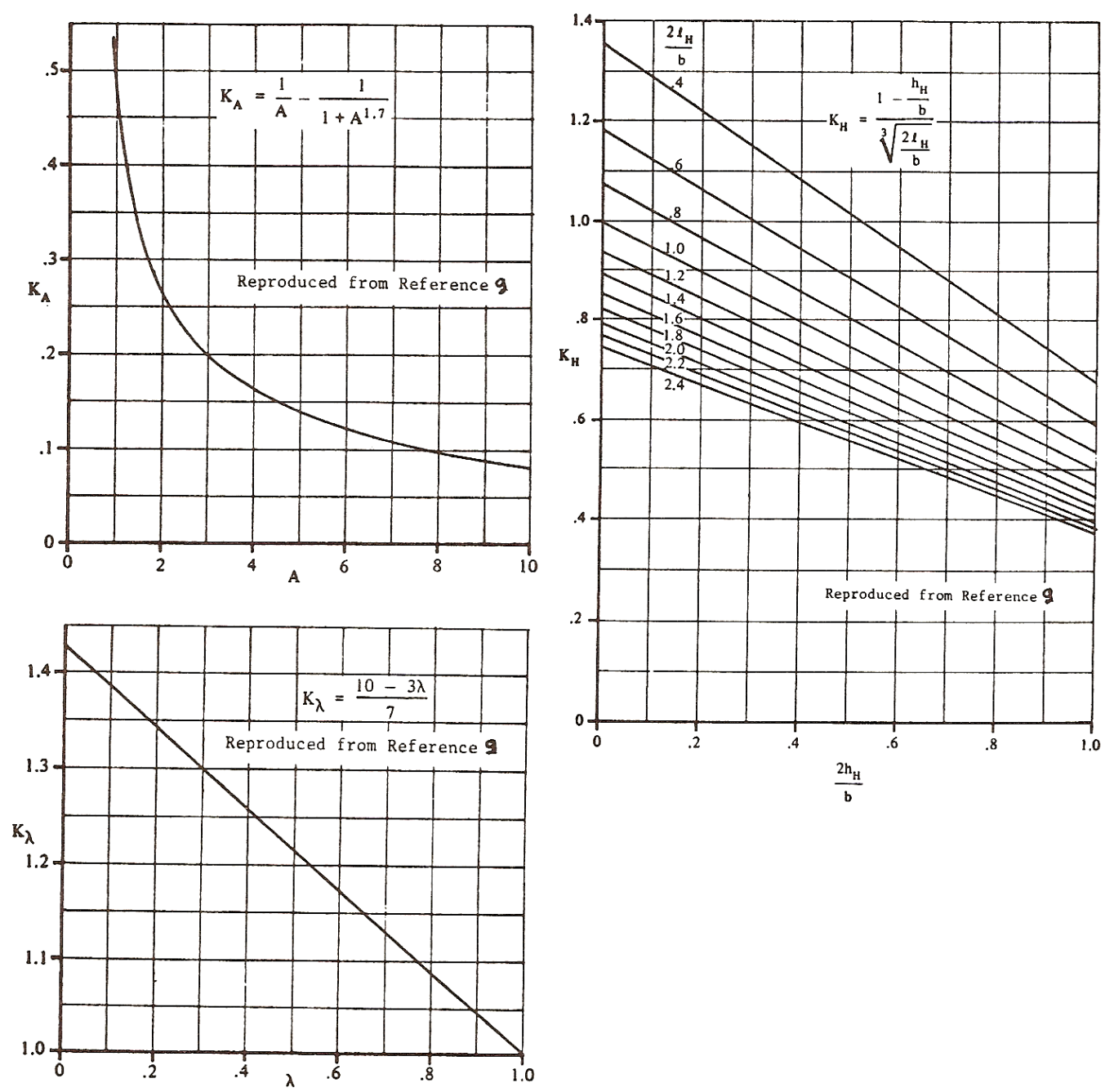

Figure 111: Factors for Computing Downwash [Reproduced from Reference 42] 


\subsubsection{PACT Stability Derivatives (DATCOM)}

\subsubsection{Zero-Lift Drag Derivative: $c_{D_{0}}$}

The total zero-lift drag derivative was calculated by summing the contributions from the wing, fuselage, and empennage surfaces (i.e. horizontal and vertical tails) as shown in Equation 7.63.

$$
c_{D_{0}}=c_{D_{D_{W}}}+c_{D_{0_{f}}}+c_{D_{0_{E}}}
$$

To calculate the zero-lift drag for the wing, Equation 7.64 was used, which required knowledge of the wetted area of the wings as well as the airfoil thickness parameter $L^{\prime}$ and the thickness ratio at the mean geometric chord of the wing $(t / c)$. For the PACT, $(t / c)$ is 0.099 .

$$
c_{D_{0_{w}}}=\left(R_{w f}\right)\left(R_{L S}\right)\left(c_{f_{w}}\right)\left(1+L^{\prime}\left(\frac{t}{c}\right)+100\left(\frac{t}{c}\right)^{4}\right)\left(\frac{S_{w^{2} t_{w}}}{S}\right)
$$

where $\left(R_{w f}\right)$ is the wing/fuselage interference factor found from Figure 112, $\left(R_{L S}\right)$ is the lifting surface correction factor found from Figure 113, and $\left(c_{f_{w}}\right)$ is the turbulent flat plate skin-friction coefficient of the wing found from Figure 114.

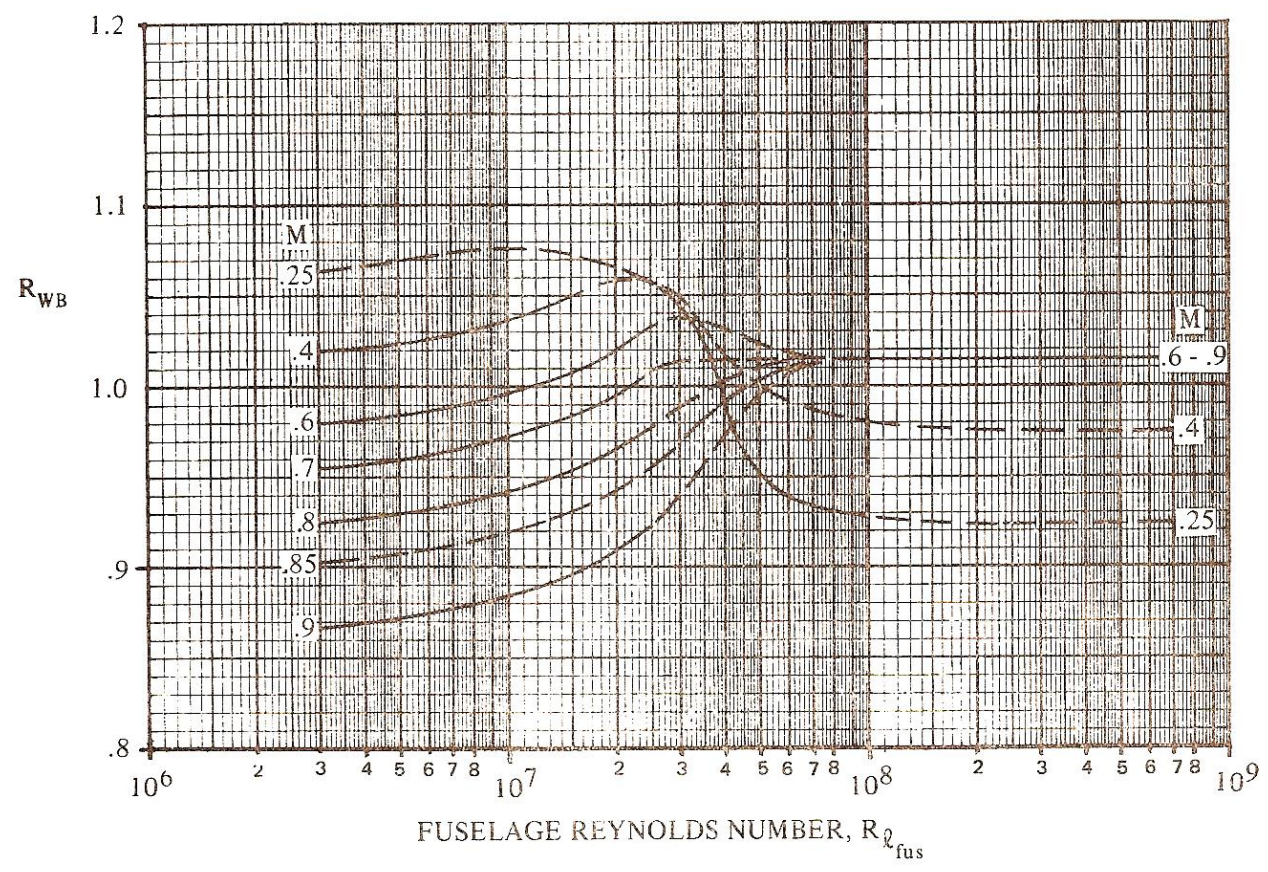

Figure 112: Wing-Fuselage Interference Correlation Factor [Reproduced from Reference 41] 


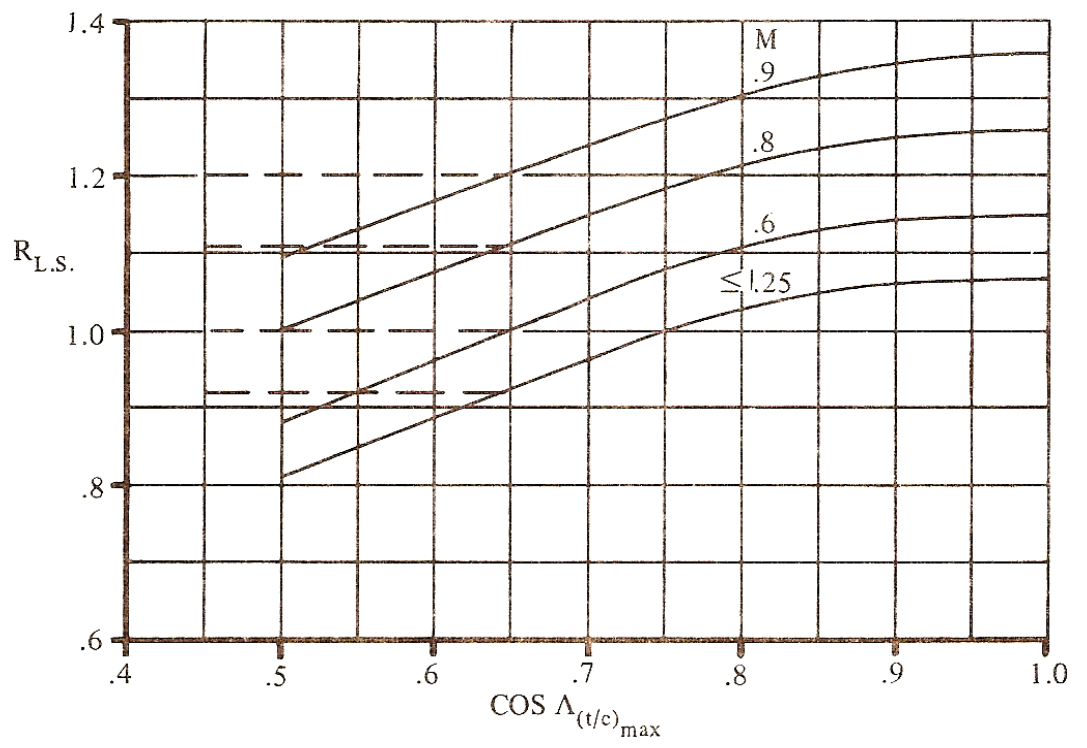

Figure 113: Lifting Surface Correlation Factor for Subsonic Minimum Drag [Reproduced from Reference 41]

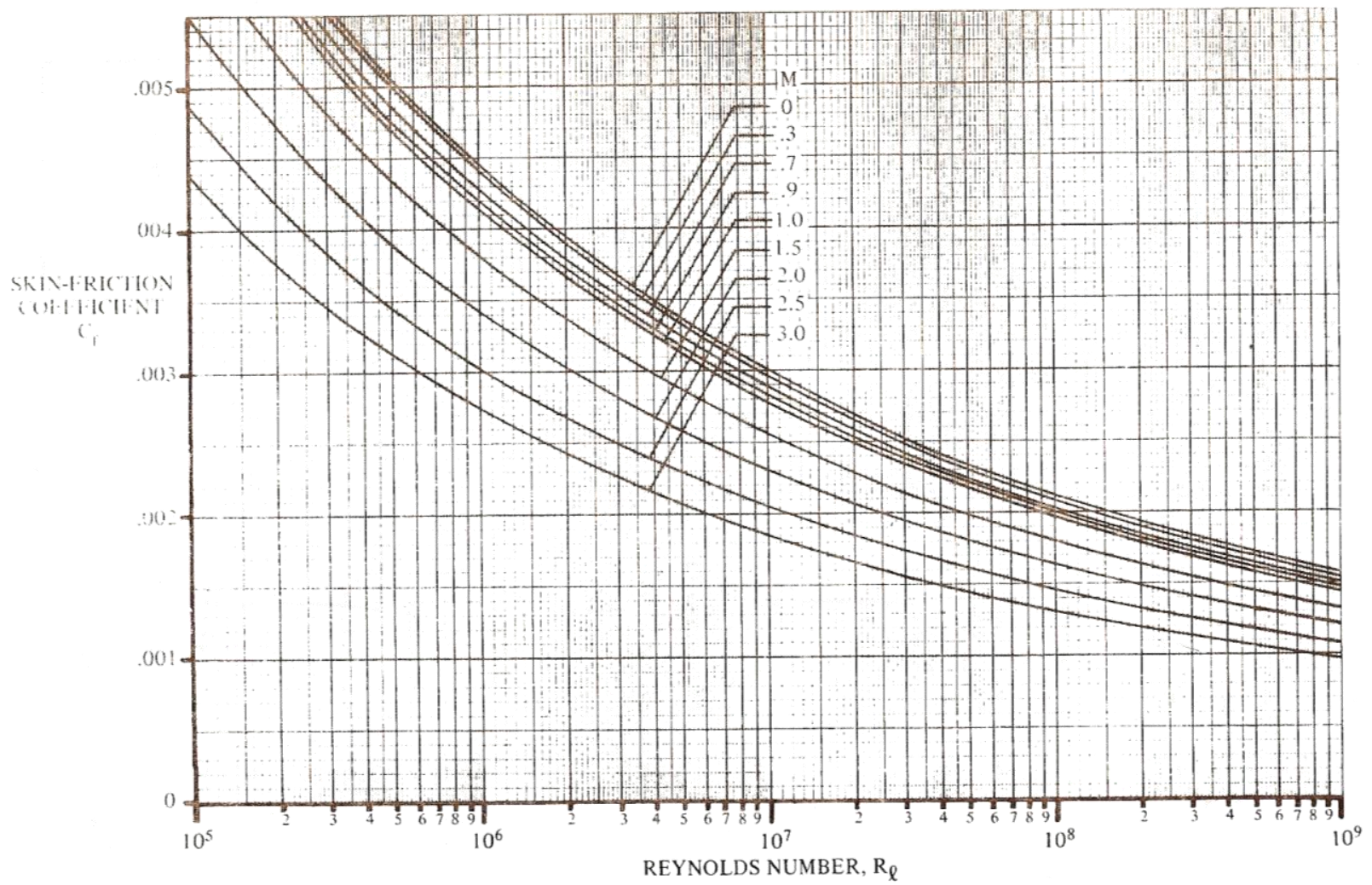

Figure 114: Turbulent Mean Skin-Friction Coefficient of Insulated Flat Plate [Reproduced from Reference 41]

The airfoil thickness location parameter was obtained using the following criteria:

$$
\begin{aligned}
L^{\prime} & =1.2 \text { for }(\mathrm{t} / \mathrm{c})_{\max } \text { at } x_{t} \geq 0.3 c \\
L^{\prime} & =2.0 \text { for }(\mathrm{t} / \mathrm{c})_{\max } \text { at } x_{t}<0.3 c
\end{aligned}
$$


The fuselage zero-lift drag coefficient was found using Equation 7.65.

$$
c_{D_{0_{f}}}=R_{w f} c_{f_{\text {fis }}}\left(1+\frac{60}{\left(\frac{l_{f}}{d_{f}}\right)^{3}}+0.0025\left(\frac{l_{f}}{d_{f}}\right)\right)\left(\frac{S_{w_{\text {fet fus }}}}{S}\right)+C_{D_{b_{\text {futs }}}}
$$

where $c_{f_{f u s}}$ is the turbulent flat plate skin-friction coefficient of the fuselage, $l_{f}$ is the fuselage length, and $d_{f}$ is the maximum fuselage diameter. The Reynolds number for the flow of air over the fuselage was calculated by Equation 7.66.

$$
\operatorname{Re}_{\text {fus }}=\frac{\rho U l_{f}}{\mu}
$$

The empennage zero-lift drag coefficient was calculated for the surfaces of the empennage using Equation 7.64. The appropriate parameters were replaced with data reflecting those of either the horizontal or vertical tail surfaces. In the case of the PACT, $\left(R_{w f}\right)$ is equal to a value of 1.0 .

\subsubsection{Drag-Due-to-Angle-of-Attack Derivative: ${ }^{c_{D_{\alpha}}}$}

The drag-due-to-angle-of-attack derivative was calculated using Equation 7.67, which can be used for aircraft with parabolic drag polars ${ }^{42}$.

$$
c_{D_{\alpha}}=\left(\frac{2 c_{L_{1}}}{\pi A \operatorname{Re}}\right) c_{L_{\alpha}}
$$

where $e$ is the Oswald efficiency and $c_{L_{1}}$ was approximated using Equation 7.68 for steady level flight.

$$
c_{L_{1}}=\frac{W}{\frac{1}{2} \rho V^{2} S}
$$

\subsubsection{Drag-Due-to-Pitch-Rate Derivative: $c_{D_{q}}$}

The drag-due-to-pitch-rate derivative is negligible ${ }^{42}$, so it wa set equal to zero for this aircraft.

$$
c_{D_{q}}=0
$$

\subsubsection{Zero-Lift Lift Derivative: ${ }^{c_{L_{0}}}$}

The zero-lift lift derivative was obtained from the airfoil profile of the aircraft wing. For the PACT aircraft, the NACA 2410 airfoil was used. 


\subsubsection{Lift-Due-to-Angle-of-Attack Derivative: ${ }^{c_{L_{\alpha}}}$}

The lift-due-to-angle-of-attack derivative (lift-curve slope) was estimated for the PACT aircraft using Equation 7.70.

$$
c_{L_{\alpha}}=c_{L_{\alpha_{w f}}}+c_{L_{\alpha_{H}}} \eta_{H}\left(\frac{S_{H}}{S}\right)\left(1-\frac{d \varepsilon}{d \alpha}\right)
$$

The wing-fuselage lift curve slope was calculated using Equation 7.71.

$$
c_{L_{\alpha_{w f}}}=K_{w f} c_{L_{\alpha_{w}}}
$$

where $K_{w f}$ is the wing-fuselage interference factor as calculated by Equation 7.72.

$$
K_{w f}=1+0.025\left(\frac{d_{f}}{b}\right)-0.25\left(\frac{d_{f}}{b}\right)^{2}
$$

\subsubsection{Lift-Due-to-Pitch-Rate Derivative: $c_{L_{q}}$}

The lift-due-to-pitch-rate derivative was calculated with Equation 7.73, which used the wing and horizontal tail contributions.

$$
c_{L_{q}}=c_{L_{q_{w}}}+c_{L_{q_{H}}}
$$

The wing contribution (average wing airfoil lift-curve slope) to the total derivative was calculated using Equation 7.74, which required the wing aspect ratio and quarter-chord sweep angle.

$$
c_{L_{q_{w}}}=\frac{A R+2 \cos \Lambda_{c / 4}}{(A R) B+2 \cos \Lambda_{c / 4}}\left(c_{L_{q_{W}}}\right)_{M=0}
$$

where $\left(c_{L_{q_{w}}}\right)_{M=0}$ is defined by Equation 7.75 .

$$
\left(c_{L_{q_{w}}}\right)_{M=0}=\left(0.5+\frac{2 x_{w}}{\bar{c}}\right) c_{l_{\alpha_{w}}}
$$

where $x_{w}$ is defined for the PACT by Figure 115, and where $c_{l_{\alpha_{w}}}$ is the average wing airfoil liftcurve-slope. The parameter $B$ used in Equation 7.74 is a function of the Mach number and geometry of the aircraft wing and is calculated using Equation 7.76.

$$
B=\sqrt{1-M^{2} \cos ^{2} \Lambda_{c / 4}}
$$




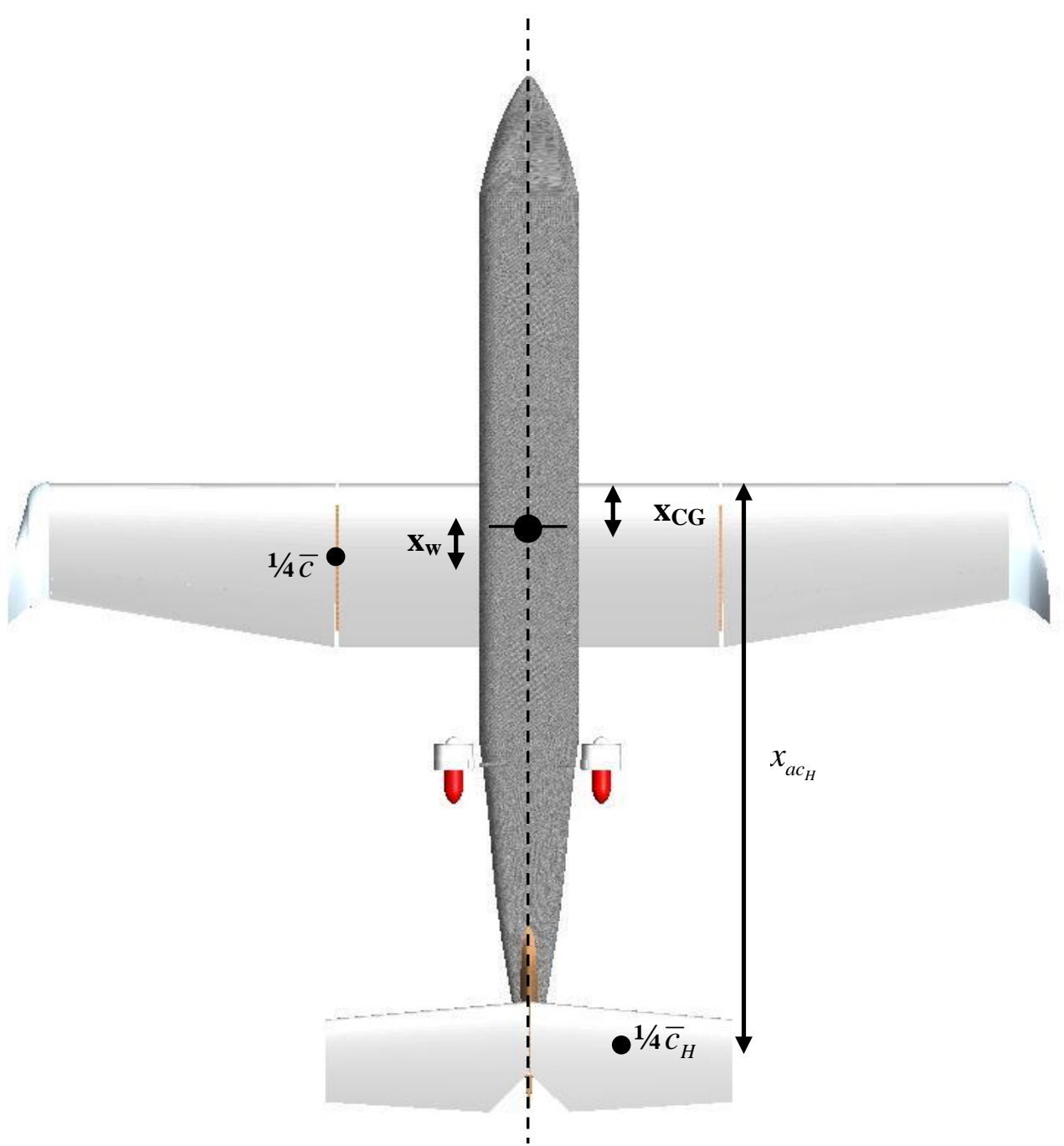

Figure 115: Definition of Required PACT Geometric Parameters

The horizontal tail contribution to the lift-due-to-pitch-rate derivative was calculated using Equation 7.77, which requires geometric parameters for the PACT defined in Figure 115.

$$
c_{L_{q H}}=2 c_{L_{\alpha_{H}}} \eta_{H} \frac{S_{H}}{S}\left(\bar{x}_{a c_{H}}-\bar{x}_{C G}\right)
$$

\subsubsection{Zero-Lift Pitching Moment Derivative: $c_{m_{0}}$}

The zero-lift pitching moment derivative was obtained from the NACA 2410 airfoil characteristics listed in References 41 and 91.

\subsubsection{Pitching-Moment-Due-to-Angle-of-Attack Derivative: $c_{m_{\alpha}}$}

The pitching-moment-due-to-angle-of-attack (static longitudinal stability) derivative for the PACT aircraft was calculated using Equation 7.78 for all flight speed regimes. 


$$
c_{m_{\alpha}}=\left(\frac{d c_{m}}{d c_{L}}\right) c_{L_{\alpha}}
$$

where

$$
\left(\frac{d c_{m}}{d c_{L}}\right)=\bar{x}_{r e f}-\bar{x}_{a c_{A}}
$$

where $\bar{x}_{\text {ref }}$ is the location of the moment reference center in fractions of the mean geometric chord. The power effects from the engines may also need to be considered in the calculation of the pitching-moment-due-to-angle-of-attack derivative. These power effects that may need to be accounted for include the power effect caused by thrustline offset or propeller slipstream and the propeller/inlet normal forces ${ }^{42}$.

\subsubsection{Pitching-Moment-Due-to-Pitch-Rate Derivative: $c_{m_{q}}$}

The total pitching-moment-due-to-pitch-rate derivative was estimated by summing the contributions of the wing and horizontal tail for the PACT aircraft, as shown by Equation 7.80.

$$
c_{m_{q}}=c_{m_{q_{w}}}+c_{m_{q_{H}}}
$$

The wing contribution was determined from Equation 7.81, which required the wing aspect ratio, quarter-chord sweep angle, and parameter $B-$ a function of Mach number.

$$
c_{m_{q_{w}}}=c_{m_{q_{w a t} M=0}}\left(\frac{\frac{(A R)^{3} \tan ^{2} \Lambda_{c / 4}}{(A R) B+6 \cos \Lambda_{c / 4}}+\frac{3}{B}}{\frac{(A R)^{3} \tan ^{2} \Lambda_{c / 4}}{A R+6 \cos \Lambda_{c / 4}}+3}\right)
$$

The parameter $c_{m_{q_{\text {wat } M=0}}}$ was calculated using Equation 7.82 .

$$
\left(c_{m_{q_{w}}}\right)_{M=0}=-K_{w} c_{l_{\alpha_{w}}} \cos \Lambda_{c / 4}\left(\left(\frac{A R\left(2\left(\frac{x_{w}}{\bar{c}}\right)^{2}+0.5\left(\frac{x_{w}}{\bar{c}}\right)\right)}{A R+2 \cos \Lambda_{c / 4}}\right)+\left(\frac{(A R)^{3} \tan ^{2} \Lambda_{c / 4}}{24\left(A R+6 \cos \Lambda_{c / 4}\right)}\right)+\frac{1}{8}\right)
$$

where $K_{w}$ is the correction constant for wing contribution to pitch damping as found by Figure 116. 


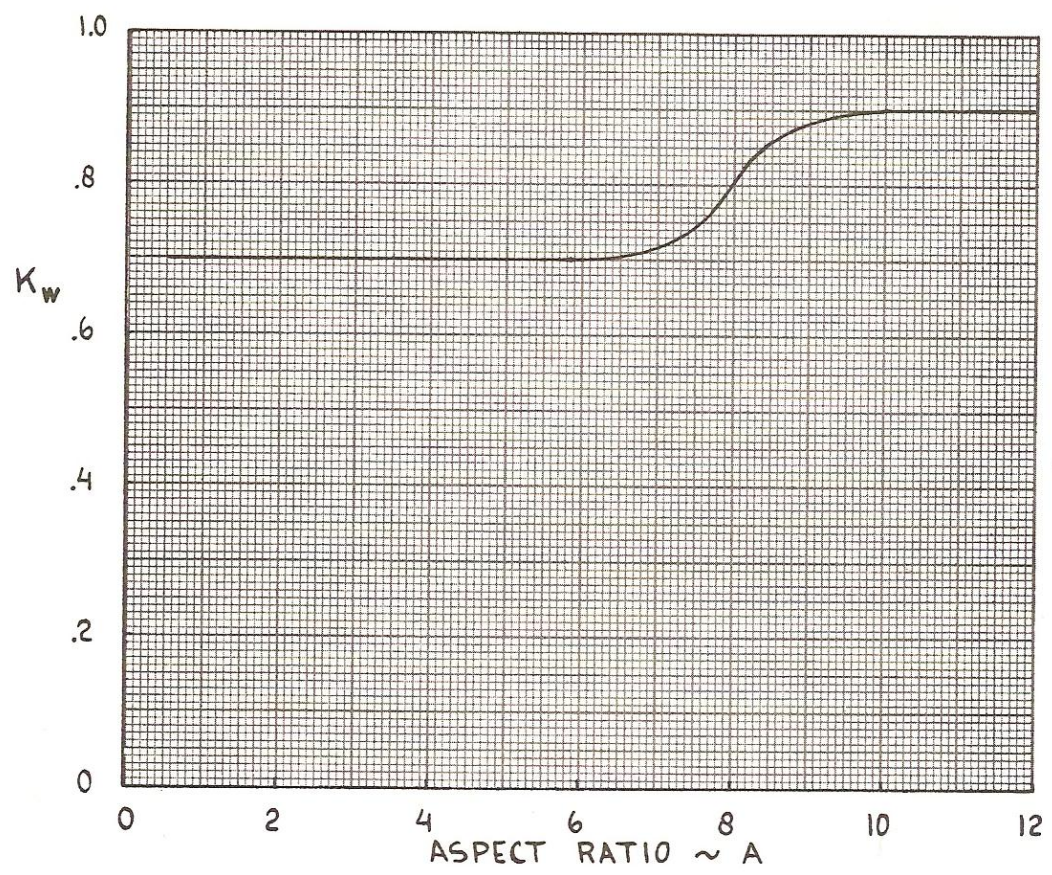

Figure 116: Correction Constant for Wing Contribution to Pitch Damping [Reproduced from Reference 41]

The horizontal tail contribution was calculated using Equation 7.83, with geometric parameters defined by Figure 115.

$$
c_{m_{q_{H}}}=-2 c_{l_{\alpha_{H}}} \eta_{H} \frac{S_{H}}{S}\left(\bar{x}_{a c_{H}}-\bar{x}_{C G}\right)^{2}
$$

\subsubsection{Side-Force-Due-to-Sideslip Derivative: $c_{Y_{\beta}}$}

The side-force-due-to-sideslip derivative was estimated using Equation 7.84, which accounted for the wing, fuselage, and vertical tail contributions.

$$
c_{Y_{\beta}}=c_{Y_{\beta_{w}}}+c_{Y_{\beta_{f}}}+c_{Y_{\beta_{v}}}
$$

The wing contribution was estimated using Equation 7.85, which required the wing geometric dihedral of the aircraft, $\Gamma$, in degrees. For the PACT aircraft, the geometric dihedral is equal to zero.

$$
c_{Y_{\beta_{w}}}=-0.00573(|\Gamma|)
$$

The fuselage contribution was estimated using Equation 7.86, which required the cross-sectional area of the fuselage, $S_{o}$, to be defined where the flow was no longer considered potential. 


$$
c_{Y_{\beta_{f}}}=-2 K_{i}\left(\frac{S_{o}}{S}\right)
$$

where $K_{i}$ is the wing-fuselage interference factor found from Figure 117.

ALL SPEELS

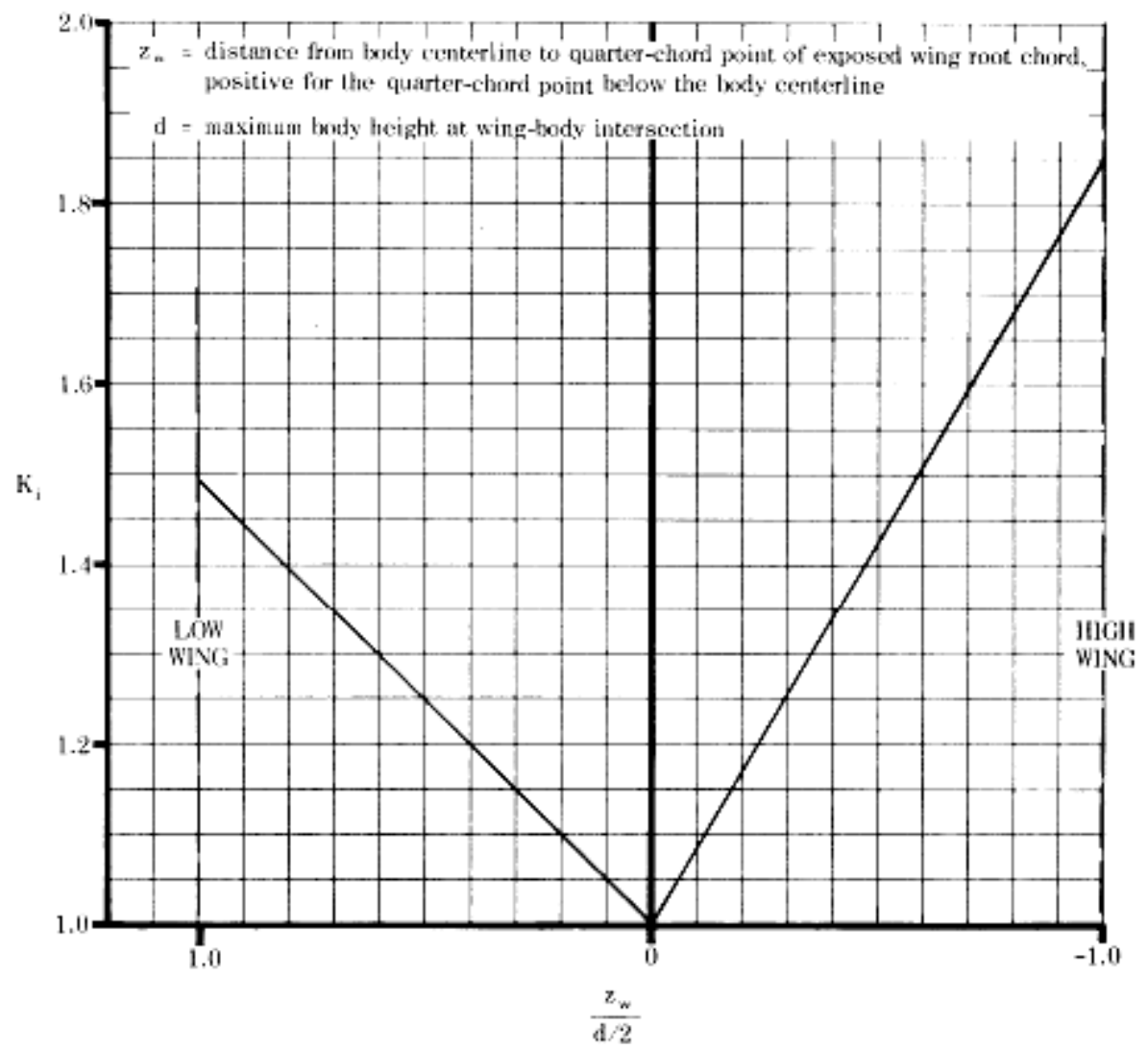

Figure 117: Wing-Body Interference Factor [Reproduced from Reference 42]

The vertical tail contribution was determined based on the type of tail setup for the aircraft. Calculations were completed for a single vertical tail for the PACT aircraft using Equation 7.87

$$
c_{Y_{\beta_{v}}}=-k_{v}\left(c_{L_{\alpha_{v}}}\right)\left(1+\frac{d \sigma}{d \beta}\right) \eta_{v}\left(\frac{S_{v}}{S}\right)
$$

where $k_{v}$ is an empirical factor found from Figure 118. 


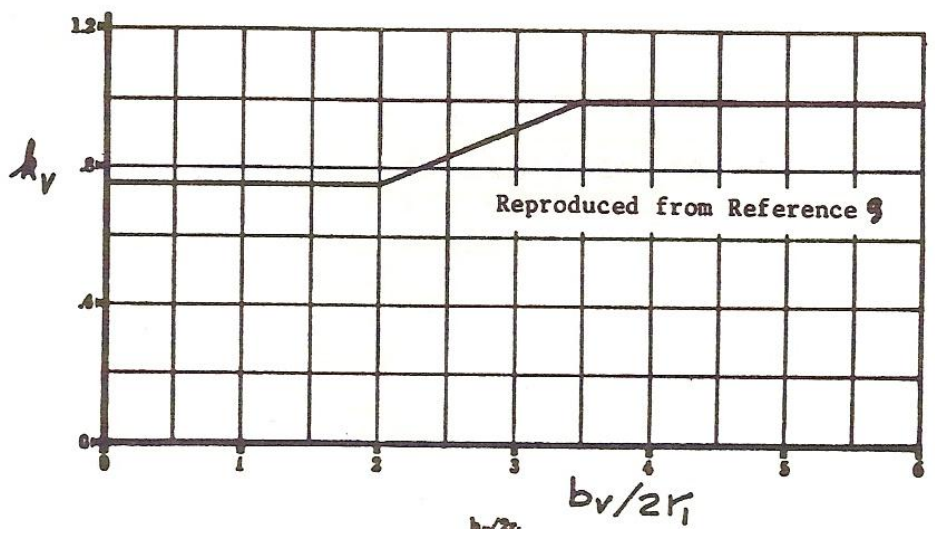

Figure 118: Empirical Factor for Estimating Side-Force due to Sideslip of a Single Vertical Tail [Reproduced from Reference 42]

The derivative $c_{L_{\alpha_{\nu}}}$ was calculated using Equation 7.88, using the appropriate parameters corresponding to the vertical tail.

$$
c_{L_{\alpha_{v}}}=\frac{2 \pi A R}{\left[2+\left\{\frac{A R^{2} \beta^{2}}{k^{2}}\left(\frac{1+\tan ^{2} \Delta_{c / 2}}{\beta^{2}}\right)+4\right\}^{1 / 2}\right]}
$$

where

$$
\beta=\sqrt{1-M^{2}}
$$

The value of $\left(1+\frac{d \sigma}{d \beta}\right) \eta_{v}$ was estimated using Equation 7.90, where $S_{v}$ is the effective vertical tail area and $A R$ is the vertical tail aspect ratio.

$$
\left(1+\frac{d \sigma}{d \beta}\right) \eta_{v}=0.724+3.06\left(\frac{\left(\frac{S_{v}}{S}\right)}{1+\cos \Lambda_{c / 4}}\right)+0.4\left(\frac{z_{w}}{z_{f}}\right)+0.009 A R
$$

where $z_{f}$ and $z_{w}$ are defined by Figure 119 . 

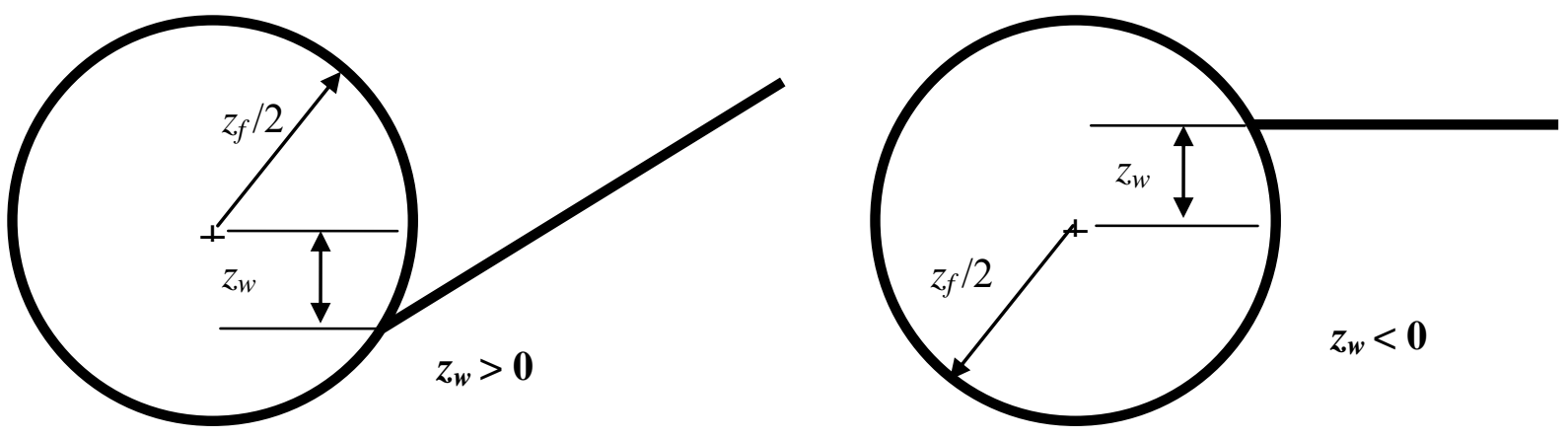

Figure 119: Definition of Wing-Fuselage Parameters for Low and High Wing Aircraft ${ }^{41}$

\subsubsection{Side-Force-Due-to-Roll-Rate Derivative: ${ }^{c_{Y_{p}}}$}

The side-force-due-to-roll-rate derivative is mainly influenced by the vertical tail ${ }^{42}$. This derivative was estimated using Equation 7.91, where $l_{v}, z_{v}$, and $\alpha$ are geometric parameters defined using Figure 120, and $b$ is the vertical tail span.

$$
c_{Y_{p}}=2\left(c_{Y_{\beta_{v}}}\right) \frac{\left(z_{v} \cos \alpha-l_{v} \sin \alpha\right)}{b}
$$

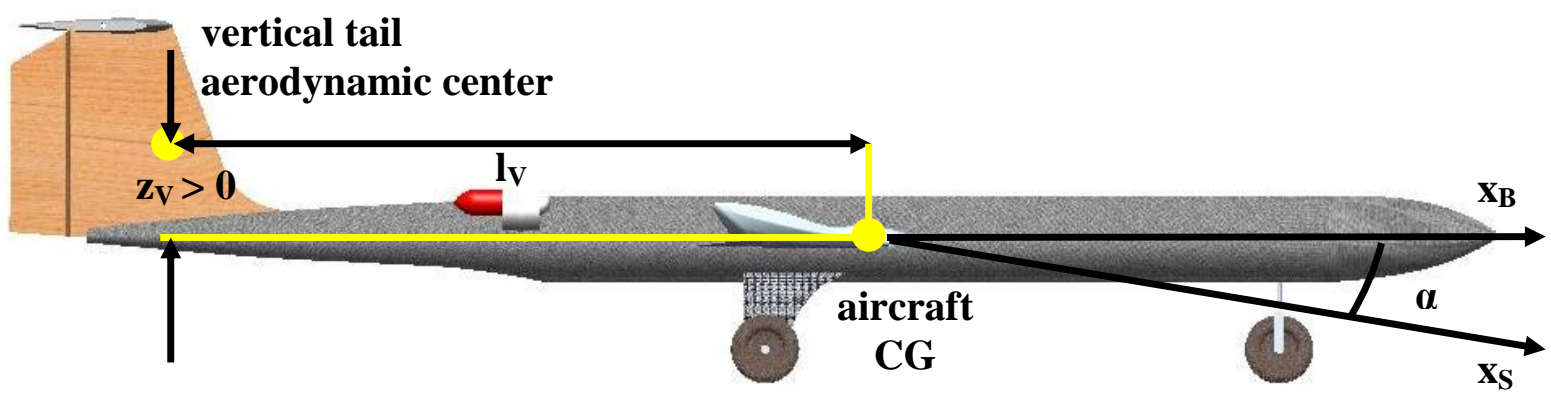

Figure 120: PACT Geometry for Locating Vertical Tail

\subsubsection{Side-Force-Due-to-Yaw-Rate Derivative: ${ }^{c_{Y_{p}}}$}

The side-force-due-to-yaw-rate derivative is mainly influenced by the vertical tail ${ }^{42}$ and was estimated with Equation 7.92 where $l_{v}, z_{v}$, and $\alpha$ are geometric parameters defined using Figure 120 , and $b$ is the vertical tail span. 


$$
c_{Y_{r}}=-2\left(c_{Y_{\beta_{v}}}\right) \frac{\left(l_{v} \cos \alpha+z_{v} \sin \alpha\right)}{b}
$$

\subsubsection{Rolling-Moment-Due-to-Sideslip Derivative: $c_{l_{\beta}}$}

The rolling-moment-due-to-sideslip derivative was estimated using Equation 7.93, which required the contributions from the wing-body, horizontal tail, and vertical tail.

$$
c_{l_{\beta}}=c_{l_{\beta_{w}}}+c_{l_{\beta_{H}}}+c_{l_{\beta_{v}}}
$$

The wing-body contribution was estimated by Equation 7.94.

$$
c_{l_{\beta_{w f}}}=57.3\left(\begin{array}{c}
\left.c_{L_{w f}}\left(\left(\frac{c_{l_{\beta}}}{c_{L}}\right)_{\Lambda_{c / 2}}\left(K_{M_{\Lambda}}\right)\left(K_{f}\right)+\left(\frac{c_{l_{\beta}}}{c_{L}}\right)_{A R}\right)+\Gamma\left(\left(\frac{c_{l_{\beta}}}{\Gamma}\right) K_{M_{\Gamma}}+\left(\frac{\Delta c_{l_{\beta}}}{\Gamma}\right)\right)\right) \\
+\left(\Delta c_{l_{\beta}}\right)_{z_{w}}+\left(\varepsilon_{t} \tan \Lambda_{c / 4}\right)\left(\frac{\Delta c_{l_{\beta}}}{\varepsilon_{t} \tan \Lambda_{c / 4}}\right)
\end{array}\right)
$$

where $\left(\frac{c_{l_{\beta}}}{c_{L}}\right)_{\Lambda_{c / 2}}$ is the wing sweep contribution found from Figure 121, $K_{M_{\Lambda}}$ is the compressibility correction to sweep determined from Figure 122, $K_{f}$ is a fuselage correction factor found from Figure 124 utilizing the geometric parameters of the PACT aircraft defined in Figure 123, $\left(\frac{c_{l_{\beta}}}{c_{L}}\right)_{A R}$ is the aspect ratio contribution obtained from Figure $125, \Gamma$ is the geometric dihedral angle of the wing, $\left(\frac{c_{l_{\beta}}}{\Gamma}\right)$ is the wing dihedral effect found from Figure $126, K_{M_{\Gamma}}$ is the compressibility correction to dihedral found from Figure $127,\left(\frac{\Delta c_{l_{\beta}}}{\Gamma}\right)$ is the fuselage induced effect on the wing height calculated using Equation 7.95, $\left(\Delta c_{l_{\beta}}\right)_{z_{w}}$ is calculated using Equation 7.97, and $\left(\frac{\Delta c_{l_{\beta}}}{\varepsilon_{t} \tan \Lambda_{c / 4}}\right)$ is the wing twist correction factor found from Figure 128.

$$
\left(\frac{\Delta c_{l_{\beta}}}{\Gamma}\right)=-0.0005 A R\left(\frac{d_{f_{\text {avg }}}}{b}\right)^{2}
$$

where 


$$
\begin{gathered}
d_{f_{\text {avg }}}=\sqrt{\frac{S_{\text {avg fuse CS }}}{0.7548}} \\
\left(\Delta c_{l_{\beta}}\right)_{z_{w}}=0.042 \sqrt{A R}\left(\frac{z_{w}}{b}\right)\left(\frac{d_{f_{\text {avg }}}}{b}\right)
\end{gathered}
$$

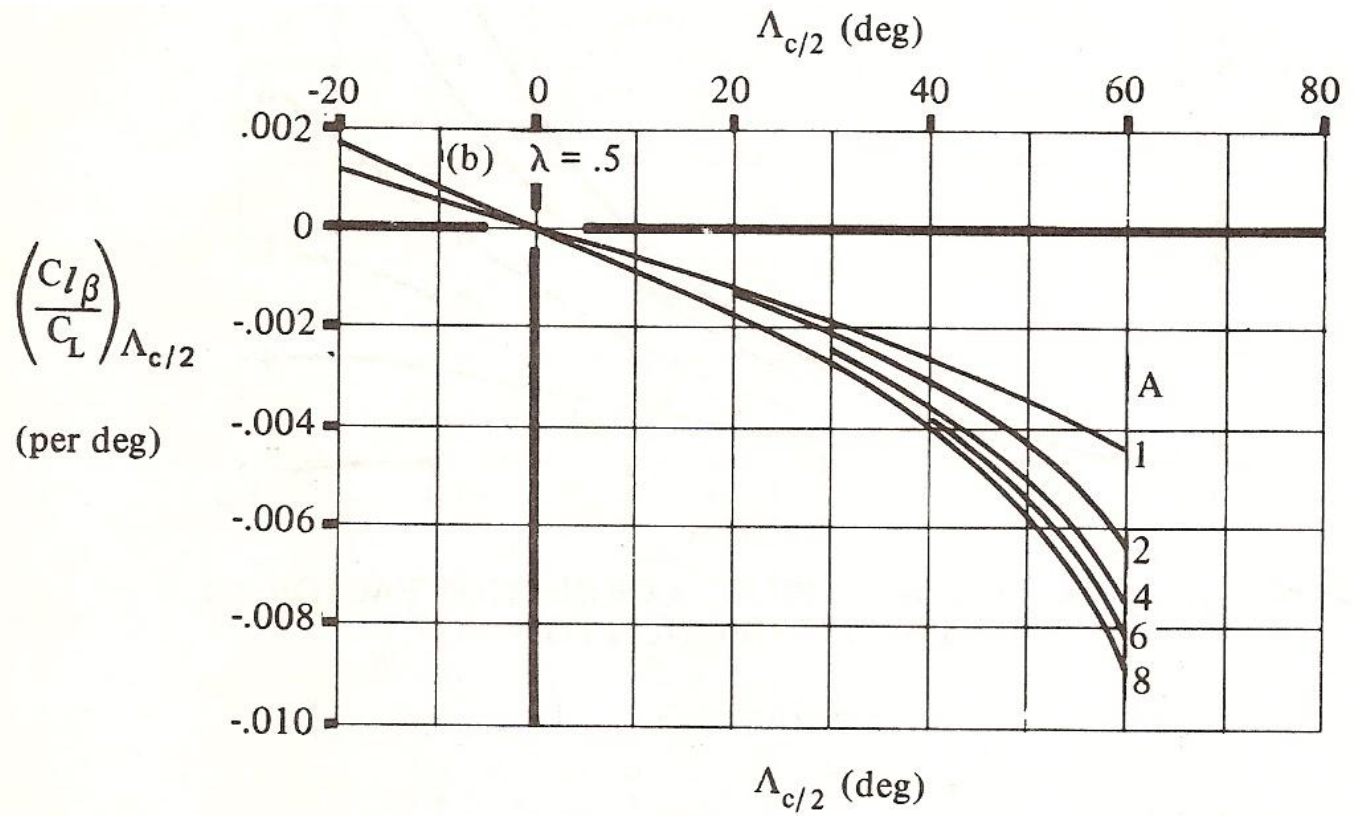

Figure 121: Wing Sweep Contribution to Rolling Moment due to Sideslip [Reproduced from Reference 42] 


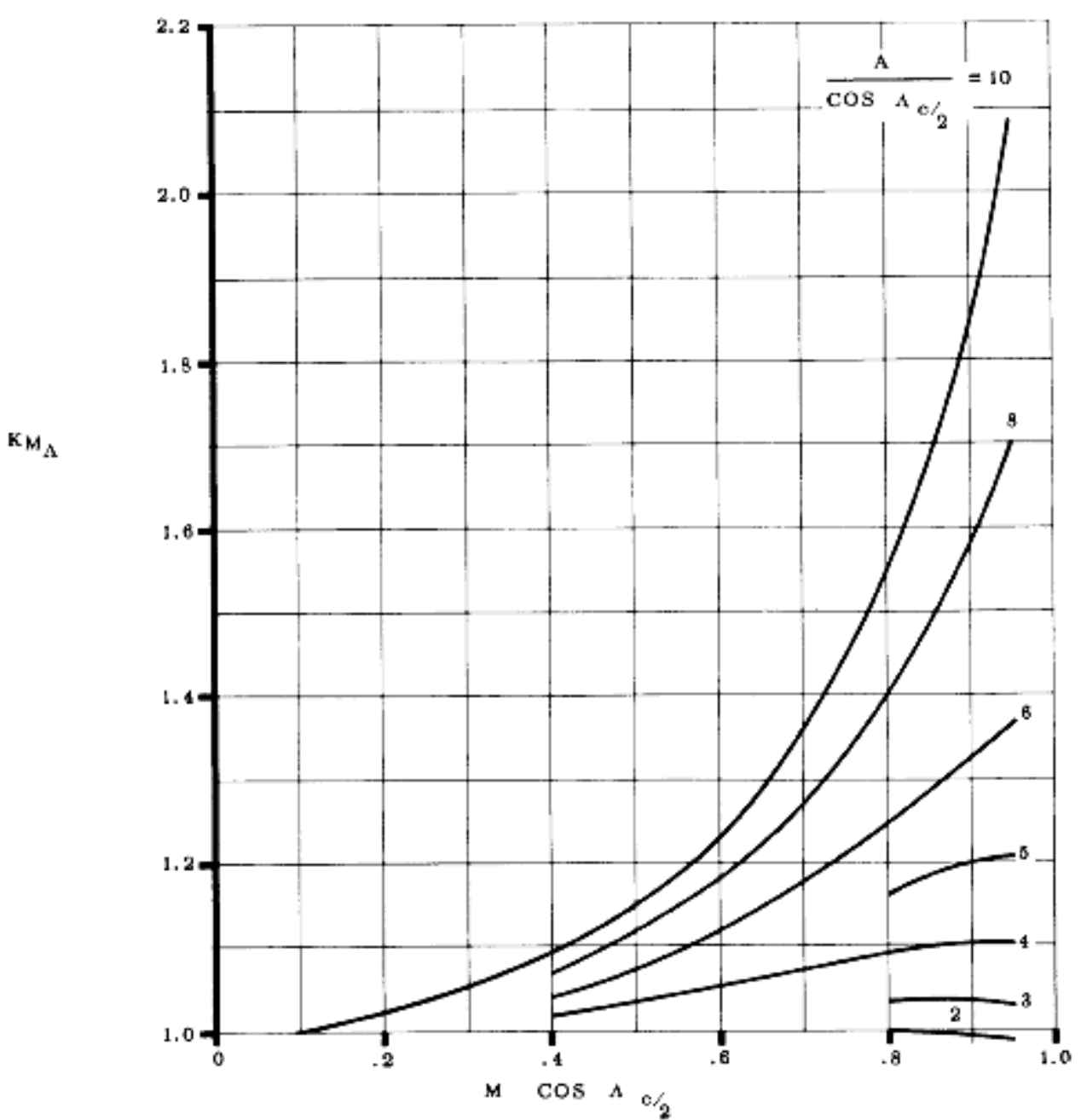

Figure 122: Compressibility Correction Factor to Wing Sweep [Reproduced from Reference 42] 


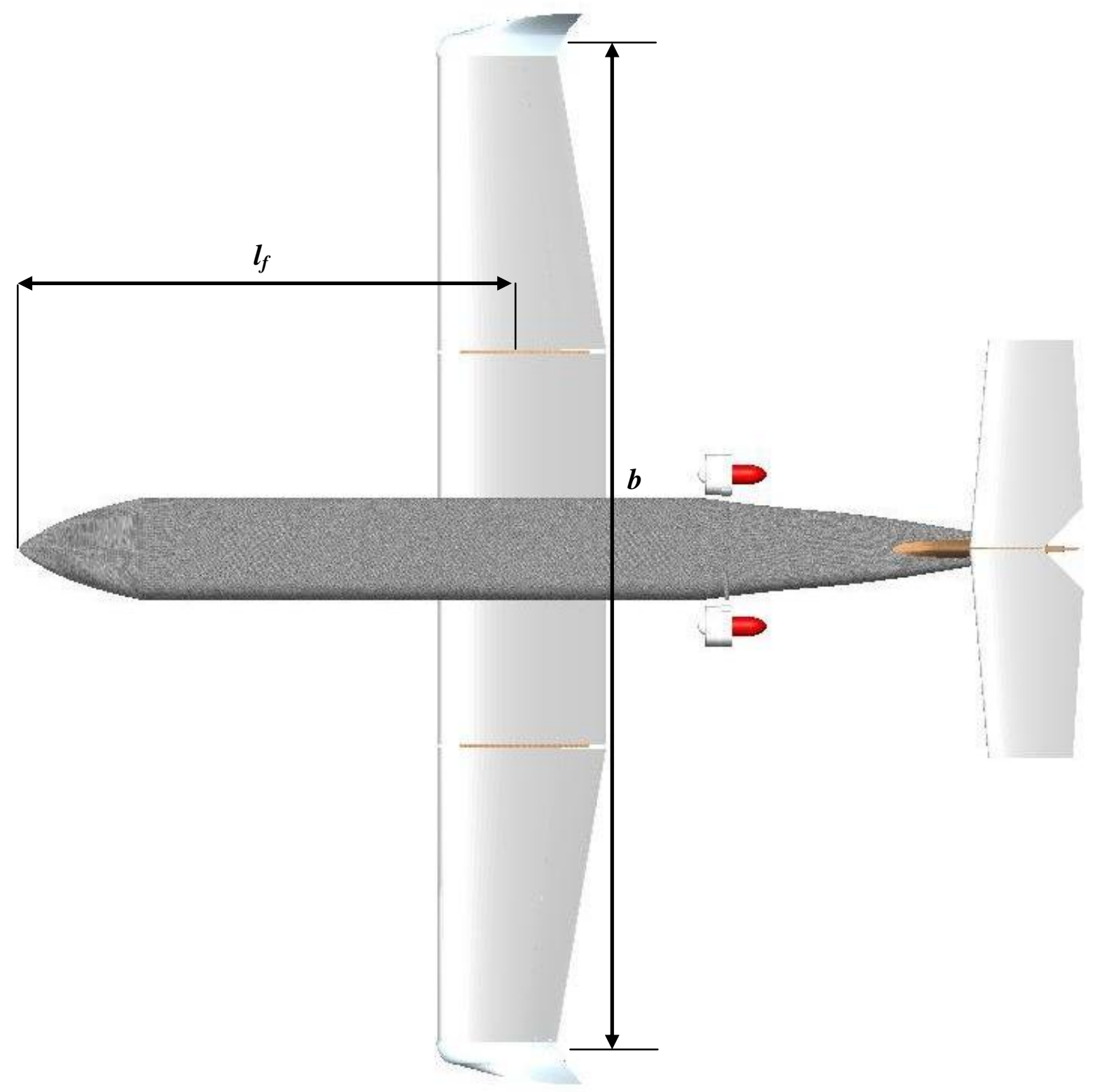

Figure 123: Fuselage and Wing Geometric Parameters 


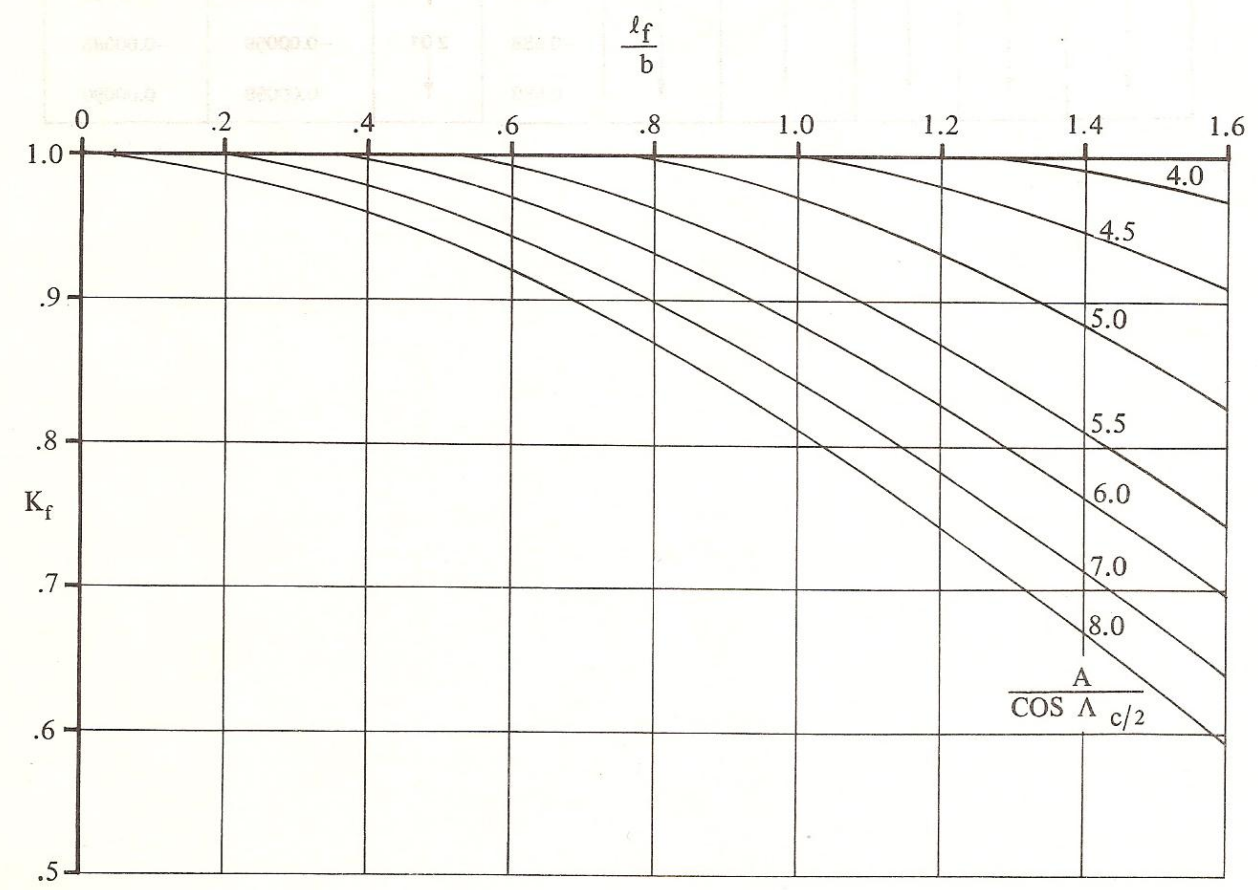

Figure 124: Fuselage Correction Factor [Reproduced from Reference 42]

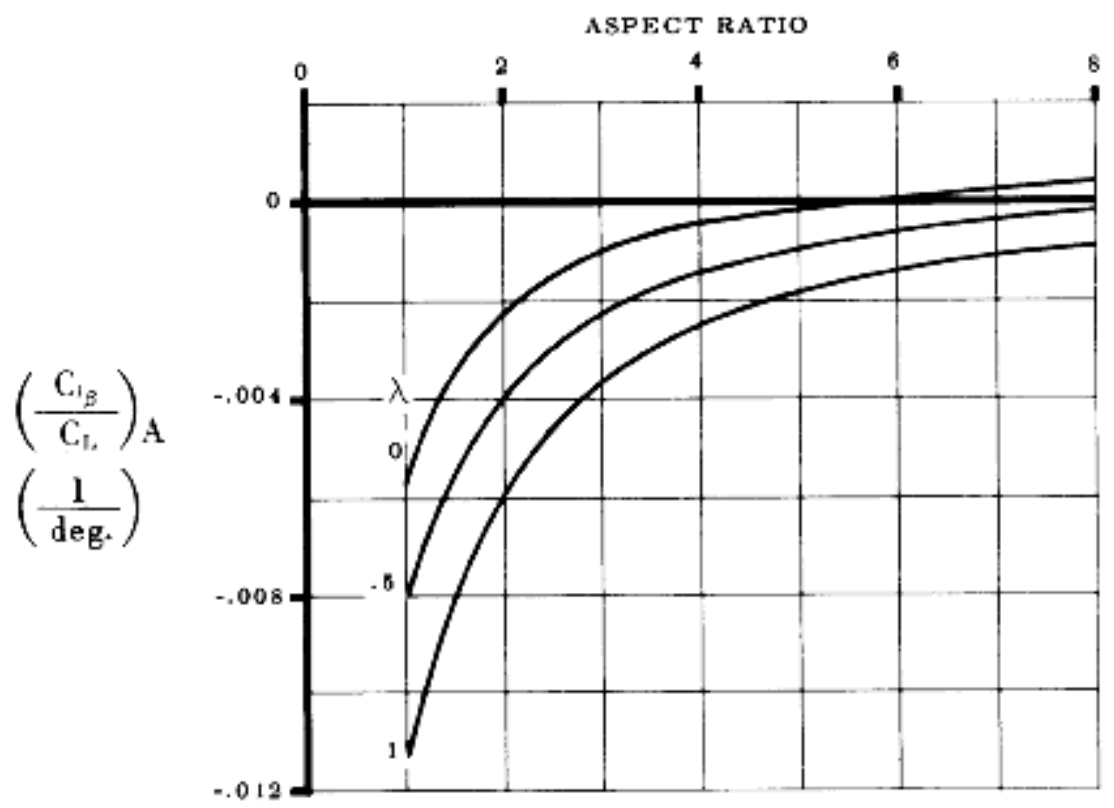

Figure 125: Aspect Ratio Contribution to Rolling Moment due to Sideslip [Reproduced from Reference 42] 


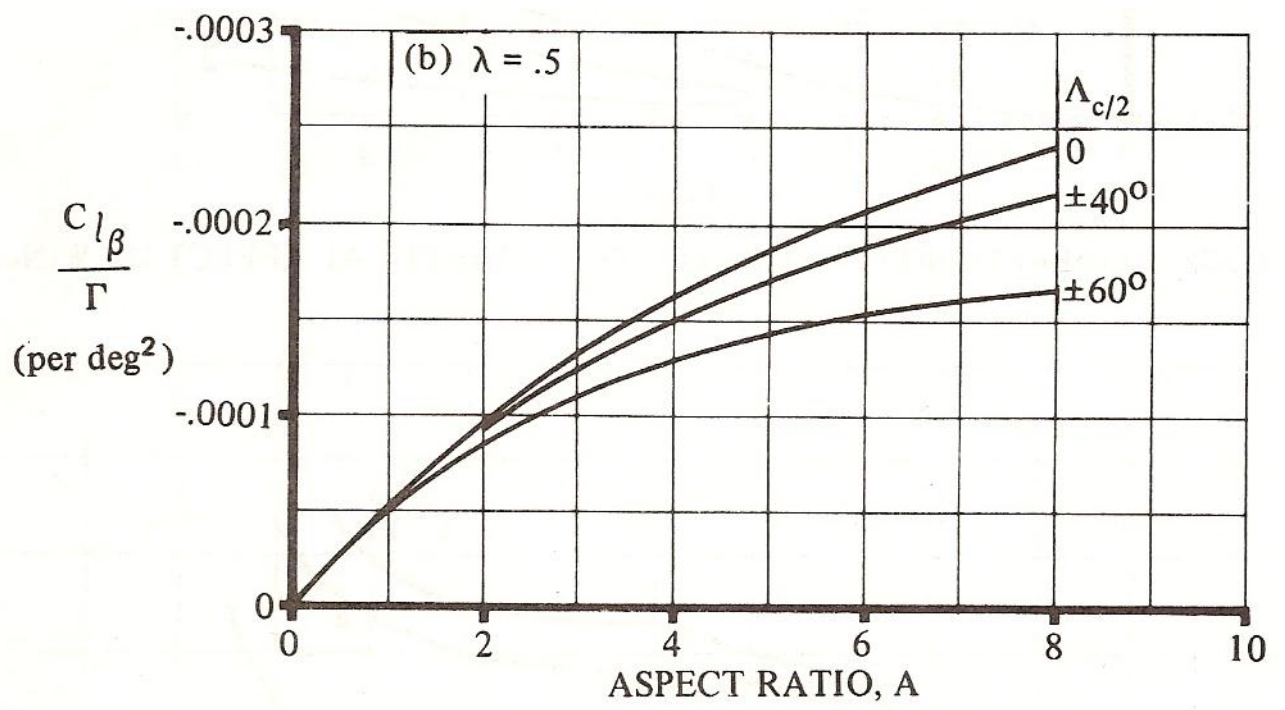

Figure 126: Wing Geometric Dihedral Contribution to Rolling Moment due to Sideslip [Reproduced from Reference 42]

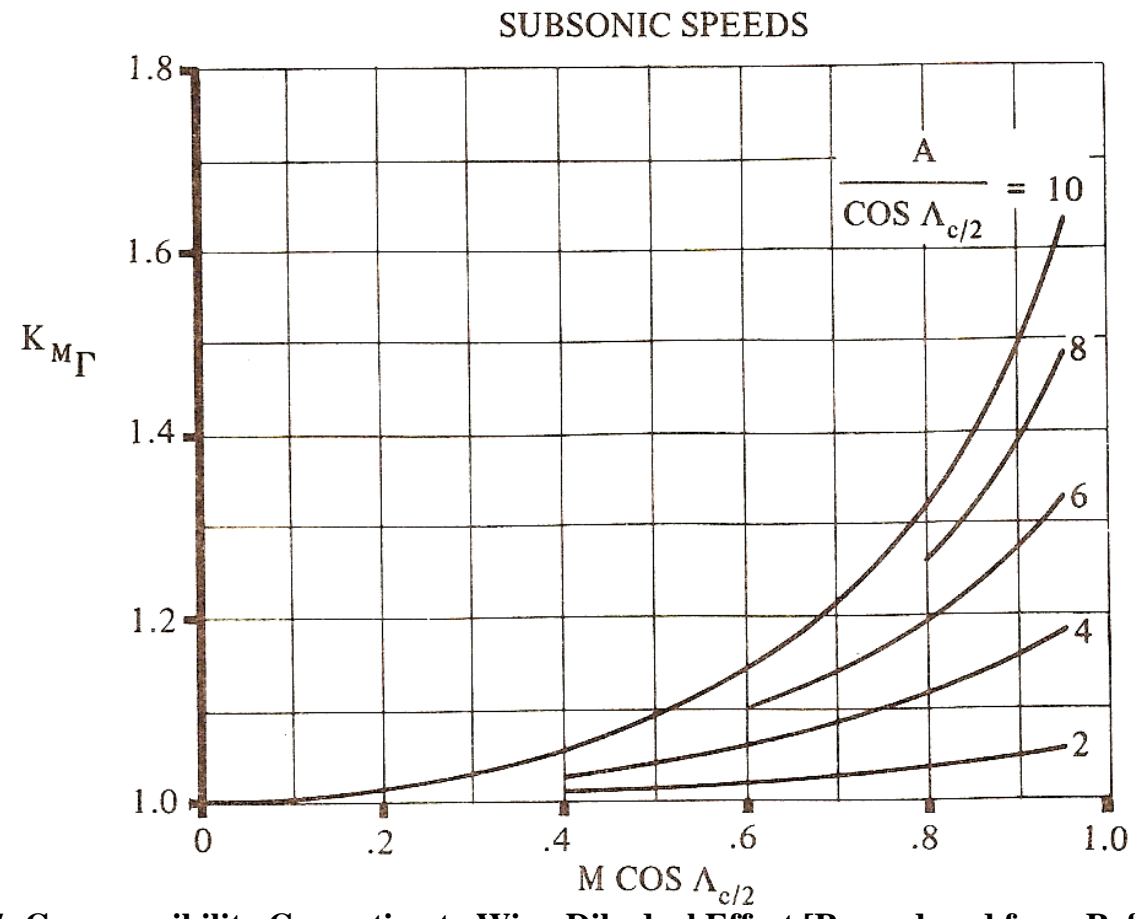

Figure 127: Compressibility Correction to Wing Dihedral Effect [Reproduced from Reference 42] 


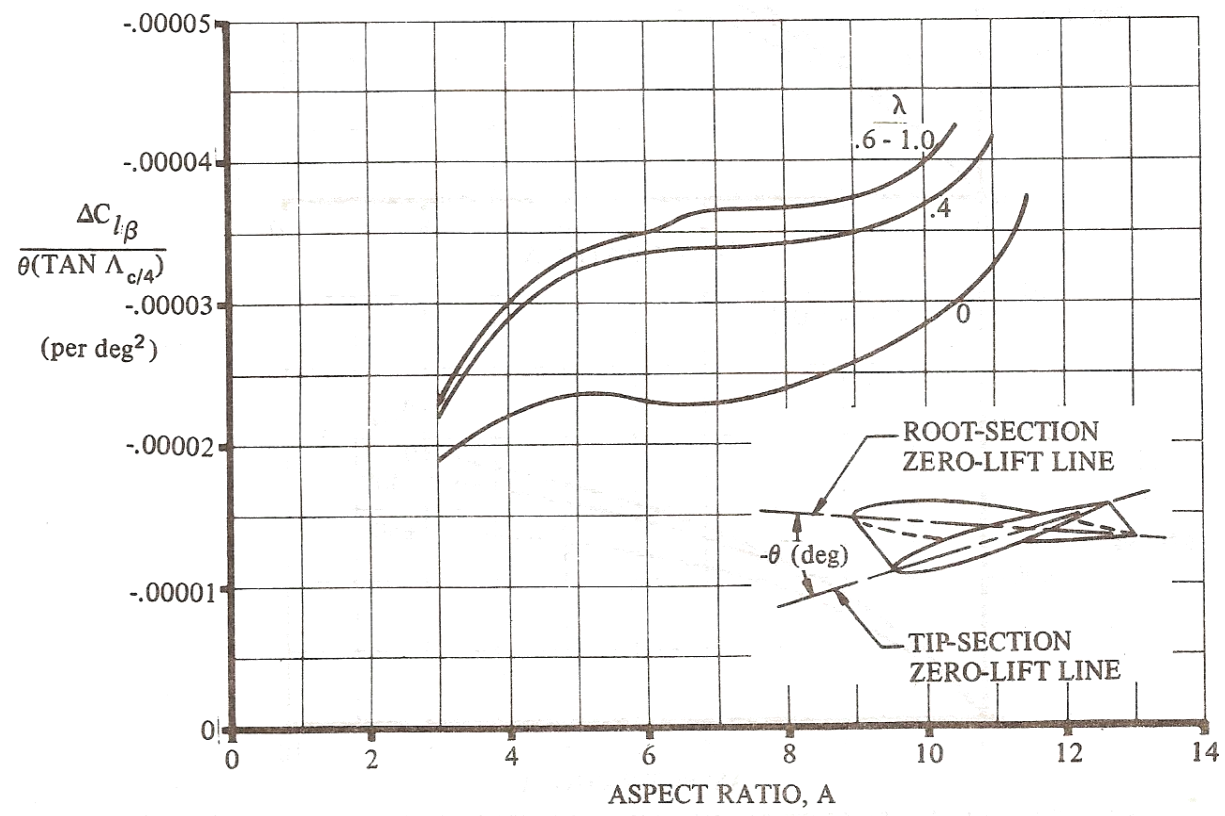

Figure 128: Effect of Wing Twist on Rolling Moment due to Sideslip [Reproduced from Reference 42]

The horizontal tail contribution was estimated using Equation 7.98.

$$
c_{l_{\beta_{H}}}=\left(c_{l_{\beta_{h f}}}\right)\left(\frac{S_{H} b_{H}}{S b}\right)
$$

where $\left(c_{l_{\beta_{h f}}}\right)$ is the horizontal tail dihedral effect calculated using Equation 7.94 and using the appropriate horizontal tail parameters.

The vertical tail contribution was estimated using Equation 7.99.

$$
c_{l_{\beta_{v}}}=\left(c_{Y_{\beta_{v}}}\right)\left(\frac{z_{v} \cos \alpha-l_{v} \sin \alpha}{b}\right)
$$

where $z_{v}, l_{v}$, and $\alpha$ are defined in Figure 120 .

\subsubsection{Rolling Moment-Due-to-Roll-Rate Derivative: $c_{l_{p}}$}

The rolling-moment-due-to-roll-rate derivative was estimated using the wing, horizontal tail, and vertical tail contributions as shown in Equation 7.100.

$$
c_{l_{p}}=c_{l_{p_{w}}}+c_{l_{p_{H}}}+c_{l_{p_{v}}}
$$

The wing contribution was determined using Equation 7.101. 


$$
c_{l_{p_{w}}}=\left(\frac{\beta c_{l_{p}}}{k}\right)_{c_{L}=0}\left(\frac{k}{\beta}\right)\left(\frac{\left(c_{L_{\alpha_{w}}}\right)_{c_{L}}}{\left(c_{L_{\alpha_{w}}}\right)_{c_{L}=0}}\right)\left(\frac{\left(c_{l_{p}}\right)_{\Gamma}}{\left(c_{l_{p}}\right)_{\Gamma=0}}\right)+\left(\Delta c_{l_{p}}\right)_{d r a g}
$$

where $\left(\frac{\beta c_{l_{p}}}{k}\right)_{c_{L}=0}$ is the roll damping parameter at zero lift found from Figure 129, where $k$ is found from Equation 7.102, $\beta$ is a function of Mach number, $\left(c_{L_{\alpha_{w}}}\right)_{c_{L}=0}$ is the wing lift-curve slope at zero lift, and $\left(c_{L_{\alpha_{w}}}\right)_{c_{L}}$ is the wing lift-curve slope at any lift coefficient and is found as the local slope of the wing $c_{L}$ versus $\alpha$ curve $^{42} \cdot\left(\frac{\left(c_{l_{p}}\right)_{\Gamma}}{\left(c_{l_{p}}\right)_{\Gamma=0}}\right)$ is the dihedral effect parameter as determined by Equation 7.103.

(c) $\lambda=0.50$

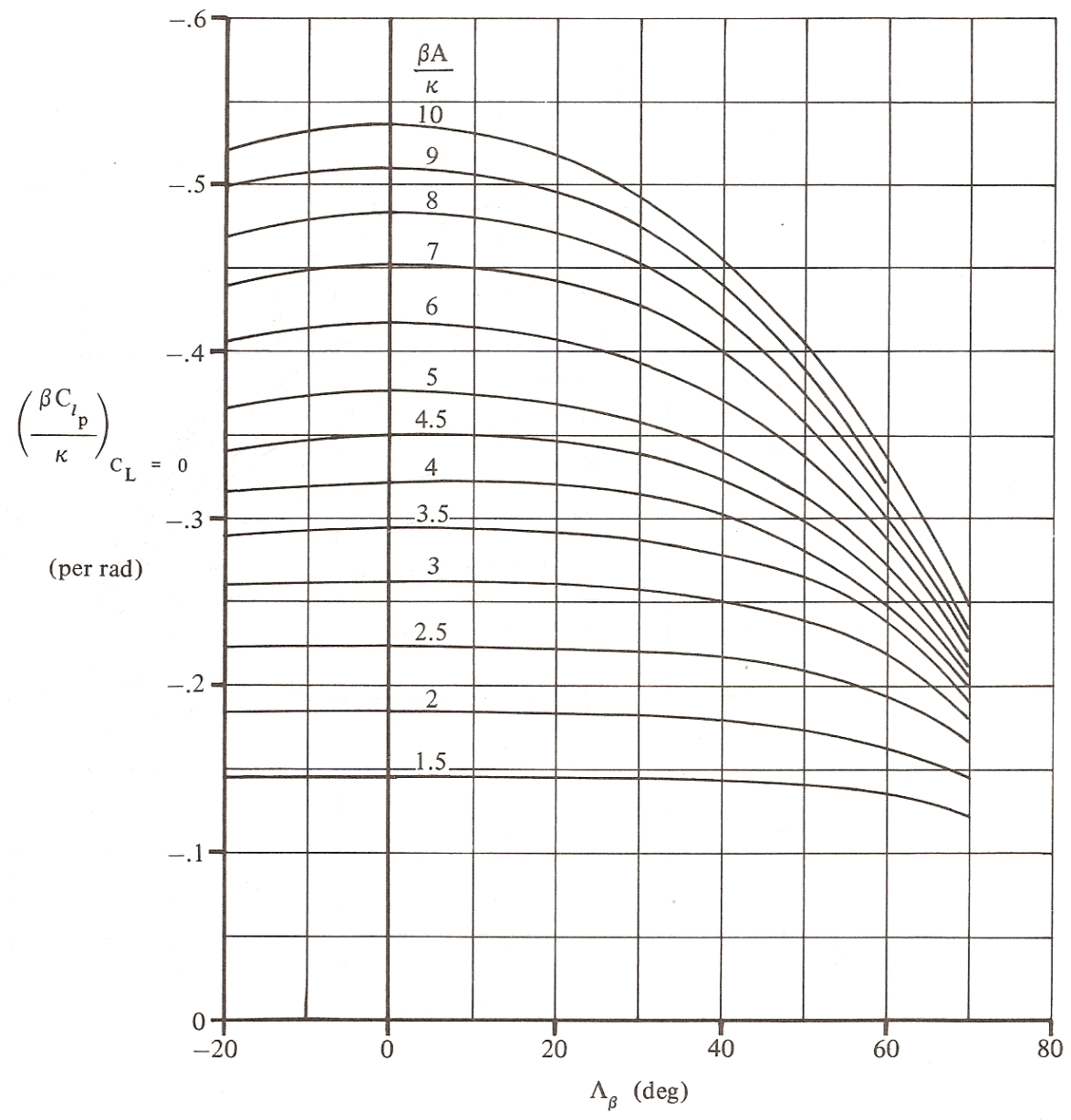

Figure 129: Roll Damping Parameter at Zero Lift [Reproduced from Reference 42] 


$$
\begin{gathered}
k=\left(c_{l_{\alpha}}\right)_{M}\left(\frac{\beta}{2 \pi}\right) \\
\left(\frac{\left(c_{l_{p}}\right)_{\Gamma}}{\left(c_{l_{p}}\right)_{\Gamma=0}}\right)=\left(1-\left(\frac{4 z_{w}}{b}\right) \sin \Gamma+12\left(\frac{z_{w}}{b}\right)^{2}\left(\sin ^{2} \Gamma\right)\right)
\end{gathered}
$$

where $\left(\Delta c_{l_{p}}\right)_{d r a g}$ is the wing drag contribution to roll damping as found from Equation 7.104. For the PACT, the dihedral angle $\Gamma$ is zero.

$$
\left(\Delta c_{l_{p}}\right)_{\text {drag }}=\left(\frac{\left(c_{l_{p}}\right)_{{D_{L}}_{L}}}{\left(c_{L_{w}}\right)^{2}}\right)\left(c_{L_{w}}\right)^{2}-0.125 c_{D_{0_{w}}}
$$

where $\left(\frac{\left(c_{l_{p}}\right)_{c_{D_{L}}}}{\left(c_{L_{w}}\right)^{2}}\right)$ is the drag-due-to-lift roll damping parameter as found from Figure 130.

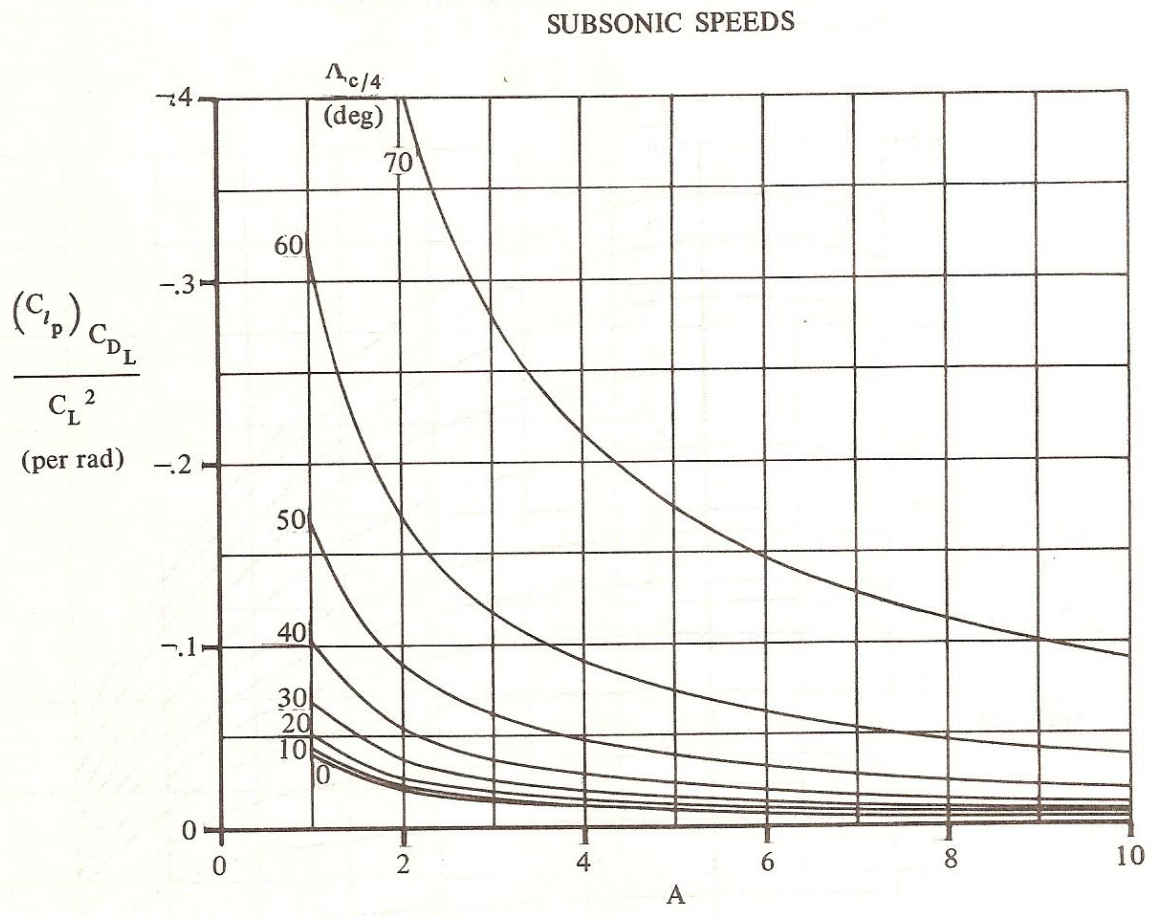

Figure 130: Drag-due-to-Lift Roll-Damping Parameter [Reproduced from Reference 42]

The fuselage contribution to the roll-moment-due-to-roll-rate derivative is negligible for aircraft with $\frac{d_{f}}{b}<0.3$, of which the PACT falls into that category. The horizontal tail 
contribution was estimated from Equation 7.105, where the subscript $H$ represents the respective horizontal tail parameters.

$$
c_{l_{p_{H}}}=0.5\left(c_{l_{p}}\right)_{H}\left(\frac{S_{H}}{S}\right)\left(\frac{b_{H}}{b}\right)^{2}
$$

The vertical tail contribution is estimated using Equation 7.106, where $z_{v}$ is defined from Figure 120 and $c_{Y_{\beta_{v}}}$ is determined using Equation 7.87.

$$
c_{l_{p_{v}}}=2\left(\frac{z_{v}}{b}\right)^{2} c_{Y_{\beta_{v}}}
$$

\subsubsection{Rolling-Moment-Due-to-Yaw-Rate Derivative: $c_{l_{r}}$}

The rolling-moment-due-to-yaw-rate derivative was estimated using Equation 7.107, which represents the wing and vertical tail contributions.

$$
c_{l_{r}}=c_{l_{r_{w}}}+c_{l_{r_{v}}}
$$

The wing contribution was estimated using Equation 7.108, where the geometric dihedral angle for the PACT is zero.

$$
c_{l_{r_{w}}}=\left(c_{L_{w}}\right)\left(\frac{c_{l_{r}}}{c_{L}}\right)_{\substack{c_{L}=0 \\ M}}+\left(\frac{\Delta c_{l_{r}}}{\Gamma}\right) \Gamma+\left(\frac{\Delta c_{l_{r}}}{\varepsilon_{t}}\right) \varepsilon_{t}+\left(\frac{\Delta c_{l_{r}}}{\alpha_{\delta_{f}} \delta_{f}}\right)\left(\alpha_{\delta_{f}}\right) \delta_{f}
$$

where $\left(\frac{c_{l_{r}}}{c_{L}}\right)_{\substack{C_{L}=0 \\ M=0}}$ is the slope of the low-speed rolling moment due to yaw rate at zero lift as found from Figure 131, $\left(\frac{\Delta c_{l_{r}}}{\varepsilon_{t}}\right)$ is the increment in $c_{l_{r}}$ due to twist found from Figure 132, and $\left(\frac{\Delta c_{l_{r}}}{\alpha_{\delta_{f}} \delta_{f}}\right)$ is the effect of symmetric flap deflection on the rolling moment due to roll rate as found from Figure 133. The remaining parameters in Equation 7.108 are defined as follows: 


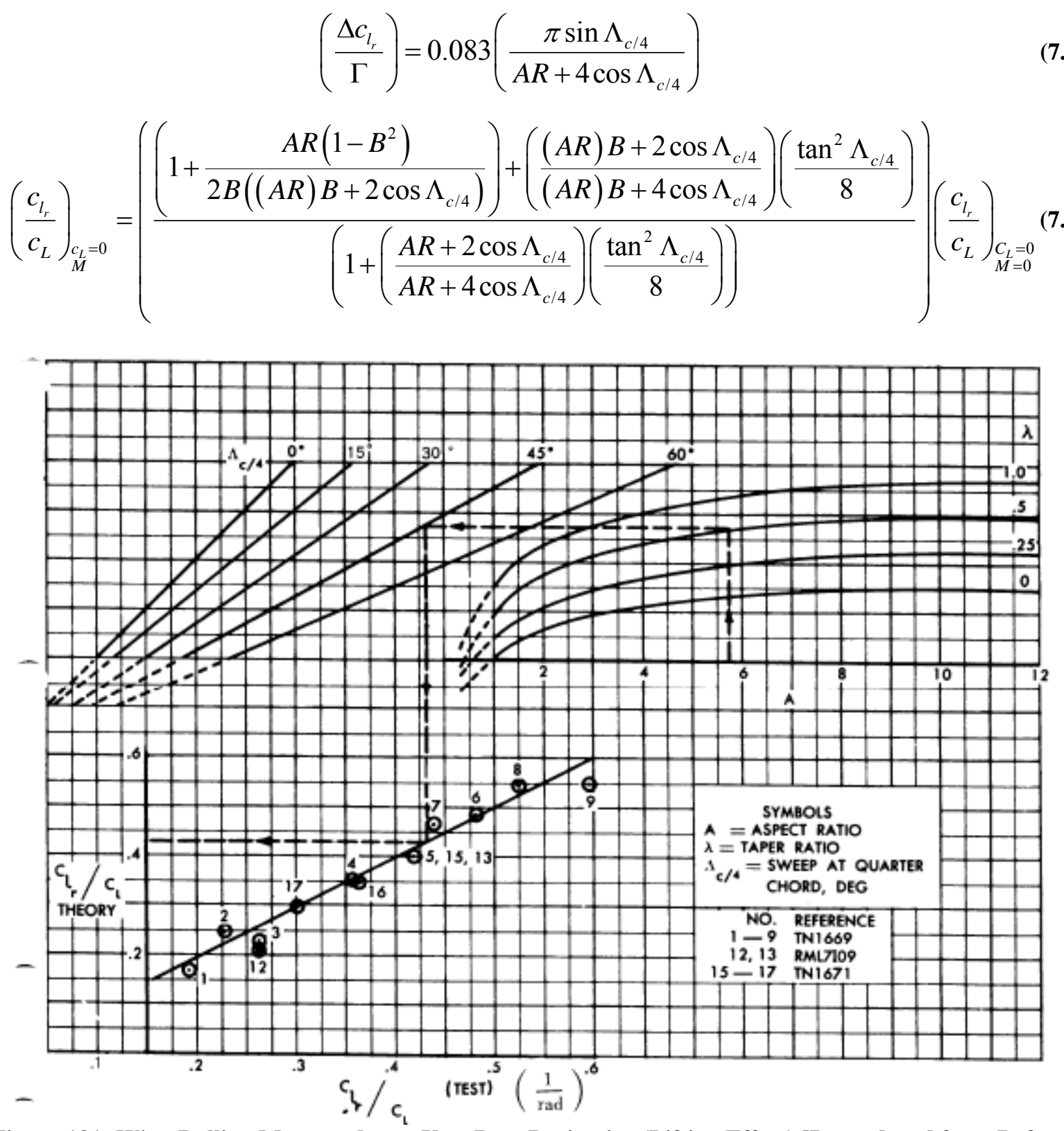

Figure 131: Wing Rolling Moment due to Yaw Rate Derivative (Lifting Effect) [Reproduced from Reference 42] 


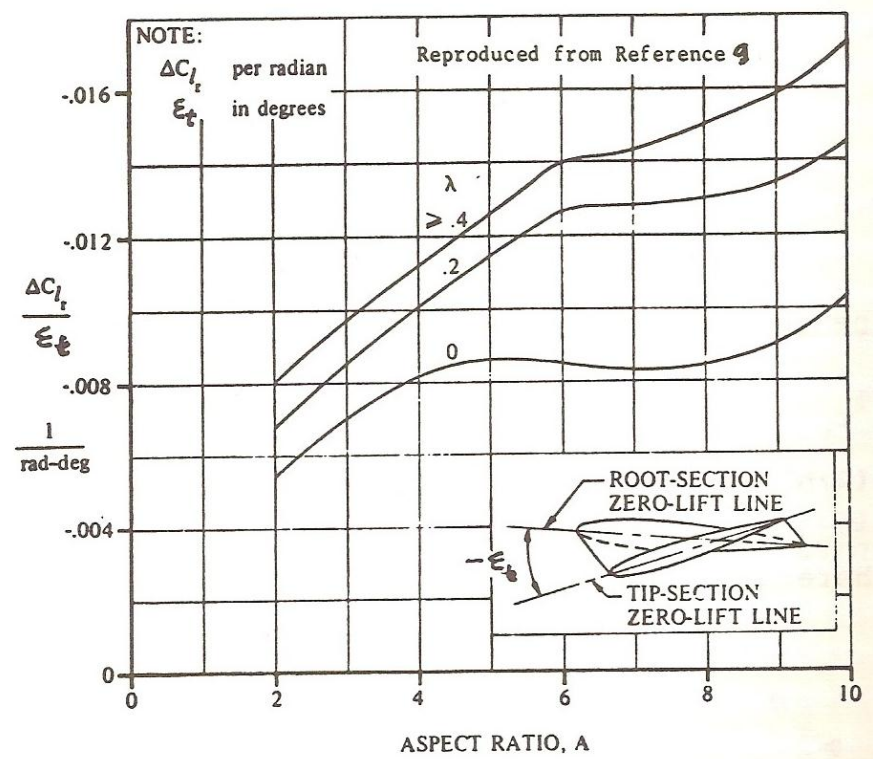

Figure 132: Effect of Wing Twist [Reproduced from Reference 42]

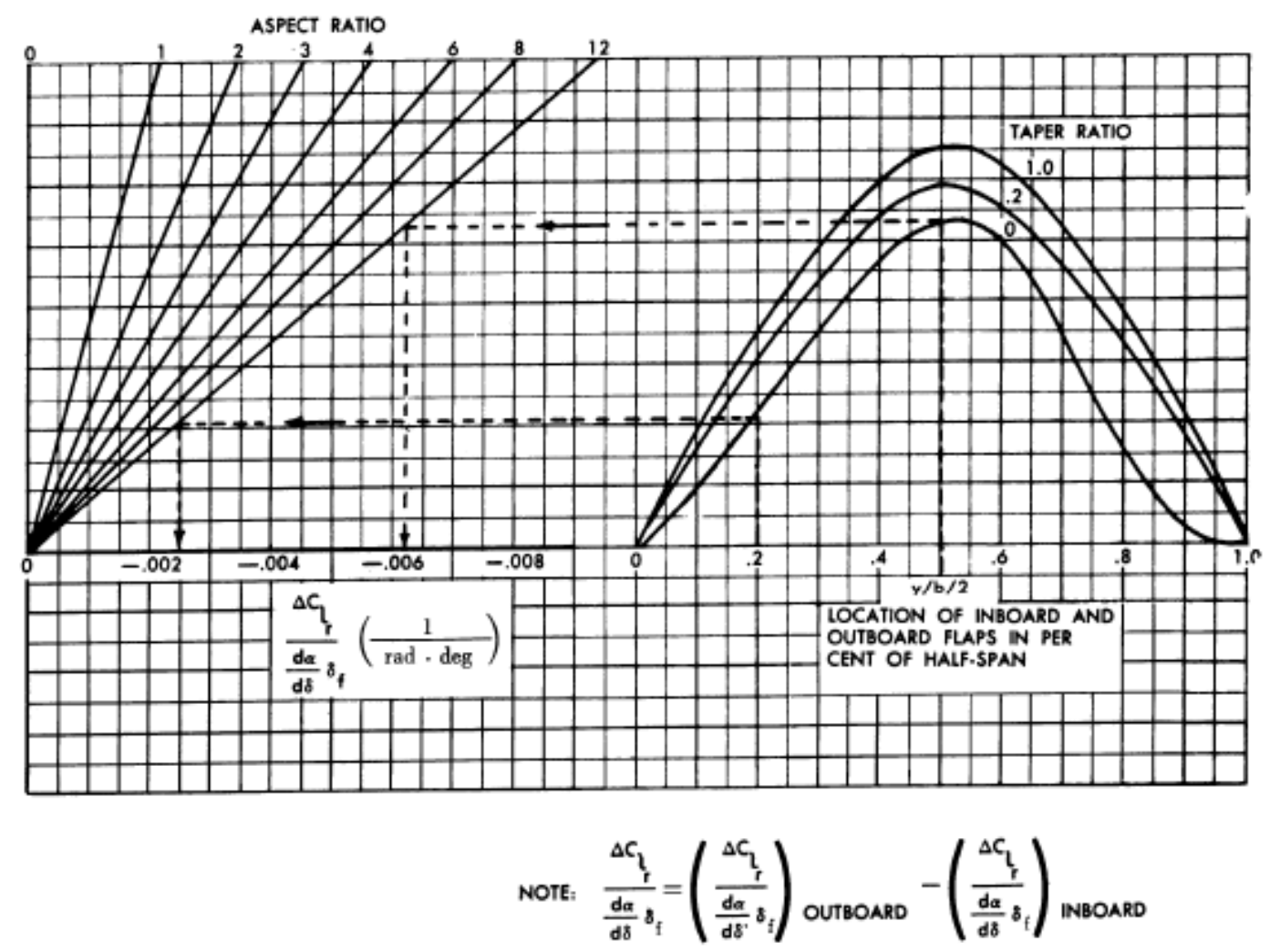

Figure 133: Effect of Symmetric Flap Deflection on $c_{l_{r}}$ [Reproduced from Reference 42]

The vertical tail contribution was estimated using Equation 7.111, where $z_{v}, l_{v}$, and $\alpha$ are defined in Figure 120. 


$$
c_{l_{v}}=-\left(\frac{2}{b^{2}}\right)\left(l_{v} \cos \alpha+z_{v} \sin \alpha\right)\left(z_{v} \cos \alpha-l_{v} \sin \alpha\right) c_{Y_{\beta_{v}}}
$$

\subsubsection{Yawing-Moment-Due-to-Sideslip Derivative: $c_{n_{\beta}}$}

The yawing-moment-due-to-sideslip (static directional stability) derivative was estimated using the wing, fuselage, and vertical tail contributions as shown in Equation 7.112.

$$
c_{n_{\beta}}=c_{n_{\beta_{w}}}+c_{n_{\beta_{f}}}+c_{n_{\beta_{v}}}
$$

The wing contribution is only important at high angles of attack so for steady level flight regimes, it can be estimated as $c_{n_{\beta_{w}}}=0$. The fuselage contribution was found using Equation 7.113, using geometric parameters defined in Figures 134 and 135.

$$
c_{n_{\beta_{f}}}=-57.3 K_{N} K_{R_{l}}\left(\frac{S_{f_{s}} l_{f}}{S b}\right)
$$

where $K_{N}$ is an empirical factor found from Figure 134 and $K_{R_{l}}$ is a factor dependent on Reynolds Number found from Figure 135. 


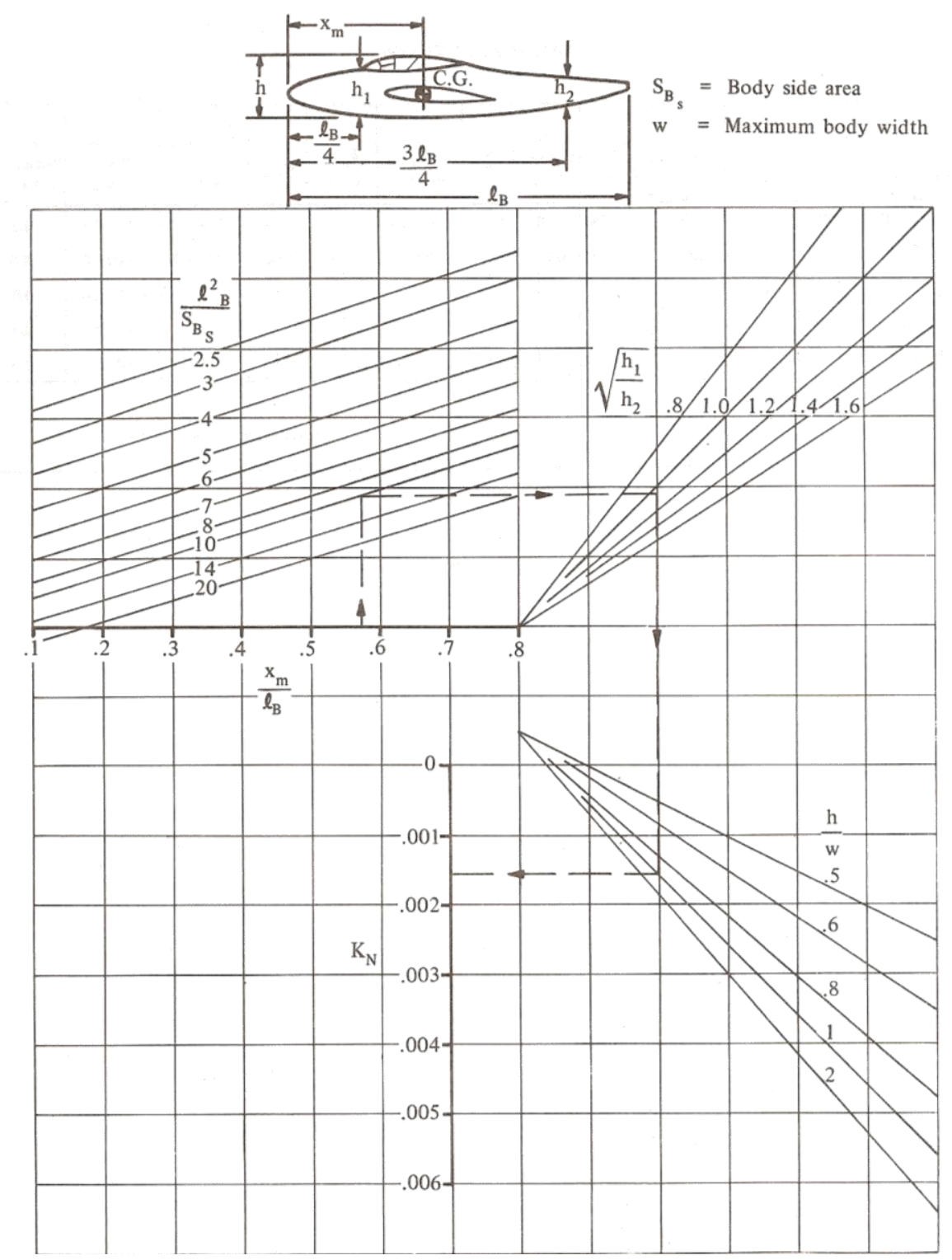

Figure 134: Empirical Factor $K_{N}$ Accounting for Wing-Body Interference with Directional Stability [Reproduced from Reference 42] 


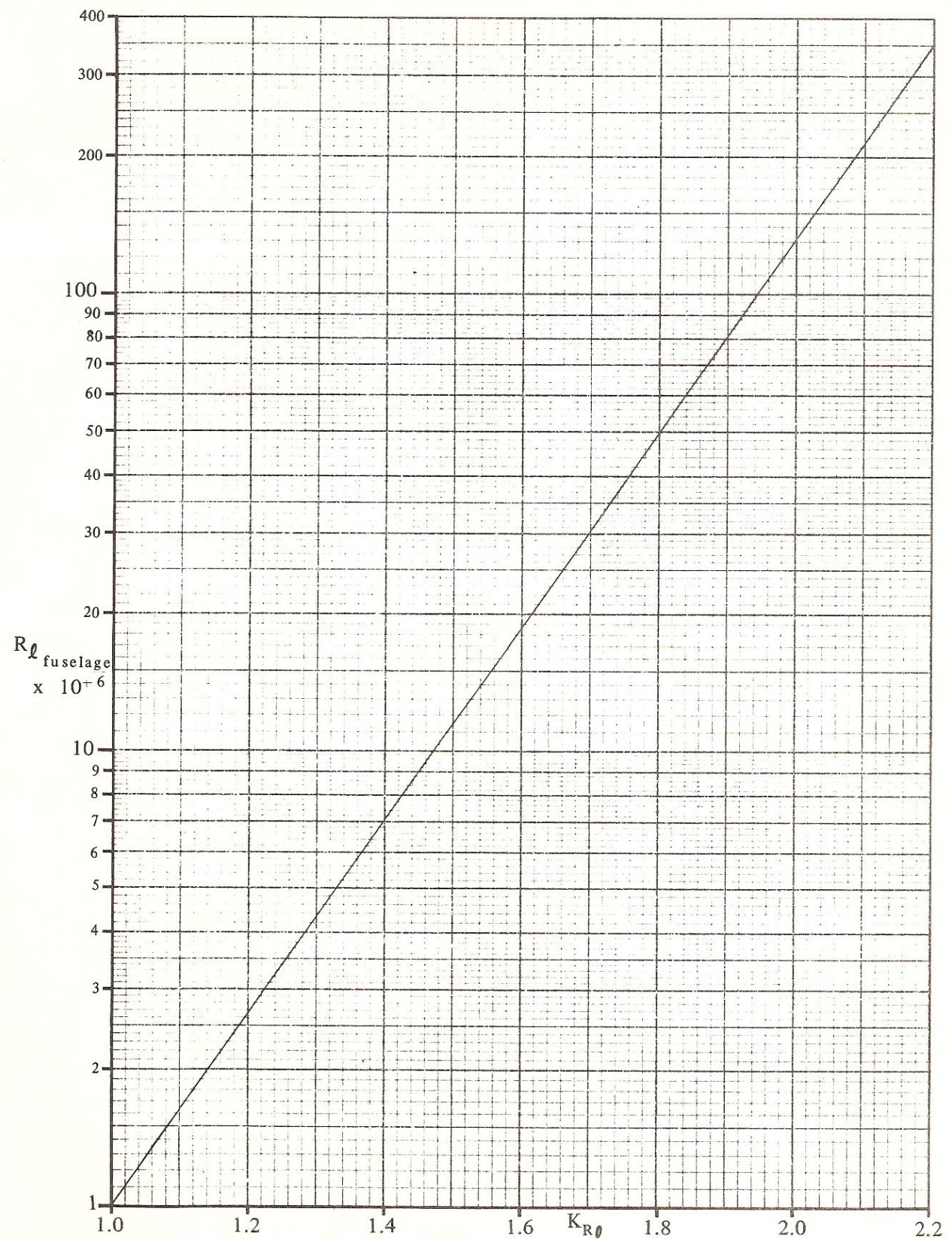

Figure 135: Effect of Fuselage Reynold's Number on Wing-Body Directional Stability [Reproduced from Reference 42]

The horizontal tail contribution was estimated using Equation 7.114, where $l_{v}, z_{v}$, and $\alpha$ are defined from Figure 120. 


$$
c_{n_{\beta_{v}}}=-\left(c_{Y_{\beta_{v}}}\right)\left(\frac{l_{v} \cos \alpha-z_{v} \sin \alpha}{b}\right)
$$

\subsubsection{Yawing-Moment-Due-to-Roll-Rate Derivative: $c_{n_{p}}$}

The yawing-moment-due-to-roll-rate derivative was estimated using the wing and vertical tail contributions as shown in Equation 7.115.

$$
c_{n_{p}}=c_{n_{p_{w}}}+c_{n_{p_{v}}}
$$

The wing contribution to this derivative was estimated using Equation 7.116.

$$
c_{n_{p_{w}}}=-\left(\frac{c_{n_{p}}}{c_{L}}\right)_{c_{L}=0} c_{L}+\left(\frac{c_{n_{p}}}{\varepsilon_{t}}\right) \varepsilon_{t}+\left(\frac{\Delta c_{n_{p}}}{\left(\alpha_{\delta_{f}}\right)\left(\delta_{f}\right)}\right)\left(\alpha_{\delta_{f}}\right) \delta_{f}
$$

where $\left(\frac{c_{n_{p}}}{\varepsilon_{t}}\right)$ is the wing twist contribution found from Figure 136, $\left(\frac{\Delta c_{n_{p}}}{\left(\alpha_{\delta_{f}}\right)\left(\delta_{f}\right)}\right)$ is the contribution due to symmetrical flap deflection as found from Figure 137, and the remaining parameters are defined as follows:

$$
\begin{gathered}
\left.\left(\frac{c_{n_{p}}}{c_{L}}\right)_{c_{L}=0}=\left(\frac{A R+4 \cos \Lambda_{c / 4}}{(A R) B+4 \cos \Lambda_{c / 4}}\right)\left(\frac{(A R) B+0.5\left((A R) B+\cos \Lambda_{c / 4}\right) \tan ^{2} \Lambda_{c / 4}}{A R+0.5\left(A R+\cos \Lambda_{c / 4}\right) \tan ^{2} \Lambda_{c / 4}}\right)\left(\frac{c_{n_{p}}}{c_{L}}\right)_{\substack{c_{L}=0 \\
M=0}}\right) \\
\left(\frac{c_{n_{p}}}{c_{L}}\right)_{\substack{c_{L}=0 \\
M=0}}=-\frac{1}{6}\left(\frac{A R+6\left(A R+\cos \Lambda_{c / 4}\right)\left(\frac{\bar{x}}{\bar{c}} \frac{\tan \Lambda_{c / 4}}{A R}+\frac{\tan ^{2} \Lambda_{c / 4}}{12}\right)}{A R+4 \cos \Lambda_{c / 4}}\right) \\
\left(\alpha_{\delta_{f}}\right)=\frac{\Delta c_{l}}{c_{l_{\alpha}} \delta_{f}}
\end{gathered}
$$

with $\Delta c_{l}$ found based on the flap geometry. 


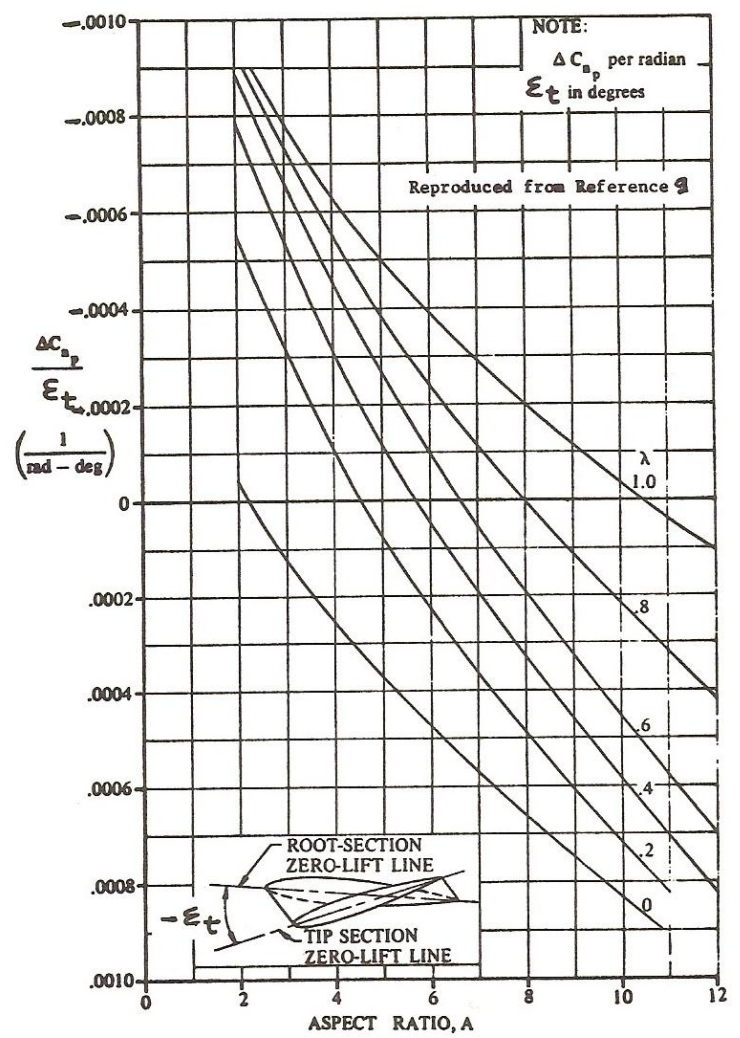

Figure 136: Effect of Wing Twist on Yawing-Moment-due-to-Roll-Rate Derivative [Reproduced from Reference 42] 

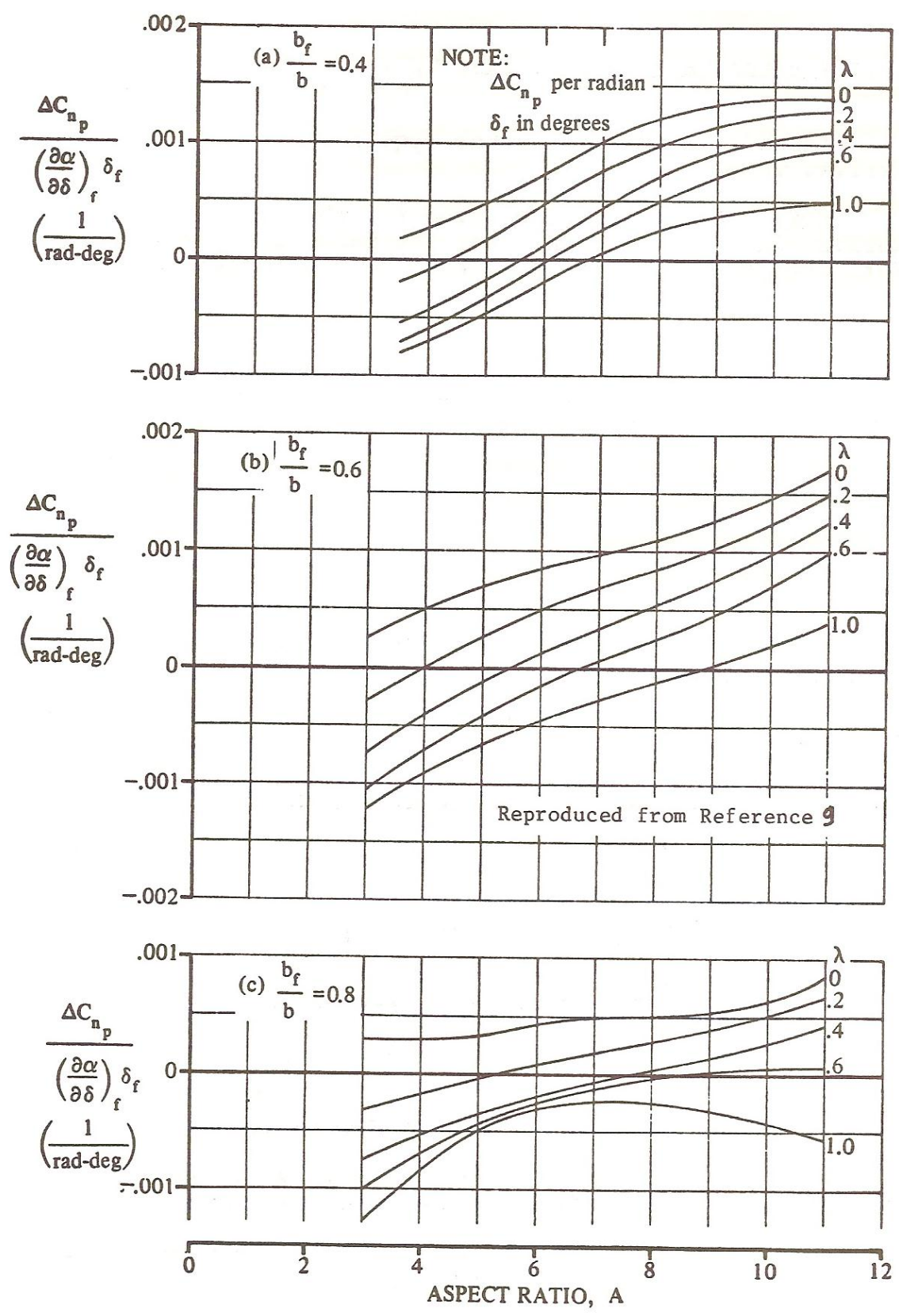

Figure 137: Effect of Symmetrical Flap Deflection on Yawing-Moment-due-to-Roll-Rate Derivative [Reproduced from Reference 42]

The vertical tail contribution was estimated using Equation 7.120, where $z_{v}, l_{v}$, and $\alpha$ are geometric properties defined from Figure 120. 


$$
c_{n_{p_{v}}}=-\left(\frac{2}{b^{2}}\right)\left(l_{v} \cos \alpha+z_{v} \sin \alpha\right)\left(z_{v} \cos \alpha+l_{v} \sin \alpha-z_{v}\right) c_{Y_{\beta_{v}}}
$$

\subsubsection{Yawing Moment-Due-to-Yaw-Rate Derivative: $c_{n_{r}}$}

The yawing-moment-due-to-yaw-rate (yaw-damping) derivative was estimated using the wing and vertical tail contributions as expressed in Equation 7.121.

$$
c_{n_{r}}=c_{n_{r_{w}}}+c_{n_{r_{v}}}
$$

The wing contribution is determined using Equation 7.122, where $\left(\frac{c_{n_{r}}}{c_{L}^{2}}\right)$ is the wing yaw damping derivative lifting effect found from Figure 138, and $\left(\frac{c_{n_{r}}}{c_{D_{0}}}\right)$ is the wing yaw damping derivative drag effect found from Figure 139.

$$
c_{n_{r_{w}}}=\left(\frac{c_{n_{r}}}{c_{L}^{2}}\right)\left(c_{L}\right)^{2}+\left(\frac{c_{n_{r}}}{c_{D_{0}}}\right) c_{D_{0_{w}}}
$$

The horizontal tail contribution was estimated using Equation 7.123 where $z_{v}, l_{v}$, and $\alpha$ are geometric properties defined from Figure 120.

$$
c_{n_{r_{v}}}=\left(\frac{2}{b^{2}}\right)\left(l_{v} \cos \alpha+z_{v} \sin \alpha\right)^{2} C_{Y_{\beta_{v}}}
$$




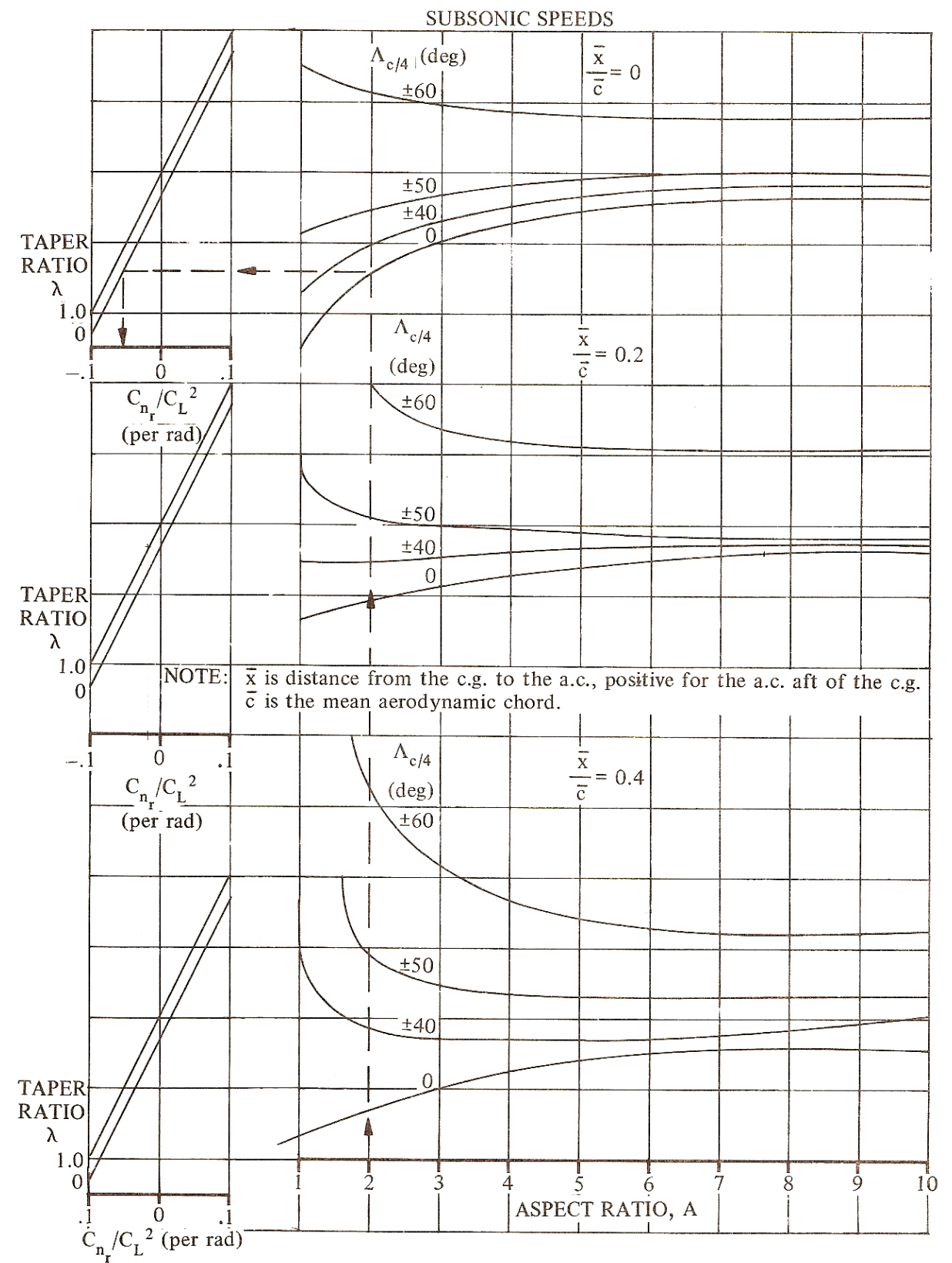

Figure 138: Low-Speed Drag-due-to-Lift Yaw-Damping Parameter [Reproduced from Reference 42] 


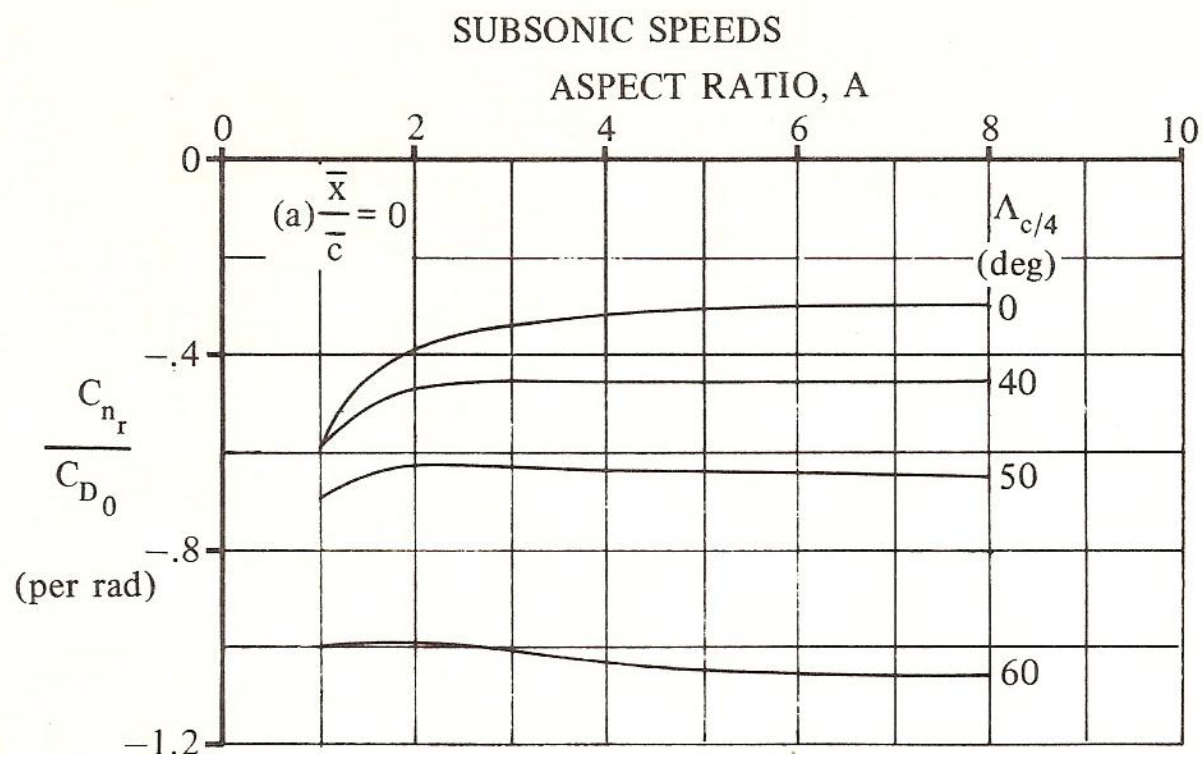

Figure 139: Low-Speed Profile Drag Yaw-Damping Parameter [Reproduced from Reference 42]

\subsubsection{PACT Control Derivatives (DATCOM)}

The control derivatives were calculated for the PACT aircraft for analysis purposes. The following sections detail the methodology used in determining the estimates of the PACT control derivatives.

\subsubsection{Drag-Due-to-Stabilizer-Incidence Derivative: $c_{D_{i H}}$}

The drag-due-to-stabilizer-incidence derivative may be estimated using Equation 7.124.

$$
c_{D_{i_{H}}}=2\left(\frac{c_{L_{H}}}{\pi A R_{H} e_{H}}\right)\left(c_{L_{\alpha_{H}}}\right) \eta_{H}
$$

where $c_{L_{H}}$ is the lift coefficient of the horizontal stabilizer based on the wing area. In this equation, $e_{H}$ represents the Oswald efficiency of the horizontal stabilizer, and is assumed to be 0.75 for T-tail configurations such as the PACT aircraft. Equation 7.125 was used to calculate the lift-curve slope of the horizontal stabilizer. 


$$
c_{L_{\alpha_{H}}}=\frac{2 \pi A R_{H}}{2+\sqrt{\left(\frac{A R_{H}^{2} \beta^{2}}{k^{2}}\right)\left(1+\tan ^{2}\left(\frac{\Lambda_{H c / 2}}{\beta^{2}}\right)+4\right)}}
$$

\subsubsection{Drag-Due-to-Elevator Derivative: $c_{D_{\delta E}}$}

The drag-due-to-elevator derivative was calculated for the PACT aircraft using Equation 7.126. The PACT elevators are full-span control surfaces, that is, they span the entire length of the horizontal stabilizer.

$$
c_{D_{\delta E}}=\left(\alpha_{\delta E}\right) c_{D_{i_{H}}}
$$

where

$$
\alpha_{\delta E}=K_{b}\left\lfloor\frac{c_{l_{\delta}}}{\left(c_{l_{\delta}}\right)_{\text {theory }}}\right\rfloor\left(c_{l_{\delta}}\right)_{\text {theory }}\left(\frac{k^{\prime}}{c_{l_{\alpha_{H}}}}\right)\left[\frac{\left(\alpha_{\delta}\right)_{C_{L}}}{\left(\alpha_{\delta}\right)_{c_{l}}}\right]
$$

where $K_{b}$ is the elevator span factor and is 1.0 for the PACT due to the full span elevator, $\frac{c_{l_{\delta}}}{\left(c_{l_{\delta}}\right)_{\text {theory }}}$ is the correction factor for plain flap lift found from Figure 140, $\left(c_{l_{\delta}}\right)_{\text {theory }}$ is the lift effectiveness of a plain flap as found from Figure 141, $k$ ' is a correction factor for nonlinear lift behavior of plain flaps found from Figure 142, and $\frac{\left(\alpha_{\delta}\right)_{C_{L}}}{\left(\alpha_{\delta}\right)_{c_{l}}}$ is the effect of aspect ratio and flapchord ratio on three dimensional flap effectiveness found from Figure 143. 


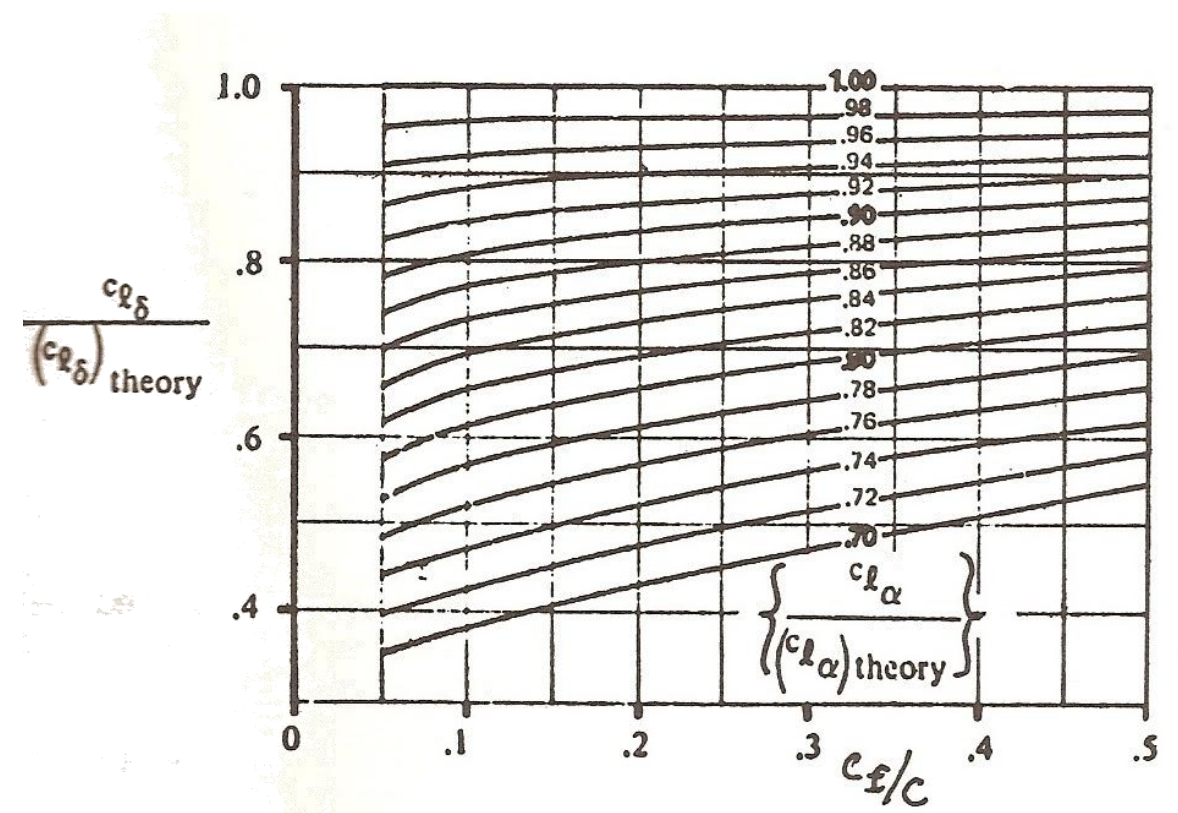

Figure 140: Correction Factor for Plain Flap Lift [Reproduced from Reference 42]

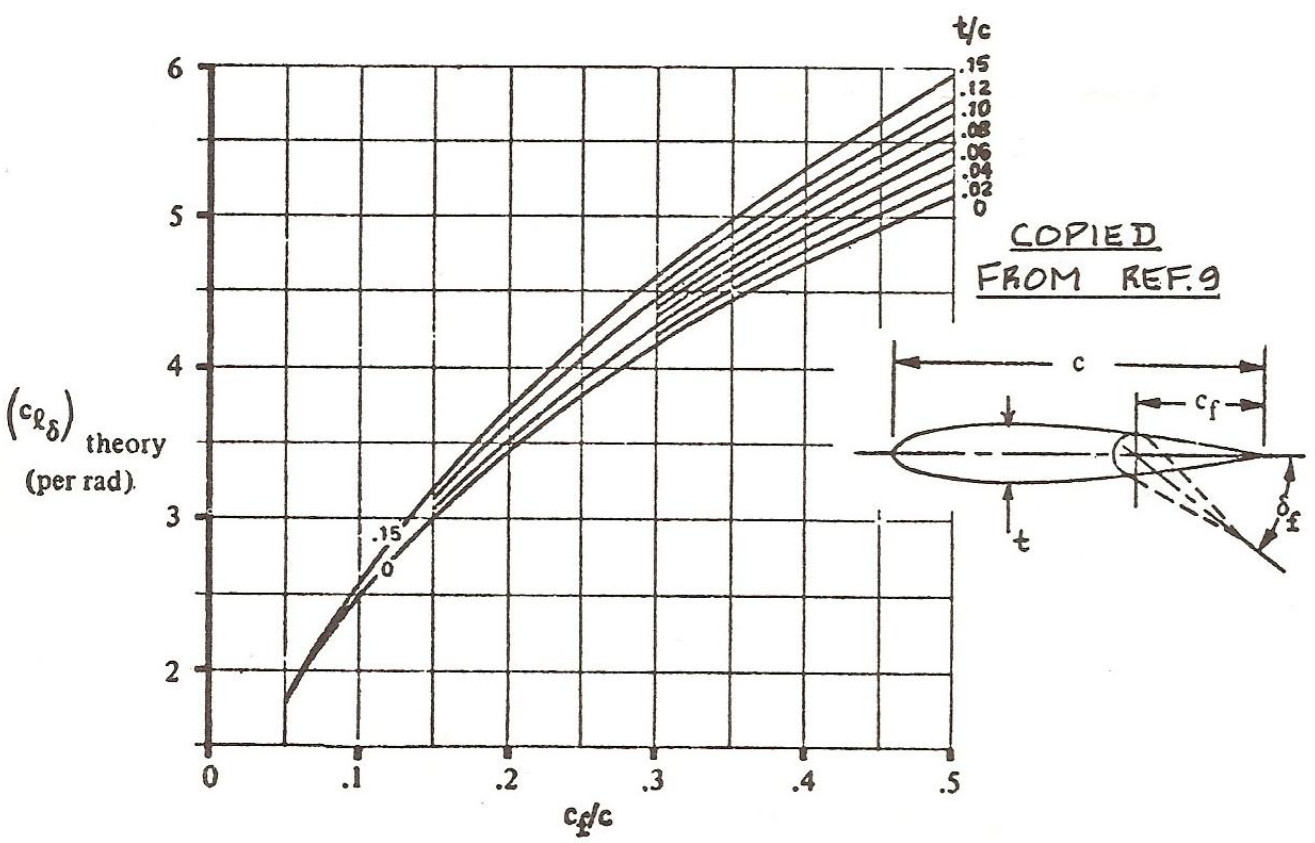

Figure 141: Lift Effectiveness of a Plain Flap [Reproduced from Reference 42] 


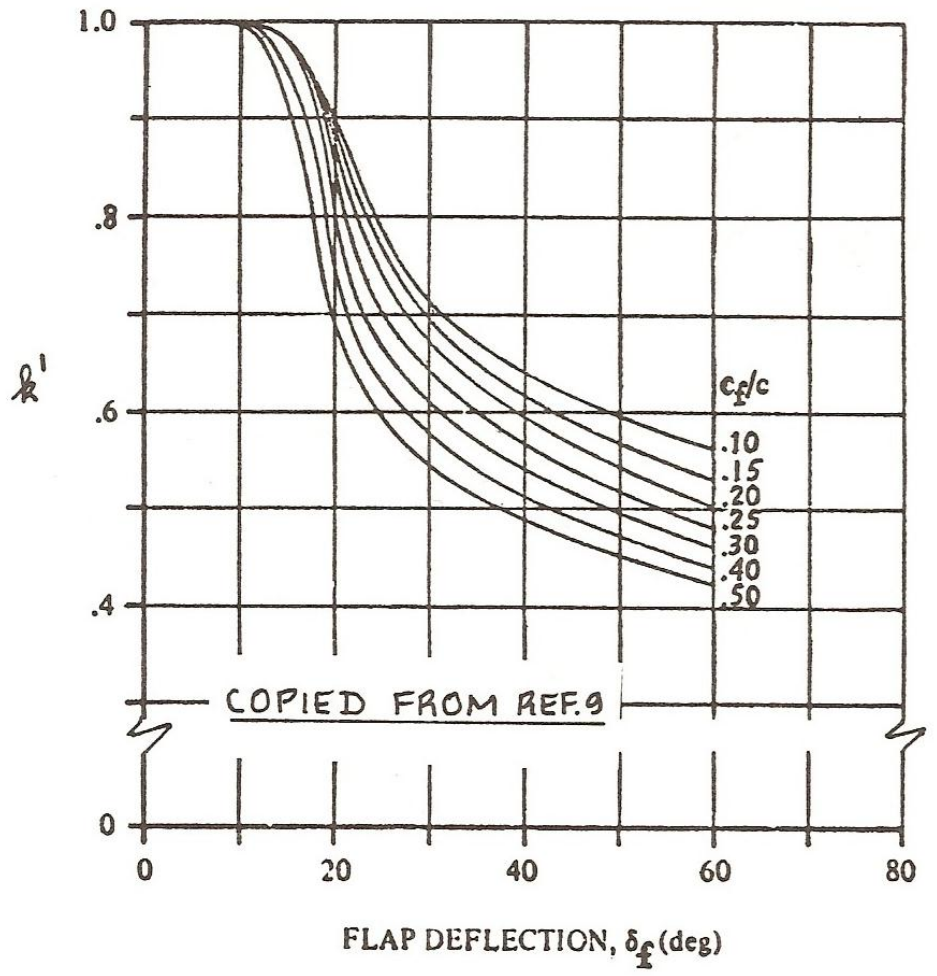

Figure 142: Correction Factor for Nonlinear Lift Behavior of Plain Flaps [Reproduced from Reference 42]

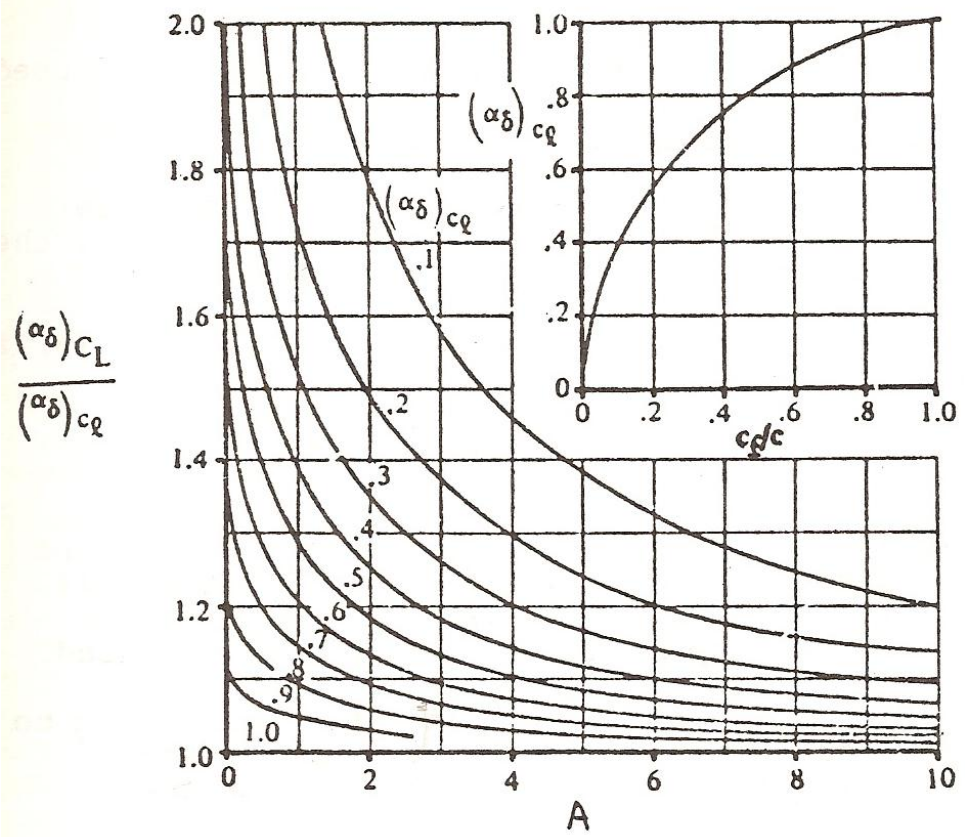

Figure 143: Aspect Ratio and Flap-Chord Ratio Effect on Three-Dimensional Flap Effectiveness [Reproduced from Reference 42] 


\subsubsection{Lift-Due-to-Stabilizer-Incidence Derivative: $c_{L_{H H}}$}

The lift-due-to-stabilizer-incidence derivative was estimated using Equation 7.128, utilizing the geometric properties of the horizontal stabilizer.

$$
c_{L_{i H}}=\eta_{H}\left(\frac{S_{H}}{S}\right) c_{L_{\alpha_{H}}}
$$

\subsubsection{Lift-Due-to-Elevator Derivative: $c_{L_{S E}}$}

The lift-due-to-elevator derivative was estimated using Equation 7.129, where $\alpha_{\delta E}$ was calculated using Equation 7.127

$$
c_{L_{\delta E}}=\left(\alpha_{\delta E}\right) c_{L_{i_{H}}}
$$

7.2.3.5 Pitching-Moment-Due-to-Stabilizer-Incidence Derivative: ${ }^{c_{m_{i H}}}$

The pitching moment-due-to-stabilizer-incidence derivative was estimated using Equation 7.130 .

$$
c_{m_{i H}}=-\left(c_{L_{\alpha_{H}}}\right) \eta_{H}\left(\bar{x}_{a c_{H}}-\bar{x}_{C G}\right)\left(\frac{S_{H}}{S}\right)
$$

\subsubsection{Pitching-Moment-Due-to-Elevator Derivative: $c_{m_{\delta E}}$}

The pitching-moment-due-to-elevator derivative was estimated using Equation 7.131 where $\alpha_{\delta E}$ was calculated using Equation 7.127.

$$
c_{m_{\delta E}}=\left(\alpha_{\delta E}\right) c_{m_{i H}}
$$

\subsubsection{Side-Force-Due-to-Aileron Derivative: $c_{Y_{\delta A}}$}

The side-force-due-to-aileron derivative is negligible for most standard aileron conventions unless ailerons are located in close proximity to the vertical tail ${ }^{42}$. For the PACT aircraft, the ailerons are not located within a close range of the vertical tail so this derivative was estimated to be zero through this analysis.

$$
c_{Y_{\delta A}}=0
$$

\subsubsection{Side-Force-Due-to-Rudder Derivative: $c_{Y_{\delta R}}$}

The side-force-due-to-rudder derivative was estimated using Equation 7.133. 


$$
c_{Y_{\delta R}}=\left(c_{L_{\alpha V}}\right)\left(k^{\prime} K_{b}\right)\left(\frac{\left(\alpha_{\delta}\right)_{C_{L}}}{\left(\alpha_{\delta}\right)_{c_{l}}}\right)\left(\alpha_{\delta}\right)_{c_{l}}\left(\frac{S_{V}}{S}\right)
$$

where $k^{\prime}$ is a correction factor for nonlinear lift behavior of plain flaps, $K_{b}$ is the rudder span factor and is 1.0 for the PACT due to the full span rudder, and $\frac{\left(\alpha_{\delta}\right)_{C_{L}}}{\left(\alpha_{\delta}\right)_{c_{l}}}$ is the effect of aspect ratio and flap-chord ratio on three dimensional flap effectiveness.

\subsubsection{Rolling-Moment-Due-to-Aileron Derivative: $c_{l_{\delta A}}$}

The rolling-moment-due-to-aileron (roll control power) derivative calculation is a multi-step process. The following steps ${ }^{42}$ must be taken to estimate this derivative:

Step 1: The inboard and outboard span locations for the ailerons as fractions of the wing semispans were determined.

Step 2: The ratio between the chord length of the aileron and the chord length of the wing $\frac{c_{A}}{c}$ was determined, and the rolling moment effectiveness parameter $\frac{\beta c^{\prime}{ }_{l_{\delta}}}{k}$ was found from Figure 144, where $k$ is calculated using Equation 7.134.

$$
k=\left(c_{l_{\alpha}}\right)_{M} \frac{\beta}{2 \pi}
$$

where $\left(c_{l_{\alpha}}\right)_{M}$ is determined for the airfoil at the mean geometric chord of the portion of the wing with the aileron. 


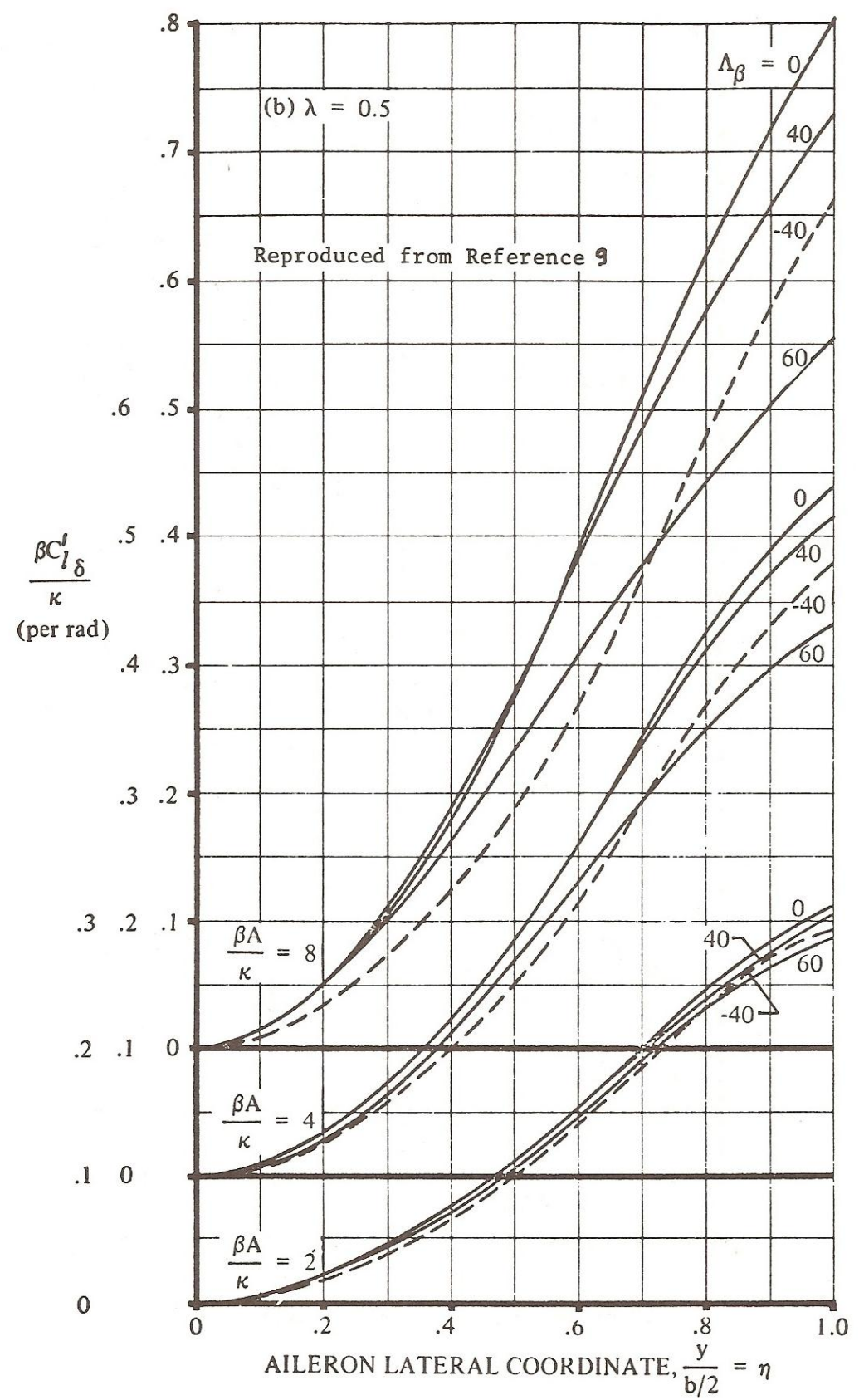

Figure 144: Effect of Symmetrical Flap Deflection on Yawing-Moment-due-to-Roll-Rate Derivative [Reproduced from Reference 42]

Equation 7.135 was used to calculate $\Lambda_{\beta}$, which is a necessary parameter to use with Figure 144 .

$$
\Lambda_{\beta}=\tan ^{-1}\left(\frac{\tan \Lambda_{c / 4}}{\beta}\right)
$$

Step 3: The rolling effectiveness of two full-chord controls anti-symmetrically deflected was determined using Equation 7.136. 


$$
c_{l_{\delta}}^{\prime}=\left(\frac{k}{\beta}\right)\left(\frac{\beta c_{l_{\delta}}^{\prime}}{k}\right)
$$

Step 4: The rolling effectiveness of partial-chord controls may be determined using Equation 7.137.

$$
c_{l_{\delta}}=\left(\alpha_{\delta_{A}}\right) c_{l_{\delta}}^{\prime}
$$

where:

$$
\begin{gathered}
\alpha_{\delta_{A}}=\frac{c_{l_{\delta}}}{\left(c_{l_{\alpha}}\right)_{A}} \\
c_{l_{\delta}}=\left\{\frac{c_{l_{\delta}}}{\left(c_{l_{\delta}}\right)_{\text {theory }}}\right\}\left(c_{l_{\delta}}\right)_{\text {theory }}
\end{gathered}
$$

$\frac{c_{l_{\delta}}}{\left(c_{l_{\delta}}\right)_{\text {theory }}}$ is found from Figure 145 and $\left(c_{l_{\delta}}\right)_{\text {theory }}$ is found from Figure 146.

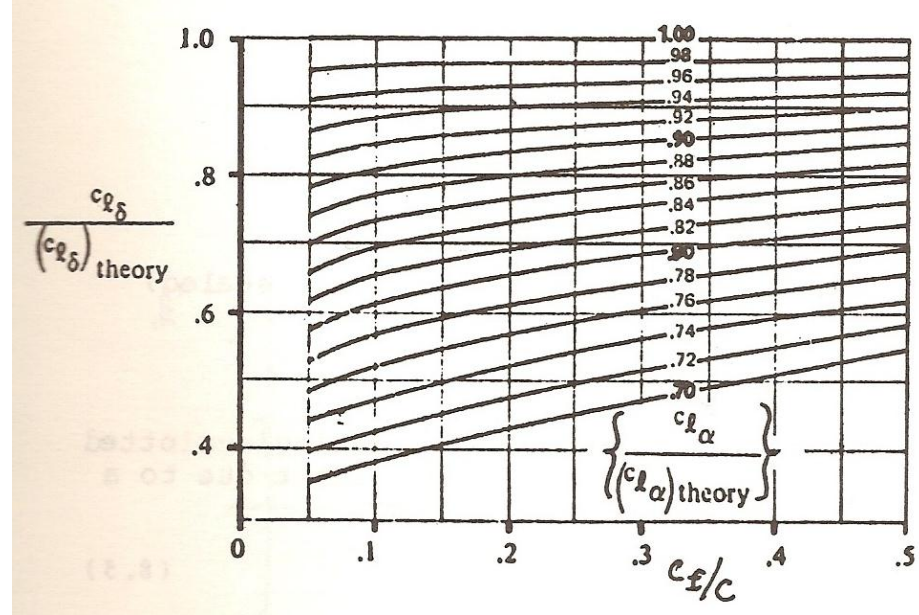

Figure 145: Correction Factor for Plain Flap Lift [Reproduced from Reference 42] 


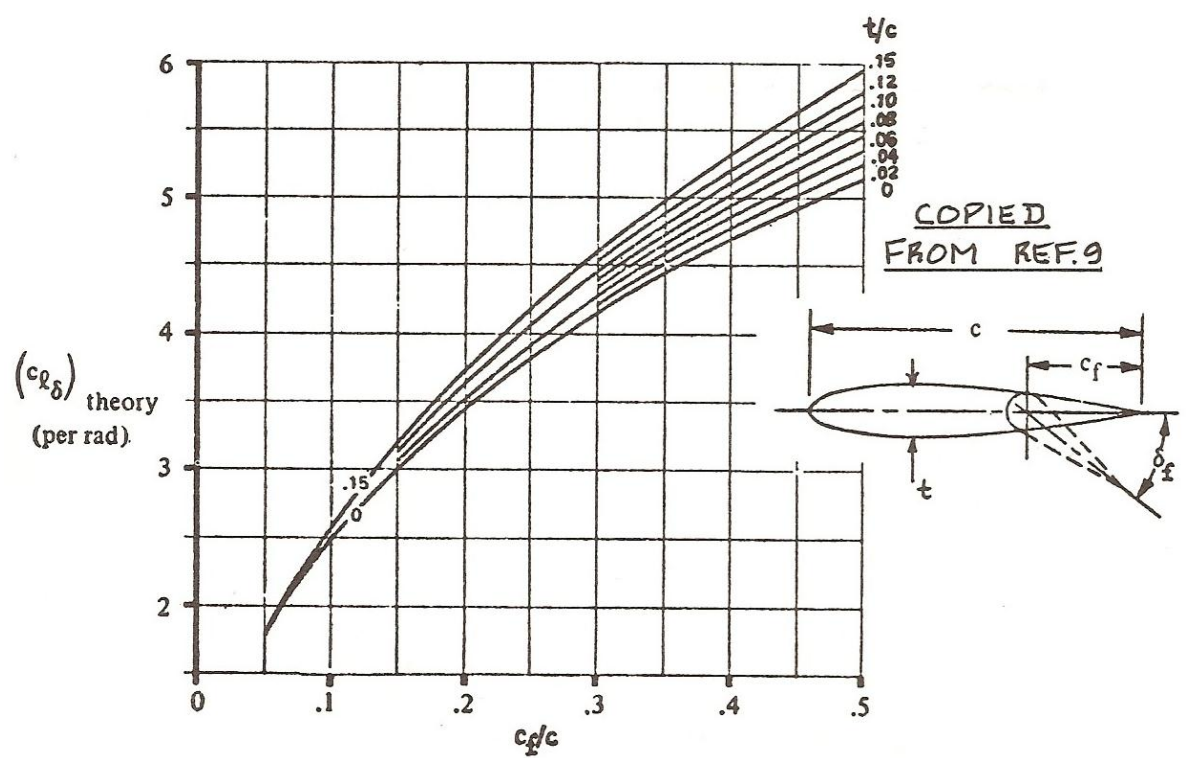

Figure 146: Lift Effectiveness of a Plain Flap [Reproduced from Reference 42]

Step 5: Equation 7.140 was used to account for the differential aileron control deflection effect to determine the total rolling moment coefficient for differential aileron control deflection. Special attention must be paid to the sign convention, where a positive deflection is considered a trailing edge down deflection.

$$
c_{l_{A}}=\left\{\left(\frac{c_{l_{\delta}}}{2}\right)_{\text {left }}+\left(\frac{c_{l_{\delta}}}{2}\right)_{\text {right }}\right\}\left(\delta_{A_{l e f t}}-\delta_{A_{\text {right }}}\right)
$$

Step 6: The total rolling-moment-due-to-aileron derivative is then calculated using Equation 7.141 .

$$
c_{l_{\delta A}}=\left(c_{l_{\delta}}\right)_{l e f t}+\left(c_{l_{\delta}}\right)_{\text {right }}
$$

\subsubsection{Rolling-Moment-Due-to-Rudder Derivative: $c_{l_{\delta R}}$}

The rolling-moment-due-to-rudder derivative was estimated using Equation 7.142, where $z_{v}$, $l_{v}$, and $\alpha$ are defined in Figure 120.

$$
c_{l_{\delta R}}=\left(\frac{z_{V} \cos \alpha-l_{V} \sin \alpha}{b}\right) c_{Y_{\delta R}}
$$

\subsubsection{Yawing-Moment-Due-to-Aileron Derivative: $c_{n_{\delta A}}$}

The yawing-moment-due-to-aileron derivative was estimated using Equation 7.143. 


$$
c_{n_{\delta A}}=K_{A} c_{L_{w}} c_{l_{\delta A}}
$$

where $K_{A}$ is the correlation constant for yawing moment due to aileron deflection found from Figure 147.
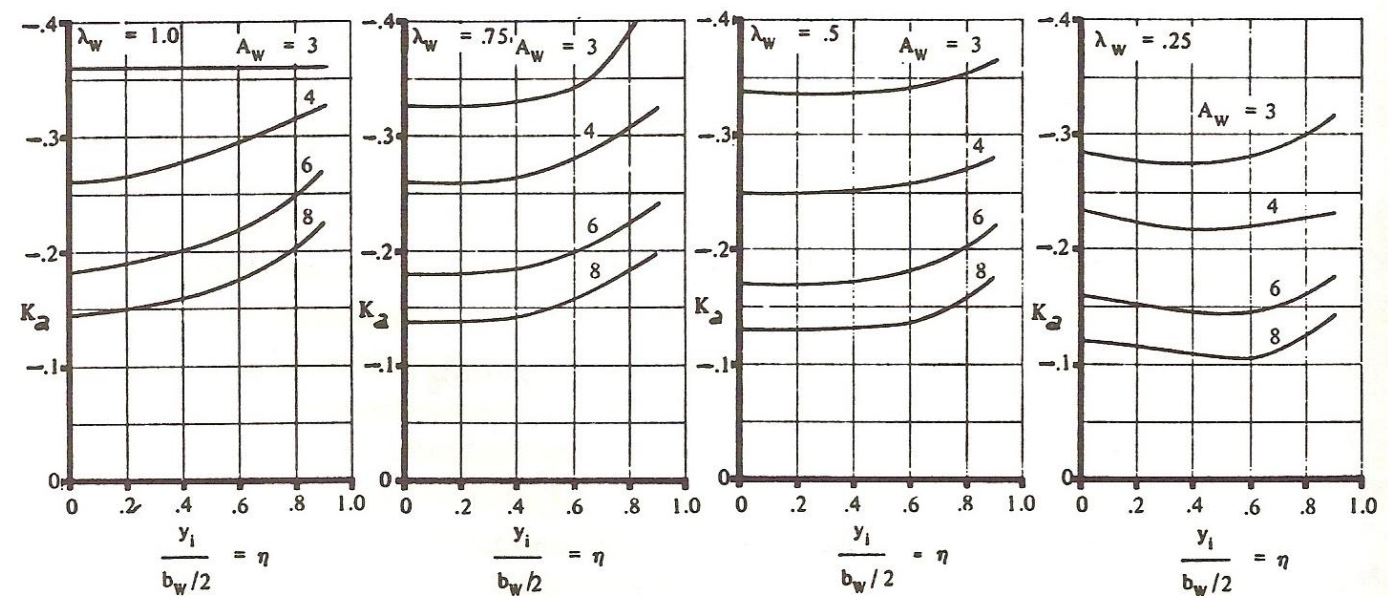

Figure 147: Correlation Constant for Yawing Moment due to Aileron Deflection [Reproduced from Reference 42]

For initial analysis, Equation 7.144 was used as for a preliminary estimation.

$$
c_{L_{w}}=c_{L_{1}}
$$

\subsubsection{Yawing-Moment-Due-to-Rudder Derivative: $c_{n_{\delta R}}$}

The yawing-moment-due-to-rudder derivative was estimated using Equation 4.227 where $z_{v}$, $l_{v}$, and $\alpha$ are defined in Figure 120.

$$
c_{n_{\delta R}}=-c_{Y_{\delta R}} \frac{\left(l_{V} \cos \alpha+z_{V} \sin \alpha\right)}{b}
$$

\subsubsection{PACT Landing Gear Drag Calculation}

The PACT aircraft has non-retractable (fixed) landing gears for both the main and nose gears. The landing gear drag coefficient for an aircraft with $i$ landing gears was computed from Equation 7.146.

$$
c_{D_{\text {gear }}}=S U M_{i}\left\lfloor\left(\left(c_{D_{\text {gear }_{L}=0}}\right)_{i}+p_{i} c_{L}\right) \frac{\left(S_{\text {gear }}\right)_{i}}{S}\right\rfloor
$$

where $c_{D_{\text {gear }} C_{L}=0}$ is the zero-lift drag coefficient of the landing gear based on its own reference area, $S_{\text {gear }}$, which was calculated using Equation 7.147, and $p$ is a factor to account for the variation of gear drag with lift. For the PACT aircraft, it is negligible $\approx 0$. 


$$
S_{\text {gear }}=b_{t} D_{t}
$$

Evaluating the landing gear wheels alone, the drag coefficient for a streamlined tire for the PACT was estimated to be $c_{D}=0.18$.

The main gears of the PACT aircraft were considered that of "Type 2" according to Reference 42. The drag coefficient for this style of landing gear without the wheel fairing was estimated as $c_{D_{\text {gaac }_{L}=0}}=0.565$ for entire main gear setup. The factor $p=0$ for this case, and Equation 7.147 were used to calculate the landing gear reference area.

Figure 148 shows the geometric parameters necessary to calculate the drag coefficient for the nose gear of the PACT aircraft. These geometric parameters were used to determine the nose gear drag incremental coefficient, which were used in the calculation of the total drag coefficient of the nose gear using Equation 7.146.

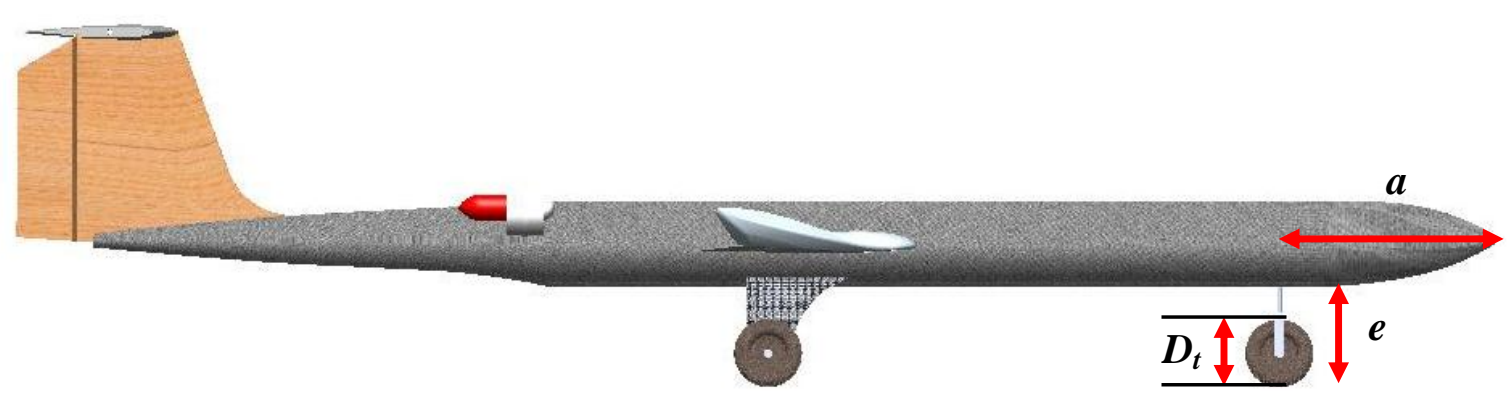

Figure 148: Nose Gear Geometric Parameters Necessary to Calculate the Drag Coefficient

The total drag coefficient for the landing gears was a sum of the drag coefficients for the main and nose gears for the PACT aircraft. 


\section{References}

1. Kebabjian, R. Statistics. Plane Crash Info Online. Accessed January 6, 2010. http://www.planecrashinfo.com/cause.htm.

2. National Transportation and Safety Board, "Aircraft Accident Report: USAir Flight 427," NTSB/AAR-99/01. US Government Printing Office, Washington, D.C. 20594. 1999.

3. National Transportation and Safety Board, "Aircraft Accident Report: United Airlines Flight 585,”NTSB/AAR-01/01. US Government Printing Office, Washington, D.C. 20594. 2001.

4. National Transportation and Safety Board, "Aircraft Accident Report: United Airlines Flight 232,” NTSB/AAR-90/06. US Government Printing Office, Washington, D.C. 20594 1990.

5. Byrne, Gerry. Flight 427: Anatomy of an Air Disaster. Copernicus Books. Springer-Verlag New York, Inc. 2002.

6. Perhinschi, M.G., Campa, G., Napolitano, M.R., Lando, M., Massotti, L., and Fravolini, M.L, "Modeling and Simulation of a Fault-Tolerant Flight Control System", International Journal of Modeling and Simulation, Vol. 26, No. 1, 2006.

7. Sghairi, M., de Bonneval, Y., Crouzet, J., Aubert, J., Brot, P. "Challenges in Building FaultTolerant Flight Control System for a Civil Aircraft.” IAENG International Journal for Computer Science. 35:4, IJCS_35_4_07.

8. Chen, J., Patton, R.J., Robust Model-Based Fault Diagnosis for Dynamic Systems, Kluwer Academic Publishers, Norwell, MA, 1999.

9. Bonivento, C., Isidori, A., Gentili, L., Marconi, L., Paoli, A., "Fault Detection and Isolation and Fault Tolerant Control." Research Publication supported by European Community under grant IST 2001-21122 IFATIS and MUIR. Accessed October 11, 2010. http://www.casy.deis.unibo.it/files/fdiftc.pdf.

10. Hwang, I., Balakrishnan, H., Tomlin, C., "Performance Analysis of Hybrid Estimation Algorithms", Proceedings of the $42^{\text {nd }}$ IEEE Conference on Decision and Control, vol. 5, December 2003.

11. Mihaylova, L., Semerdjiev, E., "An Interacting Multiple Model Algorithm for Stochastic Systems Control”, International Journal of Information \& Security, vol. 2, 1999.

12. Farmer, M.E., Hsu, R., Jain, A.K., "Interacting Multiple Model (IMM) Kalman Filters for Robust High Speed Human Motion Tracking”, Proceedings of the $16^{\text {th }}$ International Conference on Pattern Recognition. vol. 2, December 2002.

13. Rago, C., Prasanth, R., Mehra, R.K., Fortenbaugh, R., "Failure Detection and Identification and Fault Tolerant Control using the IMM-KF with applications to the Eagle-Eye UAV", Proceedings of the $37^{\text {th }}$ IEEE Conference on Decision and Control, Tampa, FL. December 1998.

14. Maciejowski, J.M., Jones, C.N., "MPC Fault-Tolerant Flight Control Case Study: Flight 1862", Control Group, Department of Engineering, University of Cambridge, Trumpington Street.

15. National Transportation and Safety Board, "Aircraft Accident Report: American Airlines Flight 96,” NTSB/AAR-73-2. US Government Printing Office, Washington, D.C. 20594. 1972.

16. Napolitano, M.R., Song, Y., Seanor, B. “On-line Parameter Estimation for Restructurable Flight Control Systems”, Elsevier Science Ltd. Aircraft Design 4 (2001) 19-50. 
17. Morelli, E.A. "Real-Time Parameter Estimation in the Frequency Domain," AIAA Atmospheric Flight Mechanics Conference. AIAA-99-4043, 1999.

18. Intelligent Flight Control: Advanced Concept Program - Annual Report. McDonnell Douglas Aerospace, St. Louis, Missouri. 1997.

19. Song, Y., Campa, G., Napolitano, M.R., Seanor, B., Perhinschi, M.G., "Comparison of Online Parameter Estimation Techniques within a Fault-Tolerant Flight Control System”, AIAA Journal of Guidance, Control, and Dynamics, 25(3), 2002, 528-537.

20. Perhinschi, M.G., Napolitano, M. R., Campa, G., Fravolini, M. L. "Integration of Fault Tolerant Systems for Sensor and Actuator Failures within the WVU NASA F-15 Simulator," AIAA Guidance, Navigation, and Control Conference 2003, August, 2003. Austin, TX.

21. Napolitano, M.R., Younghawn, A., Seanor, B., “A Fault-Tolerant Flight Control System for Sensor and Actuator Failures using Neural Networks", Aircraft Design, 3(2), 2000, 103 128.

22. Battipede, M., Gili, P., Napolitano, M.R., Perhinschi, M.G., Campa, G., "Performance in Different Failure Conditions of the Predictor-Corrector Neural Controller within the NASA IFCS F-15 WVU Simulator", Proceedings of the American Control Conference. Denver, Colorado, June 4-8, 2003.

23. Ward, D., Monaco, J., Barron, R., Bird, R., Virnig, J., Landers, T., "Self-Designing Controller: Design, Simulation, and Flight-Test Evaluation”, Final Tech. Report for Air Force Office of Scientific Research, Contract F49620-94-C-0087, Barron Associates, Ins., November 1996.

24. Ward, D., "Self-Designing Controller”, Final Report. Air Force Research Laboratory, AFRL WL-TR-97-3095, 1998.

25. Ward, D., Sharma, M., Richards, N.D., DeLuca, J., Mears, M., "Intelligent Control of Unmanned Air Vehicles: Program Summary and Representative Results", Air force Research Laboratory, AFRL-VA-WP-TP-2003-330. September 2003.

26. Monaco, J., Ward, D., Barron, R., Bird, R., "Implementation and Flight Test Assessment of an Adaptive, Reconfigurable Flight Control System", Proceedings of AIAA Guidance, Navigation, and Control Conference, New Orleans, Louisiana. August 1997.

27. Brinker, J.S., Wise, K.A., "Nonlinear Simulation Analysis of a Tailless Advanced Fighter Aircraft Reconfigurable Flight Control Law", Proceedings of the AIAA Guidance, Navigation, and Control Conference, Portland, Oregon. August 9-11, 1999. AIAA-994040.

28. Brinker, J.S., "Reconfigurable Flight Control for a Tailless Advanced Fighter Aircraft", Proceedings of AIAA Guidance, Navigation, and Control Conference, Boston, Massachusetts, August 10-12, 1998. AIAA-98-4107.

29. Calise, A.J., Lee, S., Sharma, M., "Direct Adaptive Reconfigurable Control of a Tailless Advanced Fighter Aircraft", Proceedings of the AIAA Guidance, Navigation and Control Conference, Boston, Massachusetts, August 10-12, 1998. AIAA-98-4108.

30. Wise, K.A., Brinker, J.S., Calise, A.J., Enns, D., Elgersma, M., Voulgaris, P., "Direct Adaptive Reconfigurable Flight Control for a Tailless Advanced Fighter Aircraft", International Journal of Robust Nonlinear Control, Special Issue on Reconfigurable Flight Contrl, 9, 999-1012. 1999.

31. Kim, B.S., Calise, A.J., "Nonlinear Flight Control Using Neural Networks", Journal of Guidance, Control and Dynamics, vol. 52, 26-33. 1997. 
32. Wise, K.A., "Reconfigurable Systems for Tailless Fighter Aircraft - RESTORE”, Final Report, AFRL-VA-WP-TR-99-3067.

33. Eberhardt, R., Niestroy, M., Tallant, G., Monaco, J., Ward, D., "Reconfigurable Systems for Tailless Fighter Aircraft", Lockheed Martin Tactical Aircraft Systems and Barron Associates, Inc. Final Technical Report for Air Force Research Laboratory, AFRL-VAWP-TR-1999-3078, November 1999.

34. Brinker, J.S., Wise, K.A., "Flight Testing of a Reconfigurable Flight Control Law on the X36 Tailless Fighter Aircraft," Proceedings of the AIAA Guidance, Navigation, and Controls Conference, Denver, Colorado. August 2000. AIAA-2000-3941.

35. Brinker, J.S., Wise, K.A., "Flight Testing of Reconfigurable Flight Control Law on the X-36 Tailless Aircraft," Journal of Guidance, Control and Dynamics, vol. 24, issue 5, 903 909., 2001.

36. Vijayakumar, J., Schmitz, D., Balakrishnana, S.N., "Development and Implementation of New Nonlinear Control Concepts for a UA", IEEE 0-7803-8539-x/04. 2004.

37. Christmann, H.C., Christophersen, H. B., Wu, A.D., Johnson, E.N., Guidance, Navigation, Control, and Operator Interfaces for Small Rapid Response Unmanned Helicopters. Georgia Institute of Technology, Atlanta, GA. 2008.

38. Bethke, B., How, J.P., Vian, J., "Group Health Management of UAV Teams with Applications to Persistent Surveillance”, American Control Conference, Seattle, Washington. June 11-13, 2008.

39. Teo, R., Jang, J.S., Tomlin, C.J., "Automated Multiple UAV Flight - the Stanford DragonFly UAV Program", $43^{\text {rd }}$ IEEE Conference on Decision and Control. Atlantis, Paradise Island, Bahamas. December 14-17, 2004.

40. Murch, A.M., A Flight Control System Architecture for the NASA AirSTAR Flight Test Infrastructure. NASA Langley Research Center, Hampton, VA.

41. Hoak, D.E, et al. "The USAF Stability and Control DATCOM", Air Force Wright Aeronautical Laboratories, TR-83-2048, October 1960 (revised in 1978).

42. Roskam J. “Airplane Design. Part I - Part VIII”, Roskam Aviation and Engineering Corporation, Lawrence, Kansas, 1990.

43. Advanced Aircraft Analysis (AAA), Design, Analysis, and Research (DAR) Corporation, 120 East Ninth Street, Suite 2, KS, 66064.

44. SimGen. Software Package, Ver. 1.0, Bihrle Applied Research Corporation, 81 Research Drive, Hampton, VA, 23666.

45. Jodeh, N.M., "Development of Autonomous Unmanned Aerial Vehicle Research Platform: Modeling, Simulating, and Flight Testing", Master of Science, Thesis. Department of Aeronautics and Astronautics, Air Force Institute of Technology. March 2006.

46. Jodeh, N.M., Blue, P.A., "Development of Small Unmanned Aerial Vechile Research Platform:Modeling and Simulating with Flight Test Validation", AIAA Modeling and Simulation Technologies Conference and Exhibit. Keystone, CO. August 21-24, 2006. AIAA 2006-6261.

47. Jung, D., Tsiotras, P., "Modeling and Hardware-in-the-Loop Simulation for a Small Unmanned Aerial Vehicle", Infotech@Aerospace 2007 Conference and Exhibit. Rohnert Park, CA. May 7-10, 2010. AIAA 2007-2768.

48. Orr, M.W., Rasmussen, S.J., Karni, E.D., Blake, W.B., "Framework for Developing and Evaluating MAV Algorithms in a Realistic Urban Setting”, 2005 American Control Conference. Portland, OR. June 8-10, 2005. FrB04.3. 
49. Blake, W.B., "Missile DATCOM Users Manual,” AFRL Technical Report AFRL-VA-WPTR-1998-3009, 1998.

50. Foster, T.M., "Dynamic Stability and Handling Quatlities of Small Unmanned-AerialVehicles", Master of Science, Thesis. Department of Mechanical Engineering, Brigham Young University. April 2005.

51. Klein, Vladislav and Morelli, Eugene A., Aircraft System Identification: Theory and Practice. AIAA Education Series, American Institute of Aeronautics and Astronautics, Inc. Reston, VA. 2006.

52. Morelli, E.A. “In-Flight System Identification,” AIAA-98-4261, 1998.

53. Maine, R.E., Iliff, K.W., “Application of Parameter Estimation to Aircraft Stability and Control: The Output-Error Approach", NASA Reference Publication 1168, June 1986.

54. Maine, R. E., Iliff, K. W., "Identification of Dynamic Systems: Theory and Formulation", NASA RF 1168, June 1986.

55. Mendel, J. M., Discrete Techniques of Parameter Estimation, Marcel Dekker, New York, 1973.

56. Iliff, K. W., Maine, R. E., "Practical Aspects of Using a Maximum Likelihood Estimation Method to Extract Stability and Control Derivatives from Flight Data", NASA TN D8209, 1976.

57. Iliff, K. W., Taylor, L. W., "Determination of Stability Derivatives from Flight Data Using a Newton-Raphson Minimization Technique", NASA TN D-6579, 1972.

58. Wang, K. C.; Iliff, K. W., 2006, "Retrospective and Recent Examples of Aircraft Parameter Identification at NASA Dryden Flight Research Center", Journal of Aircraft, Vol. 41, No. 4, pp. 752-764.

59. Iliff, K.W., Wang, K.C., "Extraction of Lateral-Directional Stability and Control Derivatives for the Basic F-18 Aircraft at High Angles of Attack," NASA Technical Memorandum 4786, February 1997.

60. Iorio, C., Napolitano, M.R., Seanor, B., Younghwan, A., Bowers, A.H., "Parameter Estimation for the NASA SR-71 Longitudinal and Lateral-Directional Dynamics," Proceedings of AIAA Atmospheric Flight Mechanics Conference, Portland, Oregon, August 9-11, 1999. AIAA-1999-4172.

61. Seanor, B., Song, Y., Napolitano, M.R., Campa, G., "Comparison of On-Line and Off-Line Parameter Estimation Techniques using NASA F/A-18 HARV Flight Data," Proceedings of AIAA Atmospheric Flight Mechanics Conference, Montreal, Canada, August 6-9, 2001. AIAA-2001-4261.

62. Yongkyu Song, Marcello Napolitano, "A Comparative Study of Real-Time Aircraft Parameter Identification Schemes Applied to NASA F/A-18 HARV Flight Data", Trans. Japan Soc. Aero. S Sci., Vol. 45 (2002), pp.180-188.

63. Napolitano, M. R., Paris, A., Seanor, B., Bowers, A. H., "Estimation of the LateralDirectional Aerodynamic Parameters from Flight Data for the NASA F/A-18 HARV", Proceedings of the AIAA Atmospheric Flight Mechanics 96 Conference, San Diego, AIAA Paper 96-3420, 1996.

64. Perhinschi, M.G., Napolitano, M.R., "Flight Data Reduction Methodology for Performance Evbaluation and Comparison of Model-Following Adaptive Control Laws," The Aeronautical Journal, Paper No. 3221, pg. 807-814. December 2007. 
65. Jategaonkar, Ravindra V., Flight Vehicle System Identification: A Time Domain Methodology. American Institute of Aeronautics and Astronautics, Inc. Reston, VA. 2006.

66. Ljung, Lennart, System Identification: Theory for the User. $2^{\text {nd }}$ Edition. Prentice Hall PTR, Upper Saddle River, NJ. 1999.

67. ZAERO Software Package. Zona Technology, Inc. Accessed March 28, 2010. http://www.zonatech.com/ZAEROInformation.htm.

68. Napolitano, M.R. "Development of Formation Flight Control Algorithms Using 3 YF-22 Flying Models," AFOSR Final Report, AFOSR Grant F49620-01-1-0373, April 2005.

69. Campa, G., Gu, Y., Seanor, B., Napolitano, M.R., Pollini, L., and Fravolini, M.L., "Design and Flight Testing of Non-Linear Formation Control Laws", Control Practice Engineering: A Journal of the International Federation of Automatic Control, 15 (2007), 1077-1092.

70. Phillips, K., Campa, G., Gururajan, S., Seanor, B., Napolitano, M.R., Gu, Y., Fravolini, M.L., "Nonlinear Aircraft Model Identification and Validation for a Fault-Tolerant Flight Control System", AIAA 2010 Atmospheric Flight Mechanics Conference, Toronto, Ontario Canada. August 2-5, 2010.

71. Campa, G. "Airlib, The Aircraft Library", 2003. http://www.mathworks.com/matlabcentral/

72. Napolitano, M.R., Paris, A., Seanor, B., Bowers, A.H. "Estimation of the Longitudinal Aerodynamic Parameters from Flight Data for the NASA F/A-18 HARV", AIAA Paper 96-3419, Proceedings of the AIAA Atmospheric Flight Mechanics Conference 1996, San Diego, CA, July 1996.

73. Bowers, A.H., Pahle, J.W., Wilson, R.J., Flick, B.C., Rood, R.L. “An Overview of the NASA F-18 High Alpha Research Vehicle,” NASA Technical Memorandum 4772. Dryden Flight Research Center, Edwards, CA. October 1996.

74. Simulink, Simulation, and Model Based Design, The Mathworks Inc., Natick, MA, 2007.

75. Flint, T.W. \& Vaccaro, R.J., "Performance Analysis of N4SID State-Space System Identification," Proceedings from the American Control Conference. Philadelphia, PA. June 1998.

76. Van Overschee, P. \& De Moor, B., "N4SID: Subspace Algorithms for the Identification of Combined Deterministic-Stochastic Systems," Pergamon Press, Ltd. Automatica Vol. 30, No. 1, pg. 75-93, 1994.

77. Xie, X. and Lu, C., "Optimization and Coordination of Wide-Area Damping Controls for Enhancing the Transfer of Capability of Interconnected Power Systems," Elsevier, Science Direct Online. Electric Power Systems Research 78 (2008) 1099-1108. http://www.sciencedirect.com/

78. Lee, J.H., Choi, J.H., Lee, K.S., "Identification Methods," 1997.

79. Smith, M.S., Moes, T.R., Morelli, E.A., "Real-Time Stability and Control Derivative Extraction from F-15 Flight Data," National Aeronautics and Space Administration, Dyrden Flight Research Center, Edwards, CA. NASA/TM-2003-212027. September 2003.

80. Perhinschi, M.G., Lando, M., Massotti, L., Campa, G., Napolitano, M.R., Fravolini, M.L. "Real- Time Parameter Estimation Issues for the NASA IFCS F-15 Fault-Tolerant Systems," AIAA American Control Conference, 2002. ACC02-AIAA1079. 
81. Campa, G., Gu, Y. “F-22 Linear Identification,” WVU Technical Report, Department of Mechanical and Aerospace Engineering, College of Engineering and Mineral Resources, West Virginia University, Morgantown, WV. February 2002.

82. Campa, G. "PIL, Parameter Identification Library", 2008. http://www.mathworks.com/matlabcentral/

83. Etkin, B., Dynamics of Atmospheric Flight. John Wiley \& Sons, Inc. 1972.

84. Rauw, M.O., "FDC 1.2 - A Simulink Toolbox for Flight Dynamics and Control Analysis." Zeist, The Netherlands, 1997. ISBN:90-807177-1-1, http://www.dutchroll.com

85. Stevens, B. and Lewis, F. Aircraft Control and Simulation. $2^{\text {nd }}$ Ed. John Wiley \& Sons, Inc. Hoboken, NJ. 2003.

86. Brumbaugh, R.W. "An Aircrat Model for the AIAA Controls Design Challenge," NASA Contractor Report 186019, December 1991.

87. Roskam, J. Airplane Flight Dynamics and Automatic Flight Controls - Part I, Design, Analysis, and Research Corporation, Lawrence, KS. 2003.

88. Maine, R.E., Iliff, K.W., The Theory and Practice of Estimating the Accuracy of Dynamic Flight-Determined Coefficients, NASA Reference Publication 1077. July 1981.

89. Sorenson, H.W., Parameter Estimation, Marcel Dekker, Inc., New York, 1980.

90. Moore, D.S., McCabe, G.P., Introduction to the Practice of Statistics, $4^{\text {th }}$ ed. Purdue University. W.H. Freeman and Company, New York, 2003.

91. Abbott, I.H., Von Doenhoff, A.E., Theory of Wing Sections, Dover Publications, Inc. New York, NY. 1959.

92. Gross, J., Gu, Y., Seanor, B., Gururajan, S., Napolitano, M.R. Advanced Research Integrated Avionic (ARIA) System for Fault-Tolerant Flight Research, AIAA Guidance, Navigation, and Control Conference 2009. Chicago, IL. August 10-13, 2009. 